Portland State University

PDXScholar

Fall 11-21-2014

\title{
Confined Mesoscopic Fluid-like Films Analyzed with Frequency Modulation and Acoustic Detection
}

Rodolfo Fernandez Rodriguez

Portland State University

Follow this and additional works at: https://pdxscholar.library.pdx.edu/open_access_etds

Part of the Other Physics Commons

Let us know how access to this document benefits you.

\section{Recommended Citation}

Fernandez Rodriguez, Rodolfo, "Confined Mesoscopic Fluid-like Films Analyzed with Frequency Modulation and Acoustic Detection" (2014). Dissertations and Theses. Paper 2047.

https://doi.org/10.15760/etd.2046

This Dissertation is brought to you for free and open access. It has been accepted for inclusion in Dissertations and Theses by an authorized administrator of PDXScholar. Please contact us if we can make this document more accessible: pdxscholar@pdx.edu. 
Confined Mesoscopic Fluid-like Films Analyzed with Frequency

Modulation and Acoustic Detection

by

Rodolfo Fernández Rodríguez

A dissertation submitted in partial fulfillment of the requirements for the degree of

Doctor of Philosophy

in

Applied Physics

\author{
Dissertation Committee: \\ Andres La Rosa, Chair \\ John Freeouf \\ Erik Sanchez \\ Raj Solanki \\ Shankar Rananavare \\ Bin Jiang
}

Portland State University

2014 
(C) 2014 Rodolfo Fernández Rodríguez 


\begin{abstract}
Complete understanding of the physics underlying the changes in viscoelasticity, relaxation time, and phase transitions that mesoscopic fluid-like systems undergo at solid-liquid interfaces or under confinement remains one of the major challenges in condensed matter physics. Moreover, studies of confined mesoscopic fluid films are relevant to technological areas like adhesion, wetting processes and nanotribology.

This thesis addresses the interaction between two sliding solids interfaces separated by a nanometer sized gap, with emphasis on the role of the mesoscopic fluid film trapped between them. For this purpose we integrated two acoustic techniques, recently introduced by our group, into a sub-nanometer precision and thermal drift corrected scanning probe microscope (SPM): the shear-force/acoustic near-field Microscope (SANM) and the whispering gallery acoustic sensing (WGAS). The SANM monitors the sound waves originating in the probe-layer interaction while the motion of the probe is monitored by the WGAS. Additionally, we decouple the interaction forces by using frequency modulation and measure the local tunneling current to help establish the location of the substrate. Our results show a strong correlation between the elastic component of the probe's interaction and the SANM amplitude, as well as between the phase lag response of the fluid relative to the probe's excitation (represented by the SANM phase) and the onset of the probe-sample contact region. Frequency modulation SANM-WGAS brings a new acoustic sensing mechanism to the challenging characterization of fluid-like physical systems at the nanometer scale.
\end{abstract}




\section{DEDICATION}

I dedicate this dissertation to my family. Rodolfo, Elena, Carolina, Carlos. 


\section{ACKNOWLEDGEMENTS}

I cannot express enough gratitude to my committee for their constant support and encouragement: Dr. Andres La Rosa, my committee chair; Dr. John Freeouf, Dr. Erik Sanchez, Dr. Raj Solanki, Dr. Shankar Rananavare and Dr. Bin Jiang.

My thanks to Marc, Alex Ch. and Theo without whose help the completion of this work could not have been accomplished. Friends, new and old: Mike 1, Mike 2, Micah, Justin, A.J., Lester. Teaching me what friendship is made off.

Thanks to my siblings. Nobody can supplant your mix of support and sarcasm.

Finally, my parents. Your encouragement and support meant a heartening breeze when everything was grey and gloomy. In the end, all is as it should be. 


\section{TABLE OF CONTENTS}

ABSTRACT i

DEDICATION ii

ACKNOWLEDGEMENTS iii

LIST OF FIGURES viii

LIST OF ACRONYMS xix

CHAPTER 1 SCIENCE and TECHNOLOGY ISSUES UNDERLYING THIS THESIS 1

1.1 The unique physical properties of mesoscopic fluid films_ 1

1.2 The relevance of gaining a better understanding of mesoscopic film properties 3

1.3 Context: the emphasis on the study of interfaces 7

1.4 Standard surface characterization techniques 9

1.5 Shear-force acoustic near-field microscopy (SANM) 11

CHAPTER 2. NEAR FIELD ACOUSTIC MICROSCOPY in the CONTEXT of SCANNING PROBE MICROSCOPY DEVELOPMENTS 14

2.1 Scanning probe microscopy___ 15

2.2 Quartz tuning fork scanning probe microscopy____ 16

$2.3 \quad$ Whispering gallery acoustic sensing___ 21

2.4 Shear-force acoustic near-field microscopy (SANM)___ 23

2.5 Frequency modulation in scanning probe microscopy__ 25

2.6 Equations for the calculation of interaction forces__ 28

CHAPTER 3. INTEGRATION Of SHEAR-FORCE ACOUSTIC NEAR-FIELD MICROSCOPY (SANM) and WHISPERING GALLERY ACOUSTIC SENSING (WGAS) INTO QTF-SPM 35

3.1 Experimental setup 36 
3.2 Components overview 38

3.2.A The OC-4 PLL Nanonis system___ 39

3.2.B The FPGA card ___ 40

3.2.C The FPGA breakout box___ 42

3.2.D The high resolution stage (HRS)__ 42

3.2.E The pre-amplifiers___ 44

3.2.F Custom made cables for mechanical decoupling__ 45

3.2.G Piezo-acoustic sensors__ 46

3.2.H Nano-Z50HS_ 46

3.3 Signal conditioning___ 46

3.3.A Trans-impedance amplifier___ 47

3.3.B Active filtering__ 49

3.4 Software development in LabVIEW _ 50

3.4.A Modified proportional integral differential (PID) feedback for high resolution control__ 52

3.4.B Approach and retraction control program (ACRP)_ 52

3.5 Determination of the amplitude of oscillation of the QTF_ 56

3.6 Tunneling current measurements___ 62

3.7 Probe fabrication and inspection 65

CHAPTER 4. CALIBRATION and DRIFT CORRECTION___ 69

4.1 Calibration of PZT with feedback mode___ 71

4.2 Approaching and retracting curves operation___ 83

4.3 Drift compensation mode___ 85

CHAPTER 5. FREQUENCY MODULATION in the WGAS-SANM QTF-SPM___ 87

5.1 Frequency modulation___ 88 
5.2 Frequency shift as control signal

5.3 Acoustic sensor to determine the real amplitude variation_96

CHAPTER 6. CHARACTERIZATION Of MESOSCOPIC FILMS UNDER CONFINEMENT.

The CASE of GRAPHITE, MICA and SILICON SURFACES 98

6.1 Highly oriented pyrolytic graphite (HOPG)__ 100

6.1.A Sample preparation 100

6.1.B Probing in the low interaction forces regime (up to $5 \mathrm{nN}$ ) 101

6.1.C Case: first approaches 102

6.1.D Case: subsequent approaches 109

6.1.E Analysis of subsequent approaches 112

6.1.F Viscous and elastic behavior 116

6.1.G Discussion about the phase of the SANM 118

6.1.H Discussion about the SANM amplitude and the elastic shear force 120

6.1.I Section conclusions 122

6.2 Mica 123

6.2.A Sample preparation 124

6.2.B Initial approaches 125

6.2.C Subsequent approaches 128

6.2.D Analysis of subsequent approaching and retracting curves (ARCs) 129

6.2.E Section conclusions 133

6.3 Hydrogen terminated silicon 134

6.3.A Sample preparation 134 
6.3.B Stable approaches

CHAPTER 7. CONCLUSIONS 141

7.1 Future outlook 143

BIBLIOGRAPHY 144

APPENDIX A. Calculation of the elastic and dissipation interaction forces 156

APPENDIX B. Calculation of the piezo-electro-mechanical coupling $\alpha$ 159

APPENDIX C. The problem of the changing tunneling impedance 161

APPENDIX D. Difference in the force analysis between a QTF and a cantilever type probes 163 


\section{LIST OF FIGURES}

Figure 1. Energy dissipation in interfacial friction phenomena by the phononic mechanism. As the solid substrate oscillates harmonically, it produces deformation on the liquid layer; anharmonic motion responses in the liquid (i.e. out of synchronization with the substrate motion) lead to a net dissipation of energy[36].

Figure 2. Monitoring the acoustic response from confined mesoscopic fluid trapped between solid surfaces in relative motion. Left: The apex of a laterally-oscillating probe is brought into shear interaction with an adsorbed mesoscopic fluid film, engendering an acoustic wave that couples to the substrate, travels through the substrate bulk, and is finally detected by an acoustic transducer. Right: A metallic probe and metallic sample monitors the onset of current (blue trace). Notice the detection of acoustic SANM-signal even when there is not tunneling current. 12

Figure 3. Atomic Force Microscope experimental setting diagram. 16

Figure 4. RCL circuit equivalent to the mechanical Butterworth-K. S. Van Dyke QTF model.

Figure 5. Quartz Tuning Fork Scanning Probe Microscopy (QTF-SPM). 20

Figure 6. Whispering Gallery Acoustic Sensing (WGAS) implementation for monitoring the mechanical lateral oscillations of the electrically driven $32 \mathrm{kHz}$ quartz tuning fork to which a probe is firmly attached. The amplitude of the oscillatory motion of the tines of the QTF sets an acoustic wave that travels up to the cavity where a series of standing acoustic waves are established. An acoustic transducer is judiciously located at the periphery of the cavity to maximize the detected signal.

Figure 7. SANM experimental setup. The lateral oscillations of the probe, upon its interaction with the adsorbed fluid layer, engenders an acoustic wave, which is detected by the acoustic transducer located at the bottom in mechanical contact with the sample.

Figure 8. Effects of conservative and dissipative forces on the spectrum of a QTF. 27 
Figure 9. Diagram of the WGAS and SANM integration into a QTF-SPM. The monitoring of the tunneling current is optional (when testing conductive samples); in that case the ac-voltage driving the QTF is used to bias the metallic probe. 37 Figure 10. Front panel of the OC-4 PLL stage. The outputs to the right are not shown. 40 Figure 11. FPGA card from National Instruments (NI). http://www.ni.com/fpga/ 41 Figure 12. Front panel of the FPGA breakout box. The connectors shown (apart from input, output fine and output coarse) can be exchanged internally using FPGA programming. 43

Figure 13. High resolution stage (HRS) front panel. 44

Figure 14. Gain of the preamplifier of the SANM sensor. The gain at $32 \mathrm{kHz}$ (indicated by the circle) is $3950 \mathrm{~V} / \mathrm{A}$. 45

Figure 15. Schematic of the noise addition. Each stage introduces noise to final signal output 46

Figure 16. Top: Trans-impedance amplifier circuit diagram. Bottom: Board implementation.

Figure 17. Left: Diagram of the QTF and trans-impedance amplifier. Right: Actual transimpedance amplifier circuit inside its enclosure displaying its assembled surface mount components.

Figure 18. Gain response of the QTF/WGAS-preamplifier (left) and its corresponding noise spectral density (right). 49

Figure 19. Active low pass filter of Sallen-Key topology. This design only needs one operational amplifier per filter at the expense of smaller roll off[98]. 50 Figure 20. High resolution output (HRO). Two 16 bit signals are synchronized to produce a continuous higher resolution (100 times higher in this thesis) than each channel could deliver separately. The resulting output value is vout $=v 1 \times R / R+v 2 \times \beta \times R / R=$ $\mathrm{v} 1+\mathrm{v} 2 \times \beta$. 53

Figure 21. Approaching and Retracting Curve Program (ARCP) developed to control and perform drift corrections on every approaching and retracting curve (ARC). 55 
Figure 22. Raw data of the QTF spectrum. The QTF was kept in its vacuum metal container and soldered right next to the input of the board of the pre-amplifier. One of the tines of the QTF is grounded and the other connected to the input of the preamplifier. 56

Figure 23. Noise density spectrum of the QTF obtained by calculating the standard deviation of the average signal from the spectrum in Figure 22 above. The algorithm used had an averaging window of 100 points equivalent to a $1.5 \mathrm{~Hz}$ range. 57 Figure 24. Schematic connections to measure the QTF's response due to thermal noise.

Figure 25. Thermal noise spectrum and fitting curve of a QTF still enclosed in its metal cylinder but connected to the amplifier with a $20 \mathrm{~cm}$ of coaxial cable. The capacitance of the connections increase the asymmetry of the spectrum. 58 Figure 26. Spectrum taken to an actual QTF right before the approach and retraction process, hovering around $10 \mu \mathrm{m}$ from the sample. The excitation voltage is $1 \mathrm{mV}$. The values obtained are $\mathrm{C}=2.83 \times 10^{-15}$ and $\alpha=4.69 \times 10^{-6} \mathrm{C} / \mathrm{m}$. 60 Figure 27. The amplitude and phase response of the WGAS signal. The red vertical line marks the resonance frequency. 62 Figure 28. Graph from Freund et al. [105] showing the dependence of tunneling current and distance from the sample with low relative humidity $(\mathrm{RH})$. There is no indication of sharp onset of tunneling current due to the water layer. Right, same process in high $\mathrm{RH}$ levels. There is the indication of the sudden jump in tunneling current when the probe enters the adsorbed water layer. 63 Figure 29. Graph from Opitz et al. [106] showing the behavior of a typical tunneling current approach curve at ambient conditions. 63

Figure 30. Monitor screen of the Field Emission Scanning Electron Microscope (FE-SEM) Hitachi S-4160 restored in our laboratory. 66 
Figure 31. SEM image of the AFM calibration grid used for calibration of the images. The edges of the square were determined by taking the maximum peak in the case of a bright edge, and halfway the slope for a dim edge.

Figure 32. FE-SEM images of a test probe before the experiment. Right: The probe protrudes approximately $70 \mu \mathrm{m}$ from the edge of the tine of the QTF. 68

Figure 33. Experimental setup for the PZT calibration procedure. 73

Figure 34. Schematic representation of the PZT calibration process. When the probe is in feedback, the PZT tracks the motion of the surface, which rests on a comercial piezostage with closed loop positioning. 73

Figure 35. Raw data of the PZT driving voltage in the calibration measurement process. The beginning and ending values of the PZT driving voltage do not coincide. As expected the system has drifted. Since the MCL piezostage determines its position with closed loop control the cause of the discrepancy must be thermal drift of the stage where the PZT is mounted on. 74

Figure 36 . Hysteresis curve after the drift velocity correction. 75

Figure 37. Resulting curves obtained from substracting the fitted linear curve from the experimental hysteresis curve. The average of both curves is also shown. 76 Figure 38. Fitted curve of the average PZT hysteresis. 77 Figure 39. Top: Frequency shift as control signal for feedback control. The calibration driving signal of the piezotube is shown below. The probe is in feedback after second 25 and it follows the drift of the surface. 78

Figure 40. Same positioning curve showing the moment when the probe enters in contact with the surface. The effect of creep, while in feedback, is expected to bring the probe closer to the sample showing a decrease in the control signal (in an attempt to pull away from the surface) but this behavior is not observed. The slow downward change is attributed to thermal drift. 79 Figure 41. Same PZT positioning curve from Figure 39 and Figure 40. The voltage variation (peak to peak uncertainty in the position) is roughly $1 \AA$. 80 
Figure 42. Signal from the strain gauge, showing the change in the position of the $\mathrm{MCL}$ piezo-stage while in feedback.

Figure 43. Driving signal of the PZT, while in feedback. The curves show that there is a delay of $122 \mathrm{~ms}$ for a velocity of $4 \mathrm{~nm} / \mathrm{s}$. The actual experiments were performed with a $2 \mathrm{~nm} / \mathrm{s}$ velocity approach which would amount to about $61 \mathrm{~ms}$ between the actual changes in the position.

Figure 44. Signal of the feedback error signal for the calibration process. The graph shows the time response of the frequency shift signal. It is around 100 ms, which was intentionally tuned to have the greatest precision at the cost of time response. This means that only approximately $12 \mathrm{~ms}$ correspond to the PZT response time.

Figure 45. The process of approaching and retracting curves (ARC). After the air-fluid interface is found, (approaching the surface with $5 \mathrm{~nm} / \mathrm{s}$ speed), a fast retraction follows and then the actual ARC is performed. When the acquisition is finished the tip is retracted and the cycle starts again.

Figure 46. Procedure to estimate the value of the drift. After finding the surface and retracting (initial position) the feedback is turned on and the tip will approach the sample, stay in the predetermined feedback distance and the average value is recorded. Then the tip is moved to a known constant position away from the surface. Finally the tip is moved back into feedback and the position recorded again. If there were no drift, both initial and final positions would be equal, but because of the drift the difference is different than zero. Since the time between the two events is known, the calculation of the drift velocity is straightforward. 86 Figure 47. Schematic of an amplitude modulated SPM. There is only one feedback loop (thick arrows), which is used to control the probe-sample distance. PID stands for proportional-integral-differential, although in SPM application the differential gain is set to zero resulting in only PI control. 88 Figure 48. The new feedback loop for FM-SPM includes a feedback inner loop (double line arrows) to keep the phase constant at 90 degrees. 89 
Figure 49. The signal demodulating block. It is physically located inside the OC-4 system. An additional external pre-amplifier was included between the QTF and the SC \& DM input (section 3.2.1). 90

Figure 50. Signal generating block (voltage control oscillator). It drives the frequency changes when the system is in frequency modulation (FM) mode. 91 Figure 51. Simple PID block receiving control parameter for the probe-sample distance feedback. 92

Figure 52. Block diagram of the SPM amplitude modulation (AM-SPM). Notice a single PID loop, the QTF probe-sample distance control. 92

Figure 53. The dashed line shows the addition of an inner PID loop that takes care of the QTF excitation. As opposed to AM scheme the FM scheme actively controls the QTF's amplitude, phase and frequency.

Figure 54. Drift experimental evaluation using FM mode with the frequency shift as the control signal. The two upper lines (blue) are the drift slope while in feedback and the lower line (red) is the average calculated by the ARCP. 95

Figure 55. Step motion inside the interaction region showing the difference in time response between FM and AM. 95

Figure 56. Time response of the frequency shift used in the approaching and retracting curves (ARC). The time constant has been increased in order to improve SNR of the frequency shift signal. 96

Figure 57. The change in the contribution of the contact capacitance and the RLC circuit to the QTF signal. As the resonant frequency of the system shifts to the right, the capacitance increases its contribution to the measured signal.

Figure 58. Tip used for the studies of the HOPG sample, interrogating the interaction forces in the lower range domain (below $2 \mathrm{nN}$ ). The apex has a radius of approximately $50 \mathrm{~nm}$. FE-SEM image taken using $10 \mathrm{kV}$ accelerating voltage and $5 \mu \mathrm{A}$ emission current. 
Figure 59. SEM image of the probe mounted on a QTF. The distance from the edge of the tine is $109 \mu \mathrm{m}$ and the radius at the base is $15 \mu \mathrm{m}$. 102

Figure 60. Top: Approaching and retracting curves of one of the first approaches on the HOPG surface, showing hysteresis. Bottom: Approach only. The vertical line around the $100 \AA$ mark indicates contact with the adsorbed layer. The tunneling current keeps increasing after the jump while the elastic shear force remains constant. The $0 \AA$ mark indicates the end of the ARC. 103

Figure 61. The WGAS amplitude, normalized in percentage value, and the normalized SANM amplitude. The normalization factor is the amplitude of oscillation of the QTF, i.e. the WGAS amplitude signal (see Figure 62 ). The vertical line marks the onset of probelayer interaction. 104

Figure 62. Comparison between the un-normalized (left) and normalized (right) SANM signals. Each data point on the normalized trace is obtained by dividing the unnormalized value by the corresponding probe's amplitude (the WGAS signal). 105 Figure 63. Top: ARC showing hysteresis of the dissipation force and SANM phase. Bottom: The vertical line, signals contact with the layer. 106 Figure 64. Retraction curves of all signals. The vertical line shows the change in the slope of the elastic force. However the change equivalent to the initial jump does not appear. This is considered evidence of viscous character of the adsorbed layer. The tunneling current is in $\mathrm{nA}$. 107

Figure 65. Left: Comparison between the SANM amplitude and the frequency shift for the first approach process obtained after peeling off the HOPG layer. Right: Comparison between the frequency shift of the WGAS signal and the elastic force. 109

Figure 66. After several trials the ARCs start displaying no hysteresis in their interacting forces. The vertical line at around $150 \AA$ indicates interactions at the air-layer interface. Notice the existence of elastic force interaction $50 \AA$ before there is a tunneling signal. The vertical line around $90 \AA$ indicates a transition to solid-like interactions. 110 
Figure 67. The SANM amplitude increases before the onset of tunneling current by approximately $30 \AA$. A correlation between this SANM amplitude and elastic force is observed.

Figure 68. Dissipation force curve showing a marked increase in dissipation as the SANM phase becomes stable. The dissipation is now larger than the elastic force. A sign of medium change (or probe deformation) is seen around the $50 \AA$ mark of the dissipation curve.

Figure 69. The ARCs show a repeatable pattern after severa cycles. 113

Figure 70. The SANM amplitude and the dissipation curve showing different inflexion regions. While the SANM amplitude displays a sharp change in the slope, the dissipation force has an exponential behavior (as will be seen in section 6.1.F) around the $130 \AA$ mark.

Figure 71. SANM amplitude and elastic force showing a remarkable correlation. Similar to the SANM (and contrary to the dissipation in Figure 70) the elastic curve has a sharp change: its slope is constant before and after the transition at around $130 \AA$. 114

Figure 72. The WGAS amplitude shows a long interaction range of about $450 \AA$ after several ARCs. The tunneling current does not present a clear air-layer transition, but its linear exponential behavior starts at the $360 \AA$ mark. The vertical lines mark different transition points for the tunneling current.

Figure 73. The SANM phase changes by approximately 90 degrees. The change correlates well with the start of the exponential behavior of the dissipation force. The vertical lines follow the transitions observed in the tunneling current (Figure 72) showing weakly (but existent) correlations. 115

Figure 74. Comparison between the elastic force initial and subsequent curves. The force starts from longer distances as the number ARCs increases, in agreement with the higher interaction distances experienced with higher radii probes[75]. 116

Figure 75. The same case occurs with the dissipation force. These curves present a higher curvature radius, when compared to the elastic force. 117 
Figure 76. Top: Elastic and dissipation forces. Notice the difference in the inflexion. Bottom. Natural logarithm of the $\mathrm{k}$ and $\mathrm{b}$ for the subsequent curves. Both slops are constant for a short between $100 \AA$ and $50 \AA$. Below $50 \AA$, the behavior of both $\mathrm{k}$ and $\mathrm{b}$ is linear (and thus the natural logarithm arcs at that point). It is an indication of elastic contact between probe and sample as seen in nano-indentation processes. 118 Figure 77. Change in phase from the SANM acoustic sensor at the onset of mechanical coupling from the probe. The noisy signal before interaction is due to the weakly locked phase between the SANM sensor and the QTF. 119 Figure 78. Comparison between the SANM amplitude and the dissipation force for the first process with no hysteresis (transition curve). The SANM and dissipation force signals show different trends. 120

Figure 79. The correlation between the SANM amplitude and the elastic force of the transition curve. 121

Figure 80. Comparison between SANM amplitude frequency shift in the transition curve (hysteresis becomes negligible). The rest of the curves for this process are show in Figure 66 through Figure 68. 121

Figure 81. Probe used for the mica approach process. 124

Figure 82. The first ARC on mica in air. Open markers are the retraction curves. The oscillating pattern repeats every $5 \AA$ approximately. Right before the zero both signals increase suddenly indicating the end of the frequency modulation regime. 126 Figure 83. SANM amplitude and the dissipation force for the same curve in Figure 82. The periodic peaks are similar to the ones found in the elastic force and WGAS amplitude. The SANM presents no measurable signal at this point. The dissipation force signal shows the same periodic interaction peaks but the average dissipation force is still zero. The bottom sensor does not seem to record any oscillation either. 127 Figure 84. Curve showing reduced hysteresis. After several ARCs the curves the SANM Amplitude and dissipation force showing reduced hysteresis. 128 
Figure 85. SANM Amplitude and dissipation force showing reduced hysteresis as interaction between probe and sample happens repeatedly. 129

Figure 86. Averaged result of continuous ARCs. One feature is the absence of the jump to contact, evidence of an increase in layer thickness. 130

Figure 87. Elastic force compared with the SANM amplitude. No evidence of SANM signal, but the sudden jump to higher forces is noticeable at the end of the approach.

Figure 88. Comparison of length of interaction after several processes. This is believed to be caused by the change in the probe-surface contact area. 131

Figure 89 The logarithm WGAS elastic constant has a constant slope for approximately $15 \AA$ before dramatically increasing. The logarithm of the dissipation force showing a constant slope, evidence of viscous forces.

Figure 90. A volume of $1 \mathrm{ml}$ of de-ionized (DI) water placed on the silicon and cleaved mica used in the measurements. Both surfaces show hydrophilic behavior. 133 Figure 91. Probe used in the process on hydrogen terminated silicon. 135

Figure 92. Comparison between normalized and unnnormalized SANM amplitude signal. The elastic shear force shows the characteristics exponential behavior indicating the viscous drag force of the layer. 136

Figure 93. Left: The SANM phase shows a change which seems to indicate mechanical coupling with the with the surface. Right: The tunneling current starts at apporximately at the $350 \AA$ mark. 136

Figure 94. The natural logarithm of the dissipation and elastic constants in the first averaged set of approaches for silicon. The slope shows an approximately constant $b$ after the onset of the tunneling currentand (around the $350 \AA$ mark). Notice how both curves have an approximately constant slope, only to experience changes at around the $25 \AA$ mark. 137

Figure 95. Comparison between normalized and unnnormalized SANM amplitude signal for the second averaged set. The SANM normalized amplitude (left) seems to increase in 
the last $200 \AA$. SANM amplitude without normalization (right) shows no evidence increase. 138

Figure 96. The WGAS amplitude shows an interaction region of at least $1000 \AA$. The last $50 \AA ̊$ show a positive change in the slope, indicating a transition to a different medium. The tunneling current starts showing a linear behavior at around $250 \AA$ from the arbitrary zero. The SANM phase shows a small change which seems to indicate a soft contact with the surface. 139 Figure 97. The natural logarithm of the elastic constant and dissipation constant in the second set of averaged approaches for silicon. The erratic behavior before the onset of the tunneling current ( $250 \AA$ mark) could be attributed to the thick layer found on the silicon surface. 139 
LIST OF ACRONYMS

AFM Atomic Force Microscopy

AM-SPM Amplitude Modulation Scanning Probe Microscopy

ARC Approaching and Retracting Curve

ARCP Approaching and Retracting Curve Program

CQO Crystal Quartz Oscillator

DI De-ionized

DMA Direct Memory Allocation

FM-SPM Frequency Modulation Scanning Probe Microscopy

HRS High Resolution Stage

NSOM Near Field Scanning Probe Microscopy

PID Proportional Integral Differential

SANM Shear Acoustic Near-Field Microscopy

SFA Surface Force Apparatus

QTF Quartz Tuning Fork

WGAS Whispering Gallery Acoustic Sensing 


\section{CHAPTER 1}

\section{The SCIENCE and TECHNOLOGY ISSUES UNDERLYING THIS THESIS}

\subsection{The unique physical properties of mesoscopic fluid films}

The physical properties of mesoscopic fluids found adsorbed at substrates or confined between solid surfaces differ greatly from those measured in their bulk form[1]-[3]. For example, the effective shear viscosity is enhanced, viscoelastic relaxation times are prolonged[4], they reveal different phase diagram[5] (boiling at lower temperatures, ice formation at room temperature[6], [7]), the so called hydrophobic interaction manifests at distances well beyond commonly accepted molecular interaction ranges[8], interfacial adhesion[9], molecular transport in nanoestructures[10], [11], molecular assembly of particles in liquid water[12], [13] and filtration[14]. But the physics of such a unique dynamic behavior displayed by mesoscopic fluid films still lacks a comprehensive description.

For instance, there is no adequate theoretical explanation yet for the claimed ice formation at room temperature in meniscus bridges[5], [15]. Still, experimental results obtained by scanning a graphite sample with a sharp probe $(2.5 \mathrm{~nm} / \mathrm{s}$ scan velocity and 5-30\% relative humidity $(\mathrm{RH})$ range), reveal pronounced stick-slip motion in the force loops with a period of $0.4 \pm 0.1 \mathrm{~nm}$, very different from the $0.25 \mathrm{~nm}$ distance typical for graphite but close to the $0.45 \mathrm{~nm}$ lattice constant of hexagonal ice[4], [6]. Also, the measured two-second time response of the meniscus (to a sudden change in the motion of the probe) is too long compared to the expected microseconds to 
milliseconds for the formation of a capillary neck via adsorption of water molecules from the water vapor at room temperature and RH $50 \%[16]$, [17]; but if we accepted that the condensed water meniscus "immediately" converts into ice, the time scale for ice rearrangements by plastic deformation would be much slower than liquid water[4]. Ice formation at room temperature between a tip and a flat gold surface under the effect of very low electric fields has also been reported[7]; and that once formed confinement may not be needed[15].

On the other hand, the nature of the fluid itself could play a role in their behavior. Films made of water molecules and films made of larger organic molecules (like octamethylcyclo-tetrasiloxane, OMCTS) could display different behavior. Maybe their peculiar group organization (further influenced by the neighboring substrate geometry) would play an important role. Water, for example, with its ubiquitous hydrogen bonding, is considered a giant molecule, conforming around substrates. Such a conformational ordering depends on the space available between two interacting substrates and quantified in term of its entropy. The latter would then have a crucial effect on the water-mediated interaction between solid surfaces; for if a lower density in the gap were more favorable in entropic terms, then an attractive force between the solid surfaces would result. In fact, the latter argument is typically used to explain the working mechanism behind the long-range (sometimes greater than $20 \mathrm{~nm}$ ) hydrophobic interaction[18]. Molecules like OMCTS (non-polar, quasi spherical, $9 \AA$ 
diameter), on the other hand, do not have such a cooperative gigantic organization; hence its dynamic response to confinement between solid surfaces may be different.

\subsection{The relevance of gaining a better understanding of mesoscopic films properties}

Studies of mesoscopic fluid films are relevant to technological areas like adhesion, wetting processes, and nanotribology in general[19] (as there has been considerable effort to develop thin-film coatings to eliminate capillary adhesion between components, and to reduce corrosion and wear)[20], [21]. They are also important for understanding the rigidity and flow behavior of granular materials[22], [23] and ceramics[24], in which deformation occurs by means of the shearing of thin interfacial layers at grain or phase boundaries. They can also lead to the implementation of a better modeling of bio-membranes[25], gain understanding of water mediated hydrophobic interaction (which mediate the synchronized traffic of fluids in biological membranes)[25], and potentially shed lights on the dynamics of proteins' folding unfolding processes.

This thesis aims at the development of a new acoustic metrology tool that could help to attain a better understanding of interfacial tribology phenomena, focusing on the role played by the fluid film trapped between solid surfaces in relative motion. In particular, we are interested in understanding how does wear-free friction work. Could it be that energy is dissipated via phonons (i.e. energy transmitted in the form of sound waves originated in the confined liquid and coupled to the sliding substrate?)[25], [26]. 
Support for this view can be found in molecular dynamics (MD) simulations studies of the phononic mechanism[27], [28]. Briefly, a rigid crystalline substrate produces a periodic potential $V_{\text {substrate }}(z, \boldsymbol{r})$ on the adsorbed molecules (here $z$ is the coordinate along the axis perpendicular to the substrate surface; $r$ is the lateral coordinate.) As the substrate shakes laterally and harmonically, it produces deformations on the adsorbate. If the layer followed a similar harmonic motion (i. e. no deformation, as is likely to happen if the adsorbed layer were in a solid state), then there would be no dissipation of energy. But if the adsorbed layer were close to a liquid state, anharmonic oscillations (i.e. out of phase with the substrate motion) would lead to a draining of energy[29]. That is, in the phononic mechanism, dissipation of energy arises from substrate-induced deformations in the adsorbate and anharmonic coupling between phonon modes[30].

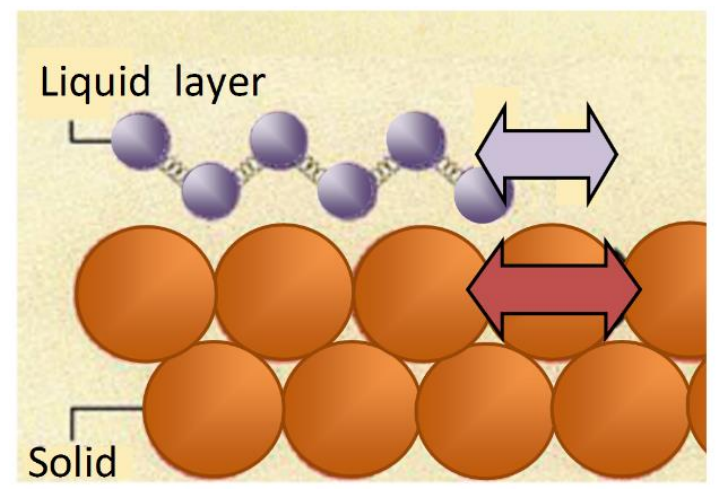

Figure 1. Energy dissipation in interfacial friction phenomena by the phononic mechanism. As the solid substrate oscillates harmonically, it produces deformation on the liquid layer; anharmonic motion responses in the liquid (i.e. out of synchronization with the substrate motion) lead to a net dissipation of energy[36].

This thesis pursues measurements of the phase lag between the sliding solid surfaces and the response of the fluid trapped in between. The implementation of 
Phase-lock-loop acoustic feedback technique to measure this phase lag is described herein. The studies of fluid films in this thesis includes the use of substrates with different hydrophobic and electrical conductivity characteristics, as a way to evaluate the influence of the nature of the substrate boundaries on the fluid dynamics response to shear motion. These initial measurements could serve as a seed for future studies aiming at interrogating the nature of the hydrophobic force itself. The latter refers to the interaction between molecules or between surfaces in water, often stronger than their attraction when in free space[31]. (The brief description that follows is to provide some context to the results of this thesis to be presented in the next chapters). Although the 'hydrophobic' term means 'water fearing', the interaction between a hydrophobic molecule (typically a non-polar molecule incapable of forming hydrogen bonds) and water is actually attractive (due to the van der Walls forces, as occur with any other two molecules with induced electrical dipoles); but hydrophobic interaction, it turns out, is simply stronger than what is expected from traditional continuum models (van der Waals, DLVO[32]). Its nature, however, is a matter of controversy; after 30 years of efforts, the search for the hydrophobic force law continues)[8]. Still, a trend of consensus exists that the force between hydrophobic surfaces manifests differently within three different regimes[33], [34]:

i. From 100 to 1000 of angstroms, the attractive force is dominated by electrostatic domains present on the approaching surfaces and/or the bridging of vapor cavities[35] (described within the context of capillary forces)[36]. 
ii. In the 15 to 150 angstroms range, a long-range hydrophobic force is possibly due to the 'proton-hopping' polarizability of water. (Proton-hoping in water is inferred from the fairly rising-trend of the water's dielectric constant upon freezing, contrasting the abrupt fall of $\varepsilon$ observed in other polar liquids. An abrupt fall indicates that the contribution to $\varepsilon$ from thermal rotation of the dipolar molecule has stopped. The high polarizability of water is believed, then, to arise from the proton hopping along the H-bond network[33]).

iii. In the range below 10-15 angstroms a short-range hydrophobic force is related to water structuring effects associated with surface-induced changes in the orientation and/or density of water molecules and $\mathrm{H}$-bonds at the water-hydrophobic interface. Some consider this regime to be the true range of the hydrophobic interaction. Since the force is typically detected by monitoring the deflection of the spring supporting one of the surfaces, this short-range regime of strong interaction is typically inaccessible to high lateral resolution tools like atomic force microscopy (AFM). The shortcoming comes from the fact that an AFM-probe is inherently unstable, as the cantilever will snap into or out of contact with a surface whenever the gradient of the force between the tip and the sample exceeds the cantilever spring constant $k$ (of few tens of $\mathrm{N} / \mathrm{m}$ )[33]. In contrast, the newer shear-force acoustic near-field microscopy and whispering gallery acoustic sensing techniques employed in this thesis does not have such a limitation (it uses a probe with effective spring constant practically infinite along the vertical direction, and $\sim 20,000 \mathrm{~N} / \mathrm{m}$ along the lateral 
direction, as described herein). Hence, SANM is more suitable and very promising for interrogating the hydrophobic interaction at very short probe-sample separation range.

\subsection{Context: The emphasis on the study of interfaces}

In spite of a standing interest, the dynamics, thermodynamics, and the molecular structure of fluids confined to nanometer-sized regions are not yet well understood at the fundamental level. Efforts to elucidate this problem can be traced to developments in the colloid science field (the study of heterogeneous systems that behave as springy liquid and sticky solid, hence no classifying within the standard solid, liquid, or gas phases).

For the past 70 years, the theoretical framework guiding the progress in this area of soft condensed matter has been dominated by the Deryaguin, Landau, Verwey, Overbeek theory (a bottom-up approach theory based on the addition of pair-wise interactions subsequently integrated all over the constituents parts)[37], and later by the Lifshitz theory (dealing with interactions across vacuum[37], subsequently extended to include interactions between the surfaces of colloidal particles separated by an intervening liquid medium[38]) a top-down approach where the measured bulk electrical susceptibilities are incorporated into the molecular constituents interactions. Both approaches attained notable achievements but applied to relatively limited cases. For the majority of cases under realistic experimental conditions (no uniform density, 
granular media, high concentration of ions), the models become mathematically more difficult, or specific problems had to be fitted with so many parameters that the power of making prediction for different situations is lost. As a matter of fact, the field of colloidal science has been experiencing drastic new revisions in the last decade, as revealed in the refreshing reference by B. W. Ninham and P. Lo Nostro[39]. The revisions respond to the need of better addressing, in particular, the heterogeneous characteristic of real substances, the role of ions (Hofmeister effect)[16], [17], and the effects of cavitation[18], [20].

One aspect of these limitations is that the old thermodynamic theories have been constructed based on theoretical abstractions applicable to homogeneous materials (to make the theory more mathematically tractable). To remediate this situation, part of the new revision in colloidal science focuses on the study of phenomena occurring near interfaces, where the gradient of density (or other thermodynamic properties) changes drastically only along the direction perpendicular to the interface. Such an ideal situation should display mechanisms similar to the way by which, for example, a liquid terminates at a boundary where it breaks into vapor; it is for this reason that the study of dynamic processes at interfaces is appealing. This point is pertinent to the objectives of this thesis, which aim at the study of mesoscopic films at surfaces (as detailed herein). 


\subsection{Standard surface characterization techniques}

For the characterization of phenomena occurring at interfaces, the surface force apparatus (SFA)[40] stands out for the pioneering results measuring forces between parallel surfaces separated by distances down to the angstrom level[41], [42]. In 1982, SFA measured an attractive force $F=0.14 \mathrm{e}^{-\mathrm{D} /(1.0 \mathrm{~nm})} \mathrm{N} / \mathrm{m}$ between two hydrophobic cylindrical surfaces of radius $R$, for separation distances $D$ in the $0-10 \mathrm{~nm}$ range[43]. Modified SFA versions allow studying the dynamic of fluids under oscillatory shear[44], [45]. Succinctly, the SFA uses lubricated contacts between atomically-smooth mica surfaces mounted on crossed half-cylinders that laterally slide against each other. The deflections of springs attached to the half-cylinders measure the friction forces[46] under static[47] and dynamic[48] conditions. Notice, however, the indirect nature of the measurement; the dynamic of the fluid is inferred from the mechanical response of the external spring. Also, inherent to its setup, the SFA does not provide lateral resolution close to the nanometer level.

Another important technique is quartz microbalance (QM), which exploits the low dissipation internal energy of a single crystal quartz to sense the slippery motion of adsorbed molecules, the latter causing a broadening in the frequency-response of the (5-10 MHz) crystal's resonance peak[49]. QM has revealed that, contrary to what a macro scale experiment interpretations would suggest, solid films slide more readily than liquid films[50]. The results have been interpreted in terms of the phononic friction mechanism[31], [51] (Figure 1 above). The technique, however, does not offer 
nanometer-sized lateral resolution, and it is limited to investigate relatively small friction forces so that sufficient sliding (of the adsorbed molecules relative to the QM surface) can occur.

Atomic force microscopy (AFM) brings atomic resolution to the analysis of surfaces[52]. A pyramidal-shape probe is dragged along the surface while its lateral bending, caused by the probe-specimen frictional force is monitored with a laser beam reflecting from the probe. The smaller the bending experienced by the probe, the lower the frictional force[53], [54]. However, similar to a surface force apparatus setting, the technique senses the effects that surface interactions cause on the probe only, while the response from the mesoscopic fluid (trapped between the probe and the substrate) is lost or inferred only from models. (The new SANM acoustic technique described below aims this shortcoming). The AFM probe also suffers from force calibration issues[55] and, particularly, from instabilities[56]. In fact, the spring constant of the AFM probe (being no so rigid, typically few tens of $\mathrm{N} / \mathrm{m}$ ) suffers from snap-in motion (abrupt downward deflection towards the surface) when the probe is being approached to the substrate, which makes difficult to interpret the probe's response, particularly at very short probe-sample distances where the interaction is stronger than what the AFM probe can discern. In contrast, the tuning fork probe used in the SANM is more robust and stable; the acoustic signal itself has greater sensitivity in the short range region closer to the surface, as it will be shown herein. 
Recent work using a quartz tuning fork SPM (QTF-SPM) system with frequency modulation[57] and amplitude modulation[58] to investigate the properties of the adsorbed layer on hydrophilic surfaces has shown the capabilities of the QTF-SPM technique. However, in none of the four techniques mentioned above the response from the fluid is probed directly.

Monitoring simultaneously the effects that surface interactions have on both the probe and the fluid layer would be advantageous, since a potential phase difference between these two oscillatory motions could reveal important working principles of interfacial (i.e. wear-less) friction. Aiming to fulfill such a capability motivated the development of SANM.

\subsection{Shear-force acoustic near-field microscopy (SANM)}

More recently, our group introduced Shear-force Acoustic Near-field Microscopy (SANM) for directly monitoring, via acoustic transducers, the dynamics of mesoscopic fluids trapped between two nanometer-sized solid boundaries under relative shear motion[59], [60]. SANM was demonstrated in 2005 independent of a similar experimental setting introduced in 1989 that aimed principally at lateral scanning imaging applications, with a working mechanism attributed to the dynamic changes of the gas surrounding the probe[61]. 

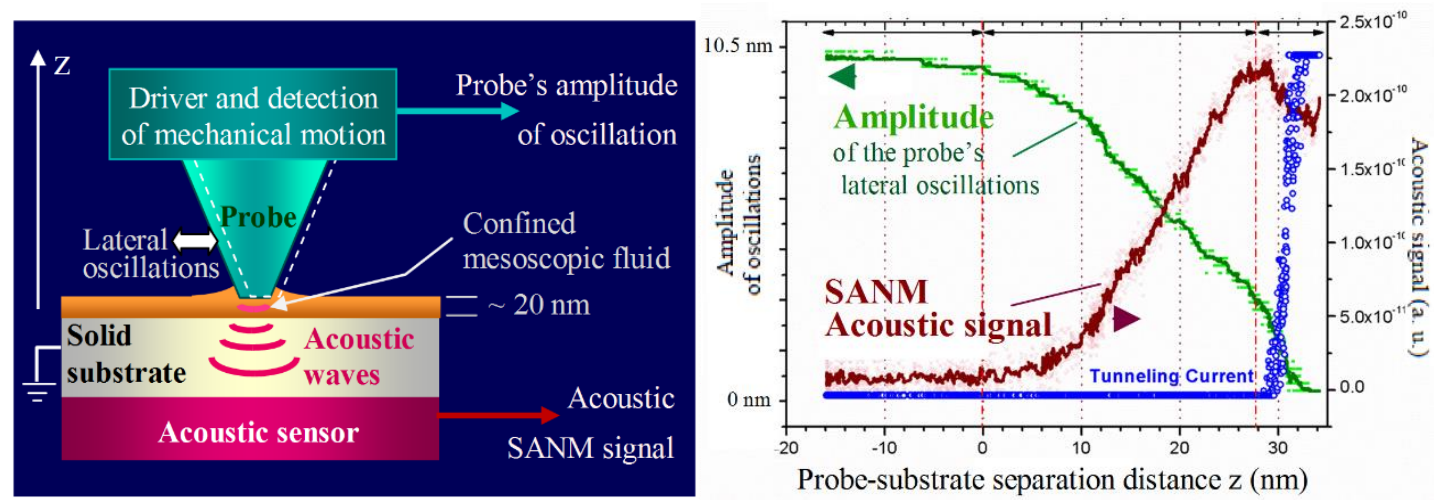

Figure 2. Monitoring the acoustic response from confined mesoscopic fluid trapped between solid surfaces in relative motion. Left: The apex of a laterally-oscillating probe is brought into shear interaction with an adsorbed mesoscopic fluid film, engendering an acoustic wave that couples to the substrate, travels through the substrate bulk, and is finally detected by an acoustic transducer. Right: A metallic probe and metallic sample monitors the onset of current (blue trace). Notice the detection of acoustic SANM-signal even when there is not tunneling current.

In contrast, SANM exploits the fact that an acoustic transducer (attached underneath a stationary substrate, as shown in Figure 2) has sufficient sensitivity to detect acoustic waves engendered at the matrix of the fluid layer trapped between the oscillating nanometer-sized apex-probe and the sample surface; such capability had been overlooked in the past. The acoustic waves are driven by the apex-probe, whose mechanical oscillations are also monitored (simultaneously) in the SANM setup. This dual capability offers an opportunity to measure the eventual phase-lag of the fluid's motion relative to the moving solid apex boundary, which could contribute in understanding the adhesive irreversibility (or hysteresis) involved in tribology phenomena, as it was addressed in Section 1.2 above. The phase-lag measurement 
requires the implementation of frequency modulation and its decoupling properties, underlining its impact in the implementation of the SANM-WGAS integrated SPM system. The characteristics of frequency modulation are presented in the next chapter in the context of other scanning probe microscopy developments. 


\section{CHAPTER 2}

\section{FREQUENCY MODULATION NEAR FIELD ACOUSTIC MICROSCOPY in the CONTEXT of SCANNING PROBE MICROSCOPY DEVELOPMENTS}

The near-field acoustic techniques used in this thesis to characterize the dynamics of mesoscopic fluid films constitute an outgrowth of a large family of scanning probe microscopies that are nowadays widely used to characterize materials with high lateral resolution. This was hinted in the last section of chapter 1 above. In this chapter we provide the technical context in which these new acoustic techniques were developed their relevance as a sensing mechanism to characterize materials at the nanoscale and the differences with existent acoustic SPM technologies. In passing, it is interesting to highlight the disparity between the size of the analyzed region $\left(10 \times 10^{-9} \mathrm{~m}\right)$ and the wavelength of the operating acoustic wave $\left(10^{-1} \mathrm{~m}\right)$.

This chapter includes a brief description of the particular SPM techniques that have been influential to the development of WGAS and SANM, highlights of the importance of the quartz tuning fork as a low energy dissipation sensing element, and a rationale of the benefits of adding frequency modulation capabilities. This broad view will pave the way for the following chapter in which the technical implementation of the new acoustic techniques are described in more detail. 


\subsection{Scanning probe microscopy}

The invention of the scanning tunneling microscope (STM) by G. Binnig and $\mathrm{H}$. Rohrer in 1982[62] opened the door for the development of a family of techniques known today as scanning probe microscopy (SPM). The STM's principle of operation rests on the quantum tunneling effect that occurs when a voltage-biased sharp conductive probe is brought close to an electrically grounded conductive surface. The monotonic dependence of the tunneling current on the probe-sample distance allows implementing a tip-sample distance control. Keeping the tunneling current constant while scanning the probe laterally across the sample allows topographic images with atomic resolution. With the advent of the atomic force microscope (AFM)[63] the range of samples available for characterization expanded to non-conductive surfaces, thus revolutionizing the investigation of local surface properties. The addition of dynamic control and the introduction of an instantaneous frequency tracker by Durig[64] allowed for the implementation of harmonic analysis and the creation of imaging modes based on the probe tapping on the surface, thus increasing the analytical capabilities of SPM systems.

The common denominator found in every type of SPM is a sharp probe placed in close proximity to the surface region under test, and an electronic system continuously tracking a specific physical parameter (tunneling current, probe amplitude of vibration, capacitance, etc.) to control the probe-sample distance. 
Their ability to image at atmospheric pressure, room temperature and the non-invasive (and therefore non-destructive) character of non-contact modes has made SPMs technically appealing for the development of acoustic-based imaging and characterization of soft samples.

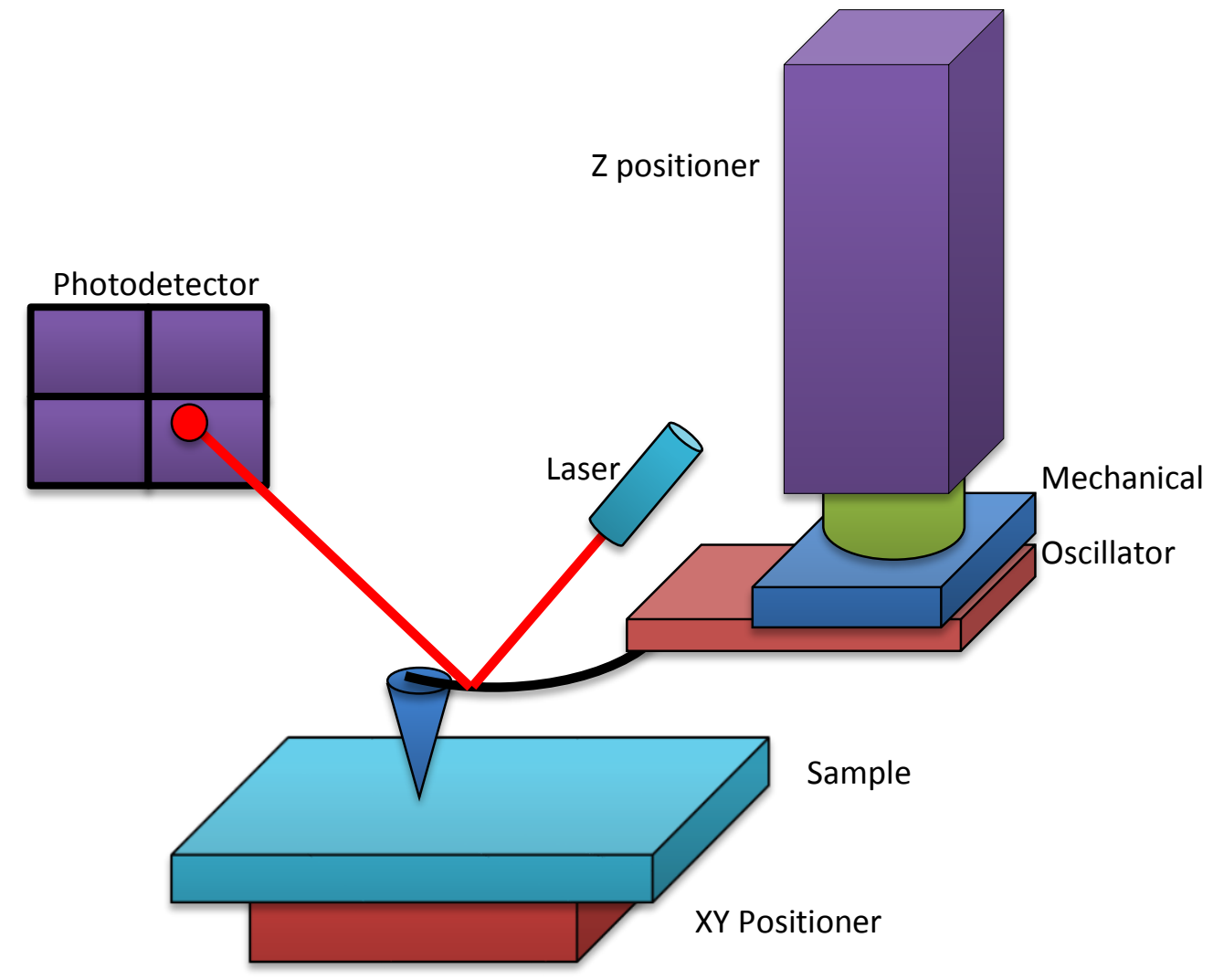

Figure 3. Atomic Force Microscope experimental setting diagram.

\subsection{Quartz tuning fork scanning probe microscopy (QTF-SPM)}

A critical aspect in SPM is the instrumentation sensitivity to detect the cantilever's response as a function of the probe-sample distance. In the case of AFM, 
when the probe interacts with the sample in contact mode, it bends and this deformation is what it is measured. When in non-contact mode, the probe is oscillating and the interaction range is longer. The sensitivity decreases along with the amplitude. The stiffness $k$ (in $\mathrm{N} / \mathrm{m}$ ) of a typical AFM cantilever is inversely proportional to its force sensitivity and while low values are used to reach sub-nN detection floors it means that unwanted and significant modifications of its motion dynamics cannot be prevented, which compromises its metrology capabilities.

The use of a quartz tuning fork (QTF) as a transducer for SPM was introduced in 1995[65]. A QTF combines a high stiffness ( $k$ above $2000 \mathrm{~N} / \mathrm{m}$ ) with very low energy dissipation (mechanical factor $Q$ greater than 1500), which allows using sub-nanometer oscillation amplitude and relatively low effective forces. The QTF can be used in shear force mode, by oscillating the probe parallel to the sample, which makes it suitable to probe to the apparently high viscoelastic nature of the regions close to the surface. Due to the stiffness of the QTF the 'jump to contact' phenomenon (typically observed in AFM experiments) does not affect the position of the probe in the acquisition of approaching and retracting curves (ARC) used in this thesis to characterize the mesoscopic fluid layer.

Introduced by Gäunther, Fischer and Dransfeld[61] and later developed by Karrai and Grober[65], the crystal quartz oscillator (CQO) with an XY cut, referred to here as quartz tuning fork (QTF), has been used for tip-sample positioning control in Near-field scanning optical microscopy (NSOM) since 1995[66]-[69]. The single harmonic oscillator 
(SHO) model is often used to account for the motion of the QTF. The model, it turns out, provides an excellent qualitative and good quantitative approximation for the motion of the QTF in the absence of first principles calculations, probably due to its intrinsic internal low energy dissipation. One typically associates an equivalent RLC electrical circuit to the mechanical simple harmonic oscillator model, which incorporates an intrinsic capacitance $C_{0}$ of the QTF. This is well known model for CQOs, consisting in a series connection of equivalent RLC elements (resistor, inductor, capacitor) in parallel with a capacitor $C_{0}$, which relates to the mechanical properties of the QTF as laid out by Butterworth-Van Dyke model[70], [71]. Owing to its high stability, precision and low power consumption this type of QTF has become a valuable basic component for SPM amplitude modulation and frequency modulation measurements as well.

As a piezoelectric, there is a linear relationship between the amount of charge and the corresponding deflection of the QTF tines. The charge separation $Q$ on the electrodes of the piezo material per mechanical deflection can be obtained experimentally by measuring the electromechanical coupling constant $\alpha$ [72], which is the amount of charge per change in deflection of the tines in meters $([\alpha]=\mathrm{C} / \mathrm{m})$. The experimental details for the calculation of $\alpha$ is presented in chapter 3. Here we present a derivation of the equation that relates the equivalent capacitance $C$ of the QTF, found through fitting, and its equivalent spring constant $k$ to the coupling constant $\alpha$. Using a relationship from the mechanical model[73], $C=\frac{2 \alpha^{2}}{k}$ and rearranging the terms results in, 


$$
\alpha=\sqrt{\frac{k C}{2}} .
$$

Finally the harmonic approximation[73] gives,

$$
\Delta I_{R M S}=4 \pi f \alpha \Delta x_{R M S}
$$

which will be used to calibrate the motion of the QTF.
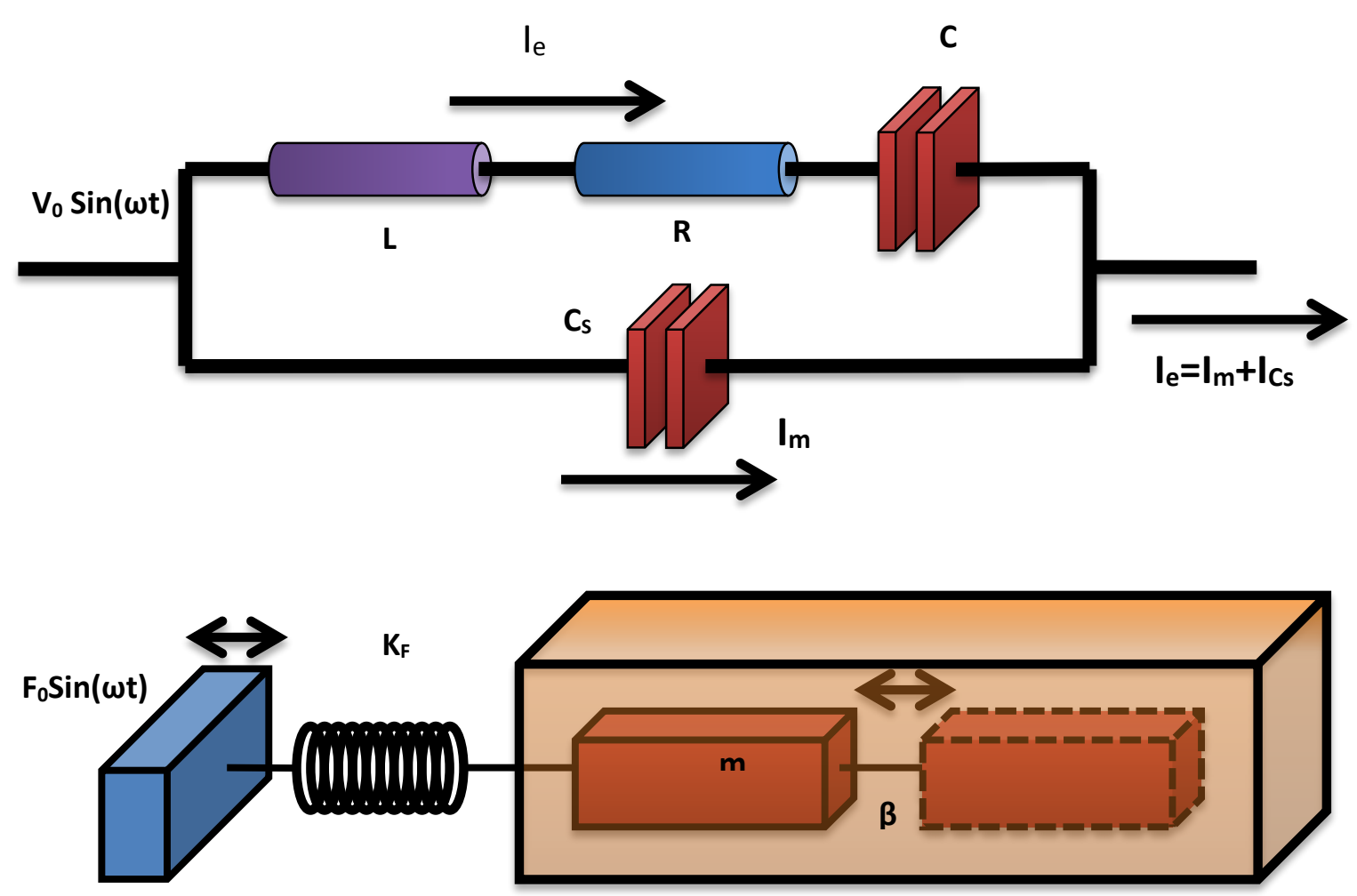

Figure 4. RCL circuit equivalent to the mechanical Butterworth-K. S. Van Dyke QTF model. 
A direct approach for the calculation of $k$ was shown by Karrai [72] where for a rectangular cantilever of length $L$, width $w$ and thickness $t$, the effective spring constant is given as,

$$
k=\frac{E}{4}\left(\frac{t}{L}\right)^{3} w
$$

where $E$ is the Young's modulus of quartz. This equation has been shown to underestimate the value of $k$ [74]. There are three well known technique to corroborate the value of the stiffness $k$ [74]: optical interferometry, mass loading and thermal noise measurements. The latter one provides an in-situ measurement method and it will be explained in more detail in chapter 3.

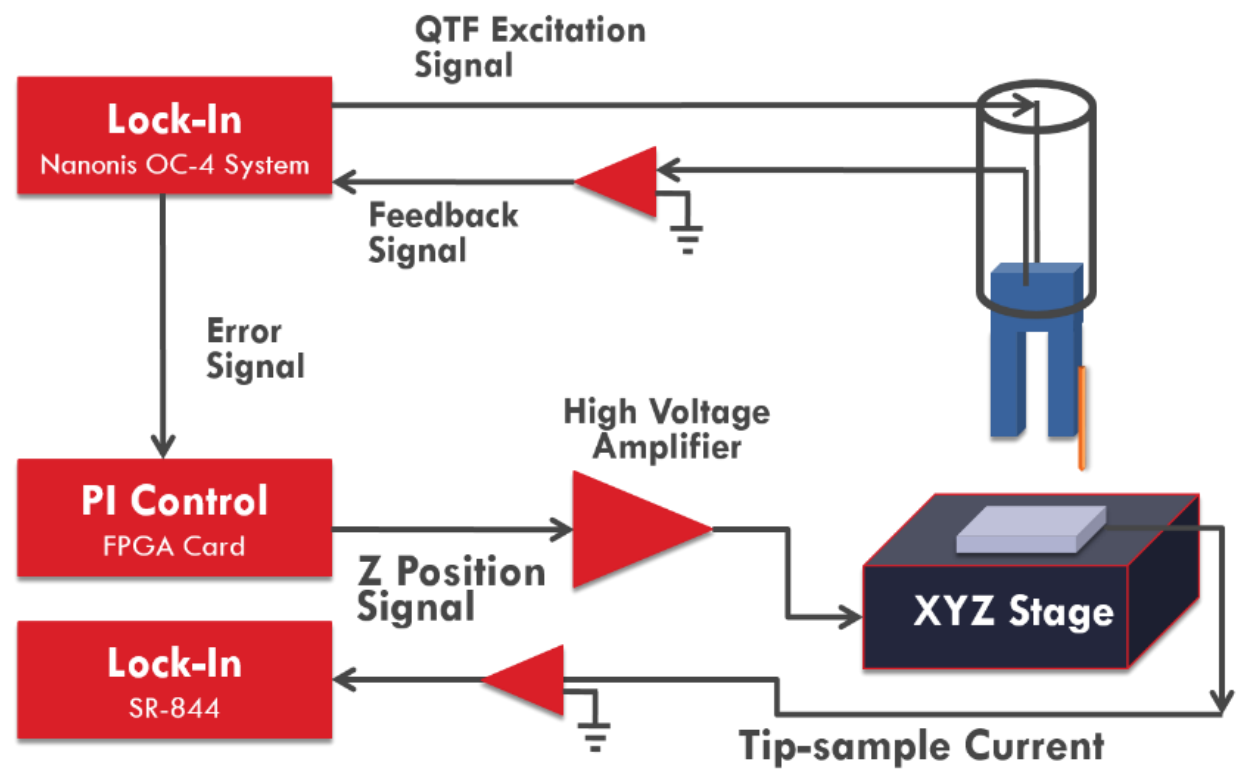

Figure 5. Quartz Tuning Fork Scanning Probe Microscopy (QTF-SPM). 
Figure 5 shows the basic arrangement for a basic SPM system. The diagram shows the particular case in which a QTF is the sensor used to control the vertical position of the probe, but the diagram will be equally valid if the QTF is replaced by a cantilever and its corresponding optical detection setup (such as in atomic force microscopy), or by simply a sharp metallic probe with a corresponding tunneling current detection setup (such as in scanning tunneling microscopy).

\subsection{Whispering gallery acoustic sensing (WGAS) feedback}

In QTF-based SPM the control of the probe's vertical position is implemented by electrically driving the QTF with an ac voltage ( 1mV rms) and synchronously detecting its corresponding admittance[75]. However, the reactance of the TF's inherently capacitance ends up convoluting the electrical detection of the QTF's tines amplitude. This capacitance prevents the operator from determining the exact state of the TF's mechanical motion, including, for example, whether or not the probe has been brought to a complete rest by the interactions between the tip and the substrate; even if in that latter state the QTF's admittance signal is different from zero. Precise determination of whether or not the tip is at rest provides pivotal information about the ability of shear forces to influence the probe's motion, an important aspect for friction studies at the nanometer scale. Even though alternative methods have been introduced to compensate these shortcomings[76] simpler methods to directly attest the state of motion of the probe are desirable. 
Whispering-Gallery Acoustic Sensing (WGAS), introduced by our group in 2011[60], offers an alternative for establishing a direct correlation between the acoustic signal and the TF's mechanical motion, hence providing an opportunity to make a more direct assessment of tip-sample interactions when integrated into a QTF-SPM.

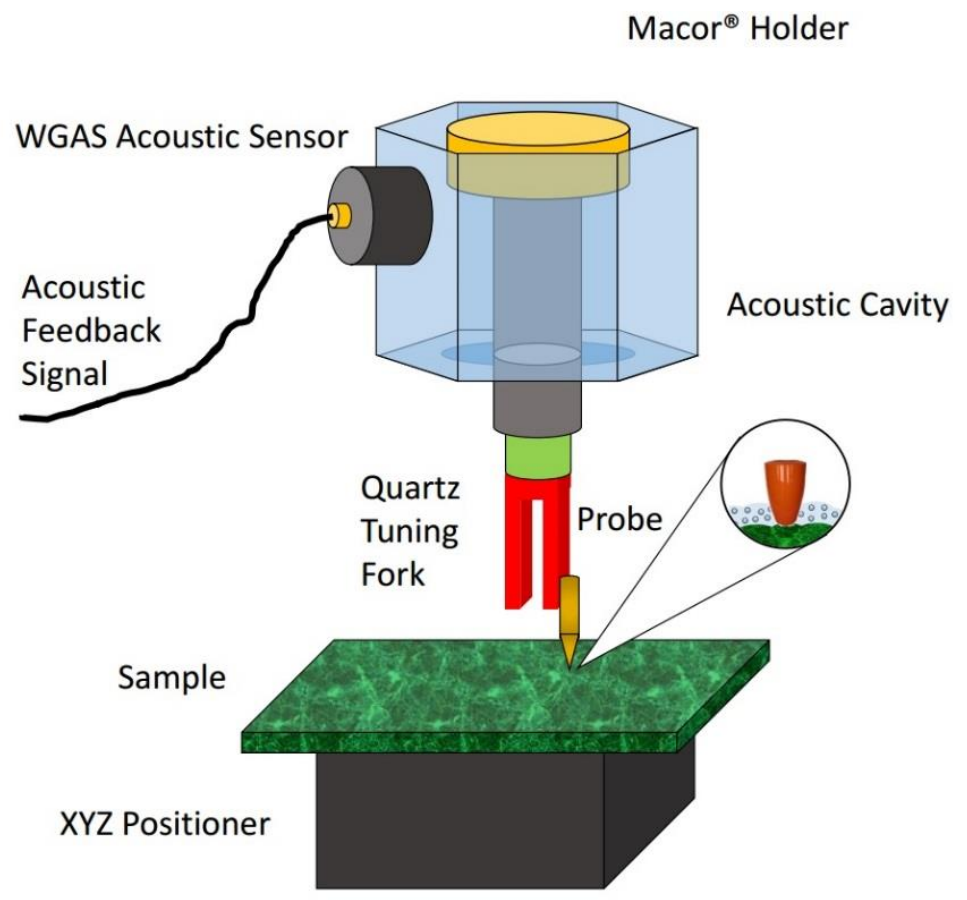

Figure 6. Whispering Gallery Acoustic Sensing (WGAS) implementation for monitoring the mechanical lateral oscillations of the electrically driven $32 \mathrm{kHz}$ quartz tuning fork to which a probe is firmly attached. The amplitude of the oscillatory motion of the tines of the QTF sets an acoustic wave that travels up to the cavity where a series of standing acoustic waves are established. An acoustic transducer is judiciously located at the periphery of the cavity to maximize the detected signal.

The acoustic detection approach circumvents the shortcomings associated to the QTF's capacitance that arises in purely electrical detection settings The simplicity of AWG 
resides in using the existent microscope's frame also as an acoustic cavity where standing waves (generated by the mechanical motion of the electrically driven QTF) are detected in a whispering-gallery type modality; i. e. similar to the faint, but still clear, conversations that can be heard at the galleries of a cathedral from a source located at extraordinary distances[77]. Exploiting constructive interference affect established in the acoustic cavity, WGAS can sense the QTF's tines lateral motion down to nanometersized amplitudes.

\subsection{Shear-force acoustic near-field microscopy (SANM)}

SANM is an acoustic-based technique developed to characterize the dynamic response of films adsorbed on solid substrates[59], [78], [79]. SANM capitalizes on the water layer (often containing molecules other than water) that gets adsorbed on a substrate at ambient conditions. In the case of water, it forms in part due to the hydrophilic nature of the sample's surface; but many other physical process may be at play in its formation or affect its physical structure. The layer's thickness can reach several nanometers depending on the humidity level, the material type and relative hydrophobicity of the substrate.

When a laterally oscillating probe is brought closer to the sample and gets in contact with the adsorbed fluid-like layer, the probe-fluid interaction sets an acoustic wave whose intensity, has been found, depends on the probe-sample distance. An acoustic sensor, attached to the bottom of the sample, is used to detect the acoustic

signal. The probe-sample dependence of the acoustic signal suggest a corresponding 
distance dependence of the fluid layer viscoelastic properties. Thus, SANM provides a new avenue to investigate the role of mesoscopic fluid-films on the tip-sample interactions.

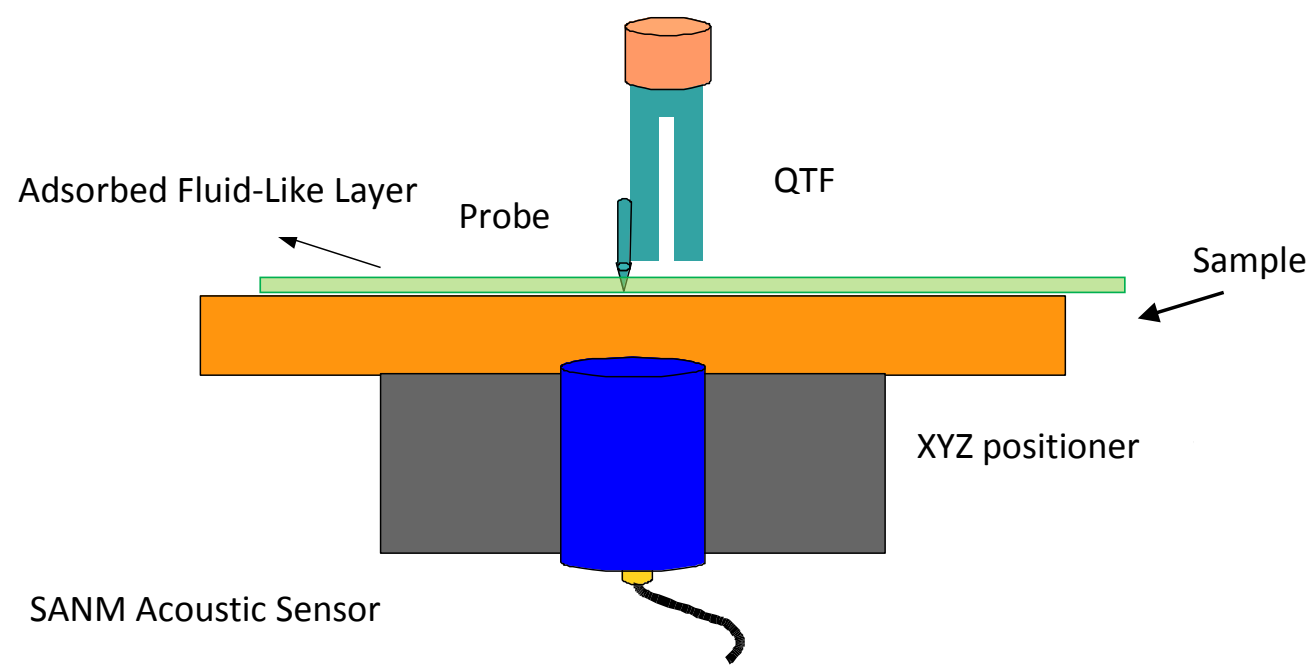

Figure 7. SANM experimental setup. The lateral oscillations of the probe, upon its interaction with the adsorbed fluid layer, engenders an acoustic wave, which is detected by the acoustic transducer located at the bottom in mechanical contact with the sample.

The new method differs from other acoustic techniques, where the probe dithers perpendicular to the sample or is kept stationary while an ultrasonic wave is applied to the sample. They are conceived to literally 'knock' the probe against the sample, which may have responded to the accepted notion of the poor acoustic coupling at a solid-liquid interface. Such operation contrasts the mechanism of the SANM where the acoustic signal is generated without having the probe to mechanically 
touch the solid substrate. In a SANM system, however, the excitation is right there at the fluid very close to the solid-liquid interface, a region of, it appears, very strong viscoelastic properties, which contributes to engender acoustic signals more efficiently.

The SANM system is integrated with the acoustic WGAS technique (described above) that senses the QTF motion (to which the probe is attached). Hence, while the SANM acoustic sensor monitors the surface interaction effects on the fluid layer, the WGAS signal monitor the motion of the probe. Both signals (WGAS and SANM) are measured concurrently and appear sufficiently different so that they can be contrasted to give more insights than either one by itself. Thus, the tandem SANM and WGAS enriches the potential information to interpret the intervening probe specimen interactions. (In fact this is exploited in a system that also integrates frequency modulation, as addressed in the next paragraph). Retrospectively, SANM was conceived in the context of expanding the capabilities of Near-field Scanning Optical Microscopy[80] hence, from the very beginning it was not required to choose probe's oscillations of exceedingly large amplitude. Integrating both near field techniques into one system increases their characterization capabilities for opto-acoustic materials characterization.

\subsection{Frequency modulation in scanning probe microscopy}

The two acoustic techniques mentioned above, WGAS and SANM, were initially implemented by driving the QTF at a single fixed frequency. In this thesis, they both have been extended to frequency modulation operation. When the mesoscopic layer 
adsorbed to a surface at ambient condition interacts with the oscillating probe, dissipative and elastic responses occur. The dissipative force component will not produce a significant change in the resonant frequency of the QTF-probe system, but an elastic response from the fluid layer (or an eventual knock interaction with the surface) will. Tracking of the resonance frequency variation becomes then imperative for a better characterization, in real time, of the mesoscopic layer, in particular its viscoelastic properties. Indeed, since in the frequency modulation technique the probe is driven at resonance frequency at all times, a change in frequency (due to a variation in the probesample distance) can be ascribed to the elastic component of the surface interactions, while a change in amplitude to dissipative interaction components. The decoupling the conservative and non-conservative probe-sample interactions is accomplished by changing the excitation signal frequency so that the phase lag between the excitation voltage and the response of the QTF remains at 90 degrees (this is the essence of the phase-locked loop technique) at all times. If the QTF is always driven at its real time RF a decrease in the oscillations amplitude can be ascribed to the non-conservative components of the surface interaction (viscosity of the adsorbed layer, friction from the surface, etc.) as schematically suggested in Figure 8 . There is disagreement as to where the conservative and dissipative forces begin in the course of approach and retraction curves and on the nature of the intervening interactions forces. The general consensus is that the formation of the meniscus and other capillary forces are at action at $10 \mathrm{~nm}$ probe-sample distances regime. A more challenging task is to discern the nature of the 
forces in the 0 to $5 \mathrm{~nm}$ range where morphology of the probe, the material type, the presence of ions, or the ubiquitous hydrophobic interaction (if it exists) may be playing a role all at once.

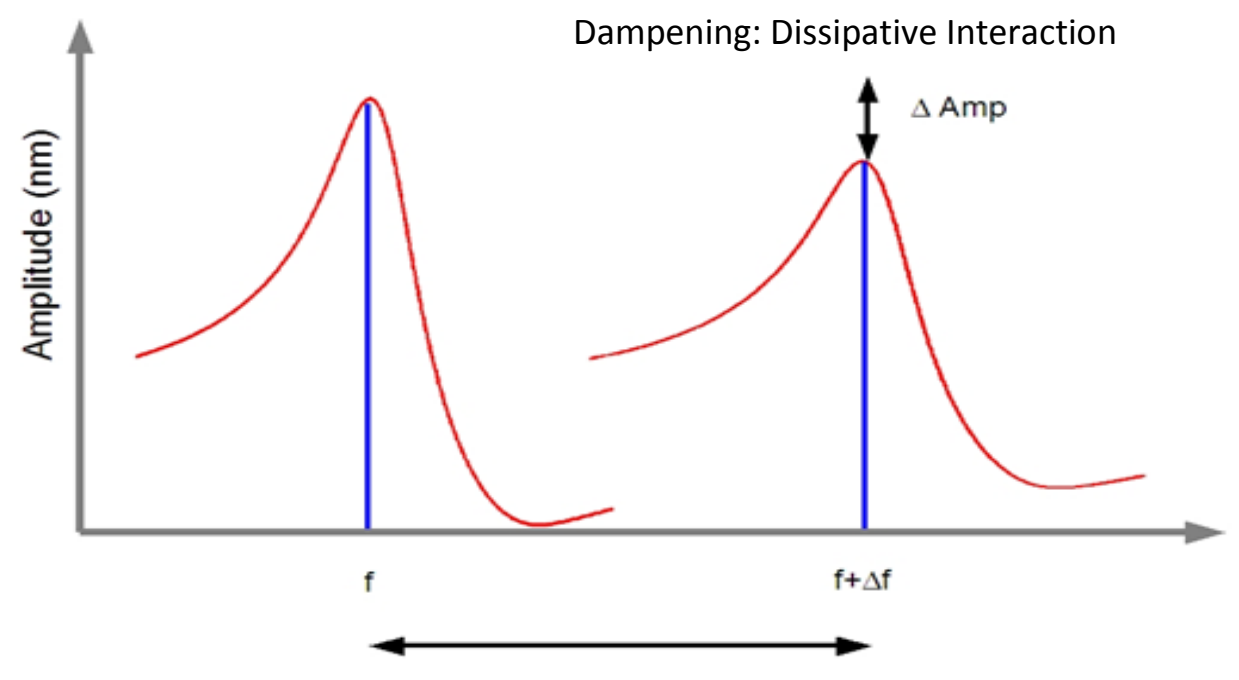

Frequency Shift: Conservative Interaction

Figure 8. Effects of conservative and dissipative forces on the spectrum of a QTF.

The faster response of frequency modulation (compared to amplitude modulation) can be key to interrogate the potential interaction forces. The time response of FM techniques is limited only by the time the sound waves travel through the whole QTF. The sound velocity sets the theoretical limit to the detection of a frequency step. 
For vertically oscillating AFM probes, the force changes within every cycle because the probe oscillates in the direction of the ARC process. As such, the average force needs to be integrated along past and future interaction positions. A significant amount of theoretical calculations has been devoted to quantitatively calculate the AFM force from the single harmonic oscillator model (SHO) [81]-[86]. For AFM cantilever probes, Giessibl[87] and Sader[88] developed equations that calculate their interaction forces. Unlike AFM cantilever probes, the QTF oscillates in the plane parallel to the surface and its average force value does not depend on the interaction along its approach curve. Thus, the single harmonic oscillator model has been successfully applied to quantitatively measure QTF interaction forces.

It is important, however, that the QTF oscillation is kept as parallel to the surface as possible (verified through optical inspection with a CCD camera). In addition, when possible, low oscillating amplitudes should be used to minimize any taping on the sample's surface.

\subsection{Equations for the calculation of interaction forces}

The calculations assume only shear interactions are being measured. We start with the driven harmonic oscillator equation with an extra term to account for the interaction forces the probe undergoes when the probe is in the layer, 


$$
m \ddot{x}+b \dot{x}+k x=F+F_{\text {int }}
$$

were $m$ is the effective mass of the system, $b$ is the damping coefficient, $k$ is the effective elastic constant or stiffness, $F$ is the driving force and $F_{\text {int }}$ is the interaction force when the probe comes into contact with the adsorbed layer as it approaches the sample's surface. Inertial drag forces are not included in this model. By making,

$$
F_{i n t}=-k_{i n t} x-b_{i n t} \dot{x}
$$

and due to the frequency modulation condition,

$$
x=x_{0} e^{-i \frac{\pi}{2}} e^{i \omega_{0} t}
$$

with,

$$
F=F_{0} e^{i \omega_{0} t}
$$

and combining equations ( 4 ) through ( 7 ), 


$$
\begin{aligned}
& k_{\text {int }}=k\left[\left(1+\frac{\Delta f}{f_{00}}\right)^{2}-1\right] \\
& b_{\text {int }}=\frac{k}{Q \omega_{00}}\left(\frac{x_{00}}{x_{0}} \frac{\omega_{00}}{\omega_{0}}-1\right)
\end{aligned}
$$

where $\omega_{00}=2 \pi f_{00}, x_{00}$ is the probe's amplitude away from any layer interaction and $x_{0}$ is the probe's amplitude within the interaction region. The amplitude of their associated forces are,

$$
\begin{gathered}
F_{k_{\text {int }}}=k_{\text {int }} x_{0} \\
F_{b_{\text {int }}}=b_{\text {int }} \omega_{0} x_{0} .
\end{gathered}
$$

These values are, of course, the amplitudes of the harmonic oscillations of the elastic force and the dissipation force experienced by the probe while inside the adsorbed layer. They will play the main roll in interpreting the results obtained in this thesis.

Using the definition of the mechanical quality factor $Q, \frac{\Delta \omega}{\omega_{00}}=\frac{\sqrt{3}}{Q}$, the driving force of the QTF is[75], 


$$
F_{D}=\sqrt{3} \frac{k x_{00}}{Q}
$$

where $Q$ is the quality factor of the QTF.

Additionally, when dealing with the friction mechanisms of the probe-sample interaction, an important parameter is the relative velocity between them. The equation for maximum probe velocity is,

$$
V_{\text {probe }}=x_{0} \omega_{0}
$$

when in resonance, which is the condition in FM-SPM.

Experiments involving a gold probe and a graphite surface under $\left(8 \times 10^{-7} \mathrm{mbar}\right.$ or $6 \times 10^{-7}$ Torr) vacuum conditions[75] have shown that expressions ( 8 ) and ( 9 ) show exponential behavior. This exponential trend leads to expressions for viscosity and shear moduli that predict values of several orders of magnitude higher than those found in bulk form.

Now we will discuss the effect of the SANM sensor in the approaching/retracting measurements. When the probe enters the adsorbed layer there exists a mechanical contact through the viscous nature of the layer. The results is a net force on the probe that translates in a restoring $\left(F_{k_{i n t}}\right)$ and drag viscous $\left(F_{b_{\text {int }}}\right)$ forces. Moreover, this viscoelastic interaction seems to occur long before the probe comes into contact with the actual solid surface (possible through displacement of the adsorbed layer). Having 
the acoustic transducer capturing the oscillations immediately beneath the sample grants an increased level of detection by determining the intensity of the interaction.

With the tip tens of nanometers away from the surface, mechanical wave fronts originating on the QTF tines' motion and traveling through air arrive to the sample. The SANM sensor detects the mechanical oscillation and its phase. The SANM amplitude provide a direct measurement of the samples vibration. Care should be taken when considering the real relationship between the surface's motion and the signal measured by the SANM. It measures the strength of the oscillation but not its direction, whether in plane, out of plane or a combination of both. The oscillation is mediated by the thickness of the substrate itself and the mechanical coupling substance (vacuum grease) which secures the acoustic transmission between sample and the SANM sensor. An analysis of the transfer mechanism is out of the scope of this thesis. However since the probe is carefully aligned for oscillations parallel to the surface, the experimental results presented herein suggest that there is a range (sometimes $6 \mathrm{~nm}$, other times $20 \mathrm{~nm}$ or higher) where the probe-sample interaction is dominated by shear forces (i.e. no hardhard touching). The information acquired is most likely related to the viscoelastic shear coupling between probe and the mesoscopic fluid-like layer.

The SANM amplitude is measured in arbitrary units and monitors the inflections and sudden changes. On the contrary, the SANM phase-signal is measured relative to the driving force phase. When the probe approaches the sample, the tip comes first into contact with the fluid-like layer and the mechanical oscillations impinged on the 
layer couple to the substrate (typically $\sim 2 \mathrm{~mm}$ thick) and transmitted to the SANM sensor located underneath (in mechanical contact with the substrate). In this approach process, when the SNR of the near field acoustic signal is high enough, the lock-in amplifier starts tracking the probe's amplitude and phase. One explanation on the origin of this phase difference is that, depending of the intensity of the elastic and viscous forces, the probe can oscillate out of phase with the QTF (to minimize this potential effect, the $100 \mu \mathrm{m}$ thick probes have been mounted such that they extend from the tines no more than $150 \mu \mathrm{m})$. Another more likely possibility[78] is the existence of a relative motion between adsorbed layer and the probe, in which case the signal from the SANM sensor would be a measure of the lagging motion of the adsorbed layer (or sample). In fact, both situations may occur one at a time or simultaneously depending on the forces involved.

The performance of a SPM system depends on its ability to sense the forces between the probe and the specimen. Tuning the parameters of a feedback control system can be implemented automatically using existing algorithms. Regardless of how good an image may look like, because of the factors discussed in the previous sections, interpreting the information contained within those images is complex matter. The vertical position control of the system may be adequate to prevent damage to the probe and obtain reproducible images but the physical dynamics involved are, very often, still subjected to proper physical interpretation. 
The forces can be classified into attractive (Van der Waals) and repulsive forces. Different theoretical models account for the repulsive, namely the Morse potential, the Stillinger-Weber potential and the Tersoff potential[31], [89]. They comprise hard sphere repulsion, Pauli-exclusion interaction and electron-electron Coulomb interaction. The repulsive forces are of very short-range and have an exponential decaying or inverse power law with high order distance dependence.

Some groups have described how changes in the elastic modulus of the sample and/or the attractive component of the force can produce variations in the cantileversurface separation, potentially leading to uncontrollable changes in the probe's height[90]-[92]. Others have concluded that contamination or salt deposits around molecules on typical support surfaces for molecules, such as mica[93], and/or dehydration could be partly responsible for height reduction[94]. It appears that the height discrepancies has contributions from the probe's geometry i.e. is an intrinsic characteristic of SPM imaging[95]. However this will not be clarified until more sensing mechanism are used to observe the same phenomena. The new acoustic techniques developed in this thesis attempt to make contributions in that direction. Their implementation is described in the next chapter. 


\section{CHAPTER 3}

\section{INTEGRATION of SHEAR-FORCE ACOUSTIC NEAR-FIELD MICROSCOPY (SANM) and WHISPERING GALLERY ACOUSTIC SENSING (WGAS) INTO a QTF-SPM}

We have integrated three techniques in one single metrology tool to address two main issues. The first is to determine the distance at which the mechanical coupling between probe and sample begins. In other words, the conditions of distance and force where the sample (a mesoscopic fluid adsorbed on a solid surface) starts to reveal elastic response to the perturbing probe. This second goal is to attempt to determine whether the mechanical response of the liquid diverges from its bulk properties. Such challenges may require not only the measurements of forces with high sensitivity and, ideally, sub-nm resolution, but also different techniques (sensing different physical properties) working in tandem complementing their capabilities. Our answer to those challenges has been bringing together WGAS, SANM and STM into a single metrology system.

Performing the experiments at ambient conditions, makes the theoretical modeling significantly more difficult due to the striking properties, quite different from the bulk, of the adsorbant molecules. The new concepts in the literature, such as nanoindentation, nanoconfinement, nanowear and nanoheat, reflect the diversity of fields coming together to tackle this difficult subject. Although different models have been introduced to describe interactions between the SPM-probe and the sample- 
substrate, the most successful ones are those that make prediction for experiments performed under high vacuum conditions. The presence of the liquid trapped in between the solid surface appears to complicate the scenario very much. In fact, a full understanding of the physical properties of mesoscopic fluids under confinement between two solid boundaries remains a major challenge in condensed matter physics.

We hope the new acoustic sensing mechanisms described in this thesis could open new paths for analysis thus contribute to a further understanding the dynamic behavior of confined mesoscopic fluids. The next sections of this chapter describe in more detail the integration of WGAS, SANM and STM into a single metrology system.

\subsection{Experimental setup}

Figure 9 shows a schematic of the experimental arrangement that uses a WGAS to monitor the probe's lateral oscillation amplitude, SANM, to monitor the mesoscopic fluid response, and the optional monitoring of the tunneling current (when testing conductive samples with conductive probes). It operates as a regular QTF-SPM, the QTF being driven at its resonance frequency, but the probes motion is monitored by WGAS instead of the electrical response from the QTF (see Figure 5 for comparison). In addition to the simplicity of its implementation, the WGAS provides two main advantages: a) Direct correlation with the mechanical response of the QTF (which is not possible when the signal is measured electrically) and b) The resonant cavity can be designed to have resonant modes coinciding with the resonant frequency of the QTF 
(thus increasing the SNR of the system) which would put WGAS in better grounds to compete with the current state of the art QTF-SPM system.

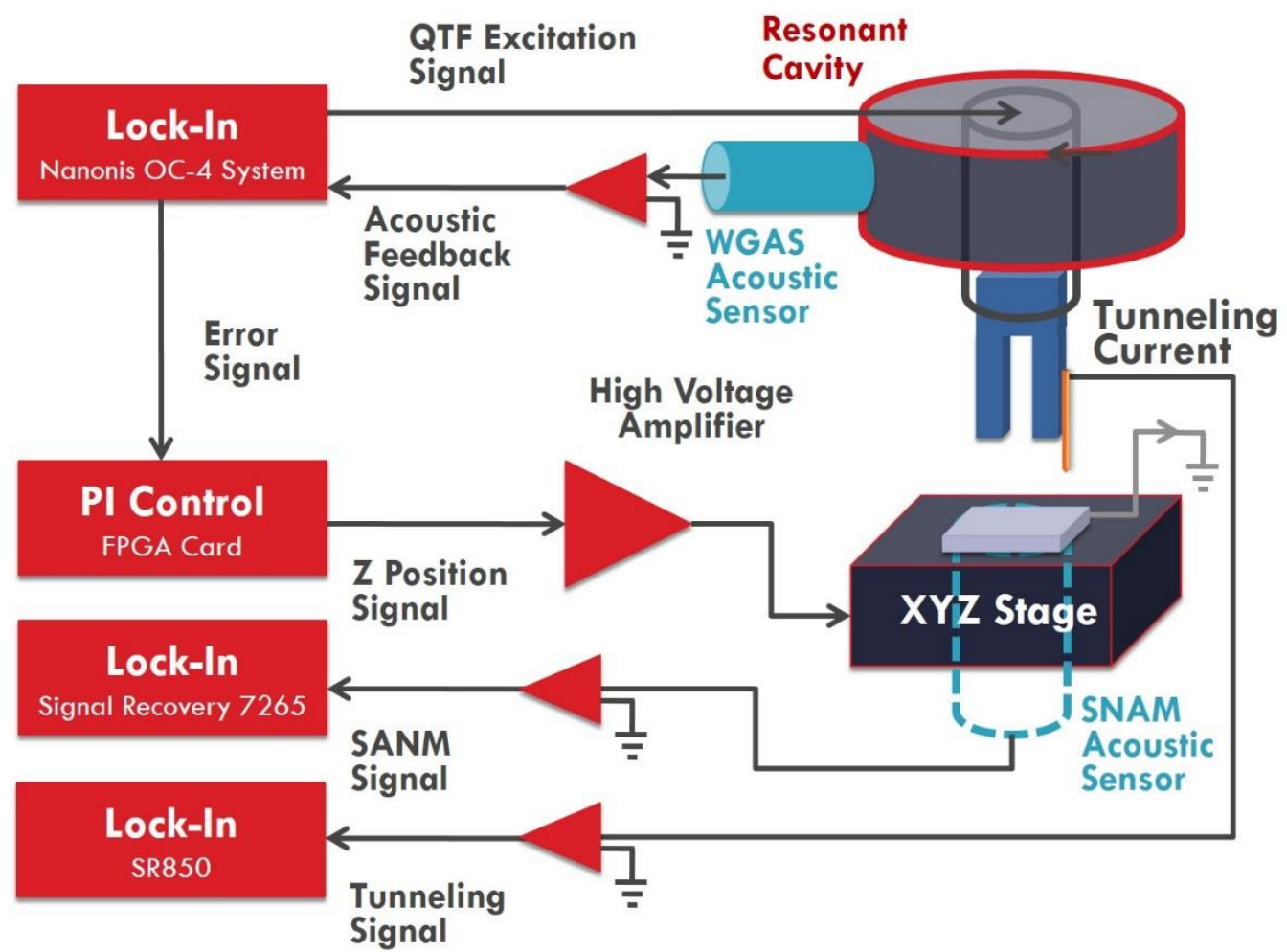

Figure 9. Diagram of the WGAS and SANM integration into a QTF-SPM. The monitoring of the tunneling current is optional (when testing conductive samples); in that case the ac-voltage driving the QTF is used to bias the metallic probe.

During the probe sample interaction, part of the energy (in acoustic form) is transmitted through the sample; the purpose of the SANM is to detect that energy by placing a sensor beneath the inspected specimen. 
The WGAS sensor is placed on top of the cavity with an applied film of vacuum

grease between them, to maximize mechanical coupling, and held in place by a aluminum plate with adjusting screws coupled with springs that maintain a constant force on the back of the acoustic sensor. In the next section we describe the parts of the SANM-WGAS integrated SPM system.

\subsection{Components overview}

The SANM-WGAS is an aggregate of several commercial and costum-made components, adapted to fit the requirements of the ARCs process. The main parts of the system are,

- The OC-4 PLL Nanonis system

- $\quad$ FPGA card (PCle bus)

- FPGA breakout box

- Nano-Z50HS piezo stage with driver controller

- Approaching and retracting software control

- Set of piezo-mechanical sensors

- Set of preamplifiers and analog filter stages

- Set of custom made cables

We will briefly describe each part in the next sections. 


\subsection{A The OC-4 PLL Nanonis system}

The OC4-PLL transforms the Quartz Tuning Fork (QTF) signal from voltage to values of amplitude, phase and frequency shift. The last two are determined with respect to the sensor's excitation signal produced by the same OC-4 PLL. It communicates with every other portion of the control system through its analog inputs and outputs fitted with BNC connectors. The datasheet for the OC-4 can be found at http://www.specszurich.com/upload/cms/user/OC4ProductBrochure.pdf. We will briefly describe the main inputs and outputs and how they relate to the rest of the system.

There are two connector groups visible separated. The group on the left consists of connectors related to the QTF's excitation and measured signal.

i. Inputs. The PLL has two BNC inputs on its front panel. The first is labeled 'INPUT' and receives the signal from the QTF. The second input is labeled 'ADD' and can be used to provide a second excitation signal, at a different frequency than the produced by the OC-4. The signal entering this input will be added to the excitation output.

ii. Outputs. The PLL outputs are the amplitude, phase, frequency shift and two auxiliary outputs. There are two additional output that will not be explained here and are not relevant to our process. It is worth noting that the 'FREQ' output is the difference between the 'center frequency' (set by the user), the 'PHASE' is the phase between the excitation and measured signal, and the 'EXC' signal is 
the amplitude of the OUTPUT connector (excitation). The AUX 1 can be set to amplitude, frequency or phase, multiplied by a constant or offset by a constant. Figure 10 shows the front panel of the OC-4.

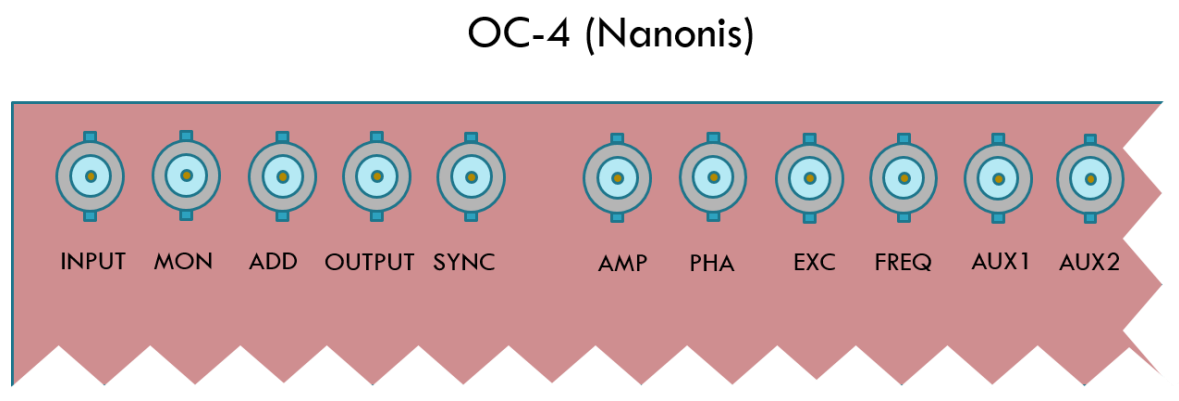

Figure 10. Front panel of the OC-4 PLL stage. The outputs to the right are not shown.

\subsection{B The FPGA card}

The FPGA is the acronym for the Field-Programmable Gate Array. It is an arrangement of digital gates (NAND's, NOR's) that can be modified by applying electric fields, creating new circuits without physically changing the traces in the circuit. This provides a flexible environment for creating circuits to perform tasks that would take too much time for a PC to perform. As an example consider the approximately 2 ms latency, which is the time between a command issued and the task performed, of a normal PC running Windows. In addition, the latency is not constant and the variation depends on the particular number of tasks the operating system is occupied with, and its priorities. Naturally, a PID control needs the highest priority and a real-time response when trying 
to control probe and sample, a few nanometers away from each other. Morever, the FPGA can process operations as fast as every 45 nanoseconds.

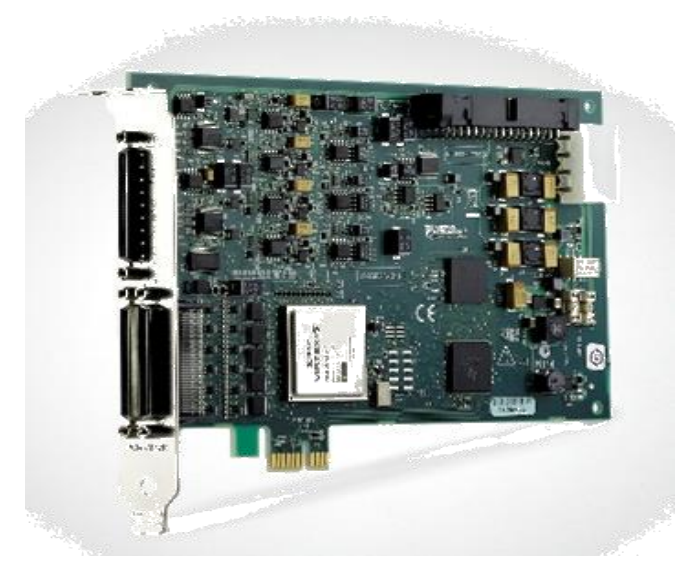

Figure 11. FPGA card from National Instruments (NI). http://www.ni.com/fpga/

The response time of the FPGA, however, is limited by the time it takes the analog to digital converter (ADC) to convert the analog signal to a digital signal and vice versa. With a rate of 750 ksamples/second at the input and 1 Msamples/second at the output, the current time for the PCle-7852R card is (the inverse of the figures above) $1.33+1=2.33 \mu \mathrm{s}$, which is appropriate for the purpose of controlling the tip-sampledistance. As a National Instruments (NI) product, the PCle-7852R card is programmed with LabVIEW. The FPGA contains the PID algorithm, performs the initial data processing, filtering of the acquired signals, etc. 
The most time-sensible calculations are sent to the PC using direct memory allocation (DMA) transfer and the rest are sent (or received) using graphic user interface (GUI) run on the PC's processor.

\subsection{The FPGA breakout box.}

The FPGA breakout box is connected to the actual FPGA, which is located inside the PC through a special National Instruments (NI) cable. The purpose of this box is to provide easy communication between the FPGA card and the external world through connectors with easy access. It has 8 BNC connectors (with the option to add more, the only restriction is the geometry of the box) for analog and digital inputs and outputs (I/O). In essence the breakout box is the front panel of the FPGA. All of the signals converge here and all of the command signals (the signals that drive the motion of the piezo-stages) are delivered from it.

\subsection{The high resolution stage (HRS)}

The high resolution stage (HRS) increases the voltage resolution by adding two

signals: a first signal (named 'fine') is divided over a certain factor (100 in our case) and is added through a summing amplifier to the second signal (named 'coarse'). 


\section{FPGA Breakout box}

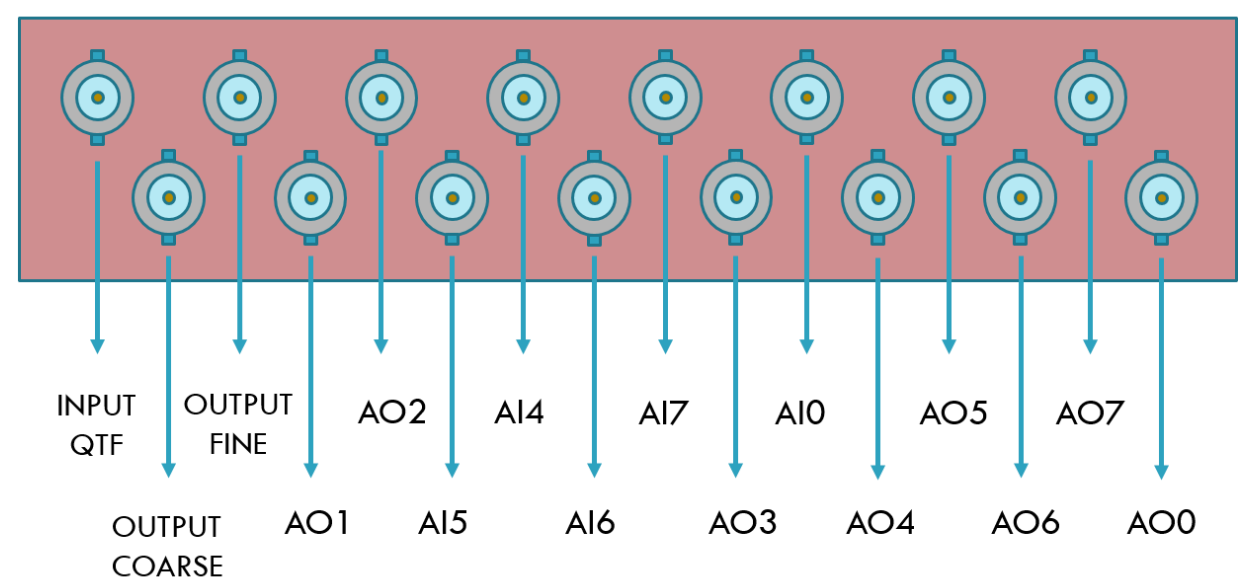

Figure 12. Front panel of the FPGA breakout box. The connectors shown (apart from input, output fine and output coarse) can be exchanged internally using FPGA programming.

In this way the resolution is improved 100 times, which would reduce its total range by the same factor used to divided the 'fine' signal, but the second 'coarse' signal offsets the final output by synchronously shifting to a value equal to the full range of the maximum range of the 'fine' effectively compensating the reduction in total voltage range. A more detailed explanation will be offered later in the chapter. Figure 13 shows the connections for the HRS. 


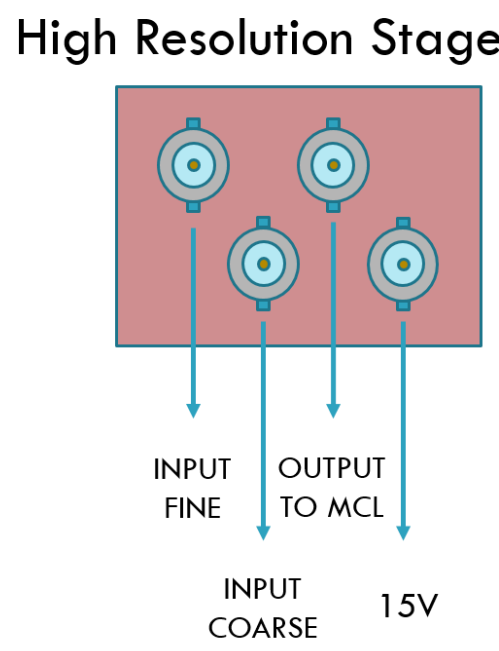

Figure 13. High resolution stage (HRS) front panel.

\subsection{E The pre-amplifiers}

The SANM, WGAS and tunneling signals need amplification stages before they reach the lock-in inputs, as shown in Figure 9. The SANM sensor has its own commercial amplifier stage, (module 500J from DECl) and the WGAS uses the custom-made trans-impedance amplifier described in more detail in section 3.3.A. The tunneling signal uses the SR 570 low noise current preamplifier from Stanford Research Systems for its amplification before it reaches the SR-850 lock-in amplifier, also from Stanford Research Systems. 


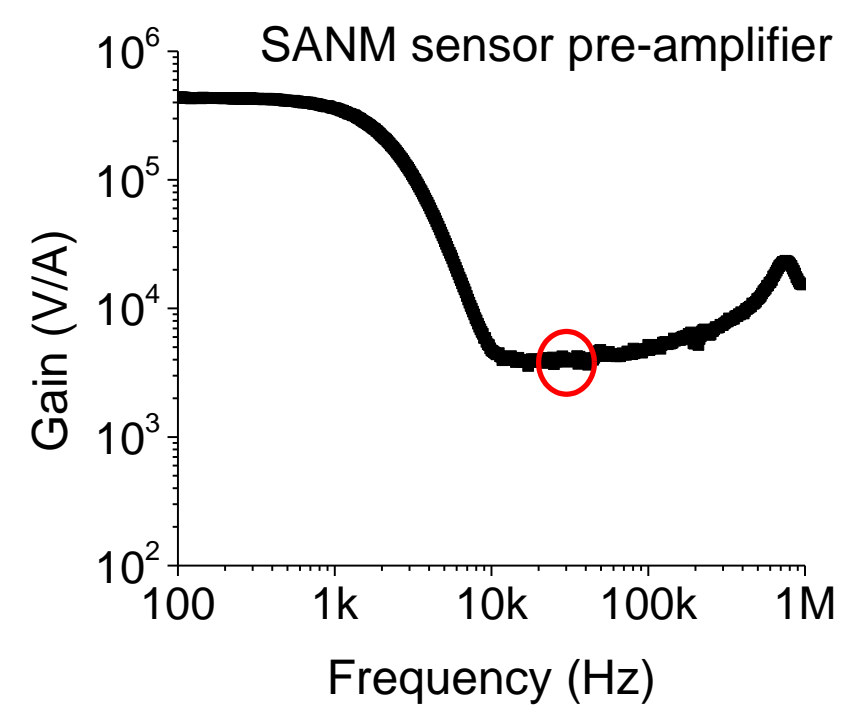

Figure 14. Gain of the preamplifier of the SANM sensor. The gain at $32 \mathrm{kHz}$ (indicated by the circle) is $3950 \mathrm{~V} / \mathrm{A}$.

\subsection{F Custom-made cables for mechanical decoupling}

One of the early challenges was the mechanical coupling to the base of the SANM-WGAS system through the electrical cable of the SANM sensor, since it is attached to the Nano-Z50HS piezo stage (serving as the sample holder). The mechanical coupling was minimized by using a ultra-flexible shielded cables (Brim Electronics, product number 1210/5, http://brimelectronics.com/1208-1210.htm) to fabricate the SANM acoustic sensor connections. This shielded flexible cable minimizes any mechanical vibrations and strains placed on the SANM sensor by the sensing cables that could damage the Nano-Z5OHS platform. 


\subsection{G Piezo-acoustic sensors}

Acoustic sensors from Dunegan Engineering Company Incorporated (DECl) were used. The WGAS utilizes the SE25-P acoustic sensor and the SANM uses the SE40-Q. Both have their highest sensitivity around $32 \mathrm{kHz}$.

\subsection{H The Nano-Z50HS stage}

The Nano-Z50HS is a high speed ( $1 \mathrm{kHz}$ resonance frequency) uniaxial piezo stage from Mad City Labs. In this thesis the piezo was used to calibrate the displacement of the piezotube used for the ARCs. It has closed loop control of its position and a $0.1 \mathrm{~nm}$ resolution.

\subsection{Signal Conditioning}

Minimizing the noise level is very important in electronic measurements. A typical procedure to evaluate the noise of a system is to break it into contribution from different component.

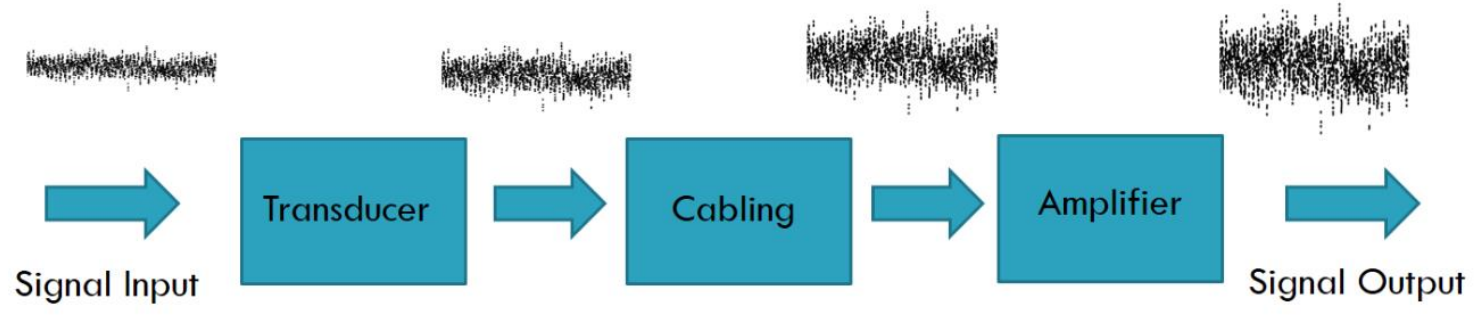

Figure 15. Schematic of the noise addition. Each stage introduces noise to final signal output 
When having a series of stages where the output of each stage is connected directly to the input of the next one, each one of the stages contributes to the final noise level. This contributions add in quadrature,

$$
V_{\text {total noise }}=\sqrt{V_{\text {stage } 1}^{2}+V_{\text {stage } 2}^{2}+\cdots}
$$

To assure a clean control signal a series of filters must be included for each measuring channel. For data acquisition this is done with the help of the FPGA card PCle 7852R and LabVIEW digital filter packages, where each signal has a real time digital filter of $4^{\text {th }}$ order, low pass Butterworth and $15 \mathrm{~Hz}$ to $50 \mathrm{~Hz}$ cut off frequency, depending on the particular experiment. For the driving (controlling) signals, however, the filter (amplifier) must be implemented right before (right after) the transducer, since the PZT driving signal (QTF/WGAS signal) requires a clean driving voltage (high gain amplification).

\subsection{A Trans-impedance amplifier}

The trans-impedance amplifier is a particular case of the inverting operational amplifier setup. The main two parameters that are important in this study are the noise level and the gain in a frequency range at around $32 \mathrm{kHz}$. An important equation impacting the performance of the amplifier is,

$$
f_{\text {cut off }}=\sqrt{\frac{\text { Gain Bandwidth Product }}{2 \pi R C_{i}}} .
$$

Figure 16 shows the diagram and a drawing of the trans-impedance amplifier implementation. 

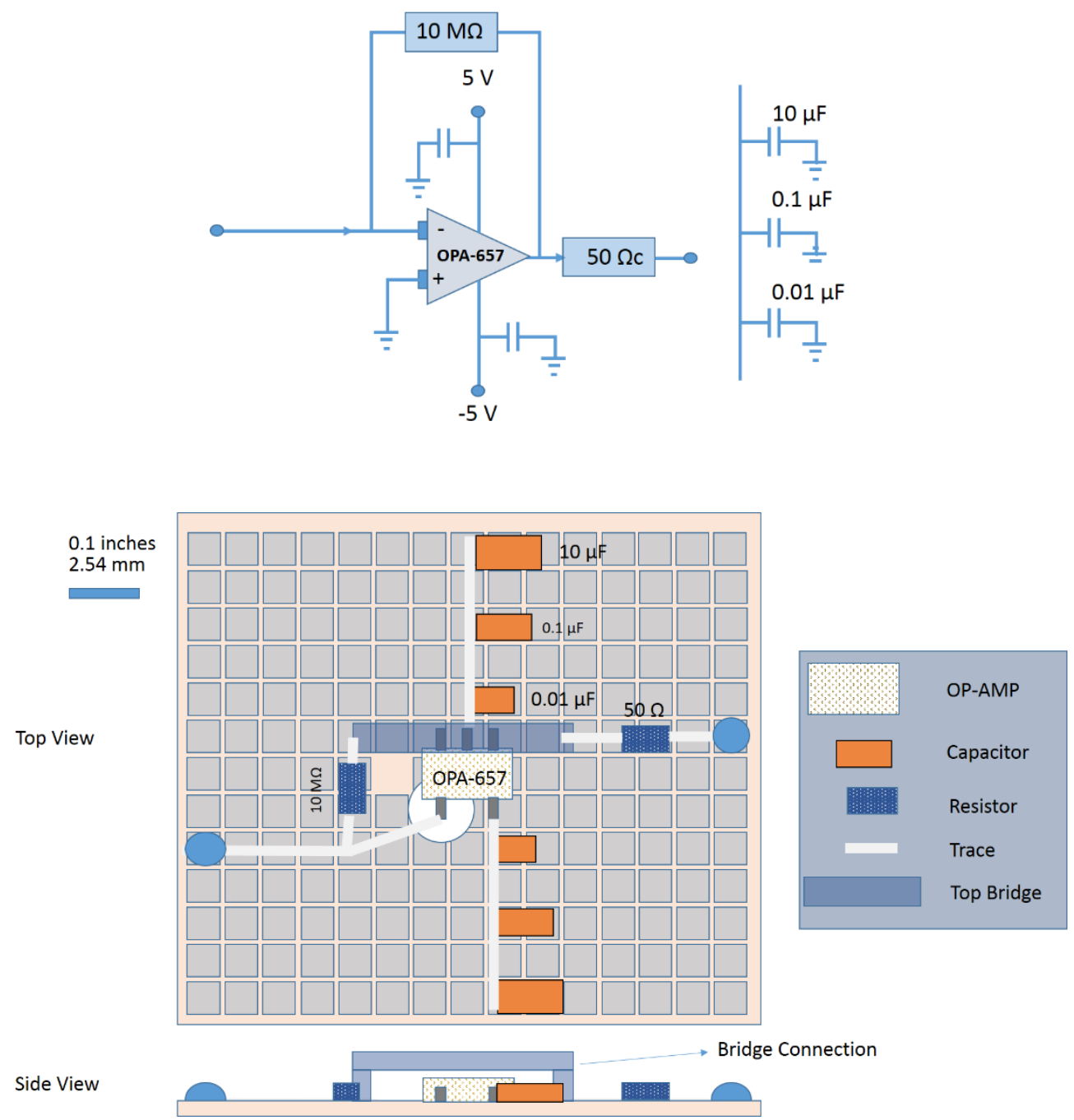

Figure 16. Top: Trans-impedance amplifier circuit diagram. Bottom: Board implementation.

The equation emphasizes the role of the input capacitance $C_{i}$. The larger its value the smaller the bandwidth. A thorough search resulted in choosing the OPA 657 due to its high Gain Bandwidth Product (GBP), low input voltage and low current noise[96], [97]. 


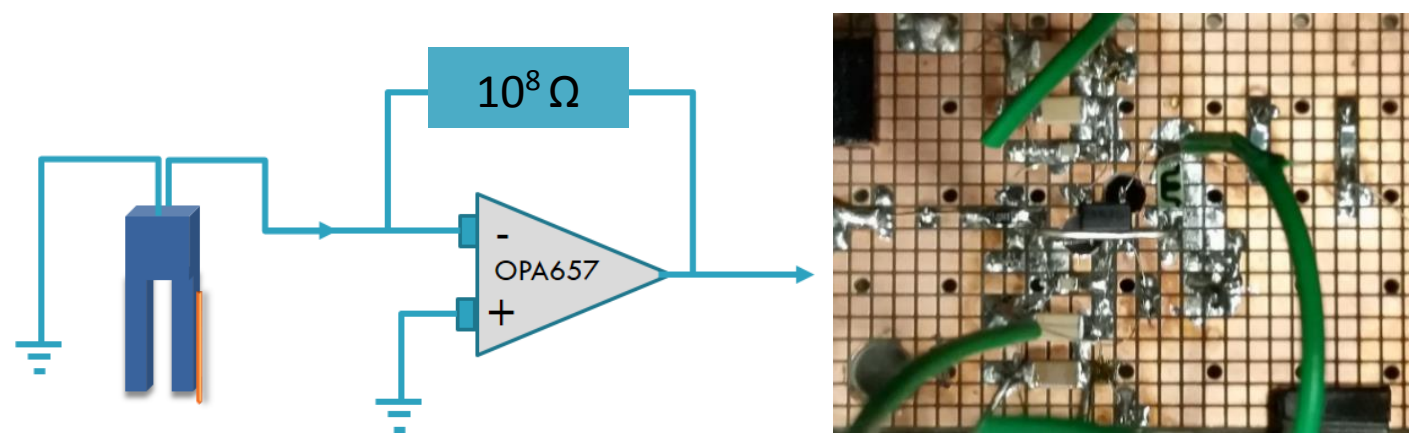

Figure 17. Left: Diagram of the QTF and trans-impedance amplifier. Right: Actual transimpedance amplifier circuit inside its enclosure displaying its assembled surface mount components.
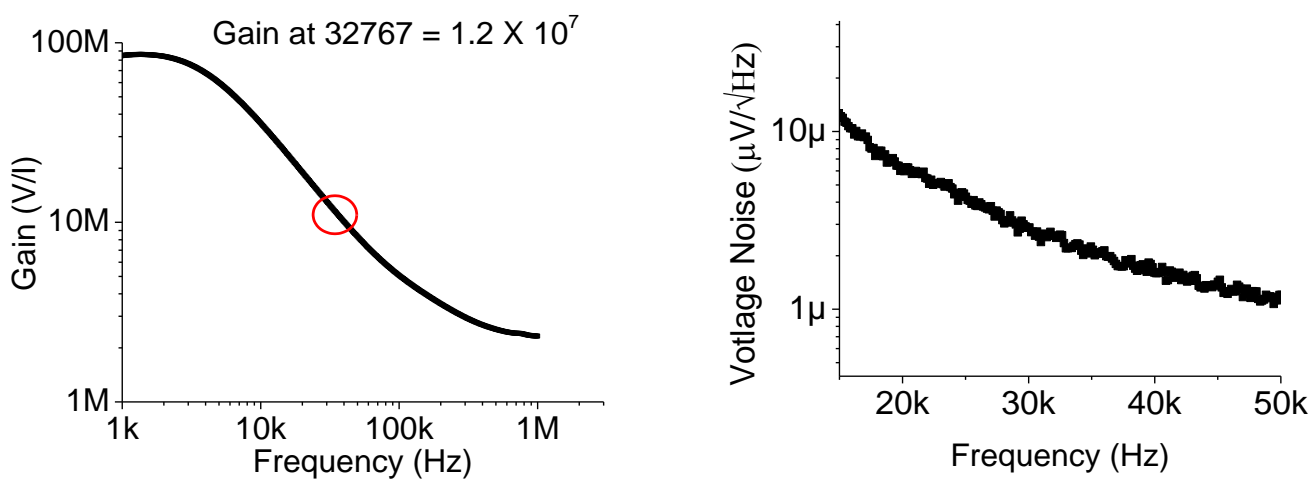

Figure 18. Gain response of the QTF/WGAS-preamplifier (left) and its corresponding noise spectral density (right).

\subsection{B Active Filtering.}

For frequencies above $1 \mathrm{MHz}$ filters are usually implemented with passive components, namely resistors, inductor and capacitors. However, for $\mathrm{f}<1 \mathrm{MHz}$ the relevant inductance components need larger more expensive values, which coupled with the bigger size make them less attractive. As an alternative, active filters (which use 
operational amplifiers) provide similar characteristics in this frequency range[98]. As opposed to passive filters, active filters do not need impedance matching techniques since the output impedance of the circuit is the low value associated with op-amp. The cut off frequency can be tailored using the proper resistor and capacitor values. In addition, using surface mount components help reduce the electric path significantly.

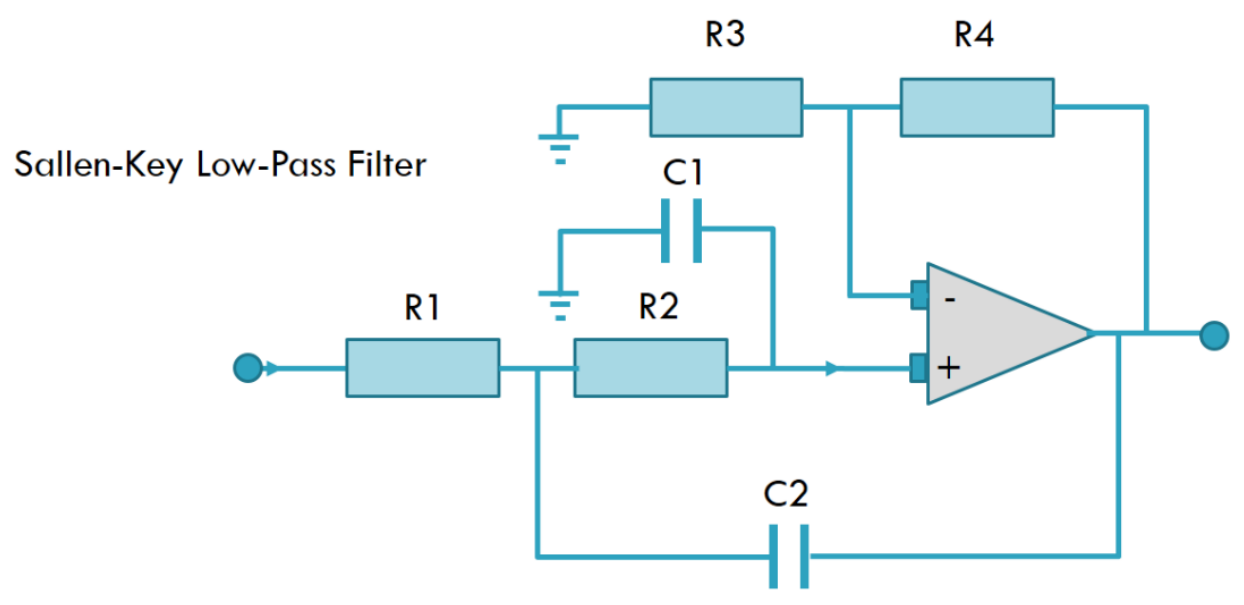

Figure 19. Active low pass filter of Sallen-Key topology. This design only needs one operational amplifier per filter at the expense of smaller roll off[98].

The cut off frequency for the PZT low pass filter was set close to $30 \mathrm{~Hz}$; using lower values to would be incompatible for feedback purposes with an error signal of 130 ms time constant.

\subsection{Software development in LabVIEW}

Feedback control in typical SPM systems with normal speed operations (1-5 line/s) requires fast responses in the order of tens or hundreds of microseconds. Since 
the Proportional Integral Derivative (PID) control loop needs to respond to interactions in the order of sub-milliseconds, PID loops running in a Windows environment, which have a latency of a few milliseconds, are inadequate. An FPGA is a reprogrammable integrated circuit that can achieve high processing speed (of tens of nanoseconds) utilizing the resources of the operating system running the personal computer (PC) the card is installed in. In this thesis PCle-7852R FPGA from National Instruments was used.

The card contains a Virtex-5 LX50 FPGA onboard chip whose functionality can easily be changed using the LabVIEW software package; eight independent 16-bit analog inputs with $750 \mathrm{kHz}$ update rate; eight independent 16-bit analog outputs with bandwidths of $1 \mathrm{MHz}$; and 96 digital lines at a rate of $40 \mathrm{MHz}$. The parallelism of the FPGA enables all of the analog I/O and digital I/O ports to be accessed simultaneously.

FPGA programming in LabVIEW has two distinct environments, the "PC" and the "Target" environment. The algorithm written for the PC environment is executed using the PC's microprocessor and is in charge of the graphical user interface (GUI) and of relatively low speed calculations. A second algorithm written in the "Target" environment is loaded onto the FPGA, capitalizing on the resources available in the card and able to communicate with the PC environment program.

For the implementation of the PID program, Direct Memory Access (DMA) data transfer and synchronization techniques were included to improve the efficiency of the data transfer rate between the FPGA and the PC (lightening the work of the PC 
processor). A single FPGA card enabled the system to acquire at least 6 different channels of information.

\subsection{A Modified Proportional Integral Differential (PID) Feedback for High Resolution Control}

The modified PID used in this thesis is a complex adaptation of the PID FPGA virtual instrument (VI) found in the LabVIEW library. It has gone under extensive modification to accommodate for the high resolution output (HRO) implementation (Figure 20). The HRO generates two signals, the PID output signal and the Offset signal. The PID output $(v 2)$ behaves as a regular PID signal, with the difference that when it reaches its maximum (minimum) value it returns to its minimum (maximum), depending on the direction of change (Figure 20). As this transition occurs, the offset signal (v2) changes its value by $\beta \times 20$ Volts (in this thesis $\beta=1 / 100$ ) and since the PID output signal will later be multiplied by the same factor, when both signals are added the resulting signal (high resolution PID in Figure 20) will be a continuous monotonic curve. The addition of the two signals is performed by the summing amplifier also shown in Figure 20.

\subsection{B Approaching and retracting control program (ARCP)}

The development of the approach and retraction program (ACRP) responded to the need for increased repeatability and temporal accuracy of the ARCs. The SPM used 
in this thesis was initially designed for the imaging of biological samples, requiring precision range in the nanometers.
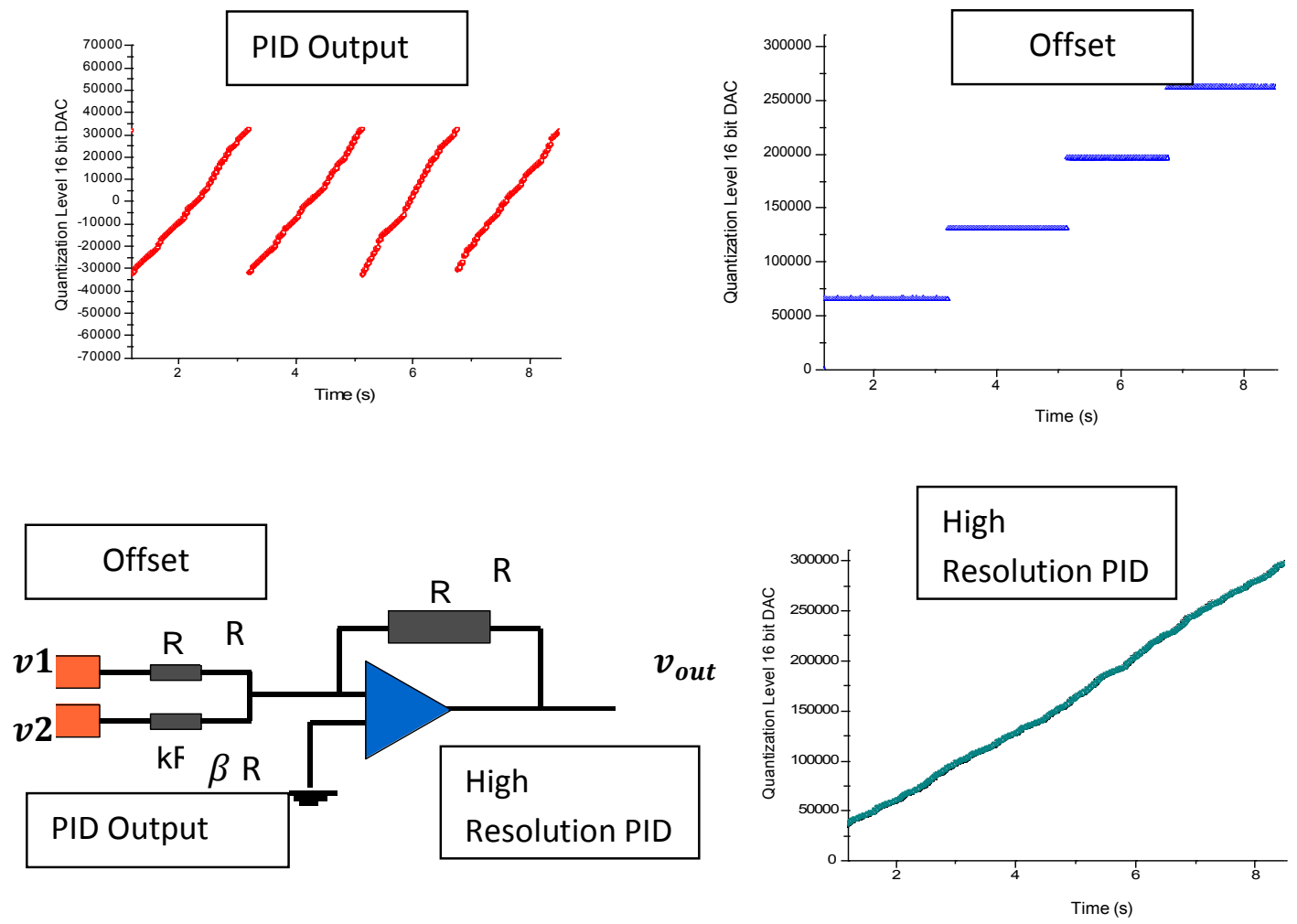

Figure 20. High resolution output (HRO). Two 16 bit signals are synchronized to produce a continuous higher resolution (100 times higher in this thesis) than each channel could deliver separately. The resulting output value is $v_{\text {out }}=v \mathbf{v} \times \frac{R}{R}+\boldsymbol{v} \mathbf{2} \times \beta \times \frac{R}{R}=\boldsymbol{v} \mathbf{1}+\boldsymbol{v} \mathbf{2} \times \beta$.

However, when the influence of mesoscopic fluid film entered into the NSOM scenario, as a potential factor that could influence the high-resolution images, the investigation of the adsorbed mesoscopic layer require sub-nanometer precision since the first monolayers adjacent to the solid boundary could be the most influential ones in 
the dynamics of the fluid layer. The thickness itself of the layer could be as small as 1.5 $\mathrm{nm}$ as occurs in the case of a freshly peeled mica sample[99]. One important factor to take into account in this endeavor to attain nanometer precision position control is the unavoidable thermal drift of the SPM stage. Accordingly, the ARCP program has incorporated a protocol that performs an evaluation of the thermal drift prior to recording an approaching/retraction curve, so a proper correction can be applied afterwards:

i. Surface reference seeking. The systems sets the probe's oscillation amplitude to a pre-determined value. As the probe moves vertically towards the sample the system control waits until the oscillation amplitude decreases to a predetermined setpoint value, which triggers a retraction (see next step). The maximum approaching speed is $50 \mathrm{~nm} / \mathrm{s}$, but we typically use $5 \mathrm{~nm} / \mathrm{s}$. At this point the system knows where the air-fluid interface is located.

ii. Backing-out. After the control signal reaches the setpoint, the system retracts a distance (usually set at $40 \mathrm{~nm}$ ) to keep the probe hovering just above the interaction region. It is here where the option of drift control is chosen. If the user selects the 'drift control' option the software starts that process (explained in the next chapter). If not it continues with the third step.

iii. Drift correction. The probe changes to feedback mode and approaches the surface to maintain a constant interaction force (given by setpoint of the control signal). The details of the drift correction process will be described in chapter 4 . 
iv. Approaching curve. The approach starts at a predetermined velocity

v. Retracting curve. The retraction starts at a predetermined velocity.

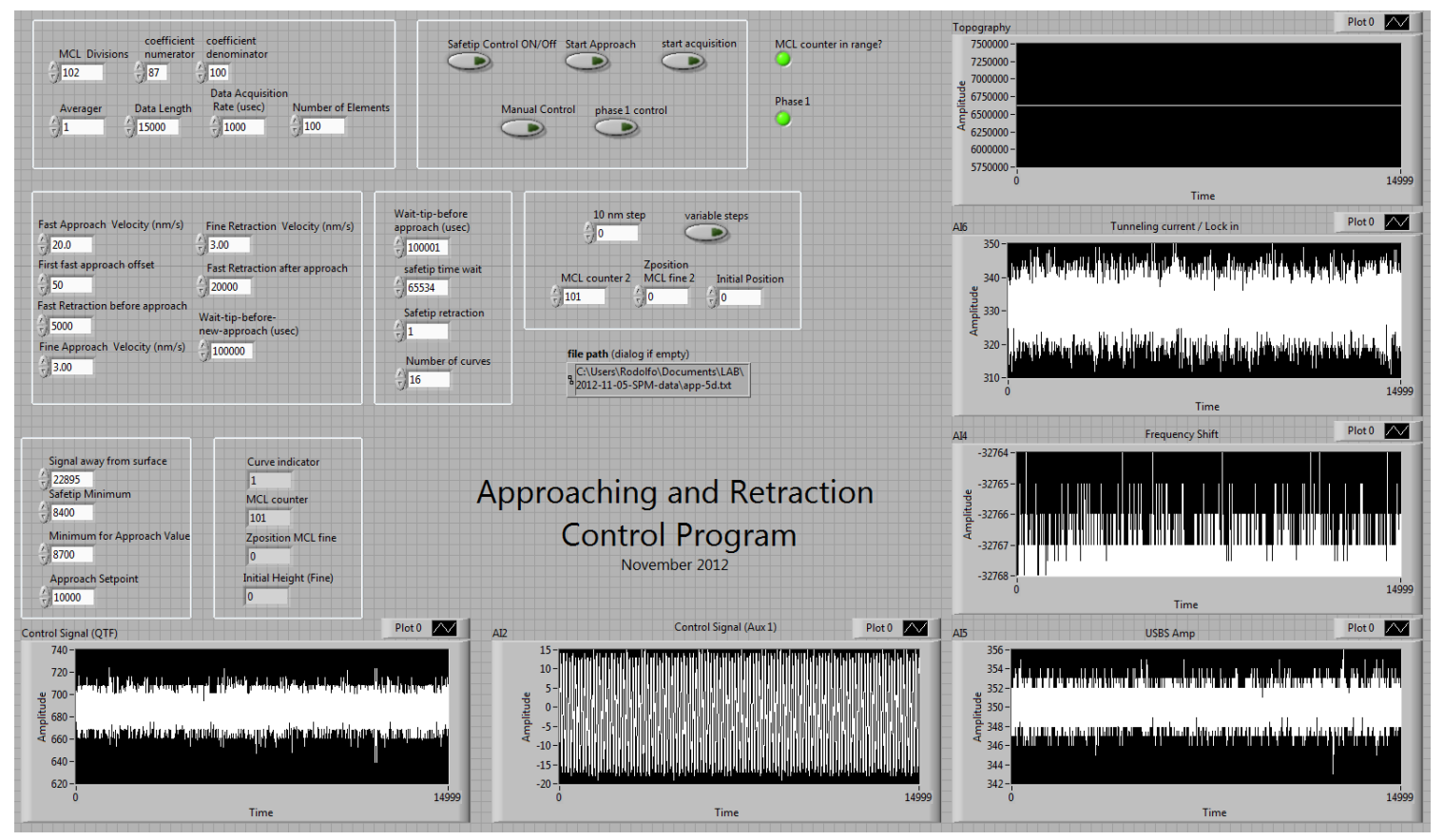

Figure 21. Approaching and Retracting Curve Program (ARCP) developed to control and perform drift corrections on every approaching and retracting curve (ARC).

Figure 21 shows the front panel of the ARCP. The ARCP allows performing ARC's curves continuously and with high reproducibility. When necessary it can also execute manual control of the distance between the probe and the sample.

\subsection{Determination of the amplitude of oscillation of the QTF}

The measurement of the noise floor of the trans-impedance amplifier determines the sensitivity or minimum distinguishable value of the physical variable 
used as the control signal. In the case of the QTF, this will determine the sensitivity of the control signal and the force measurements. We use the high gain preamplifier discussed above, an acquisition time of 60 minutes with steps of $15 \mathrm{mHz}$ and a time constant of $52 \mathrm{~ms}$.

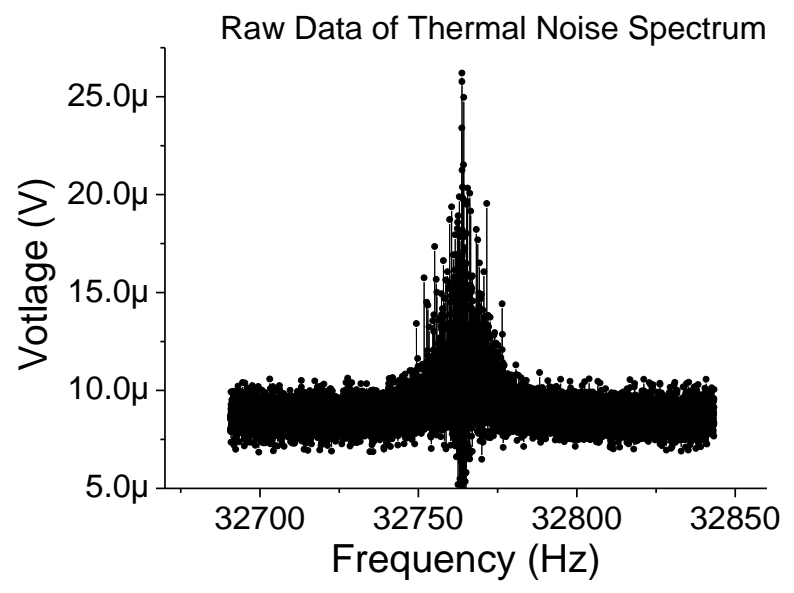

Figure 22. Raw data of the QTF spectrum. The QTF was kept in its vacuum metal container and soldered right next to the input of the board of the pre-amplifier. One of the tines of the QTF is grounded and the other connected to the input of the pre-amplifier.

The QTF response from Figure 23 is caused by the Johnson-Nyquist thermal voltage noise of its equivalent resistance $R$ of the Butterworth-Van Dyke[70] model. It is measured by grounding one of the excitation electrodes and connecting the other to the input of the preamplifier, shown in Figure 24. 


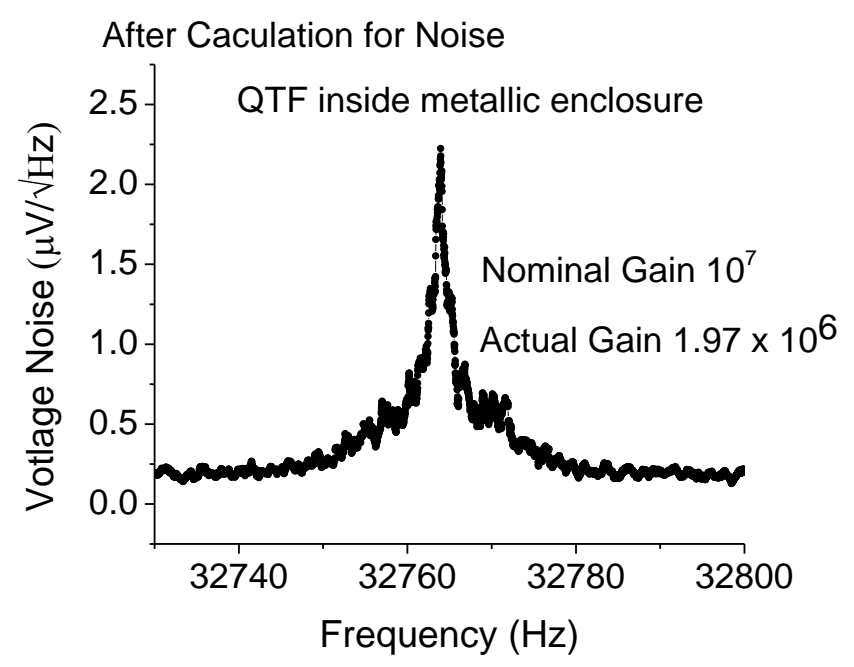

Figure 23. Noise density spectrum of the QTF obtained by calculating the standard deviation of the average signal from the spectrum in Figure 22 above. The algorithm used had an averaging window of 100 points equivalent to a $1.5 \mathrm{~Hz}$ range.

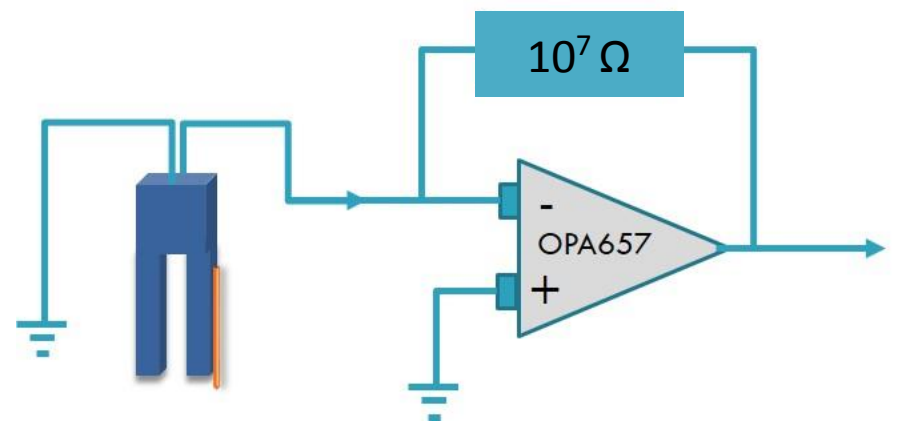

Figure 24. Schematic connections to measure the QTF's response due to thermal noise.

However, this conditions do not resemble the ones in while the approaching and retracting curves (ARC) are performed. Furthermore, calibrating the QTF in this way would require opening and rewiring the boards input connections, soldering the QTF 
directly at the op-amps input every time a new probe is used. To more closely reflect the actual conditions and to develop a more practical approach to the QTF's calibration, the QTF was placed outside the pre-amplifier box, although still inside a metal box, and its spectrum taken.

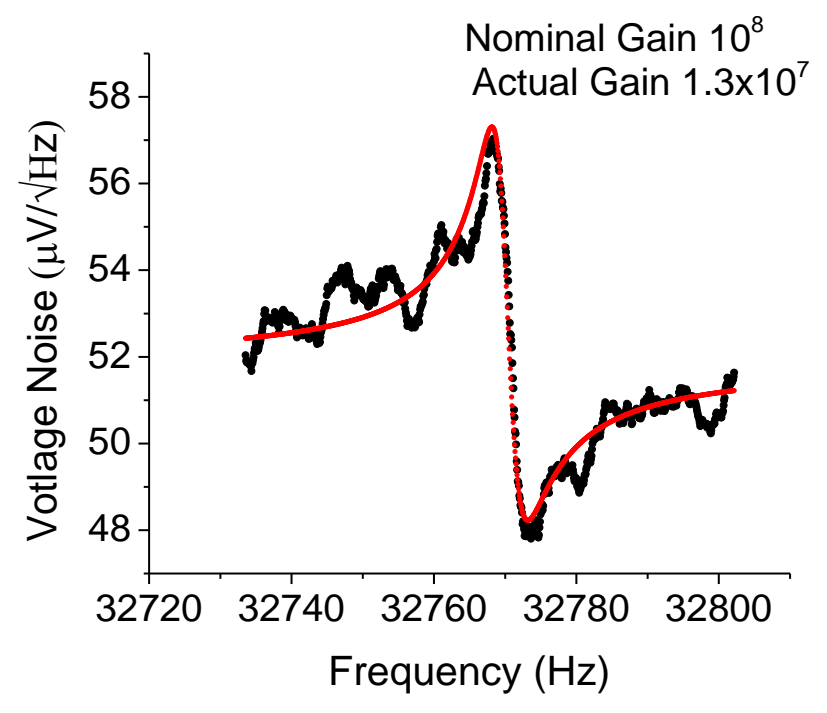

Figure 25. Thermal noise spectrum and fitting curve of a QTF still enclosed in its metal cylinder but connected to the amplifier with a 20 $\mathrm{cm}$ of coaxial cable. The capacitance of the connections increase the asymmetry of the spectrum.

This time the capacitance increased due to the cabling and extra connections between the QTF and the input of the circuit board. The result was the well-known asymmetry around the resonance frequency[100]. 
By fitting the spectrum to the expression,

$$
I(\omega)=\frac{I_{0} \omega}{Q \omega_{0}} \sqrt{\frac{1+2 \frac{C_{0}}{C}\left(1-\frac{\omega^{2}}{\omega_{0}^{2}}\right)+\frac{C_{0}{ }^{2}}{C^{2}}\left(1-\frac{\omega^{2}}{\omega_{0}{ }^{2}}\right)^{2}+\frac{C_{0}{ }^{2}}{C^{2}}\left(\frac{\omega}{\omega_{0} Q}\right)^{2}}{\left(1-\frac{\omega^{2}}{\omega_{0}{ }^{2}}\right)^{2}+\left(\frac{\omega}{\omega_{0} Q}\right)^{2}}}
$$

we can extract the equivalent RLC parameters[100] using the additional relations $\omega_{0}=$ $1 / \sqrt{L C}, Q=L \omega_{0} / R$ and $I_{0}=V / R$. To calculate $C$ we express it in terms of $R, Q$ and $\omega_{0}$ using these relations. After algebraic reordering we obtain,

$$
C=\frac{1}{R Q \omega_{0}}
$$

Additionally, since the excitation is thermal in origin, the expression is given by,

$$
\overline{v^{2}}=4 k_{B} T R
$$

in units of $V^{2} / \mathrm{Hz}$. The expression for the $\mathrm{R}$ is,

$$
R=\frac{4 k_{B} T \Delta H z}{\left(I_{0_{R M S}}\right)}
$$

and the results is $\mathrm{R}=2.44 \times 10^{5} \Omega$. We use this value, a bandwidth $\Delta H z=5 \mathrm{~Hz}$ and the fitted parameters to obtain $C=2.83 \times 10^{-15} \mathrm{~F}\left(C_{0}=8452.7 \times 2.83 \times 10^{-15} \mathrm{~F}=23.4 \times 10^{-12} \mathrm{~F}\right)$.

The value of $k$ is calculated from equation ( 3 ) using the experimental values $t=0.55$ $\mathrm{mm}, \mathrm{L}=3.75 \mathrm{~mm}$ and $\mathrm{w}=0.25 \mathrm{~mm}$. The result is $15518 \mathrm{~N} / \mathrm{m}$. Using equation $(1) \alpha=$ 
$\sqrt{\frac{k C}{2}}$ we find a value of $\alpha=4.69 \times 10^{-6} \mathrm{C} / \mathrm{m}$ which in turn delivers a value of $1.93 \mathrm{~A} / \mathrm{m}$ for $\Delta I_{R M S} / \Delta x_{R M S}$ using equation ( 2 ), $\Delta I_{R M S}=4 \pi f \alpha \Delta x_{R M S}$. To corroborate the above calculations we took the calculated $\alpha$ and use it to calculate $k$ again with the application of the equipartition theory to the analysis of the QTF motion[101]. The known relationship is

$$
k=\frac{k_{B} T}{\left\langle A_{\text {thermal }}^{2}\right\rangle} .
$$

We obtain a $k$ of $6331 \mathrm{~N} / \mathrm{m}$, half of the value obtained above.

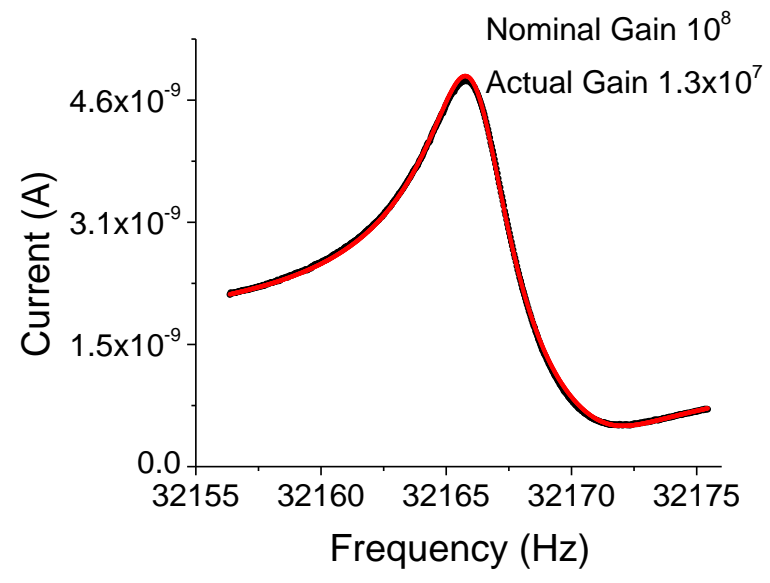

Figure 26. Spectrum taken to an actual QTF right before the approach and retraction process, hovering around $10 \mu \mathrm{m}$ from the sample. The excitation voltage is $1 \mathrm{mV}$. The values obtained are $\boldsymbol{C}=2.83 \times 10^{-15}$ and $\boldsymbol{\alpha}=4.69 \times 10^{-6} \mathrm{C} / \mathrm{m}$. 
We perform the same fitting to a QTF loaded with an actual tip immediately before the experiment. This fitting is shown in Figure 26. From the values $C$ and $\alpha$ values obtained above the resulting value of $\alpha=4.112 \times 10^{-6} \mathrm{C} / \mathrm{m}$ and of $1.66 \mathrm{~A} / \mathrm{m}$ for the ratio $\Delta I_{R M S} / \Delta x_{R M S}$. Another values found for a different QTF (of the same batch) were $\alpha=4.2510^{-6} \mathrm{C} / \mathrm{m}$ and $1.70 \mathrm{~A} / \mathrm{m}$. We have found that for QTF from the same batch the ratio $\Delta I_{R M S} / \Delta x_{R M S}$ has consistently in the $1.6-1.9 \mathrm{~A} / \mathrm{m}$ range when measured with the Nanonis OC-4 system (see chapter 5).

However, measurements with the SR-850 system have consistently yielded values between $2.9 \mathrm{~A} / \mathrm{m}$ and $3.2 \mathrm{~A} / \mathrm{m}$. For calculation purposes in this thesis we will use the average value of $1.75 \mathrm{~A} / \mathrm{m}$ value given by the measurements with OC- 4 . When fabricating the probes and mounting them on QTFs, we pursued maximizing the quality factor, $Q$, to obtain greater sensitivity. $Q$ represents the ratio of the energy stored and the energy dissipated in one cycle of oscillation. Since the higher the excitation voltage the larger the oscillation's amplitude, we are always looking to minimize the energy given to the QTF without sacrificing the signal to noise ratio (SNR).

For the actual ARCs measurements, unless otherwise indicated, we will use the WGAS sensor as the control signal. The response of the spectrum of the WGAS sensor is shown in Figure 27. The noise level (peak to peak) of the WGAS amplitude is $1 \%$ for every ARC performed in this thesis. 

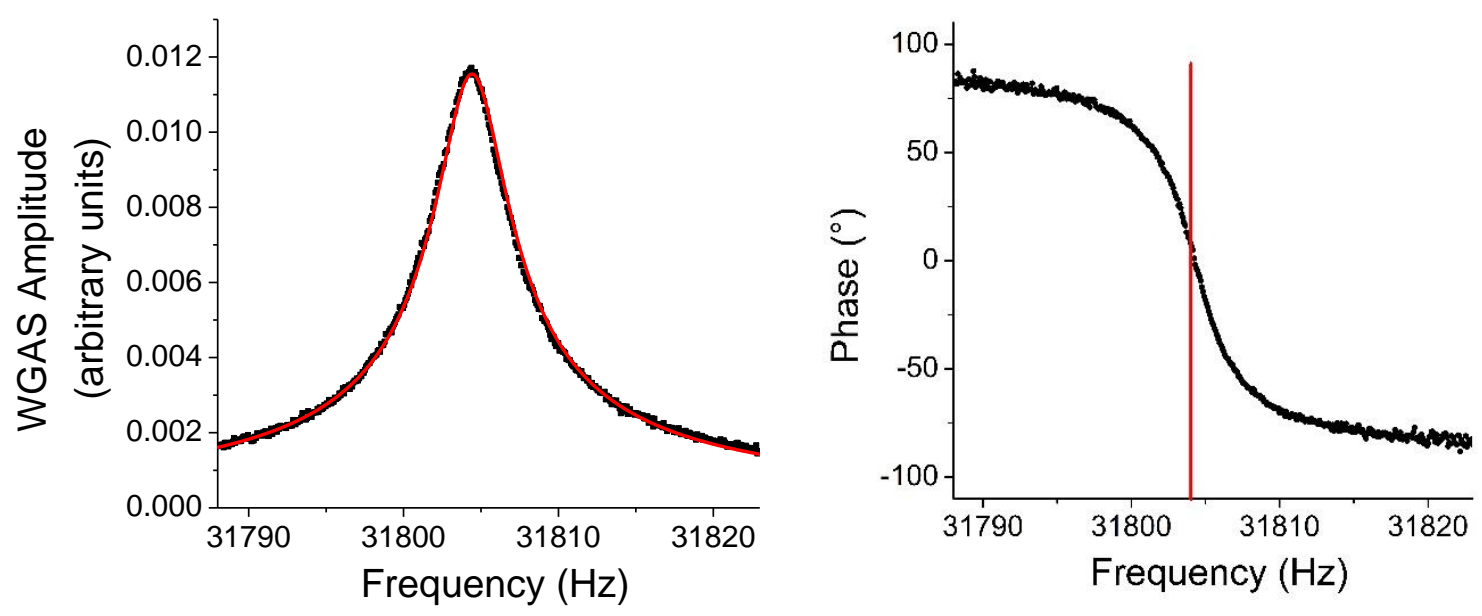

Figure 27. The amplitude and phase response of the WGAS signal. The red vertical line marks the resonance frequency.

\subsection{Tunneling current measurements}

Tunneling current measurements precedes the advent of atomic force microscopes and it is a technique that boasts great accuracy[62] due to the exponential nature of the tunneling current between two surfaces at close distance. This relationship holds especially in vacuum conditions where clean surfaces are easier to maintain. STM at ambient conditions presents the added difficulty of dealing with the adsorbed water layer[102]-[104]. Electrical conduction occurs through this layer mediated by ions.

Freund et al.[105] has shown two type of curves for high a low humidity environments in tunneling current experiments in SPM. 

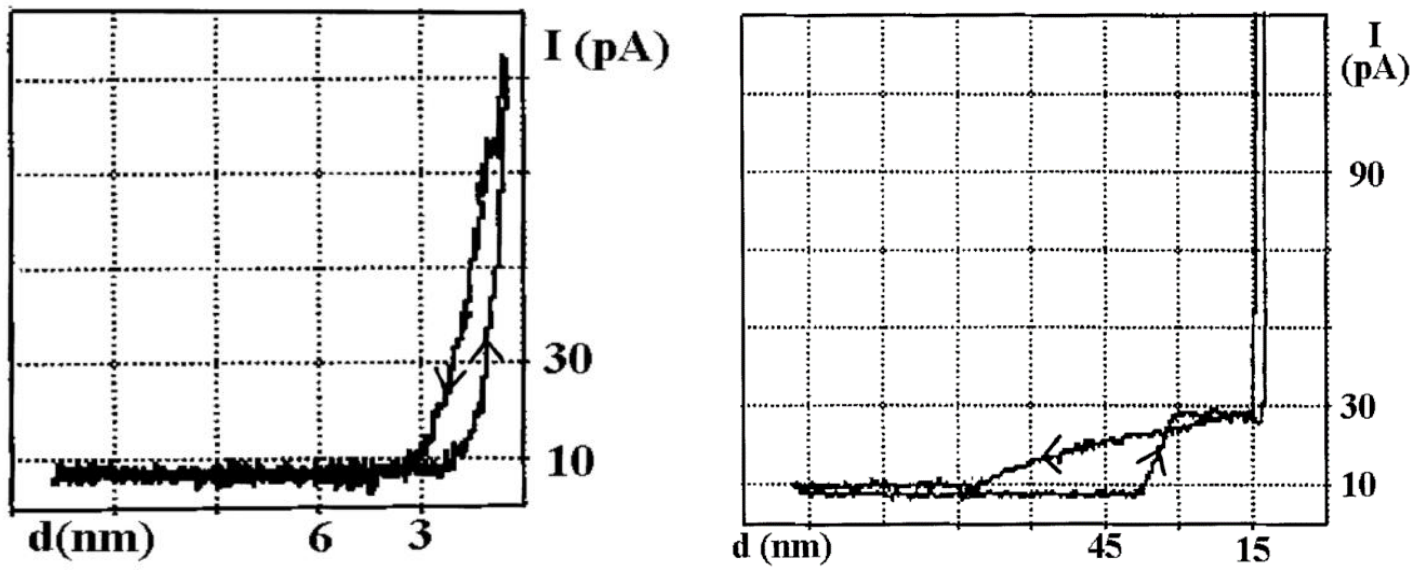

Figure 28. Graph from Freund et al. [105] showing the dependence of tunneling current and distance from the sample with low relative humidity (RH). There is no indication of sharp onset of tunneling current due to the water layer. Right, same process in high $\mathrm{RH}$ levels. There is the indication of the sudden jump in tunneling current when the probe enters the adsorbed water layer.

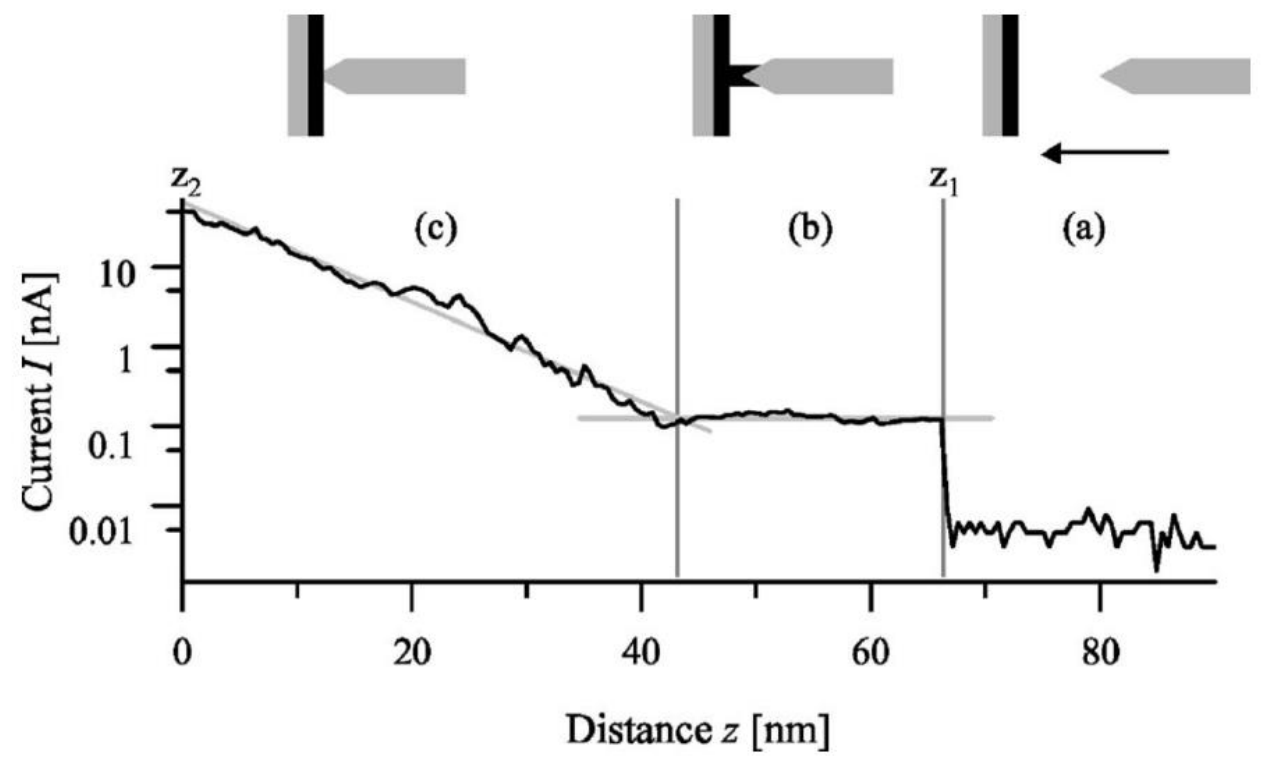

Figure 29. Graph from Opitz et al. [106] showing the behavior of a typical tunneling current approach curve at ambient conditions. 
Three regions seem to appear when an approaching and retracting curve is performed with an STM at ambient conditions, the initial jump to electrical conduction, the plateau and the finally the exponential increase in the tunneling current.

The probability of conductance is given by,

$$
\frac{I}{V}=e^{-2 z \sqrt{\frac{2 m \varphi}{\hbar^{2}}}}
$$

At ambient conditions the energy barrier cannot be directly related to the work function of the surface, and equation ( 16 ) describes an 'apparent barrier'.

From ( 16 ) we obtain,

$$
\begin{gathered}
\operatorname{Ln}(I)-\operatorname{Ln}(V)=-2 z \sqrt{\frac{2 m \varphi}{\hbar^{2}}} \\
\frac{\partial \operatorname{Ln}(I)}{\partial z}-\frac{\partial \operatorname{Ln}(V)}{\partial z}=-\sqrt{\frac{2 m \varphi}{\hbar^{2}}}
\end{gathered}
$$

And finally

$$
\frac{\hbar^{2}}{8 m}\left[\frac{\partial \operatorname{Ln}(I)}{\partial z}-\frac{\partial \operatorname{Ln}(V)}{\partial z}\right]^{2}=\varphi
$$


Entering the required values we have,

$$
\varphi=\left[\frac{1}{1.025} \frac{\partial \operatorname{Ln}(I / V)}{\partial z}\right]^{2}
$$

with $\varphi$ in eV and $z$ expressed in angstroms[107].

The experimental barrier height $\varphi$ calculated from the graph depends on the properties of the water layer. It is also striking how our results show a continuous increase on the tunneling current even after the tip has made contact with the surface (as evidenced by SEM images taken after the process). However, in this thesis we are mainly concerned with the exponential trend of the tunneling current as a technique to determine the surface's position (see APPENDIX C).

\subsection{Probe fabrication and inspection}

A $100 \mu \mathrm{m}$ diameter Pt-Ir $10 \%$ wire with $99.99 \%$ purity was etched with a solution of $12 \mathrm{~g}$ of calcium chloride dihydrate $\left(\mathrm{CaCl}_{2} \cdot 2 \mathrm{H}_{2} \mathrm{O}\right), 35 \mathrm{ml}$ of de-ionized water (DI) and $15 \mathrm{ml}$ of acetone. The acetone was added to minimize bubbling while etching. $\mathrm{A}$ carbon rod inside a beaker acted as the cathode. The frequency of the AC etching voltage was $60 \mathrm{~Hz}$ with. A preamplifier supplied the power from a signal generator. Applied voltages ranged from $1 \mathrm{~V}$ to $1.5 \mathrm{~V}$ in amplitude and from a few milliamps to tens of milliamps. 
A Field Emission Scanning Electron Microscope (FE-SEM) Hitachi S-4160 underwent major repairs after a systematic troubleshooting to provide the necessary probe characterization.

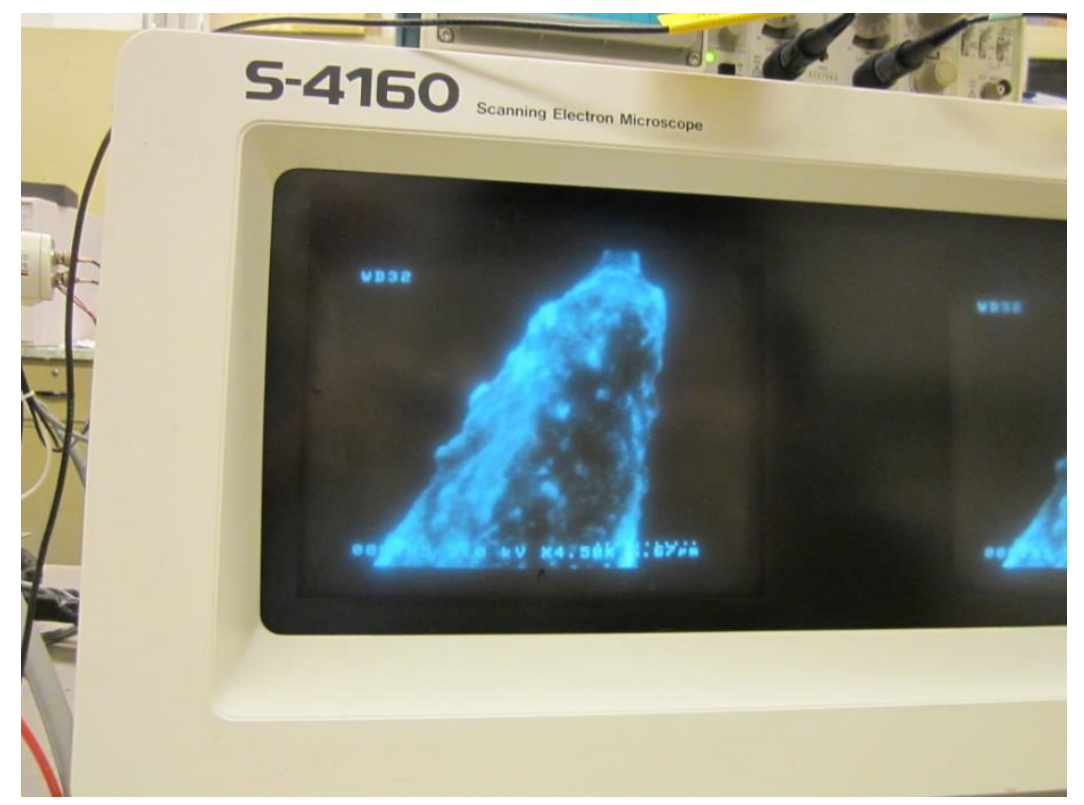

Figure 30. Monitor screen of the Field Emission Scanning Electron Microscope (FE-SEM) Hitachi S-4160 restored in our laboratory.

A LabVIEW acquisition program using a National Instruments acquisition card was used to digitize the SEM images. The calibration of the SEM images was done by imaging an AFM calibration grid with squares of $5 \mu \mathrm{m}$ side. 


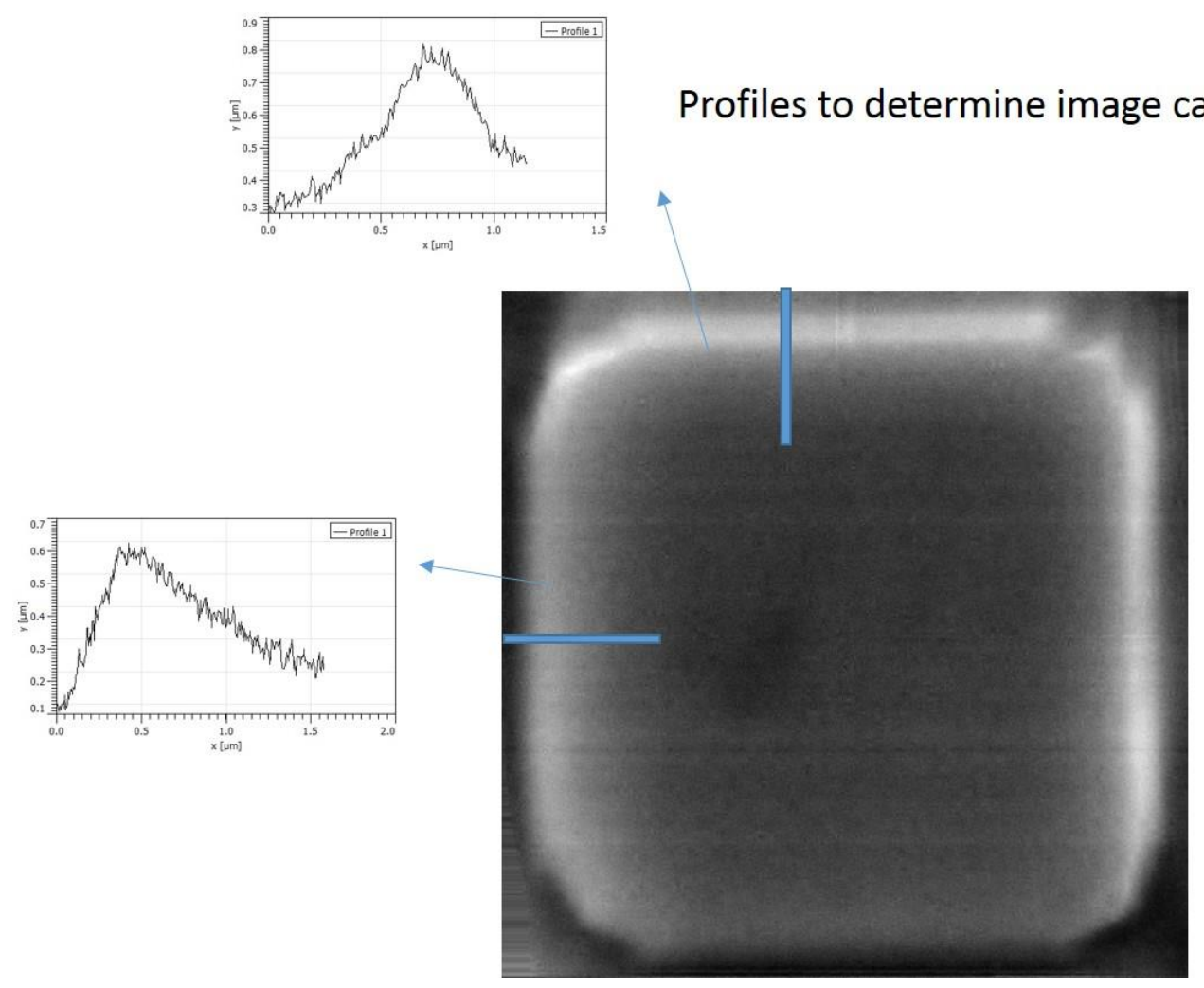

Figure 31. SEM image of the AFM calibration grid used for calibration of the images. The edges of the square were determined by taking the maximum peak in the case of a bright edge, and halfway the slope for a dim edge.

Immediately after etching the Pt-Ir probes were submerged in DI water while simultaneously placed in a sonicator. Right afterwards they were inserted in the FE-SEM chamber and imaged. As an example, Figure 32 shows an etched Pt-Ir probe mounted on a QTF ready to be used for and ARC. 

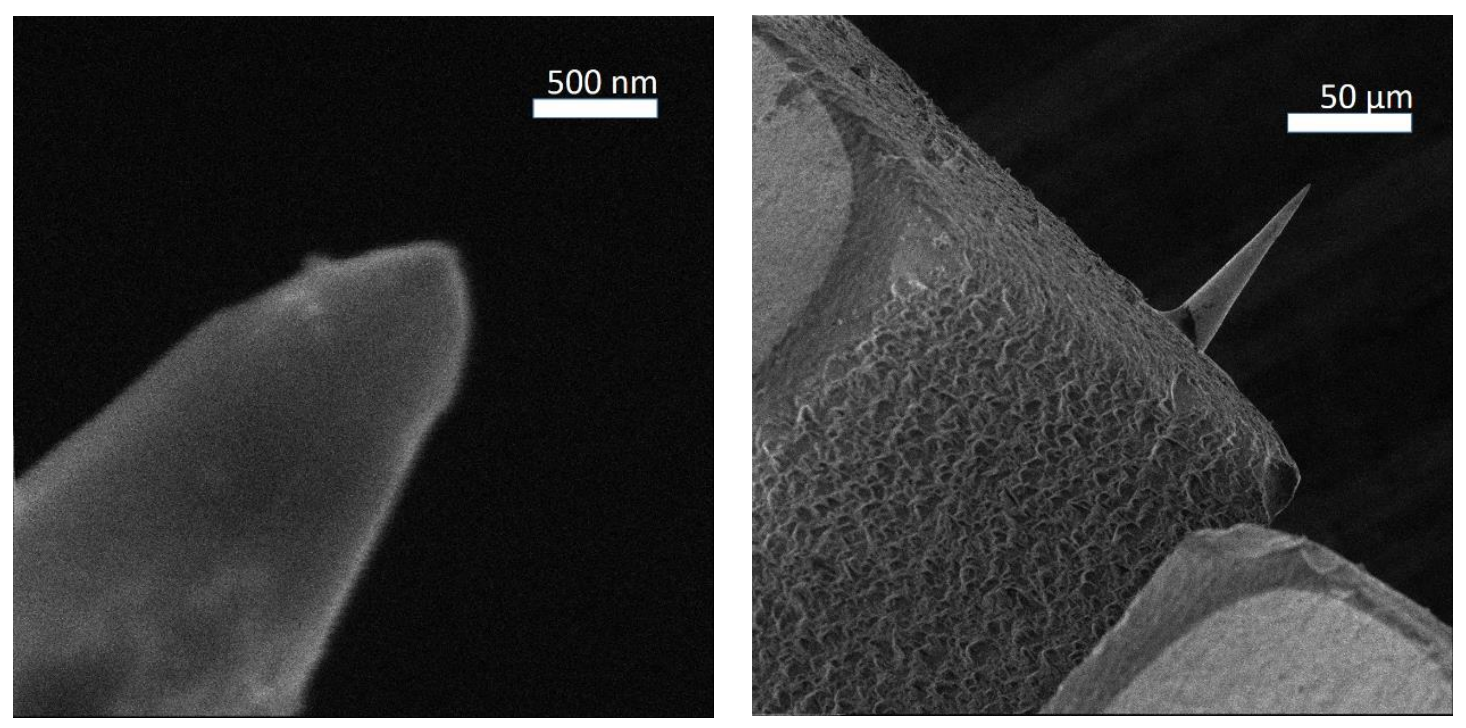

Figure 32. FE-SEM images of a test probe before the experiment. Right: The probe protrudes approximately $70 \mu \mathrm{m}$ from the edge of the tine of the QTF. 


\section{CHAPTER 4}

\section{CALIBRATION and DRIFT CORRECTION}

The WGAS-SANM QTF-SPM system was initially designed to perform measurements in biological samples and to work in tandem with an optical inverted microscope for near-field fluorescence microscopy applications, where many application would require resolution in the $20 \mathrm{~nm}$ scale. The $20 \mathrm{~nm}$ mark was set by the minimum near-field aperture size that can be fabricated (within a routine process). Hence, the microscope stages were a bit relaxed from Angstrom precision measurements. However, as the properties of the adsorbed water layer (involved in the near-field imaging) appeared to influence the metrology capabilities of the system, the research work evolved towards characterizing such a fluid-like layer. The thickness of this layer is in the order of $6 \mathrm{~nm}$ scale (but could be smaller or greater depending on the humidity) and the need of precise localization of the layer (ideally with sub-nanometer precision). To work with this $\mathrm{nm}$ precision, this thesis focused its efforts to upgrade the sensitivity of all sensing elements and developed protocols (inside its electronics controls) to overcome the potential thermal drifts.

The MCL Nano-Z50HS piezo stage while having great time response and a large range, lacks the necessary resolution for the noise level of the available driving voltage. A simple calculation (dividing the full range of the input driving signal over the full

displacement range $\left.\frac{20}{5 \times 10^{5}} \frac{V}{\AA}\right)$ puts the equivalent noise of the driving signal at $40 \mu \mathrm{V}$ 
peak to peak to obtain one angstrom precision. Keeping in mind that the maximum resolution of the system is placed at exactly one angstrom by the manufacturer and that to reach such levels a voltage divider arrangement would place the range of the piezo stage at $660 \mathrm{~nm}$ (assuming a noise level of $3 \mathrm{mV}$ for a regular driving signal).

This discussion leads us to the issue of thermal drift. The response of the stage to thermal drift was not optimized beyond nanometer considerations. The rate of thermal drift between the probe and sample has been measured to be in a range between 2 and 15 angstrom per second. Considering that the measurements could take several hours, the amount of thermal drift can easily reach tens or even hundreds of nanometers. This would put the $660 \mathrm{~nm}$ range discussed above within dangerous limits. An alternative for position control was the use of piezotubes which have long been used in STM measurements. Their sensitivity depends on their geometry and the mechanical load placed on them. Their short range while a disadvantage, means that to obtain angstrom resolution a noise level of a few millivolts is tolerable. In our case, our calibration showed that a $1 \mathrm{mV}$ peak to peak driving voltage is equivalent to a motion of $0.32 \AA$.

In this work we set to implement a low noise amplifier of gain 2 to solve the driving voltage noise problem and a software correction technique to overcome the thermal drift of the system. This drift compensation technique requires calibrating the displacement of the PZT for hysteresis and creep. With this in mind, the feedback mechanism is used along with the closed loop integrated Nano-Z5OHS stage, with a calibrated total range of $50,752 \mu \mathrm{m}$ for the $-10 \mathrm{~V}$ to $+10 \mathrm{~V}$ range. The associated strain 
gauge has a total range of $0 \mathrm{~V}$ to $+10 \mathrm{~V}$. To drive the input we used the SR 560 from Stanford Research with a low pass filter. This assures a precise driving of the NanoZ50HS stage. The strain gauge output is amplified with the instrumentation amplifier INA127 form Texas Instruments. The gain of the circuit is 10 and the offset voltage is controlled with the LabVIEW PZT calibration program from section 3.3.2.

The precision of the strain gauge measurement is given by $50,752 \mathrm{~nm} / 10 \mathrm{~V}=$ $5,075.2 \mathrm{~nm} / \mathrm{V}$. After the differential amplifier the value is $1.97 \times 10^{-3} \mathrm{~V} / \mathrm{nm}$. The 16 bit analog input from the FPGA PCle $-7852 \mathrm{R}$ measures the voltage and the minimum voltage value it can measure is $20 / 65,535 \mathrm{~V}=0.3052 \mathrm{mV}$. A low pass digital filter was implemented with a cut of frequency of $15 \mathrm{~Hz}$ and $4^{\text {th }}$ order Butterworth filter. A 0.3052 $\mathrm{mV}$ value from the amplified strain gauge is equivalent to $0.3052 \mathrm{mV} /(0.00197 \mathrm{~V} / \mathrm{nm})$ $=0.155 \mathrm{~nm}$.

\subsection{Calibration of PZT with feedback mode}

Most commercial piezo stages use mechanical displacement multipliers to achieve high precision displacement measurements. In this fashion capacitance sensors and/or strain gauge sensors are adequate to measure sub-nanometer displacements, providing the signal for the feedback control for their closed loop displacement systems. Hysteresis and creep are always present in a PZT due to domain type structure inherent in piezo materials. Charge actuators have proven to be more effective in correcting for hysteresis[108] but its implementation requires appropriate circuitry. Here we use the 
feedback mechanism, a MCL closed loop control stage (Nano-Z5OHS) and the FPGA implementation to combine feedback and QTF precision to measure the real position of the probe. The procedure includes three steps:

Step 1: Approaching the probe, which is mounted on the piezotube with a QTF as a transducer (Figure 33), towards a sample securely placed on the MCL Z scanner. The MCL scanner has a strain gauge that can determine the position of the stage with 0.16 $\mathrm{nm}$ precision (with our instruments, as seen in the previous section).

Step 2: The probe is maintained inside the adsorbed layer with a stable feedback (determined by the error signal).

Step 3: Having the PZT coupled to the MCL Z stage through the probe in feedback, the $M C L$ is made to scan with constant velocity between an initial and final positions. There is one trace moving forward and one moving backwards as shown in Figure 35.

Critical to the calibration technique is a proper feedback signal. Probes are prone to change its interaction with the adsorbed layer and thus a stable feedback assures that the distance is being approximately kept constant. Only the curves with a stable error signal were selected for the calibration's calculations. Since the SPM frame was designed for imaging of objects in with dimensions in the microns, it was not optimized for low drift when initially designed. As a consequence a significant drift (fraction of nanometers per second) is caused by temperature changes, affecting the tip-sample distance during the measurements. 


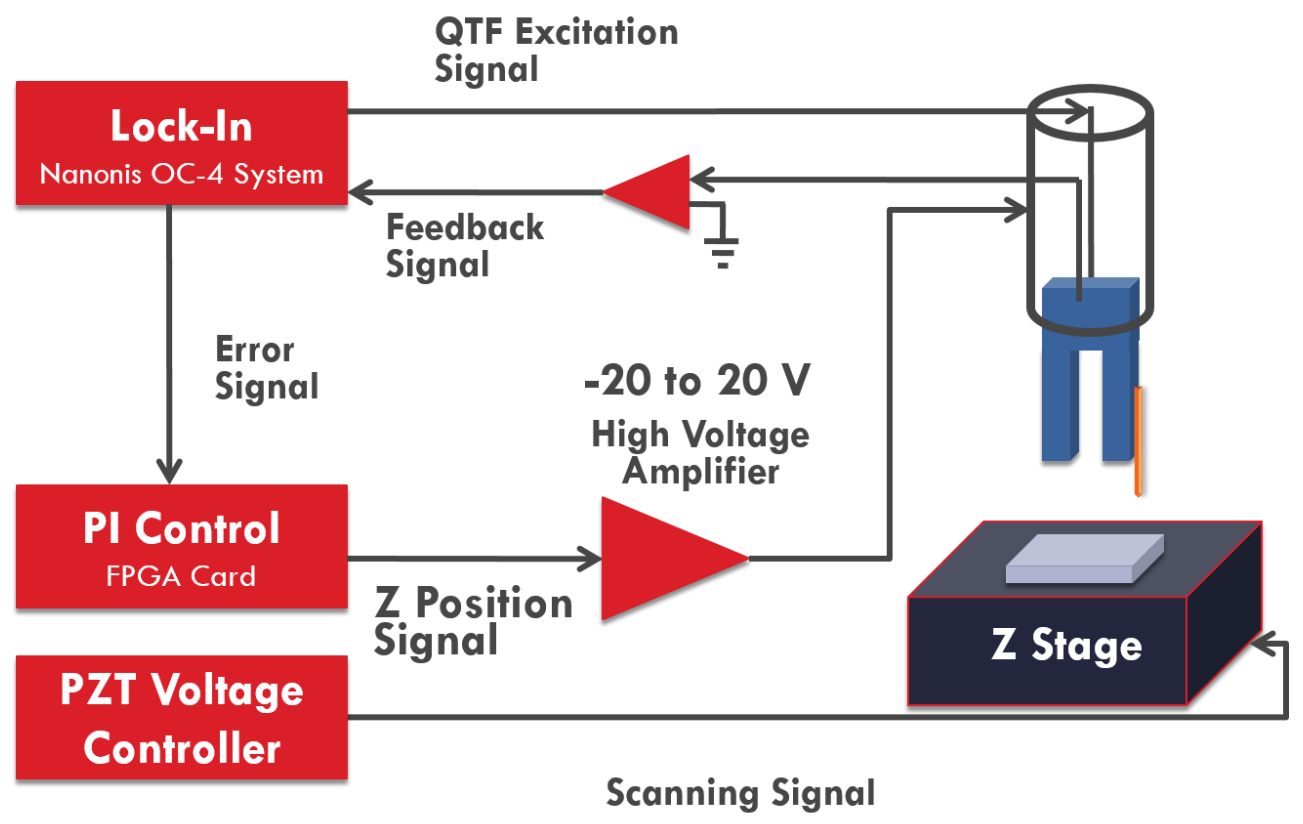

Figure 33. Experimental setup for the PZT calibration procedure.

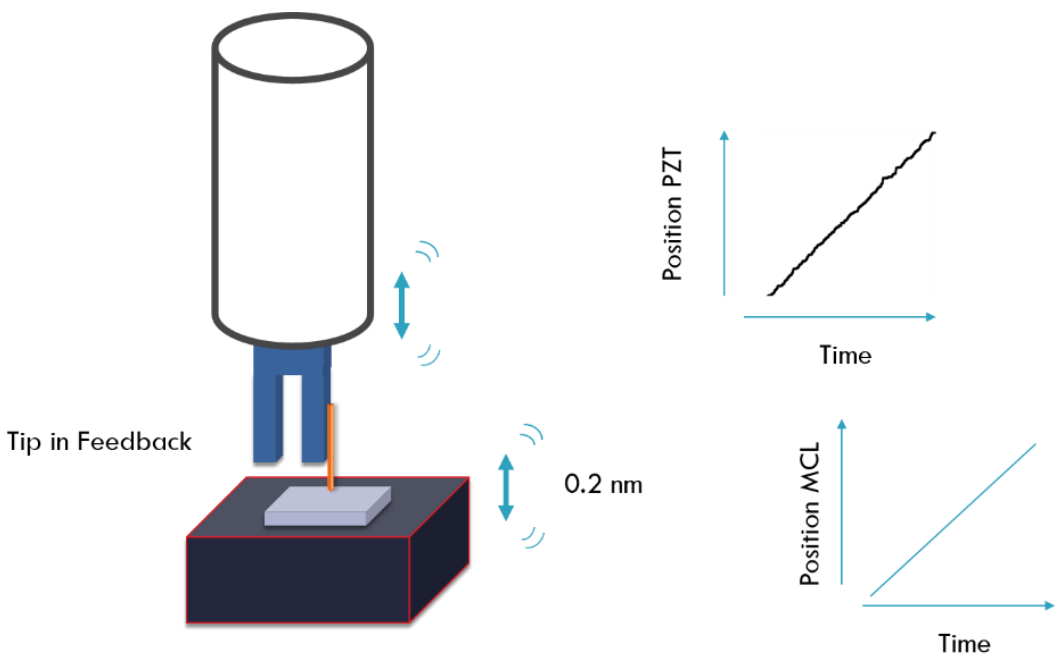

Figure 34. Schematic representation of the PZT calibration process. When the probe is in feedback, the PZT tracks the motion of the surface, which rests on a comercial piezo-stage with closed loop positioning. 


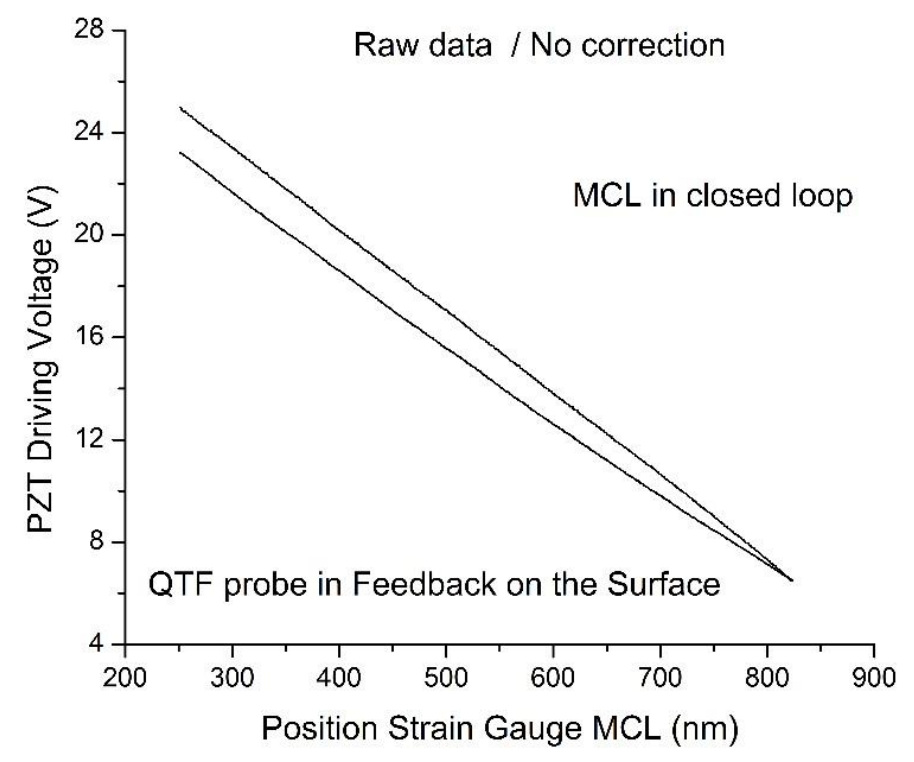

Figure 35. Raw data of the PZT driving voltage in the calibration measurement process. The beginning and ending values of the PZT driving voltage do not coincide. As expected the system has drifted. Since the MCL piezostage determines its position with closed loop control the cause of the discrepancy must be thermal drift of the stage where the PZT is mounted on.

Results of the scanning motion of the MCL. The $Y$ axis shows the driving voltage of the PZT. Since the drift is approximately constant we can assume a constant change in the position caused by it. If we subtract this position, $v(t) t$, and find the value of $v(t)$ that will make the two ends of the curve coincide (which should be the results if drift is not present) then we would have the average drift velocity. The corrected graph is shown below. 


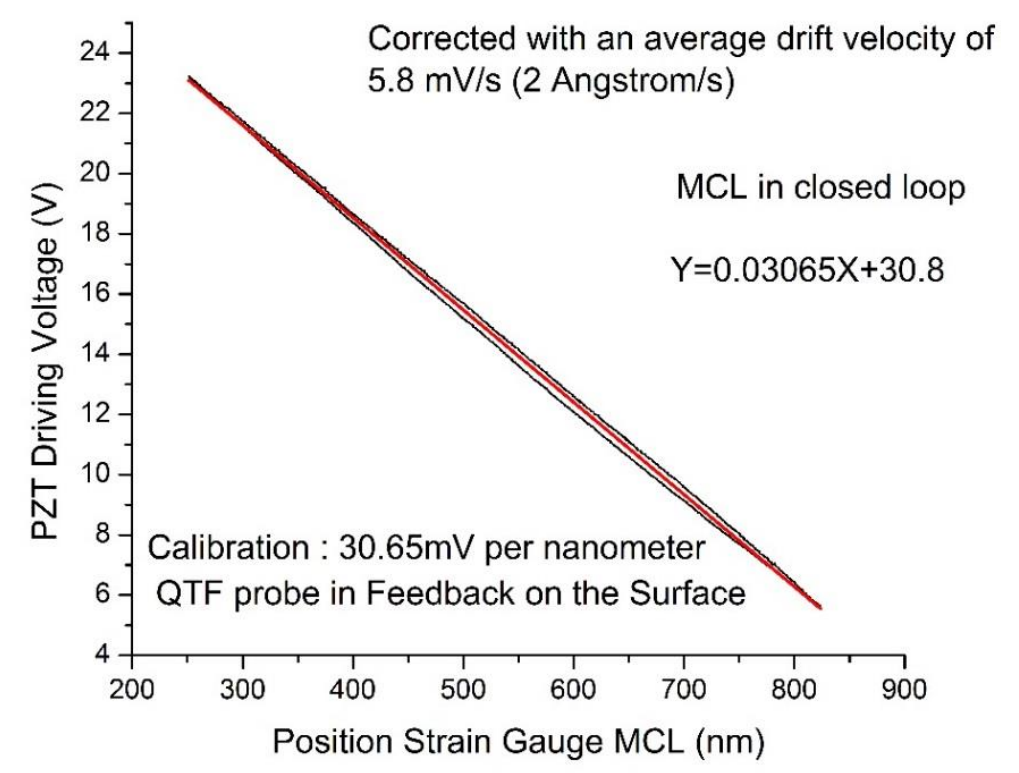

Figure 36. Hysteresis curve after the drift velocity correction.

From the calibration curve we can obtain the hysteresis curve to correct for the position post-measurement. The fitted line is directly subtracted from the hysteresis curve and a deformed ellipse is obtained. Instead of trying to fit the curve to a rotated ellipse, the curve is fitted to a $3^{\text {rd }}$ degree polynomial and the result is used for correcting the position of the PZT using the driving signal. The hysteresis of the approach and retraction parts are averaged. The somewhat irregular pattern of resultant curve may be caused by a combination of small feedback oscillations, slight change in drift velocity, hysteresis of the adsorbed layer and the lack of rotational operation before performing the subtraction. Figure 37 shows the hysteresis curve after the fitted linear curve has 
been subtracted. This curve is then fitted with a polynomial curve to be used as the correction factor (Figure 38).

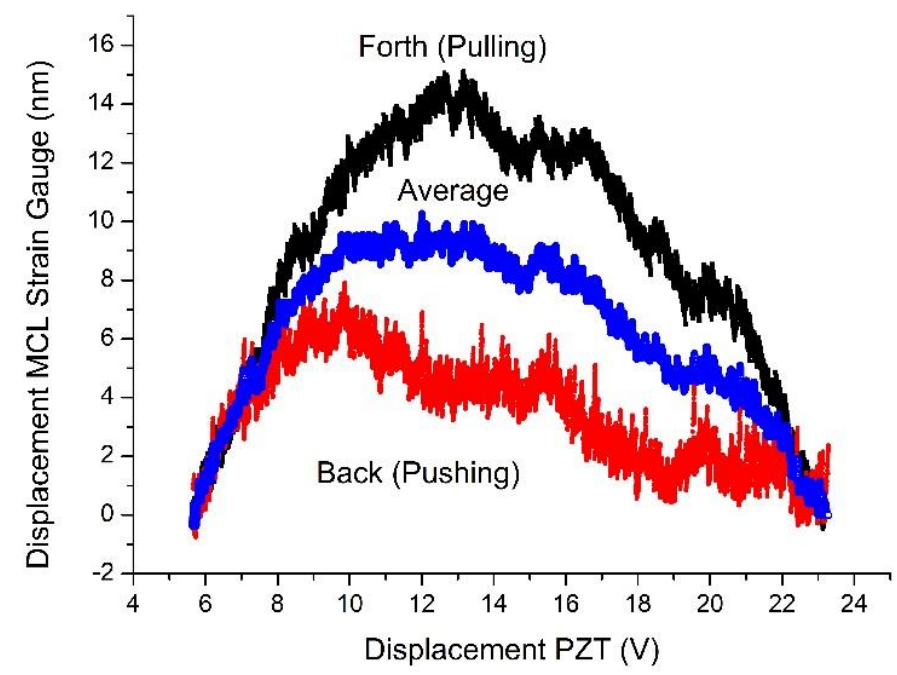

Figure 37. Resulting curves obtained from substracting the fitted linear curve from the experimental hysteresis curve. The average of both curves is also shown.

With this fitted curve the position of the QTF is determined both in the extension and the contraction. We calculated the displacement associated with the fitted linear curve Figure 36 . From the total voltage change, $17.65 \mathrm{~V}$, we obtain $17.56 / 0.03065=$ $575.9 \mathrm{~nm}$ of total displacement during the measurement. Since the shape of the hysteresis curve is approximately constant for low voltages $(<100 \mathrm{~V})$ the curve can be proportionally fitted to any particular displacement length just by enlarging or reducing the fitted hysteresis correction. 


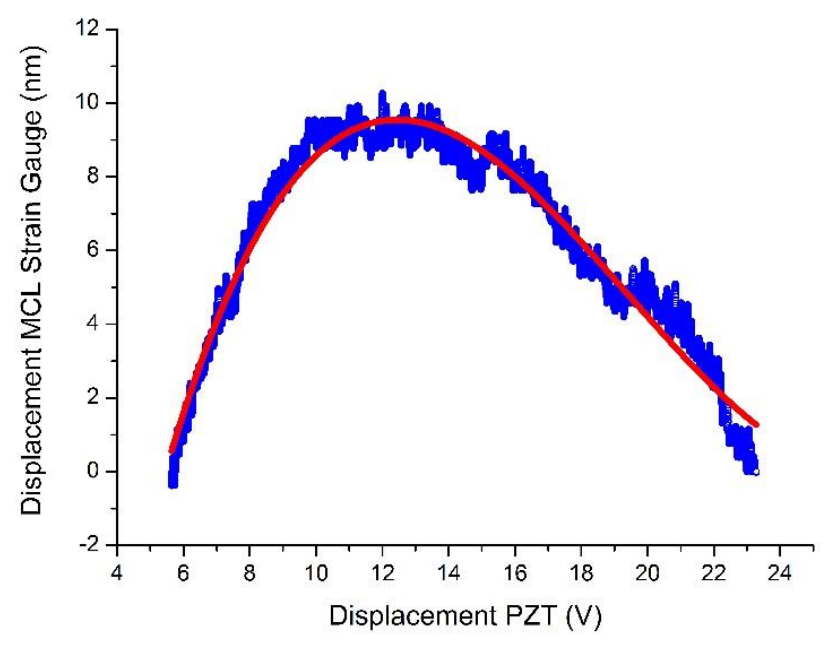

Figure 38. Fitted curve of the average PZT hysteresis.

The goal of this calculation is not to provide an accurate function to describe the hysteresis of the PZT. Instead it provides an approximate value of the error obtained when we use the driving voltage as proportional to the position. Figure 37 shows that the maximum displacement due to hysteresis is $14 \mathrm{~nm}$ per $575.9 \mathrm{~nm}$. This provides an over/under estimation of the position of $2.4 \%$. With this ratio we can estimate the error when performing an approaching and retracting curve (ARC) of $10 \mathrm{~nm}$ in length, resulting in $10 \mathrm{~nm} \times 0.024=0.24 \mathrm{~nm}$. In the case of a displacement of $100 \mathrm{~nm}$ the error in the estimation is $2.4 \mathrm{~nm}$. This calculations offers of course the case were feedback effects are not considered, in which case the maximum displacement would be smaller than $14 \mathrm{~nm}$. Using both the average of the back and forth curves and the calibration curve we obtain Figure 38. 
Another source of nonlinearity in piezo actuators is creep. It consists in the increase of the PZT expansion or contraction after has finished its change and is held at a constant value. The creep always increases in the direction of the change in applied voltage[109]. Depending on the material creep can significantly affect a measurement. We needed to know if is a factor while performing an approaching and retracting curve (ARC).

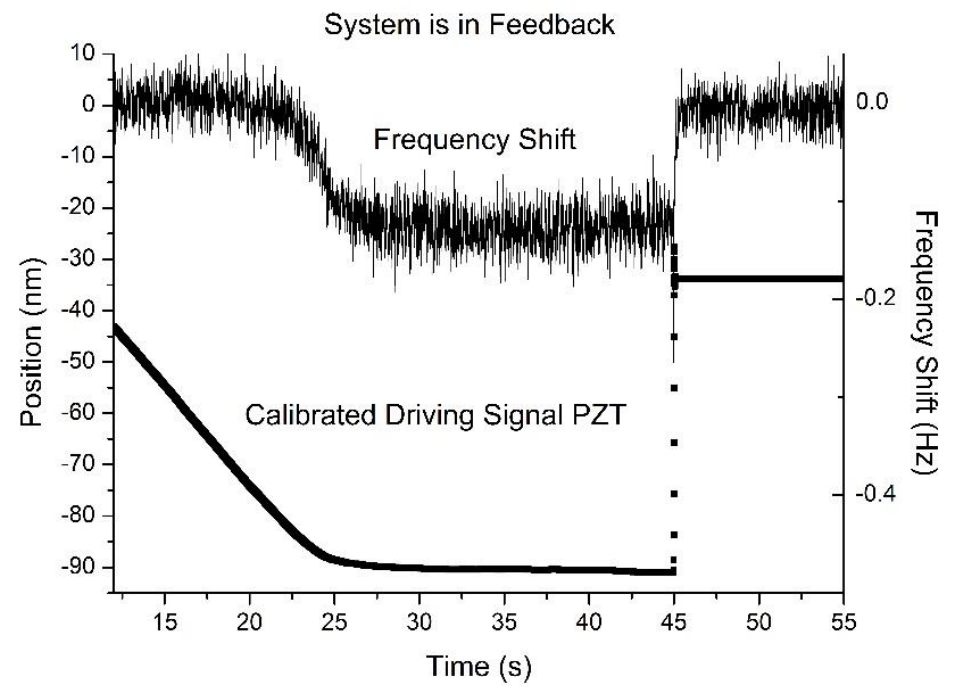

Figure 39. Top: Frequency shift as control signal for feedback control. The calibration driving signal of the piezotube is shown below. The probe is in feedback after second 25 and it follows the drift of the surface.

Figure 39 shows the drift estimation process. Around the fifteenth second the probe is approaching towards the adsorbed layer and at approximately twenty-fifth second settles into the position were the control signal reaches the predetermined setpoint 
inside the adsorbed layer (around $-160 \mathrm{mHz}$ ) achieving feedback. While the probe is in feedback the driving voltage remains approximately constant, and the control signal (frequency shift) drops to a value of $-160 \mathrm{mHz}$ (setpoint). The average value of the PZT driving voltage is recorded.

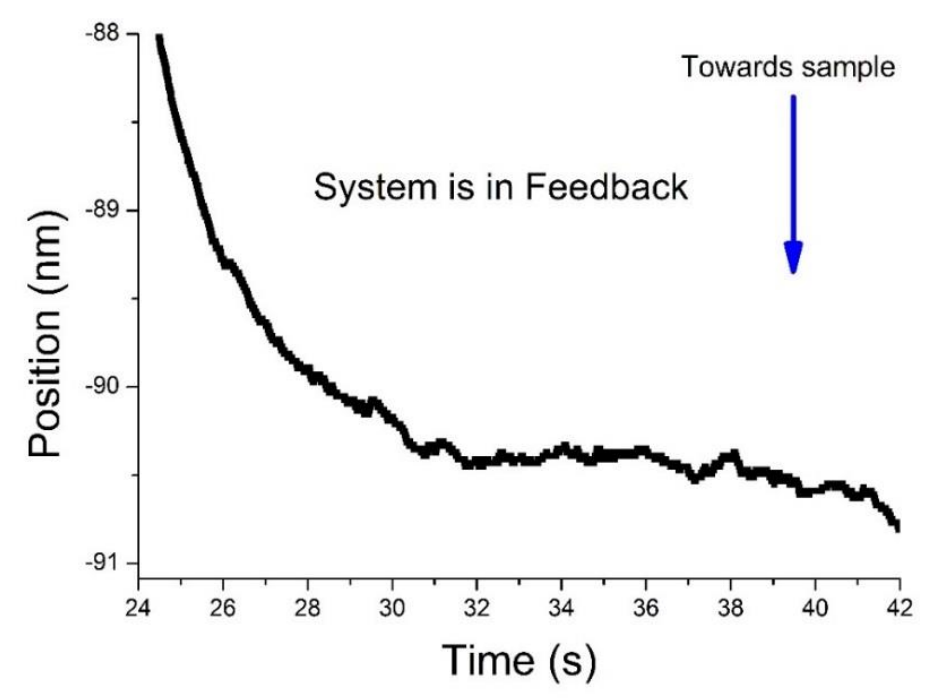

Figure 40. Same positioning curve showing the moment when the probe enters in contact with the surface. The effect of creep, while in feedback, is expected to bring the probe closer to the sample showing a decrease in the control signal (in an attempt to pull away from the surface) but this behavior is not observed. The slow downward change is attributed to thermal drift.

For this purpose a portion of the drift correction in one of the experimental ARC process was analyzed. There are a few observations from Figure 41. First, after contact, the PZT starts following the surface motion. However both the creep and the drift should be included in the final motion of the probe. This is the reason why instead of just 
calculating the slope from one measurement the drift correction process involves two independent measurements of the position in feedback and taking the average position in both instances.

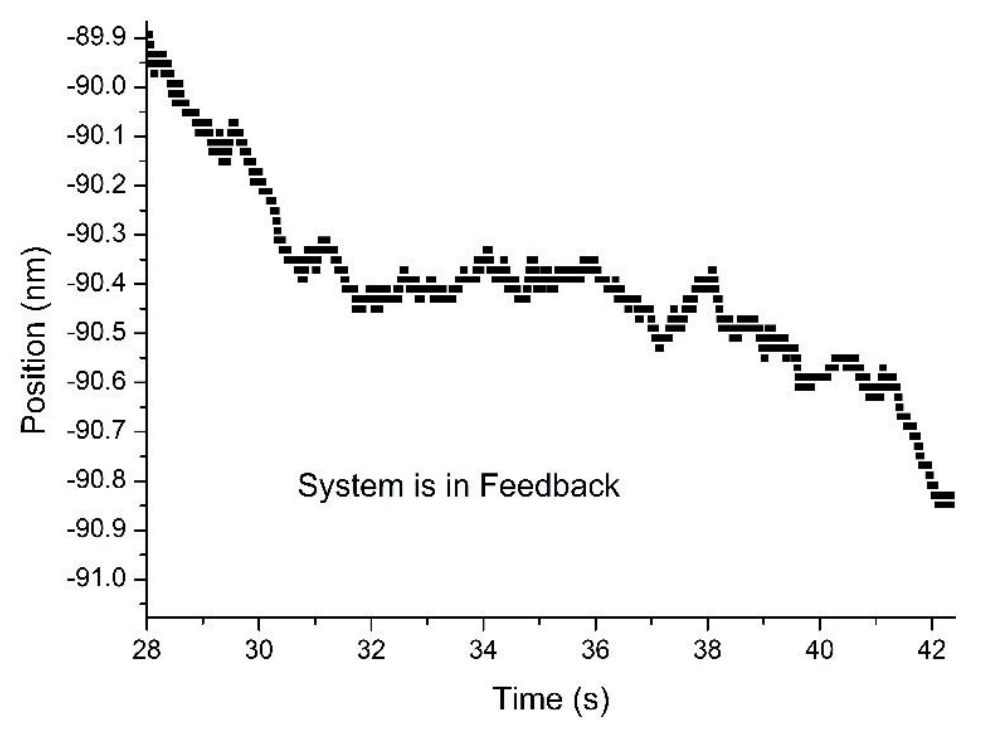

Figure 41. Same PZT positioning curve from Figure 39 and Figure 40. The voltage variation (peak to peak uncertainty in the position) is roughly $1 \AA$.

Another important feature is the uncertainty in the voltage signal (already multiplied by the calibration performed with the hysteresis fitting). It represents a variation of approximately 1 angstrom. This results validates the claim of atomic resolution in the position. The feedback process requires a signal to have the appropriate $\mathrm{S} / \mathrm{N}$ ratio and bandwidth. For the driving PZT signal to be able to hold the 
position of the PZT under conditions of surface interactions, both the control signal and the actuator response need to have a low and comparable SNR.

Another issue is the time response. Below are the MCL's strain gauge signal (which represents the real position) and the PZT driving voltage (while in feedback). Both signals have the same slope (when corrected for hysteresis) and overall behavior but the PZT curve is displaced about 122 ms to the right. This delay could come from either the feedback signal or the PZT response.

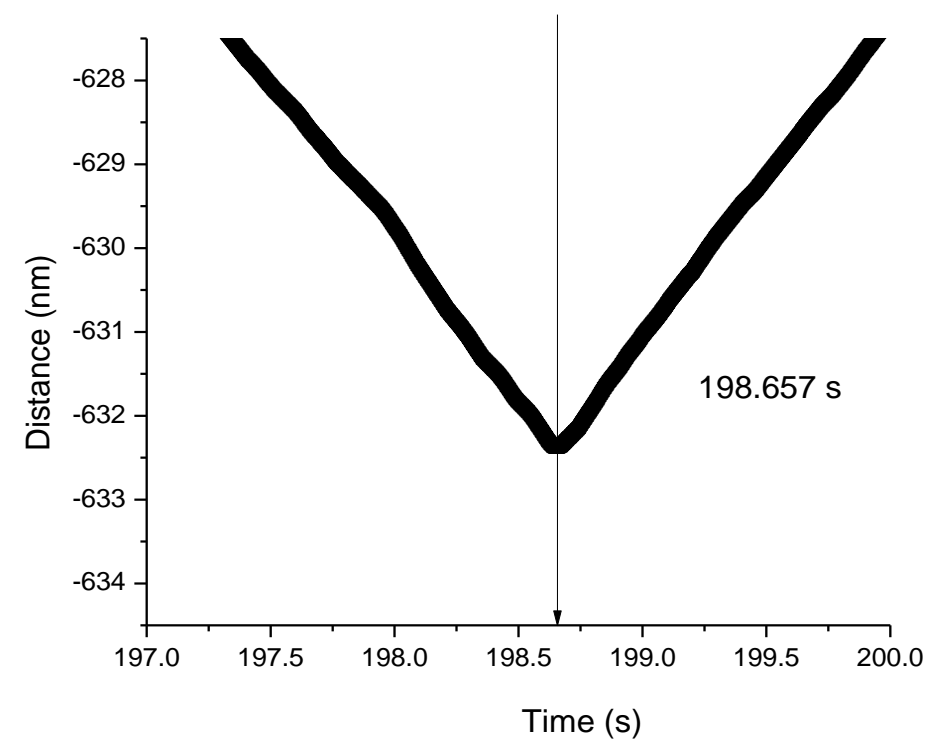

Figure 42. Signal from the strain gauge, showing the change in the position of the MCL piezo-stage while in feedback. 
However, if we look at the simultaneously measured control signal in Figure 44, it takes about $110 \mathrm{~ms}$ for the control signal to reach the setpoint. This means that the approximate PZT time response is about $12 \mathrm{~ms}$.

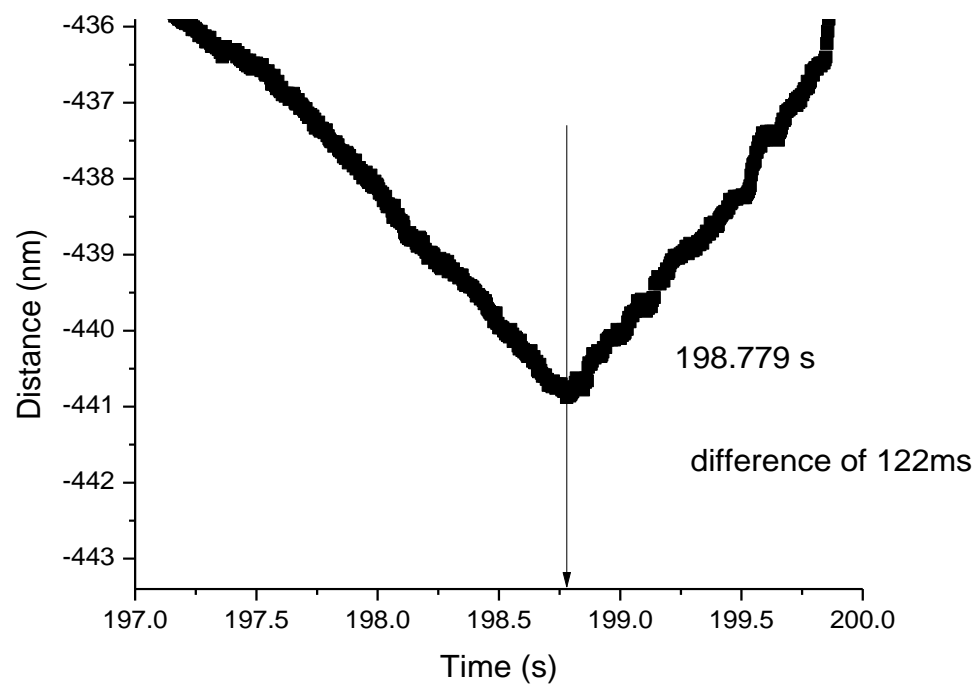

Figure 43. Driving signal of the PZT, while in feedback. The curves show that there is a delay of $122 \mathrm{~ms}$ for a velocity of $4 \mathrm{~nm} / \mathrm{s}$. The actual experiments were performed with a $2 \mathrm{~nm} / \mathrm{s}$ velocity approach which would amount to about $61 \mathrm{~ms}$ between the actual changes in the position.

If we consider that at a velocity of $2 \mathrm{~nm} / \mathrm{s}$, which is the average velocity used in our experiments, $12 \mathrm{~ms}$ corresponds to a traveled distance of approximately 0.25 angstroms then it can be safely assumed that the PZT does not play a significant role in any distortion that appears in the measurements. More significant are the time response of the frequency shift signal. 


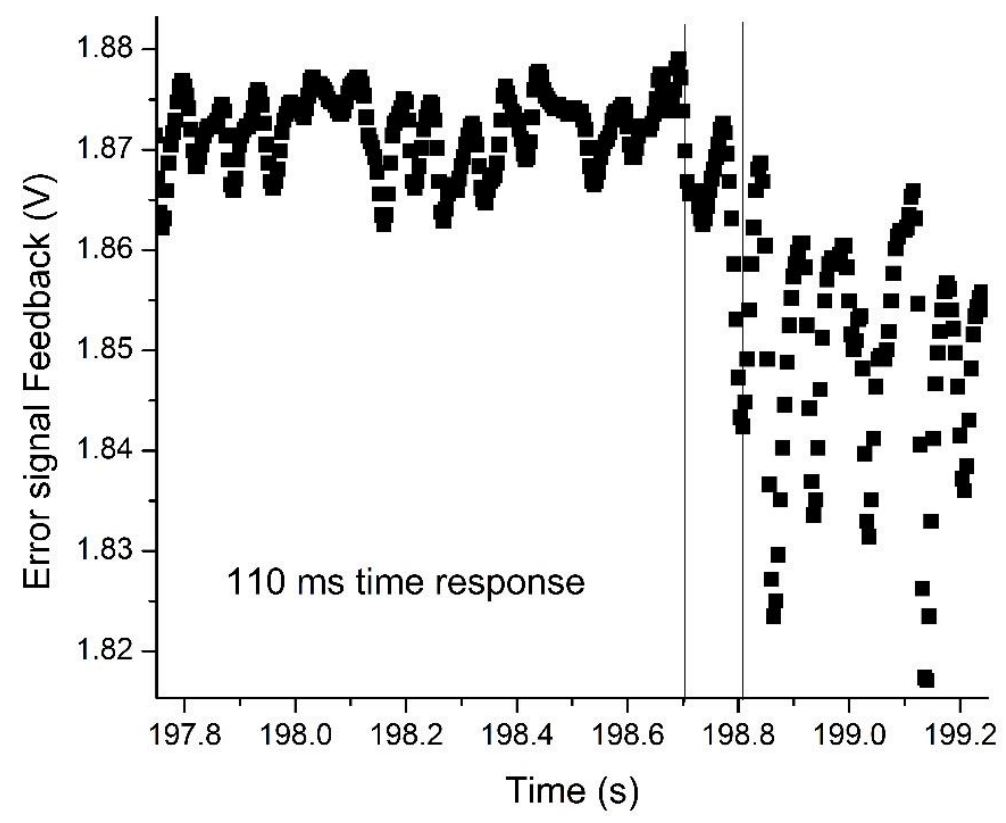

Figure 44. Signal of the feedback error signal for the calibration process. The graph shows the time response of the frequency shift signal. It is around 100 ms, which was intentionally tuned to have the greatest precision at the cost of time response. This means that only approximately $12 \mathrm{~ms}$ correspond to the PZT response time.

\subsection{Approaching and retracting curves operation}

In a regular mode the approaching and retracting control program (ARCP) initiates by approaching the surface at a predetermined velocity. After the control signal changes $0.5 \%$ or less in full range value, the system retracts the probe and performs the approach and retraction curve. In drift control mode the program uses feedback with a series of controlled approaches and retractions to calculate the drift. 


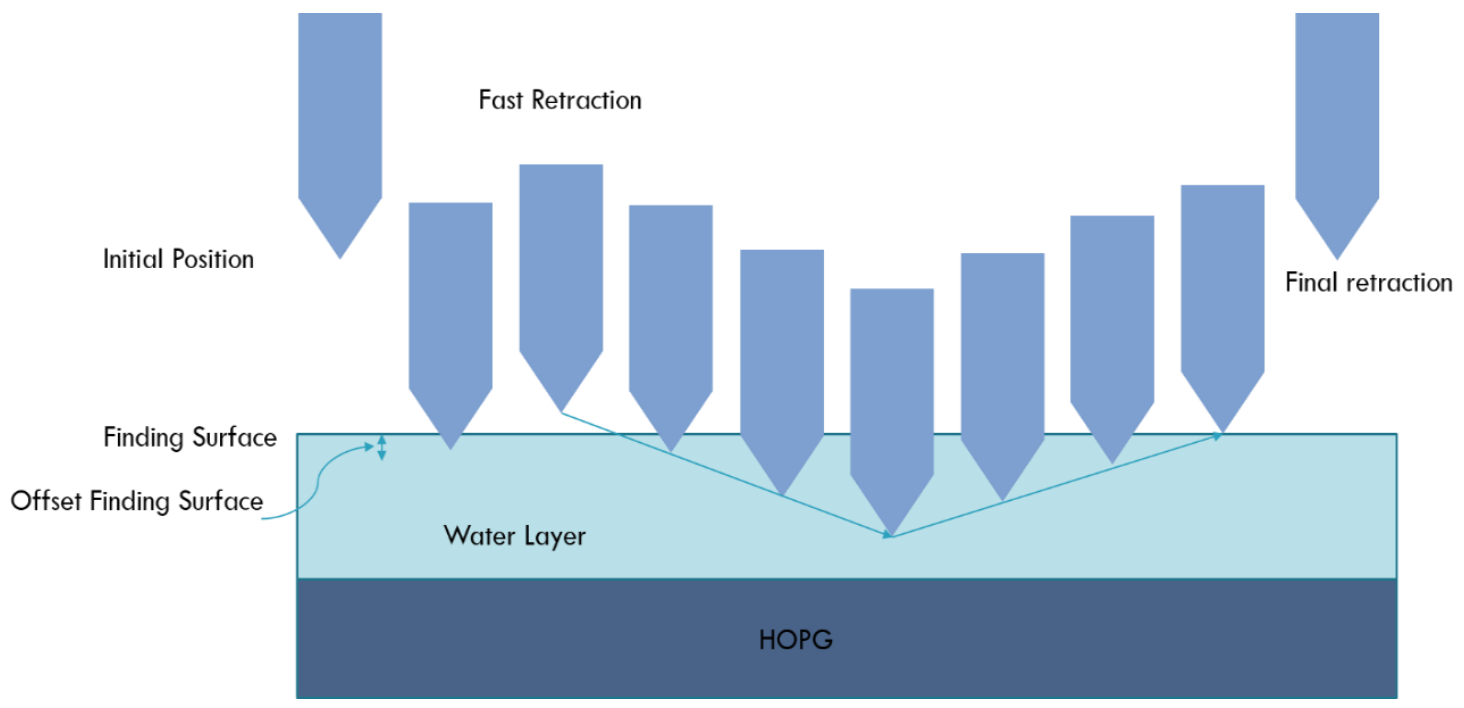

Figure 45. The process of approaching and retracting curves (ARC). After the air-fluid interface is found, (approaching the surface with $5 \mathrm{~nm} / \mathrm{s}$ speed), a fast retraction follows and then the actual ARC is performed. When the acquisition is finished the tip is retracted and the cycle starts again.

The ARC program includes a drift correction routine which corrects the values of the velocities to account for a constant drift velocity. It also includes Direct Memory Allocation (DMA) for the data transfer, optimizing the data acquisition without interfering with the control process.

Let us examine the process of an approach and retract curve. First, the probe is mechanically placed above the desired region. With the help of the camera a rough approach is made. The reflection of the QTF on the sample, caused by the relative smoothness of the surface, helps finding the appropriate position. Normally the probe is placed around 3 to 4 times the probes diameter. When this conditions are met, press 
the approach button. The approach process, will try and find the surface by approaching certain amount of steps with the stepper motor and then approaching with the PZT. If it does not find the surface the stepper motor approaches again. The distance traveled in each step with the help of the stepper motor has to be equal or less than the full range of the PZT, of course.

Reaching the surface, means that the signal from the QTF (whether it is frequency shift, amplitude or phase) changes to a predetermined setpoint (this is the main parameter typed by the user). Immediately after the surface is reached the surface retracts by a certain distance (this is the second parameter introduced by the user). The approach starts again, this time with a lower velocity. This portion is called 'Fine Approach'. Another setpoint will be reached (this the third parameter) and the software will retract the probe, and this is termed the 'Fine Retraction'. When the signal returns to the value it had before any interaction with the surface (signal at 'infinity') there is another sudden retraction to assure total breakage between the probe and sample interactions.

\subsection{Drift Compensation Mode}

The drift compensation mechanism uses the feedback control of the SPM to keep a constant distance from the sample. Thus any change in the position of the PZT within a small time period can be determined (drift). The process is done before every 
curve and improves the precision of the measurement, filtering out thermal drift from the stage where the sample rests.

\section{Drift Estimation}

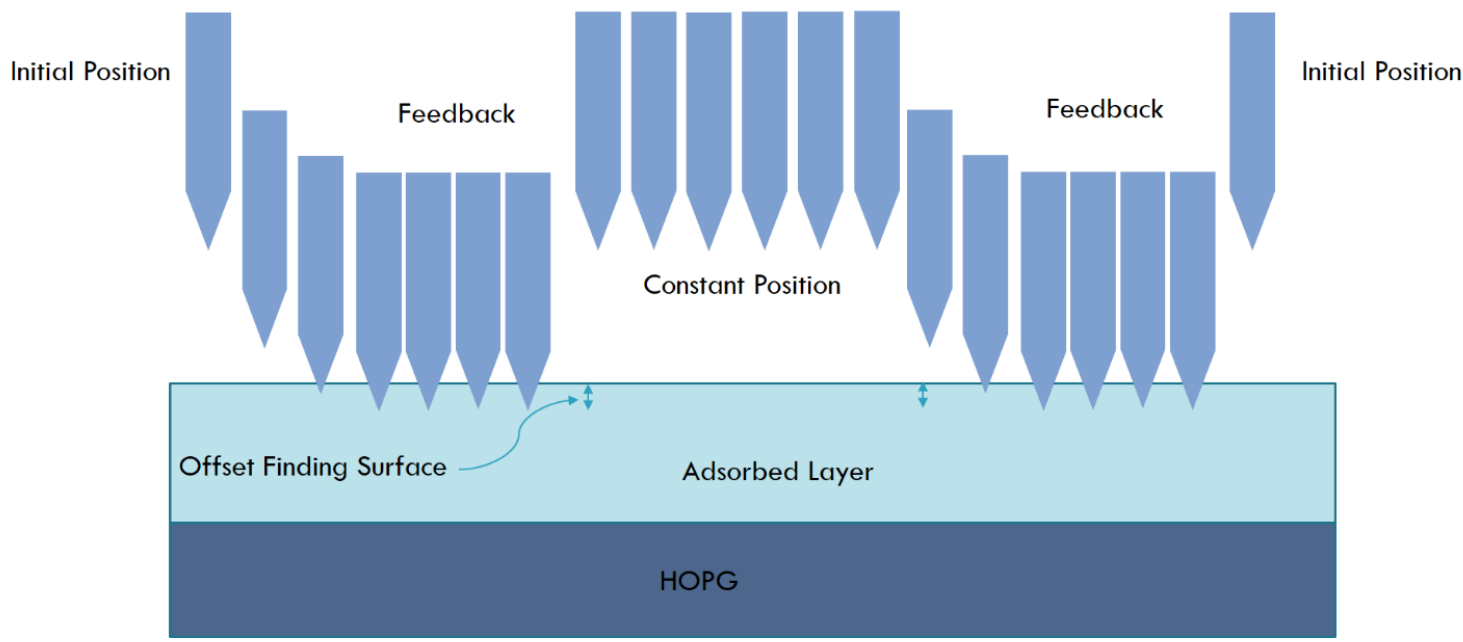

Figure 46. Procedure to estimate the value of the drift. After finding the surface and retracting (initial position) the feedback is turned on and the tip will approach the sample, stay in the predetermined feedback distance and the average value is recorded. Then the tip is moved to a known constant position away from the surface. Finally the tip is moved back into feedback and the position recorded again. If there were no drift, both initial and final positions would be equal, but because of the drift the difference is different than zero. Since the time between the two events is known, the calculation of the drift velocity is straightforward.

The equation for the estimation in the process shown in Figure 46 is,

$$
\text { Estimated velocity }=\frac{[\text { Final Position }- \text { Initial Position }]}{\text { Time elapsed }}(\text { while in Feedback })
$$

Next we will describe the frequency modulation technique in more detail. 


\section{CHAPTER 5}

\section{FREQUENCY MODULATION in the WGAS-SANM QTF-SPM}

In terms of its scientific application in scanning probe microscopy (SPM) systems, frequency modulation (FM) decouples the probe's interaction forces (for appropriate quality factor $(Q)$ values and initial phase settings[110]). In terms of SPM control, FM can be described as a proportional-integral (PI) loop embedded within another to control the phase between QTF's driving voltage and measured voltage response. Lastly, in terms of the mathematical analysis of the single harmonic oscillator, FM provides an

extra condition $\left(90^{\circ}\right.$ out of phase with the driving signal or $x=x_{0} e^{-i \frac{\pi}{2}} e^{i \omega_{0} t}$ as we saw in chapter 2) which produces the two equations for calculating $k_{\text {int }}$ and $b_{\text {int }}$ of the probe's motion (Appendix A).

This chapter takes a closer look at the FM's technical details. The core of a FM system (as well as of lock-in amplifiers) is the phase-lock loop (PLL). The PLL is the general name for a circuit that demodulates an oscillating signal of initial frequency $f_{M}$ by 'locking' its phase to the reference phase of another signal from an internal generator (called Voltage Controlled Oscillator or VCO). When this happens the phase between both signals remains constant. The output of the PLL is proportional to this constant phase (in the form of a DC voltage), which also turns out to be proportional to the frequency difference because is directly driving the VCO. In fact it is proportional to $\Delta f=f_{M}-f_{0}$, where $f_{0}$ is the center frequency of the VCO. 


\subsection{Frequency modulation}

In a lock-in amplifier, the PLL tracks the external reference frequency fed into the lock-in. Lock-in amplifiers are widely used in amplitude modulation SPM (AM-SPM) configurations.

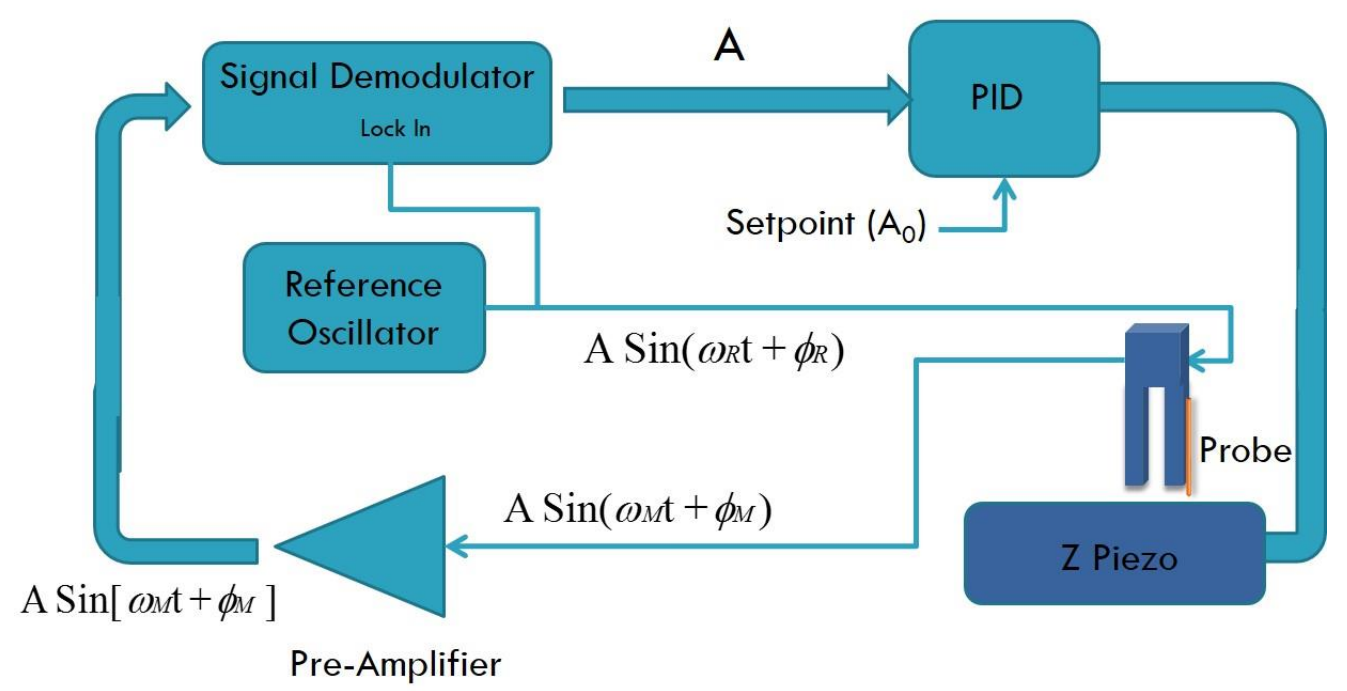

Figure 47. Schematic of an amplitude modulated SPM. There is only one feedback loop (thick arrows), which is used to control the probe-sample distance. PID stands for proportional-integral-differential, although in SPM application the differential gain is set to zero resulting in only PI control.

In traditional AM-SPM the signal coming from the QTF $A \operatorname{Sin}\left(\omega_{M} t+\phi_{M}\right)$ is analyzed with a lock-in amplifier which provides the amplitude $R$, that in turn feeds the PI stage. The excitation voltage $A \operatorname{Sin}\left(\omega_{R} t+\phi_{R}\right)$ driving the QTF in this case is constant in frequency and amplitude and serves both to drive the QTF and as a reference to the signal demodulator (SD). 


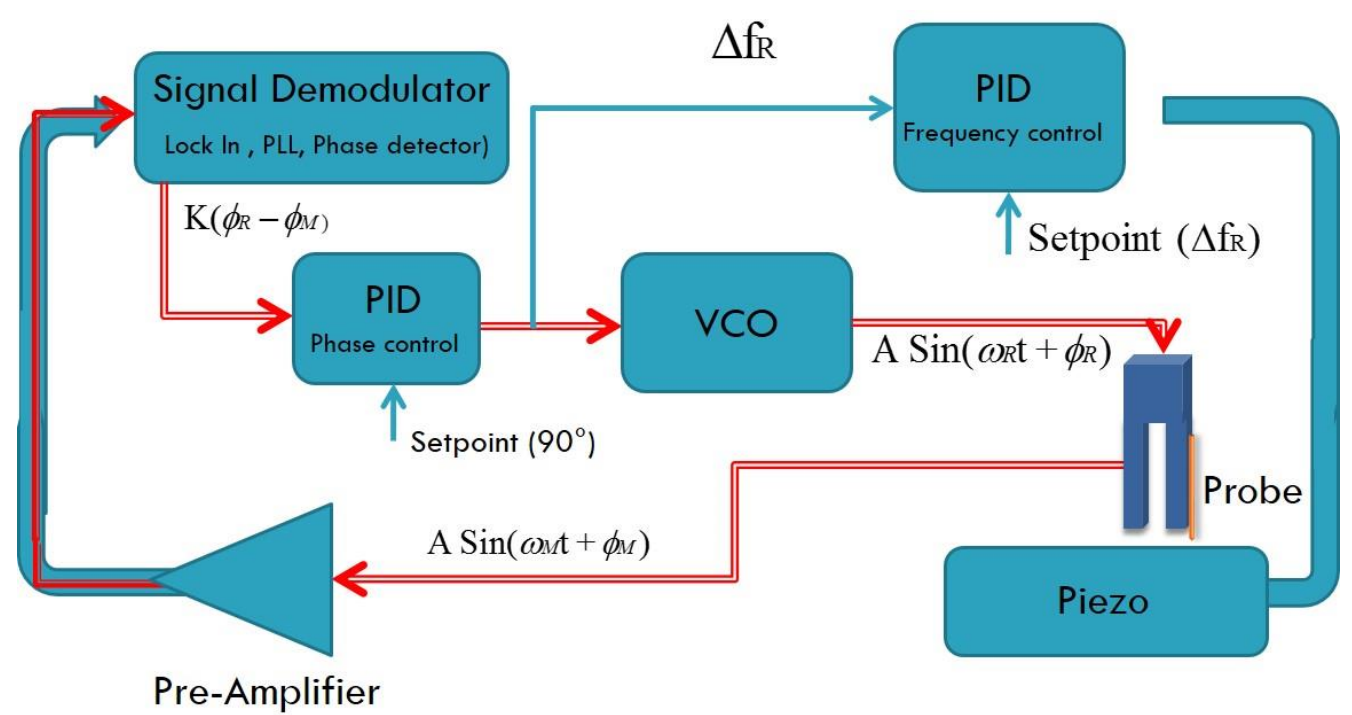

Figure 48. The new feedback loop for FM-SPM includes a feedback inner loop (double line arrows) to keep the phase constant at 90 degrees.

A frequency modulation SPM (FM-SPM) configuration is obtained by adding a feedback mechanism between the SD (lock-in, PLL or phase detector) and the QTF excitation signal. A phase difference $\left(\phi_{M}-\phi_{R}\right)$ is introduced between the excitation signal and the measured signal as variable to be controlled, by adding an embedded feedback loop which sole purpose is to keep that difference constant at 90 degrees. The new setup (Figure 48) of the FM-SPM will still include the SD, only this time the phase difference will be used. The purpose of the new embedded feedback (double line arrows) is to keep the phase set to a constant value (90 degrees). The signal coming from the QTF probe $A \operatorname{Sin}\left(\omega_{M} t+\phi_{M}\right)$ is processed by the signal demodulator. Depending on the phase detector of the signal demodulator (SD) the output could be proportional to the phase $K \phi$ or proportional to $K \operatorname{Sin}(\phi)$ when the signal is in lock. For 
simplicity here we use the $K \phi$ as the output. This signal is now the feed into the phase control PID that will try to keep it as close as it can to a setpoint value (90 degrees). In doing so, the input to the phase control loop VCO will be proportional to the frequency shift necessary to keep the phase at that value. This signal is the $\Delta f_{R}$ that will feed the external PID loop, called here Frequency Control PID.

In this thesis we use the standalone Nanonis PLL module: the OC-4 system. A more accurate FM description of this system can be drafted by using block diagrams. WE first start with the block contained in the OC-4 module.

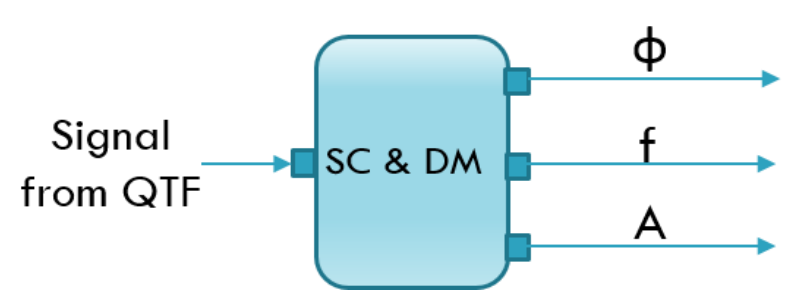

Figure 49. The signal demodulating block. It is physically located inside the OC-4 system. An additional external pre-amplifier was included between the QTF and the SC \& DM input (section 3.2.1).

A first block is used to demodulate the signal measured from the QTF, called here the signal conditioning and demodulation module (SC\&DM). It amplifies and filters the QTF's signal, process it and it outputs signal's main parameters, namely phase, frequency and amplitude. Another important block inside the OC-4 is the signal generating block (VCO DDS). It stands for the voltage control oscillator discussed above. 
However, current systems use digital counterparts called direct digital synthesizers (DDS) blocks. We include both terms to clarify its origin. The VCO DDS receives the demodulated information from the SC\&DM block and generates the signal used for both the QTF's excitation, and for external synchronization.

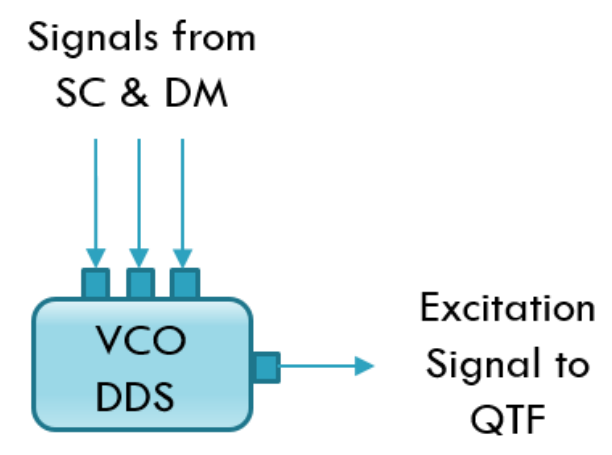

Figure 50. Signal generating block (voltage control oscillator). It drives the frequency changes when the system is in frequency modulation (FM) mode.

The final part contained in the OC- 4 is the PID module that process the signal and responds to maintain the control parameter (phase for FM) close to a predetermined value (not shown). Its output is the part of the loop that controls the tip sample distance by sending a signal to the piezoelectric positioning system. The output is called the 'control signal'. The complete SPM diagram is shown in Figure 52. The diagram also includes the user interface (UI) and the data acquisition (DA) modules. The thick lines show the SPM control feedback loop path. The green thick arrow shows the path of the main (only) loop for the amplitude modulation scheme. The UI and DA 
blocks are I/O channels for parameters and data transfer, but do not intervene in the system's real time control.

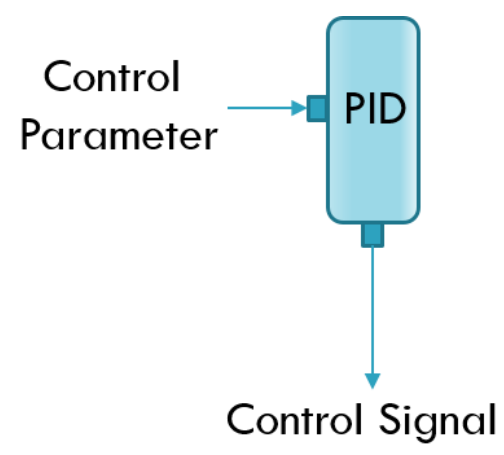

Figure 51. Simple PID block receiving control parameter for the probesample distance feedback.

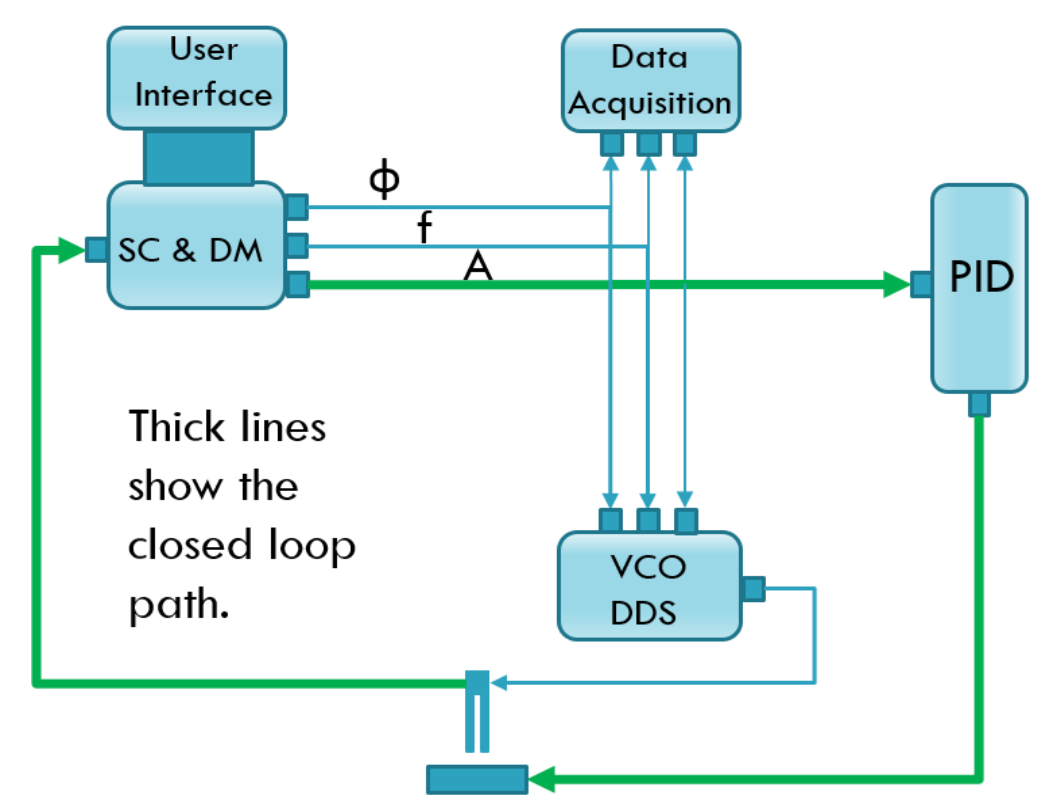

\section{Amplitude Modulation SPM}

Figure 52. Block diagram of the SPM amplitude modulation (AM-SPM). Notice a single PID loop, the QTF probe-sample distance control. 
Frequency modulation is a modification of the system described above with the purpose of increase the control over the parameters of the probe's motion. In the AM scheme the amplitude of the QTF is passively measured to feed the feedback system. In contrast the FM scheme seeks to control the oscillation of the probe itself by adding PID loops to the excitation loop that has remained open in the AM diagram. By adding a PID block in between the SC\&DM block the closed loop for the QTF excitation is implemented. Figure 53 shows in dashed lines the path that corresponds to the excitation closed loop.

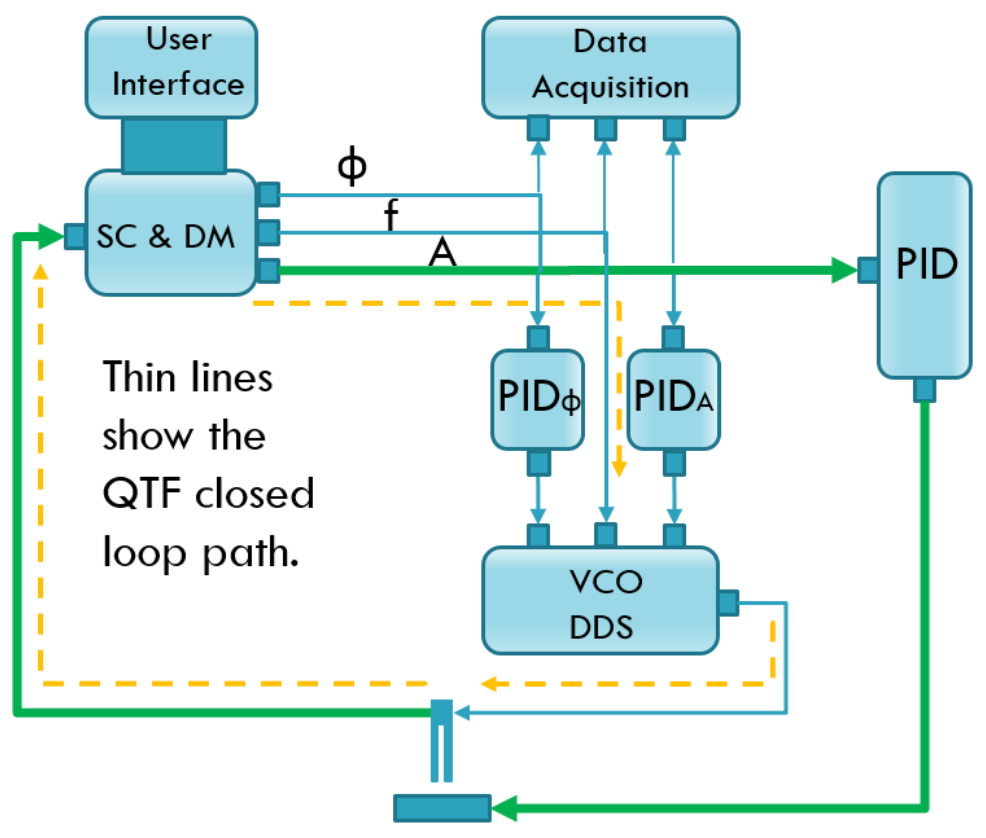

Frequency Modulation SPM

Figure 53. The dashed line shows the addition of an inner PID loop that takes care of the QTF excitation. As opposed to AM scheme the FM scheme actively controls the QTF's amplitude, phase and frequency. 
The same route could be taken with the phase $(\phi)$ signal. Tracing the loop starting at the phase output from the SC \& DM instead of the amplitude (A) output. In this new configuration the phase and $\phi$ and A signals are kept 'constant' (around a fixed value, with their stability depending on their particular PID parameters). This is rather valuable and its importance should not be overlooked. In physics, a theory has greater value when it can express several independent variables related through a constant (energy conservation, angular momentum conservation). By adding the ability to keep the $\phi$ and $A$ signals almost constant (or within a small value range) we can interpret the results through more simplified equations. As explained before, when the phase difference between the input (excitation) signal and the output signal is kept at 90 degrees, the system is in natural resonance. As a result we can keep the excitation of the QTF at its resonant frequency, within certain limits, as will be seen in chapter 6 .

\subsection{Frequency shift as control signal}

The frequency shift has showed unmatched reliability as a signal control provided the QTF, with the attached probe, has a high Q value and a carefully tuned PID values for the phase control. It is used as the control signal in all of the experiments performed in this thesis.

In order to quantify the time response of the proposed FM-WGAS, a tip was brought into the interaction region of a clean silicon sample, until the amplitude reached $50 \%$ of its maximum amplitude. A sudden upwards step motion of the tip was 
performed ('tip position' curve in Figure 55) with the tip still remaining inside the water layer.

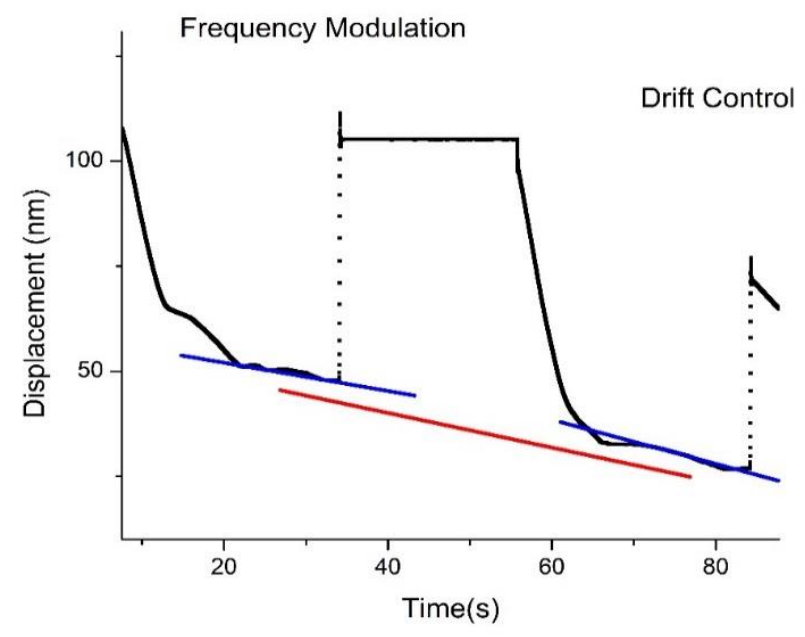

Figure 54. Drift experimental evaluation using FM mode with the frequency shift as the control signal. The two upper lines (blue) are the drift slope while in feedback and the lower line (red) is the average calculated by the ARCP.

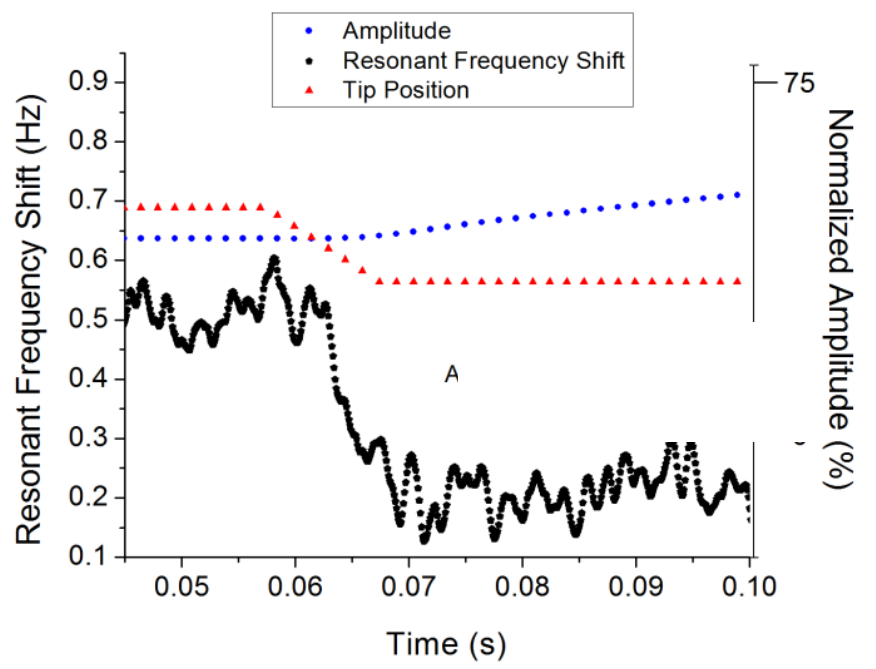

Figure 55. Step motion inside the interaction region showing the difference in time response between FM and AM. 
The corresponding change in amplitude (from $50 \%$ to $70 \%$ of its signal), frequency shift, and position were recorded simultaneously. The time response of the amplitude signal (not shown) is $85 \mathrm{~ms}$ and the one from the frequency shift signal is 2.5 ms.
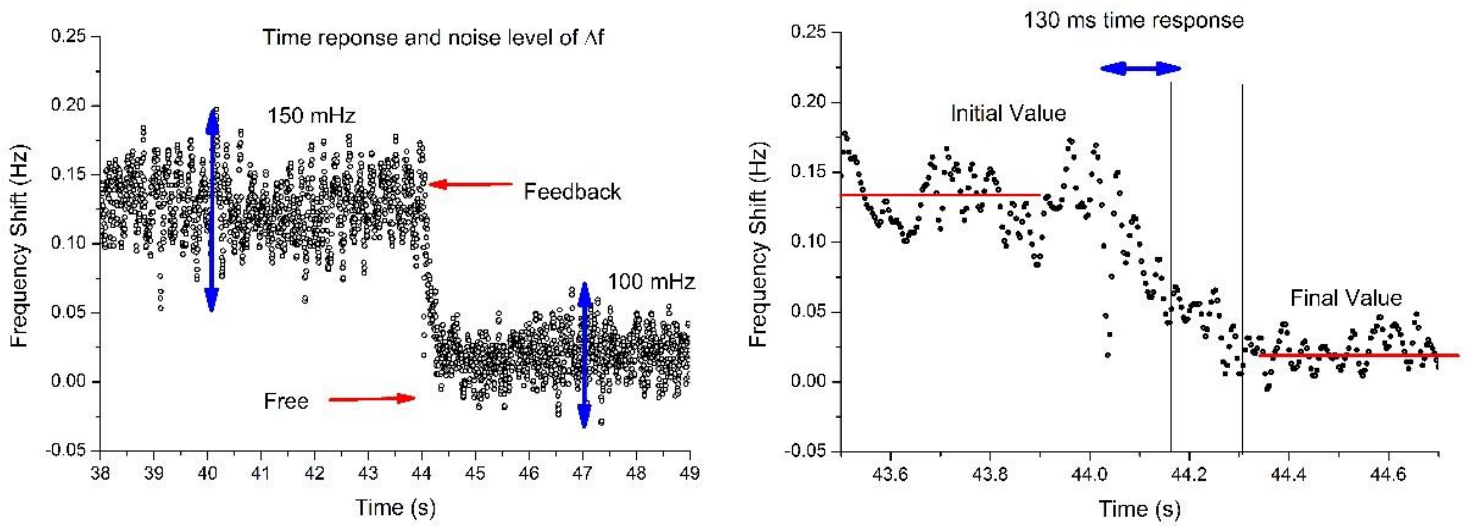

Figure 56. Time response of the frequency shift used in the approaching and retracting curves (ARC). The time constant has been increased in order to improve SNR of the frequency shift signal.

\subsection{Acoustic sensor to determine the real amplitude variation}

QTF sensors, as discussed in chapter 1, produce two signals. One caused by the oscillation of the piezoelectric nature of the quartz and the other by a capacitance signal originating in the separation between electrodes and electrical connectors. Capacitance compensation circuits have been used[76] to cancel the extra signal that distorts the frequency response and complicates the evaluation of the oscillation amplitude. 
Here we used the oscillations on the metallic SPM head which act as a cavity, and the oscillations are proportional to oscillations of the QTF. In this manner, while in constant amplitude mode we were able to detect the real change in amplitude despite the efforts of the OC-4. This was due to the fact that the impedance increases with frequency and thus its contribution to the total signal varies, causing a effective decrease of the amplitude. This is shown in Figure 57.

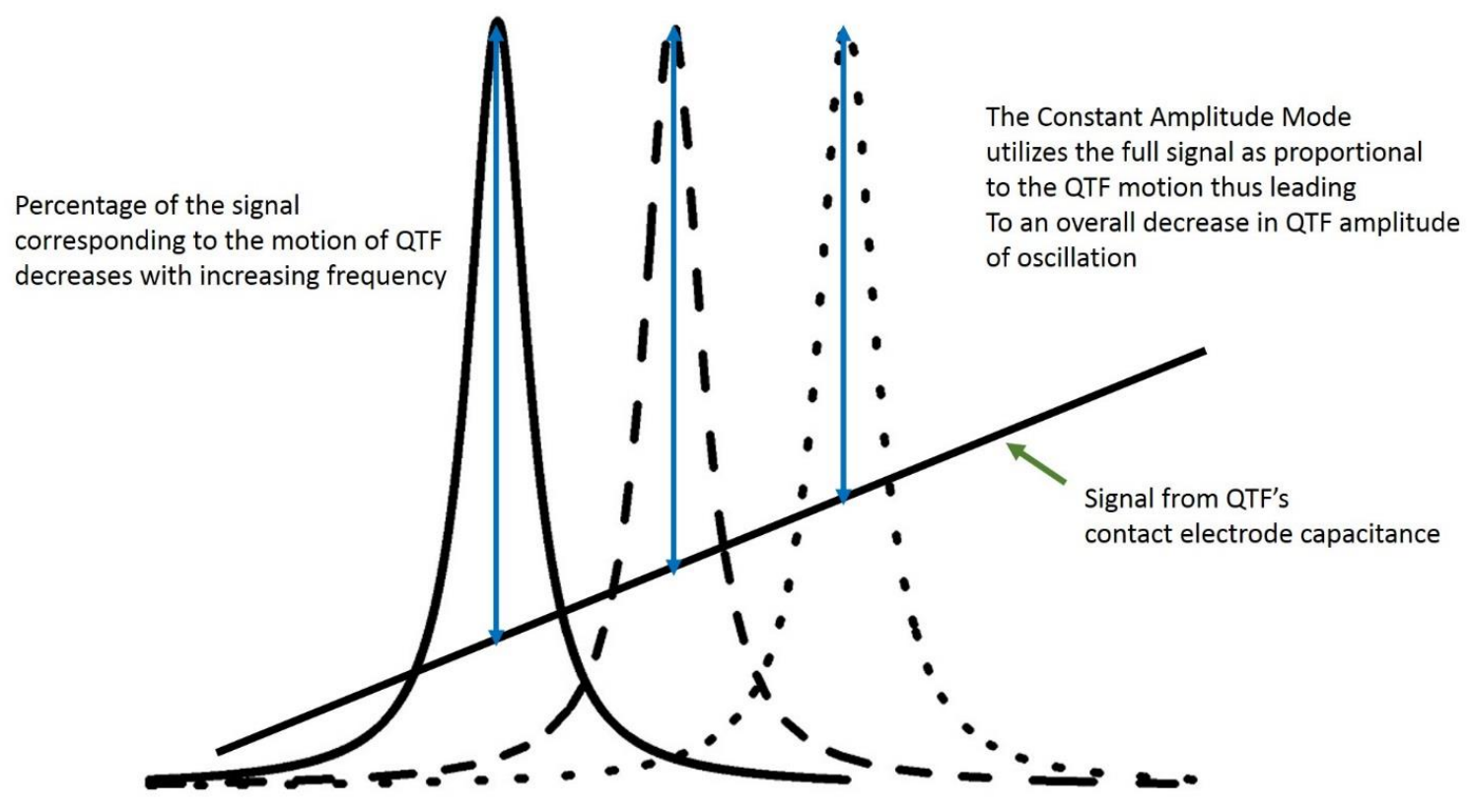

Figure 57. The change in the contribution of the contact capacitance and the RLC circuit to the QTF signal. As the resonant frequency of the system shifts to the right, the capacitance increases its contribution to the measured signal. 


\section{CHAPTER 6}

\section{CHARACTERIZATION of MESOSCOPIC FLUID-LIKE FILMS UNDER CONFINEMENT. APPROACH AND RETRACTION CURVES PERFORMED on HOPG, MICA and HYDROGEN TERMINATED SILICON.}

Approaching and retracting curves (ARC) record the interactions affecting the probe during its journey towards the sample. As discussed in previous sections, by simultaneously measuring the acoustic and electrical response of the probe-sample contact we gather multiple signals looking for cross-correlations in the different regimes of the confinement process. In this context, the tunneling current is used to narrow the region where the mechanical contact between the probe and sample occurs.

In chapter 2 we learned that the ARCP performs the approach until it reaches a predetermined setpoint of the control signal. Systematically approaching the probe towards the sample in successive steps, each one deeper than the previous (i.e. first approaching until the control signal decreases by, for example, $10 \%$ and retract immediately; subsequently approaching until the signal decreases by $20 \%$, etc.) we assure the tip wetting process is smooth and incremental, leading to stable curves. Increasing the driving force improves $\mathrm{S} / \mathrm{N}$ ratio but also increases the area of interaction and layer disturbance. The SNR for the QTF is $54 \mathrm{~dB}$ and $40 \mathrm{~dB}$ for the WGAS signal.

From the curve's results, the layer seems to act as a dissipation channel, with the contact induced meniscus formation increasing the coupling between probe and 
sample. In addition, the first set of curves in Mica and HOPG consistently display different features than subsequent ones. One such feature is that in the first approaches the sudden and small jump in the shear force when contacting the adsorbed layer leads to no signal of the SANM. An explanation may lie in the slow time response of the mesoscopic fluid layer adsorbed to the substrate which is unable to form a meniscus around the probe (increasing the mechanical coupling). In addition after the first approaches, there is material transfer between the layer adsorbed to the sample and the and the one on the surface of the probe. The increase affinity between both surfaces may lead to better conditions for the generation of sound.

The dissipation and elastic shear forces are calculated from the WGAS signal unless otherwise specified. Similarly, every current measurement is expressed in nA. All signals are locked to the driving signal of the QTF, with the driving frequency at any given time is $f_{0}=\Delta f_{0}+f_{00}$, with $f_{00}$ being the frequency with the QTF away from the adsorbed layer, or center frequency. The lock-in detection for every measurement has a time constant of $0.2 \mathrm{~ms}(777 \mathrm{~Hz}$ cut off frequency).

The position of the contact with the sample is not yet entirely clear. Despite having strong indications given primarily by the tunneling current and correlations with other signals, a thorough explanation is not yet available due to the observed complex behavior that further experiments need to address. 


\subsection{Highly Ordered Pyrolitic Graphite (HOPG)}

\subsection{A Sample Preparation}

A SPI-2 grade $7 \mathrm{~mm} \times 7 \mathrm{~mm} \times 1 \mathrm{~mm}$ HOPG sample from SPI supplies (equivalent to the ZYB grade from other manufacturers) was secured with carbon conductive tape to a 10 $\mathrm{cm}$ silicon square taken from a $150 \mathrm{~mm}$ silicon wafer. Thin copper wires were used for connection to a bracket with BNC connectors placed near the SPM stage. From there, BNC coaxial cables connected the sample to the SR570 from Stanford Research for amplification and subsequently to the SR 850 lock-in amplifier from Stanford Research.

The sample was cleaved with scotch tape until the area of the flake removed in one cleave covered at least half of the HOPG square, ensuring a large flat and clean area. The SPM head was mounted immediately afterwards, placing the probe right above the flat area on the HOPG. This procedure takes between 20 to 40 minutes and the coarse approach process that brings the probe tens of nanometers form the interfacial layer, takes between 60 to 90 minutes at a velocity of $4-5 \mathrm{~nm} / \mathrm{s}$. The ARCs take an average of 2 minutes to perform, including the drift calculation, depending on the layer thickness and the quality of the control signal. 


\subsection{B Probing in the low interaction forces regime (up to $5 \mathrm{nN}$ )}

The approach and retraction curves (ARC) performed on HOPG utilize the WGAS as the probe's oscillation amplitude control signal (for comparison purposes, the QTF signal is recorded as well). For the results reported in this section, the following parameters were used: $\mathrm{Q}=5,774$, oscillating amplitude of $26 \mathrm{~nm}$, center resonance frequency $f_{00}$ of $31804 \mathrm{~Hz}$, relative humidity $35 \pm 1 \%$ and a temperature of $26^{\circ}$ Celsius. The tip used for this process is shown in Figure 58. The driving force, calculated with equation ( 12 ), is $117 \mathrm{nN}$.

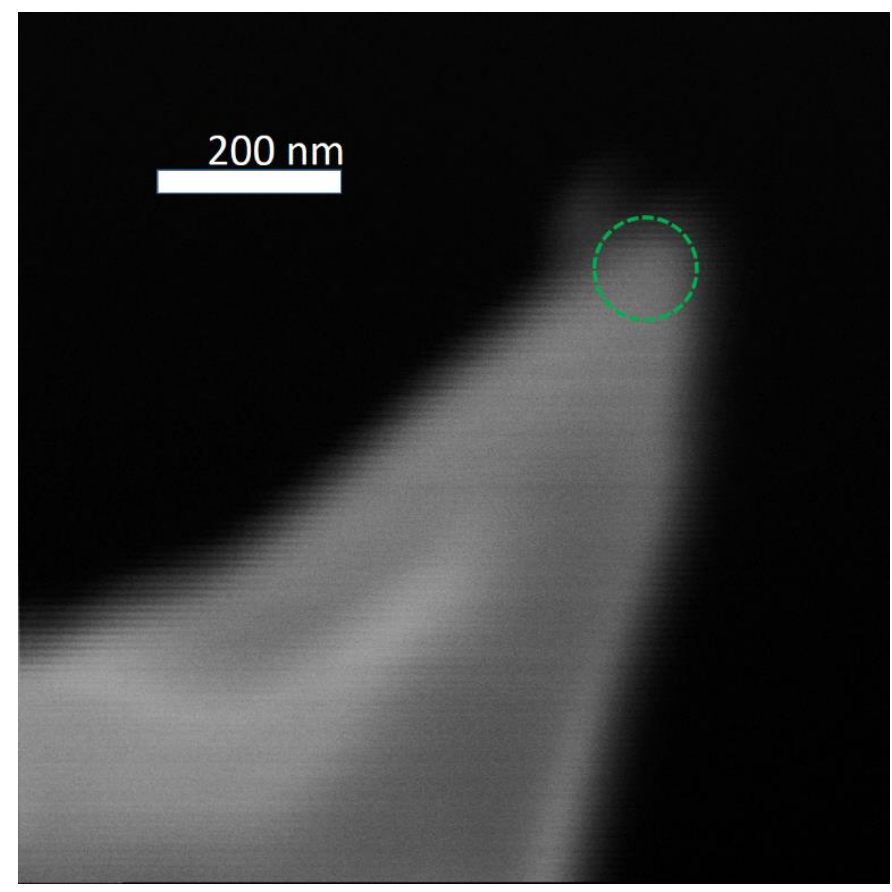

Figure 58. Tip used for the studies of the HOPG sample, interrogating the interaction forces in the lower range domain (below $2 \mathrm{nN}$ ). The apex has a radius of approximately $50 \mathrm{~nm}$. FE-SEM image taken using $10 \mathrm{kV}$ accelerating voltage and $5 \mu \mathrm{A}$ emission current. 


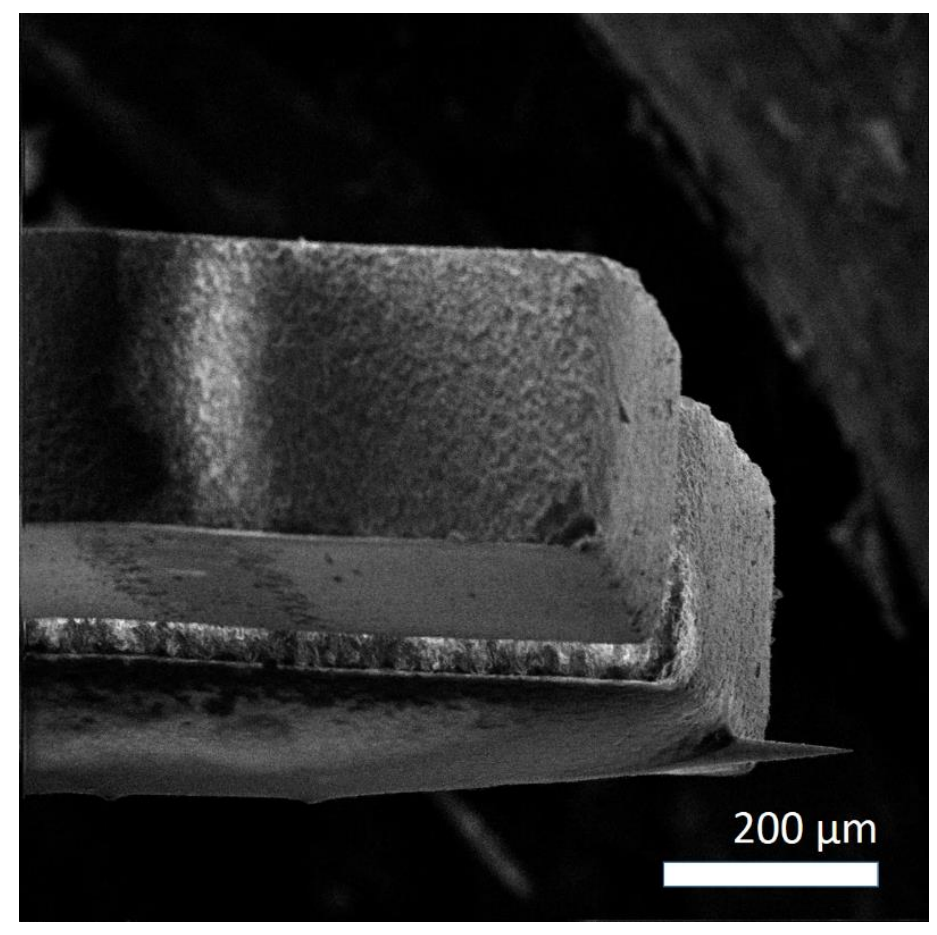

Figure 59. SEM image of the probe mounted on a QTF. The distance from the edge of the tine is $109 \mu \mathrm{m}$ and the radius at the base is $15 \mu \mathrm{m}$.

\subsection{Case: first approaches}

Figure 60 through Figure 64 show the different traces obtained during one of the first ARCs taken following the procedure outlined in the previous section. The $0 \AA$ marks the point at which the force was approximately $5 \mathrm{nN}$ and the ARC process stopped. From here on when discussing the location of the probe, we will follow the labels of the corresponding graphs, but it is assumed that the indicated positions are given relative to the end of the ARC process. In this approach curve, the elastic force, the tunneling 
current and the dissipation display a sudden change at the $100 \AA$ mark, the point intepreted as the location where the probe encounters the air-layer interface.
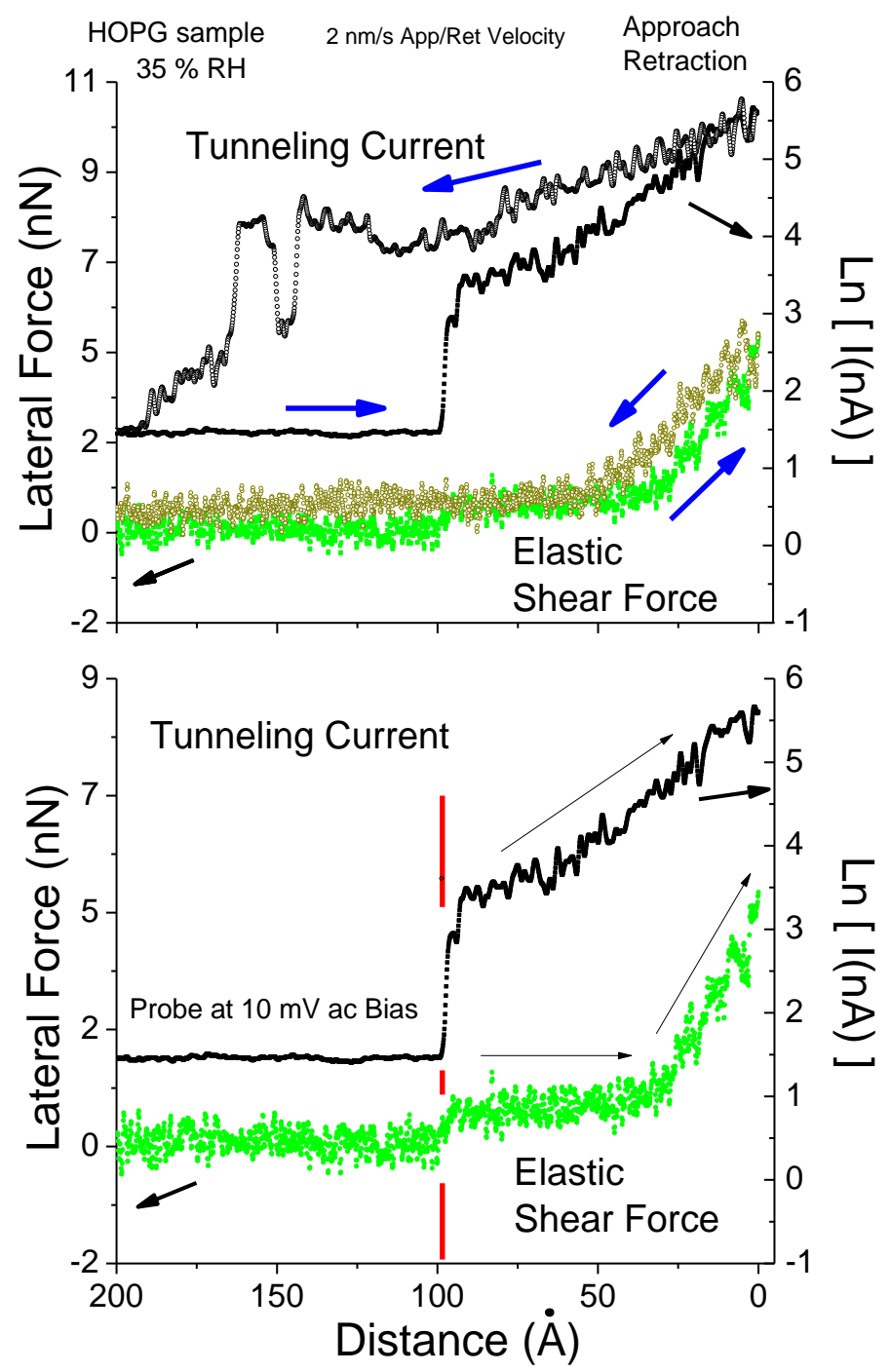

Figure 60. Top: Approaching and retracting curves of one of the first approaches on the HOPG surface, showing hysteresis. Bottom: Approach only. The vertical line around the $100 \AA$ mark indicates contact with the adsorbed layer. The tunneling current keeps increasing after the jump while the elastic shear force remains constant. The $0 \AA$ mark indicates the end of the ARC. 

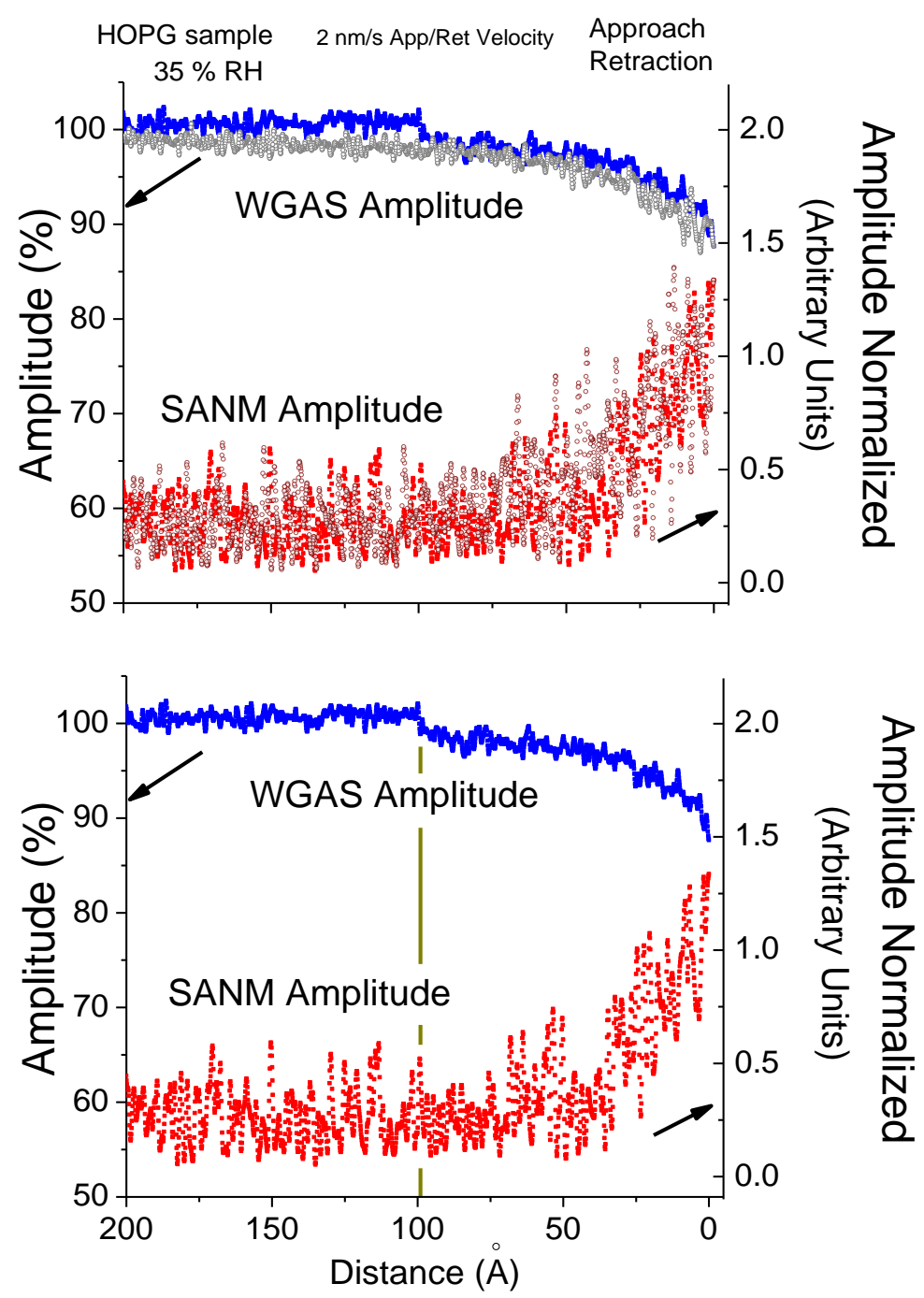

Figure 61. The WGAS amplitude, normalized in percentage value, and the normalized SANM amplitude. The normalization factor is the amplitude of oscillation of the QTF, i.e. the WGAS amplitude signal (see Figure 62 ). The vertical line marks the onset of probe-layer interaction.

Notice how all signals register the jump in interaction at the same position. According to previous reported experiments of STM at ambient conditions, this jump indicates initial contact with the layer [105], [106]. In the next $50 \AA$ the probe moves 104 
closer to the surface (Figure 60), the elastic force remains constant while the current increases. The elastic force peak to peak noise floor is about $0.3 \mathrm{nN}$. After remaining constant the elastic force starts to change linearly at the $30 \AA$ mark reaching $5 \mathrm{nN}$ at the end of the ARC. In the retracting curves (top section of Figure 60) the layer's viscosity and meniscus formation causes hysteresis, causing the elastic force to remain different from zero for another $100 \AA$ past the initial point of interfacial contact detected durgin the approach. Regarding the SANM amplitude, the normalization calculation is only relevant when there is confirmation of probe-sample coupling, which occurred in this case (Figure 62).

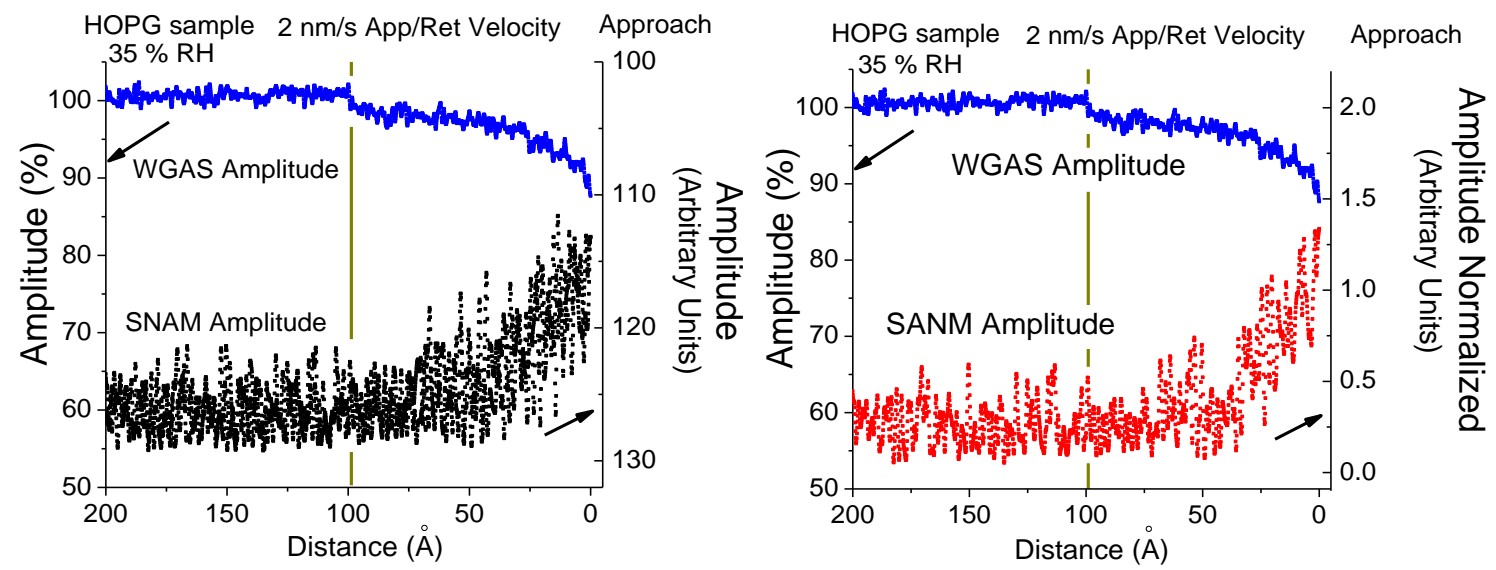

Figure 62. Comparison between the un-normalized (left) and normalized (right) SANM signals. Each data point on the normalized trace is obtained by dividing the un-normalized value by the corresponding probe's amplitude (the WGAS signal).

In this case, the normalization process allows comparing the strength of the acoustic signal engendered at different probe-sample distances by assuming they were 
caused by a constant QTF amplitude. Figure 62 shows both the normalized and unnormalized SANM amplitude. Figure 62 also shows the WGAS amplitude registering the jump when contacting the layer and the SANM amplitude seems to appear $50 \AA$ after the initial elastic shear force experienced a change as seen in Figure 60.
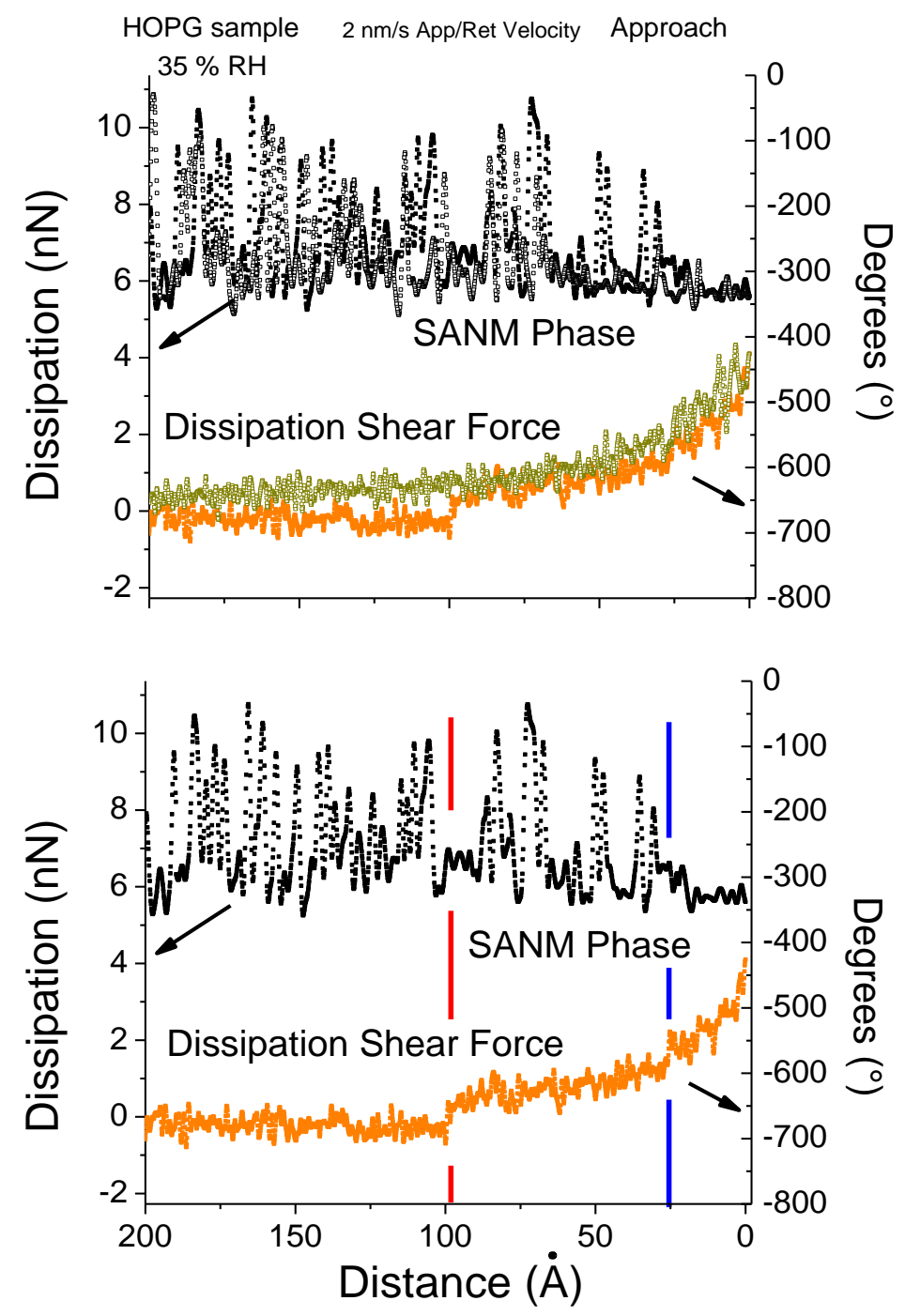

Figure 63. Top: ARC showing hysteresis of the dissipation force and SANM phase. Bottom: The vertical line, signals contact with the layer. 
The SANM phase is unstable, oscillating with an amplitude of almost $200^{\circ}$, before there is an elastic interaction with the probe as revealed in Figure 63. However, after contact with the layer the signal starts to settle, decreasing (lagging) to a value of about $-340^{\circ}$ (Figure 63).
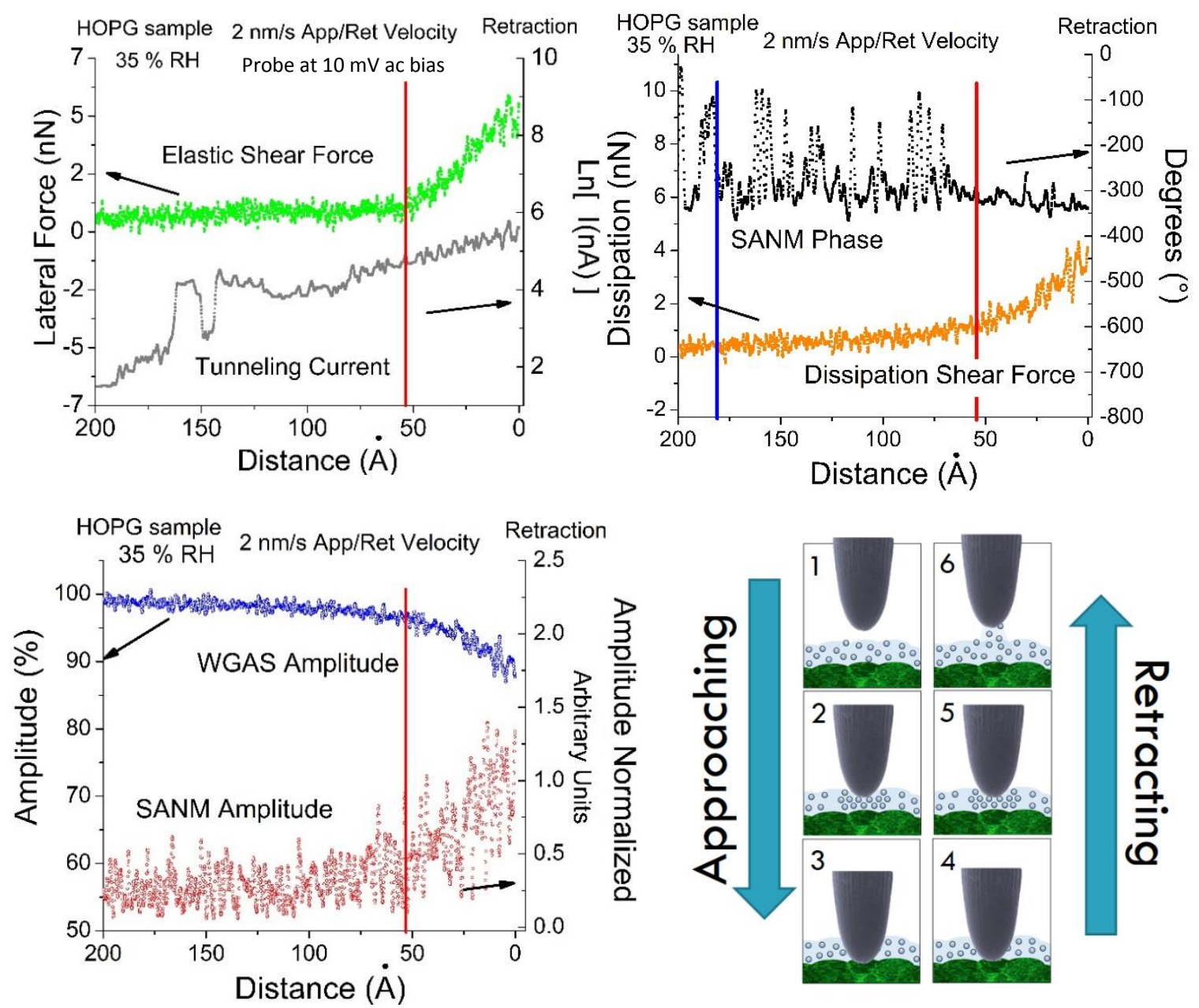

Figure 64. Retraction curves of all signals. The vertical line shows the change in the slope of the elastic force. However the change equivalent to the initial jump does not appear. This is considered evidence of viscous character of the adsorbed layer. The tunneling current is in nA. 
This is one of the features of the SANM integrated SPM used in this ARCs. The SANM phase is a measure of the relative phase between the adsorbed layer, and as the probe continues to approach the sample, the sample itself. The phase changes are related to dissipation channels[78], according to the single harmonic oscillator (SHO) model. Figure 63 shows the SANM phase signal along with the dissipation force.

It takes approximately $70 \AA$ after the interfacial contact for the SANM phase to reach a stable value. The dissipation force signal seems to slightly increase during the period after initial contact and follows an exponential behavior described in Karrai et al.[75].

While the elastic does not show a clear exponential behavior between $100 \AA$ and $30 \AA$ as the dissipation signal does (two well defined slopes, first flat and then linear) this behavior correlates well with the SANM amplitude. The results of the retraction curves show how the meniscus formation (Figure 64) causes a smooth transition when the probe leaves the adsorbed layer. The large discontinuity in the retracting tunneling current (around $150 \AA$ mark) is thought to be caused by the adsorbed material found on the probe in Figure 58. The elastic shear force reach a somewhat flat response after retracting to the $50 \AA$ mark. The dissipation signal, however, continues its seemingly exponential change until it has reached the $200 \AA$ mark.

Figure 65 shows the correlation between the frequency shift and both the elastic force and the SANM signal. 

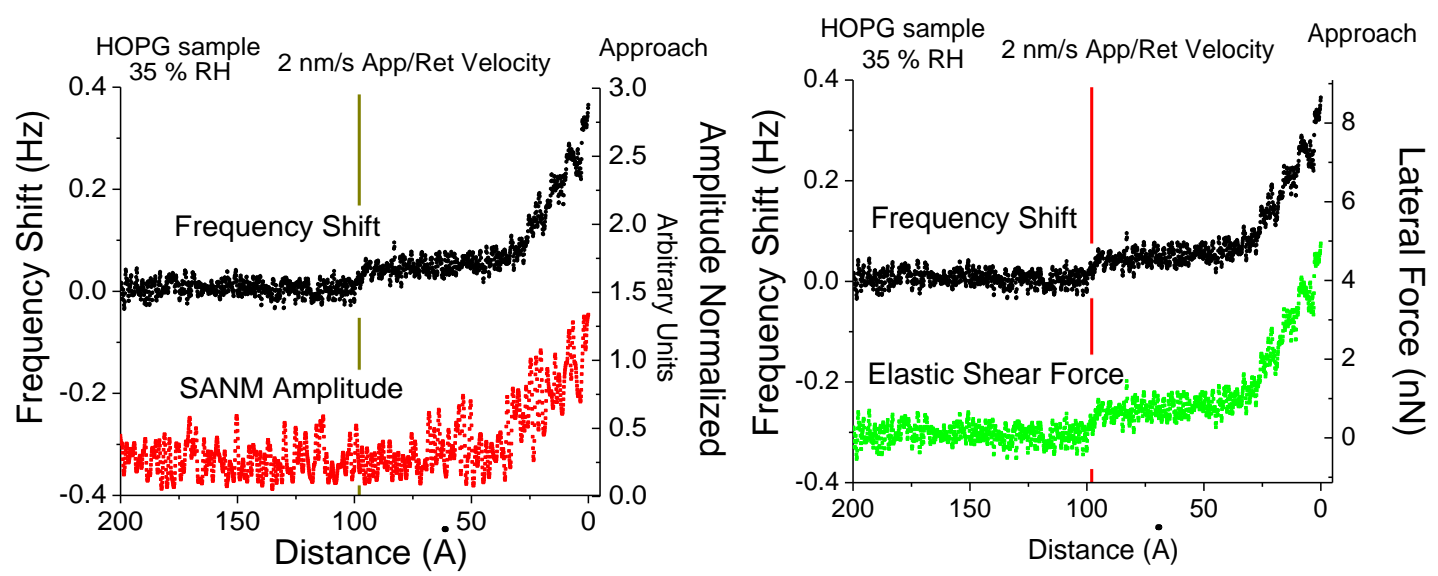

Figure 65. Left: Comparison between the SANM amplitude and the frequency shift for the first approach process obtained after peeling off the HOPG layer. Right: Comparison between the frequency shift of the WGAS signal and the elastic force.

The elastic force is calculated from the frequency shift with equation ( 8 ) and at low dissipation forces, the elastic force pattern is dominated by the frequency shift. In the next section we will analize the curves after the hysteresis becomes negligible.

\subsection{Case: subsequent approaches}

As the ARCs continue, the curves increase their reproducibility. Figure 66 shows this transition where, as opposed from the initial approaches, almost no hysteresis is observed. There is a subtle indication of hysteresis at the $40 \AA$ mark (from the arbitrary zero) in the case of the elastic force, however, which may indicate slight probe (or sample) deformation. Otherwise, the lack of hysteresis for the rest of the curve indicates an elastic process between probe and sample with no evidence of probe or surface plastic deformation involved in the ARC process at this time. 


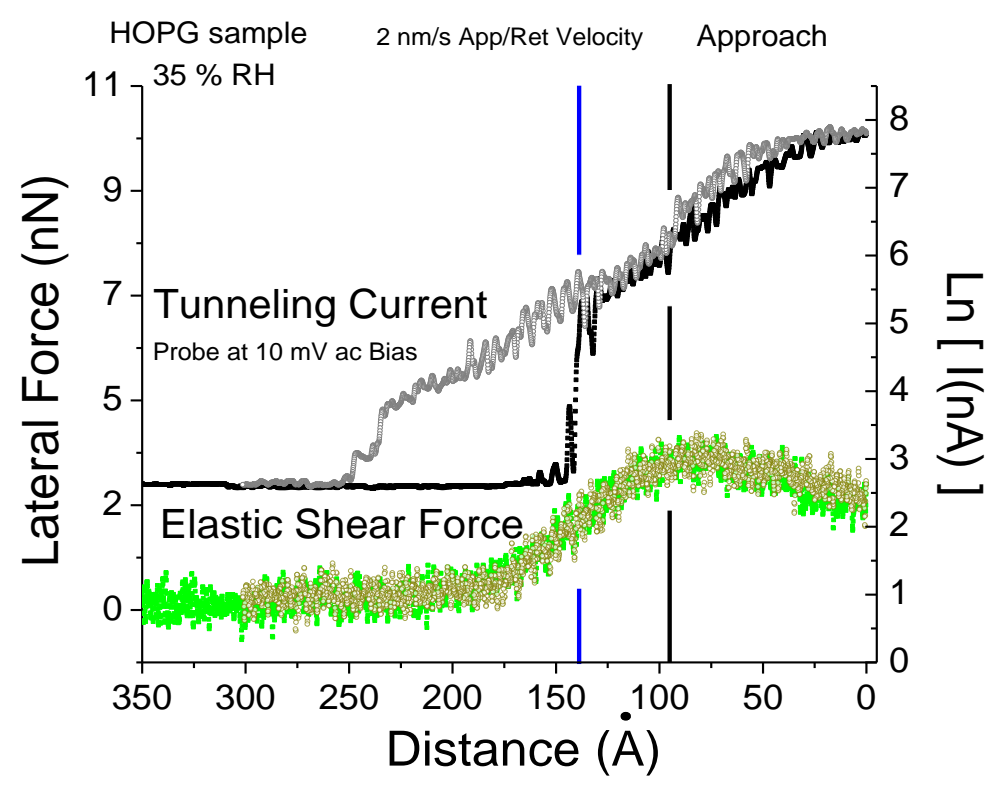

Figure 66. After several trials the ARCs start displaying no hysteresis in their interacting forces. The vertical line at around $150 \AA$ indicates interactions at the air-layer interface. Notice the existence of elastic force interaction $50 \AA$ before there is a tunneling signal. The vertical line around $90 \AA ̊$ indicates a transition to solid-like interactions.

Further, the interaction region is longer possibly caused by increased contact area brought about by probe deformation from previous ARCs which increases the distance from the sample at which the interaction starts[75]. Another important feature is the plateau observed in the tunneling current between the $50 \AA$ and the arbitrary zero. The decrease of the tunneling signal marks its saturation, and the cessation of its exponential trend. Thus the vertical line at approximately the $90 \AA$ mark is a strong indication of a different interaction medium, probably of probe sample-contact. 


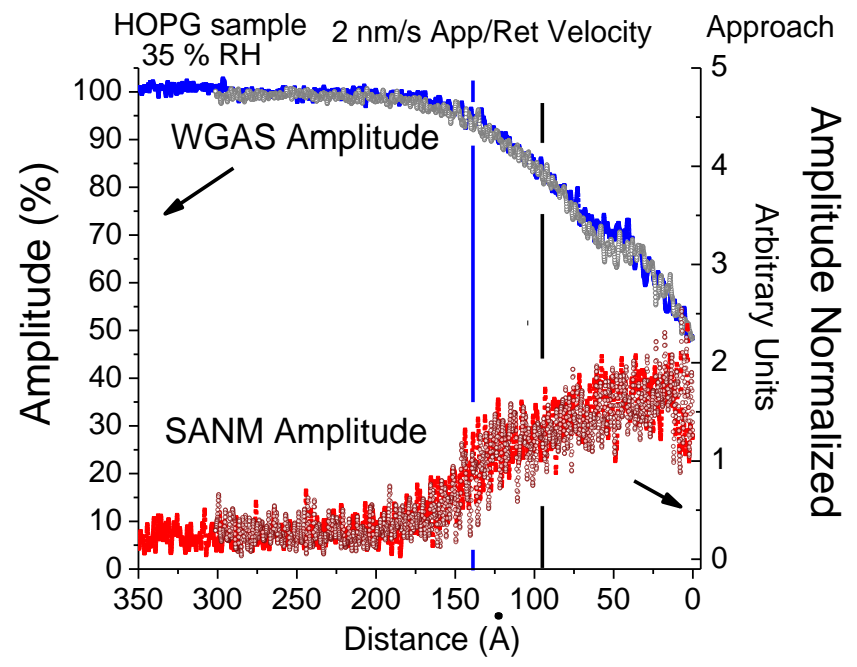

Figure 67. The SANM amplitude increases before the onset of tunneling current by approximately $30 \AA$. A correlation between this SANM amplitude and elastic force is observed.

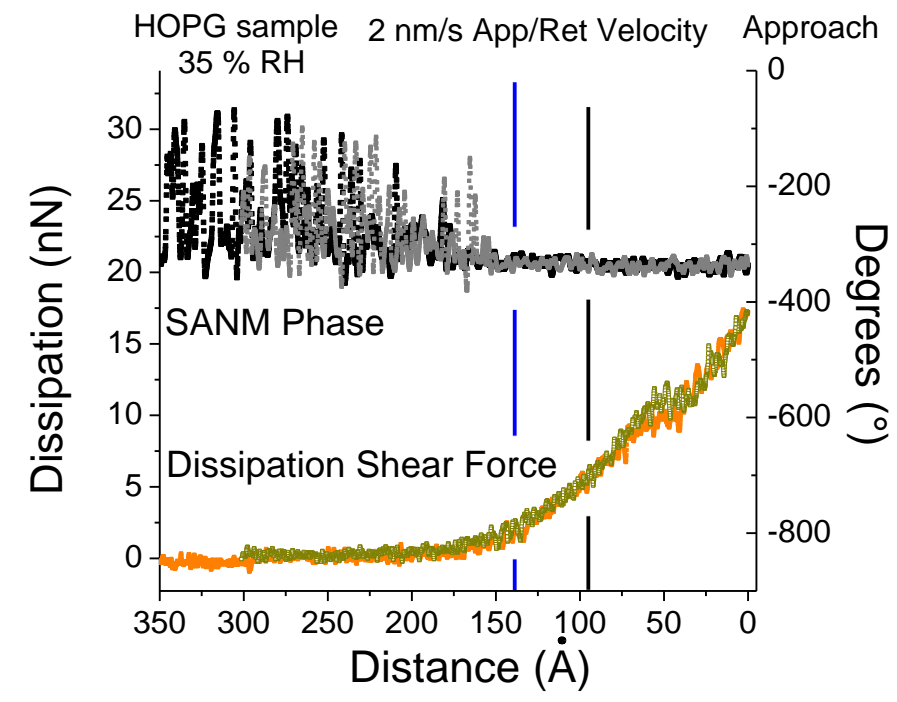

Figure 68. Dissipation force curve showing a marked increase in dissipation as the SANM phase becomes stable. The dissipation is now larger than the elastic force. A sign of medium change (or probe deformation) is seen around the $50 \AA$ mark of the dissipation curve. 
All signals, with the exception of the tunneling current (which starts its change at $140 \AA$ ) start to change their behavior around $180 \AA$ and then at around $100 \AA$ from the arbitrary zero. A third transition (more evident in the dissipation curve) occurs around $50 \AA$. One possibility is that the first transition indicates the layer contact, the second evidence of a change in the behavior of the layer from liquid-like to solid-like properties and the third represents probe-sample direct contact. We show next the analysis of the subsequent 4 curves.

\subsection{E Analysis of subsequent approaches.}

After performing several approaches the curves become very stable (i.e. become very similar among each other), which justifies to analyze their average response. The absence of hysteresis (Figure 69) which is evidence of a conservative (elastic) interaction, even in the event of contact with the surface it is evidence of elastic probe modification (bending). We take this behavior as indicating that the probe is mostly intact and no plastic deformation occurs, as opposed to later curves (not presented here) were the hysteresis is present and is attributed to plastic deformation due to an increased proba-sample contact area. 


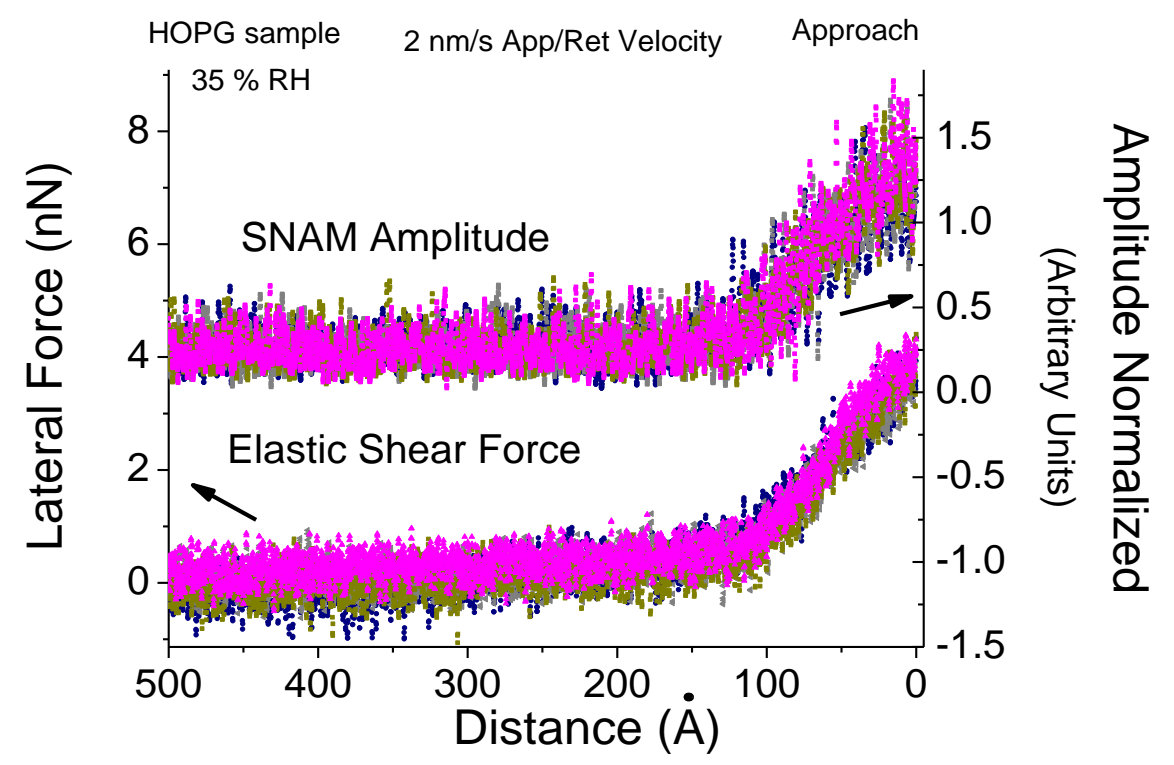

Figure 69. The ARCs show a repeatable pattern after severa cycles.

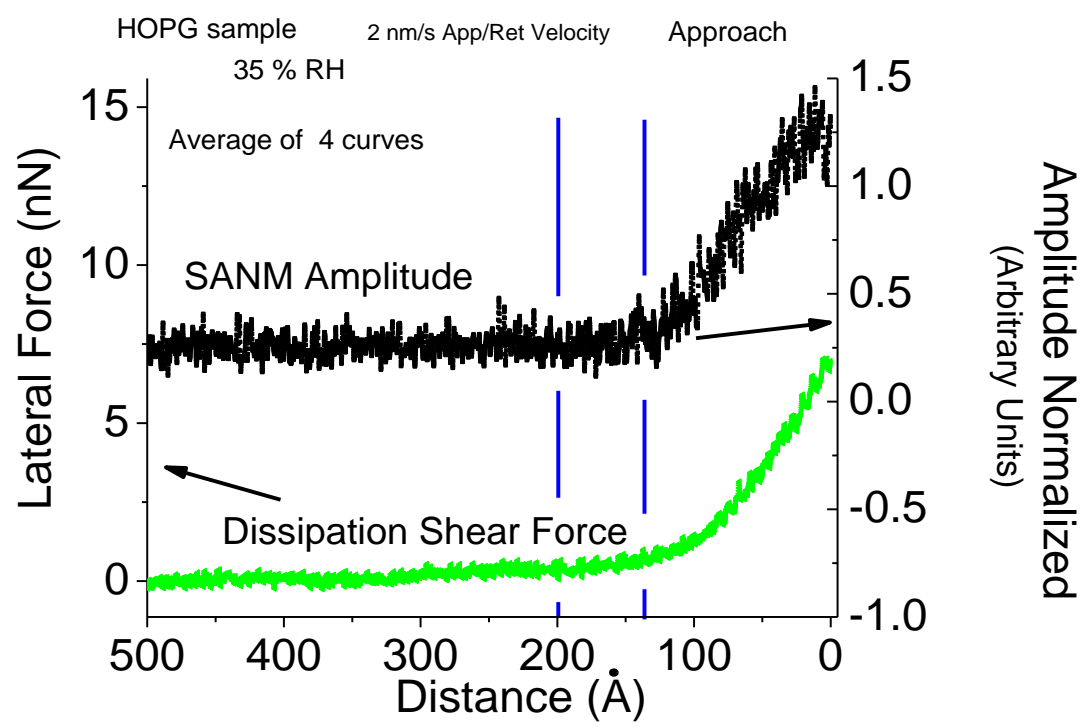

Figure 70. The SANM amplitude and the dissipation curve showing different inflexion regions. While the SANM amplitude displays a sharp change in the slope, the dissipation force has an exponential behavior (as will be seen in section 6.1.F) around the $130 \AA \AA$ mark. 


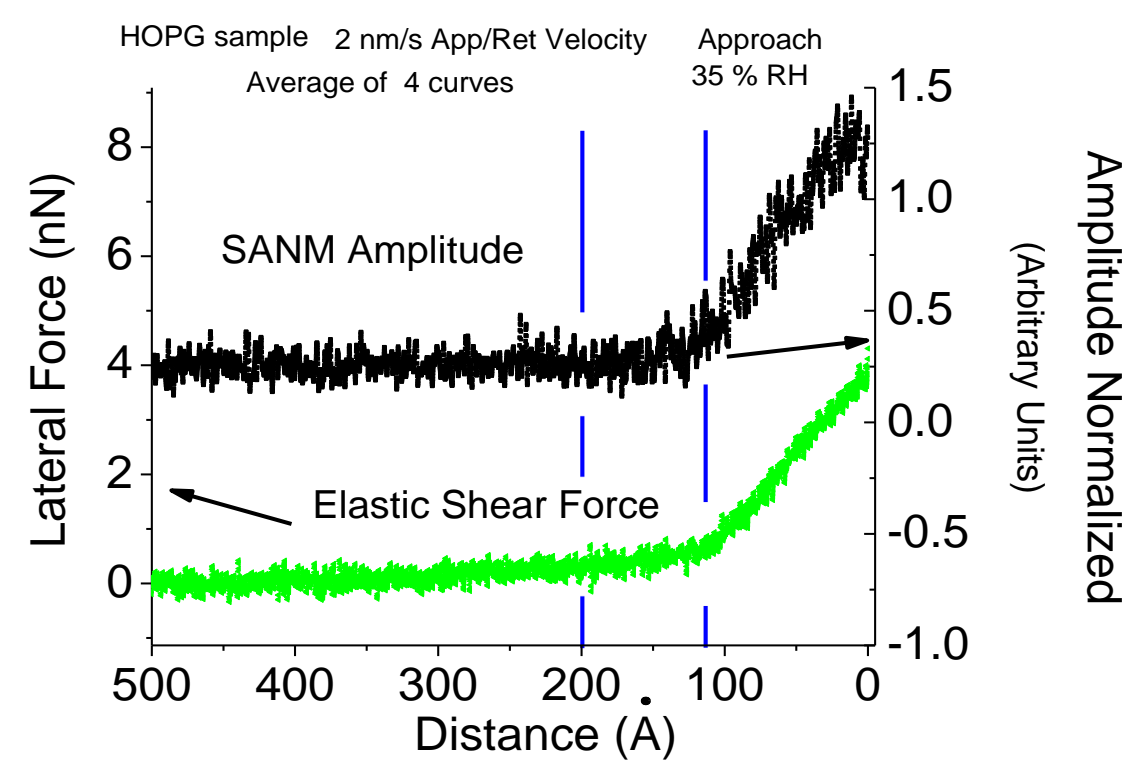

Figure 71. SANM amplitude and elastic force showing a remarkable correlation. Similar to the SANM (and contrary to the dissipation in Figure 70 ) the elastic curve has a sharp change: its slope is constant before and after the transition at around $130 \AA$.

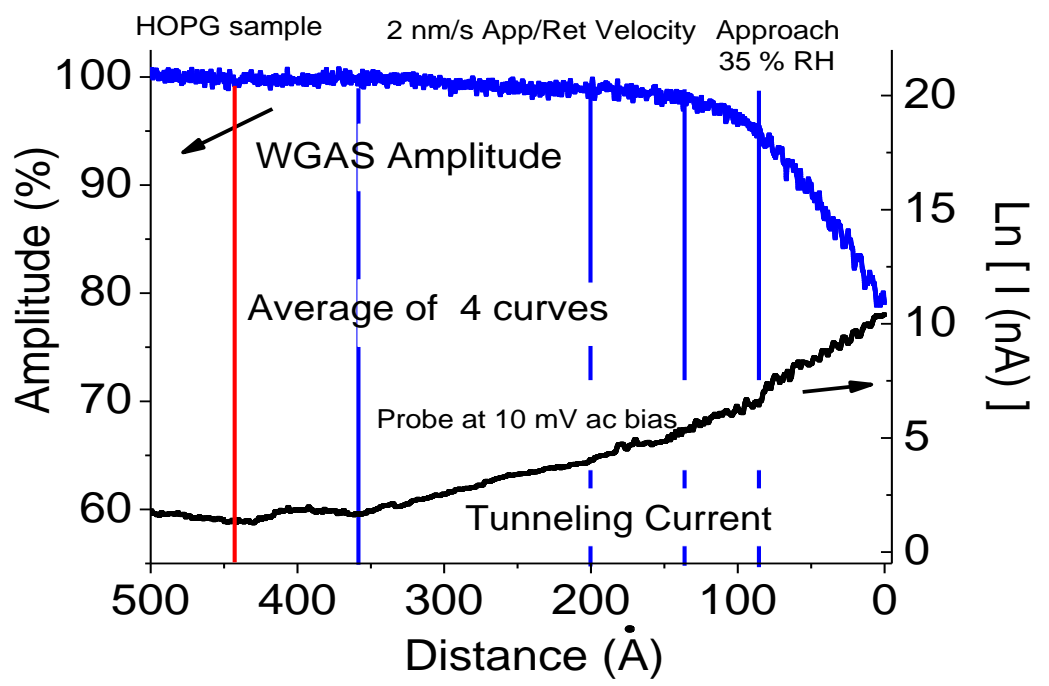

Figure 72. The WGAS amplitude shows a long interaction range of about 450 $\AA$ after several ARCs. The tunneling current does not present a clear air-layer transition, but its linear exponential behavior starts at the $360 \AA$ mark. The vertical lines mark different transition points for the tunneling current. 


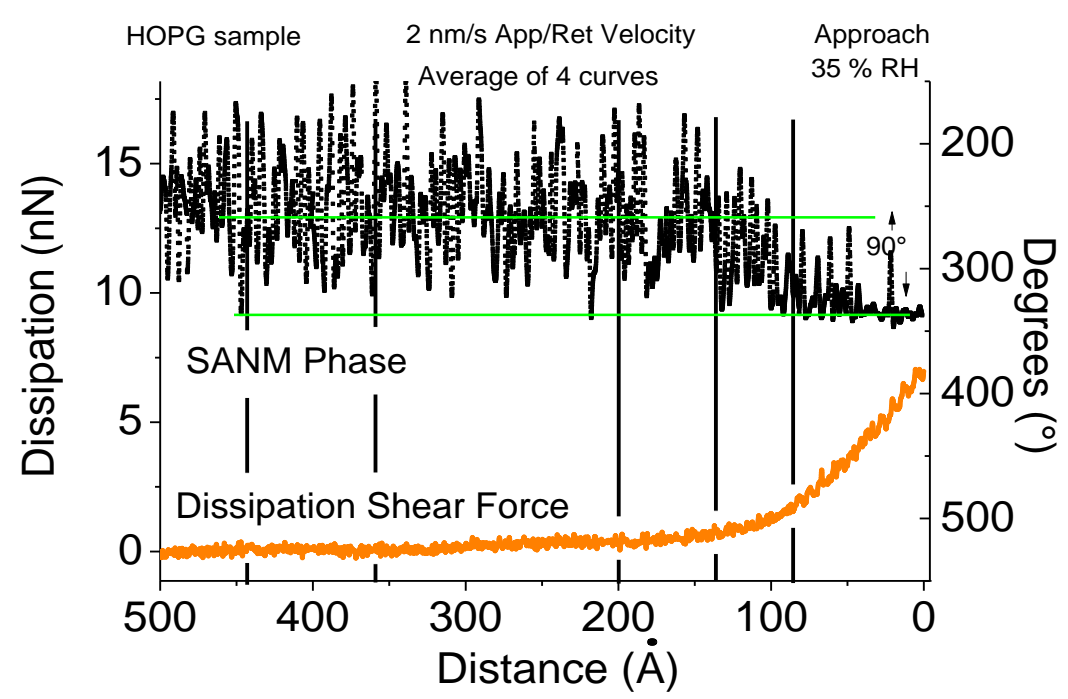

Figure 73. The SANM phase changes by approximately 90 degrees. The change correlates well with the start of the exponential behavior of the dissipation force. The vertical lines follow the transitions observed in the tunneling current (Figure 72) showing weakly (but existent) correlations.

The SANM phase shows a smooth transition from around the $140 \AA$ mark until the $50 \AA$ mark where it reaches a plateau. This coincides with the interaction transitions observed in the tunneling current curve and the dissipation force curves (Figure 72Figure 73). Relatively high currents (Figure 72) are unavoidable because the bias voltage is also the QTF excitation voltage and, since it determines the SNR of the control signal (frequency shift), it cannot be arbitrarily decreased. We will discuss this effect in more detail at the end of section 6.1.H. 


\subsection{F Viscous and elastic behavior}

It has been reported in the literature that the interaction elastic constant $k_{\text {eff }}$ of the QTF, when performing ARC on graphite, presents an exponential behavior when the assumed frictional drag force is proportional to velocity[75]. In this reported study[75], despite being performed in vaccum conditions $\left(6 \times 10^{-7}\right.$ Torr) it was concluded that adsorbed molecules may have remained on both the probe and sample's surfaces. In our results the first approach shows this exponential behavior, accounting for the viscous nature of the layer. Solid-solid interactions, however, will display a different behavior.
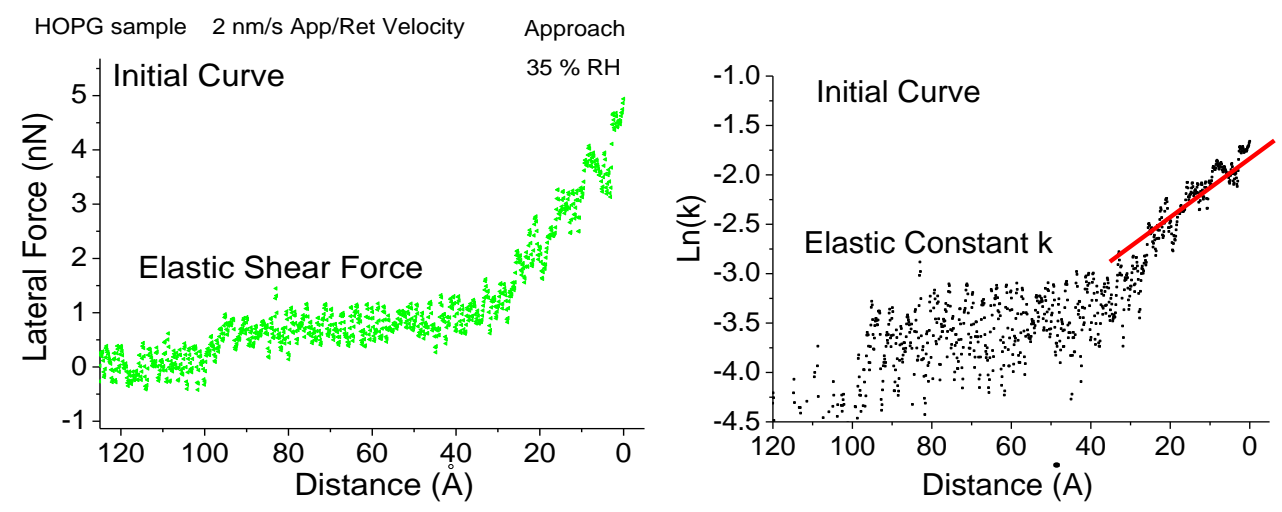

Figure 74. Comparison between the elastic force initial and subsequent curves. The force starts from longer distances as the number ARCs increases, in agreement with the higher interaction distances experienced with higher radii probes[75]. 

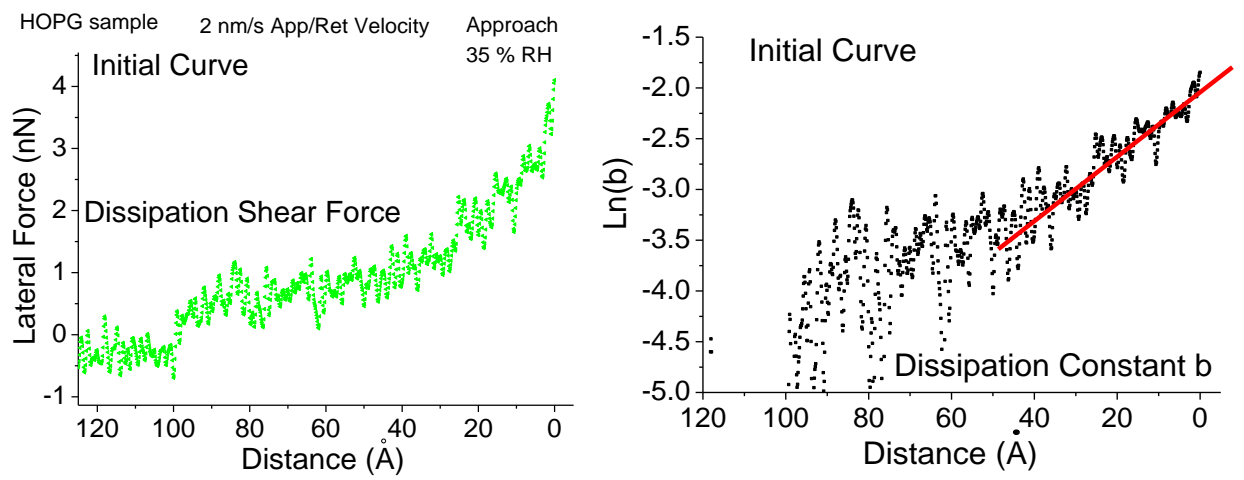

Figure 75. The same case occurs with the dissipation force. These curves present a higher curvature radius, when compared to the elastic force.

The friction force is proportional to the normal load, thus increasing linearly with distance in the absence of probe bending (compliance). Such a change in behavior provides then information necessary to compare the change in forces with the signal of the SANM. It has been argued[75] that the dominating drag force in this process is not a direct solid-solid contact nor a friction force that depends on the normal force. Our calculations seem to corroborate that claim. The slopes of the logharithm of the elastic force and dissipation force change indicate the characteristic behavior of elastic and dissipation forces measured in vaccum[75].

Figure 76 shows that the logarithm of the $k_{e f f}$ is linear from the initial contact up to $70 \AA$ into the adsorbed layer. After this mark the curve diverges and losses linearity. This behavior also appears in the logarithm of the dissipation constant (right). 

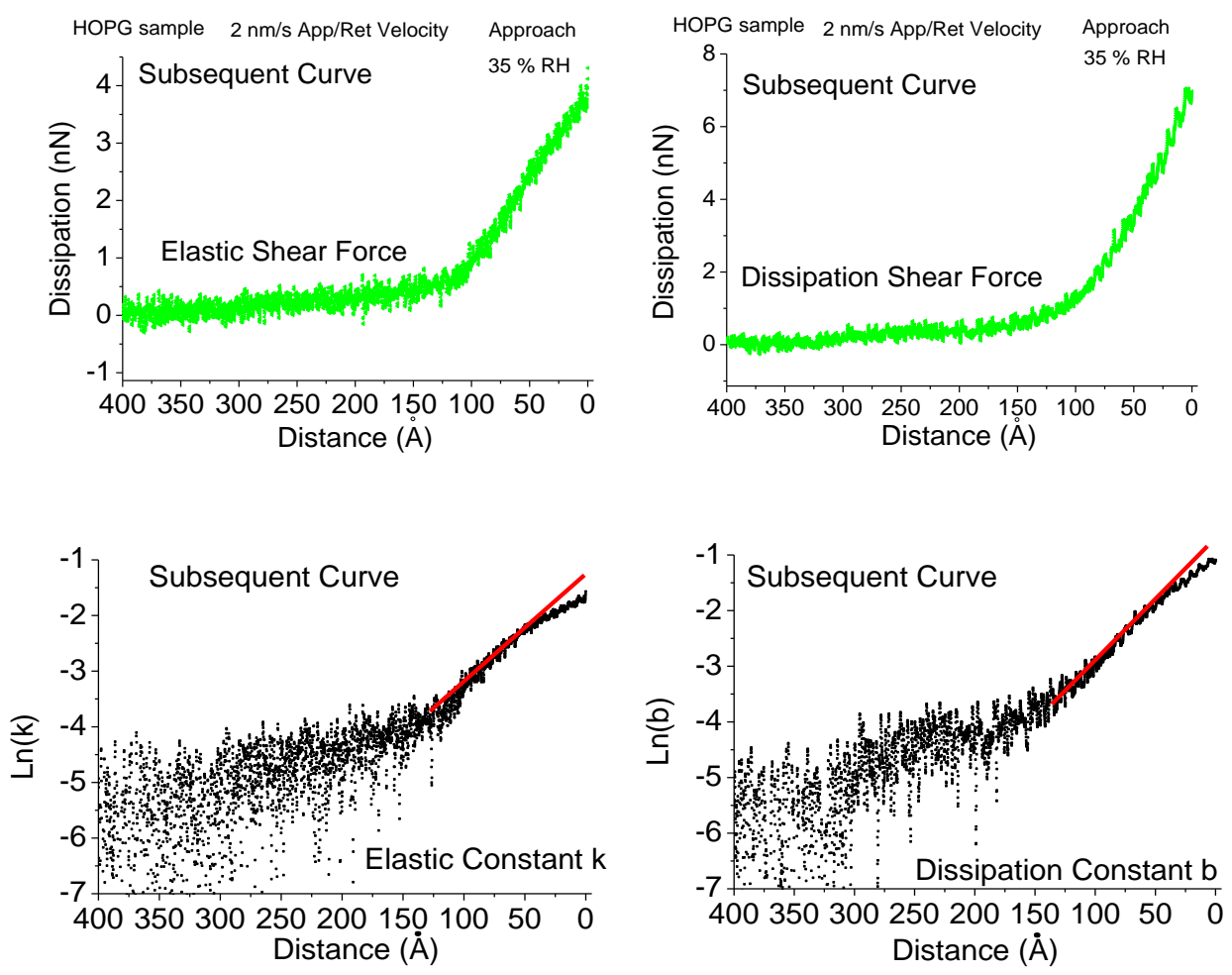

Figure 76. Top: Elastic and dissipation forces. Notice the difference in the inflexion. Bottom. Natural logarithm of the $k$ and $b$ for the subsequent curves. Both slops are constant for a short between $100 \AA$ and $50 \AA$. Below $50 \AA$, the behavior of both $k$ and $b$ is linear (and thus the natural logarithm arcs at that point). It is an indication of elastic contact between probe and sample as seen in nano-indentation processes.

\subsection{G Discussion about the phase of the SANM}

When the probe is still far from the sample the lock-in amplifier detects a background signal (generated by the QTF tines). This far away signal is smaller that what the probe-layer interaction is during the ARC, but is strong enough that the SANM phase is weakly locked with the phase of the motion of the QTF. Let us remember that at resonance the phase of the motion is at $90^{\circ}$ out of phase from the driving motion. Since 
the lock-in measuring the SANM signal is locked with the drving signal (in phase), the moment the signal from the near field interaction surpasses that of the far field, a transition should be observed with the SANM sensor, specifically the SANM phase.

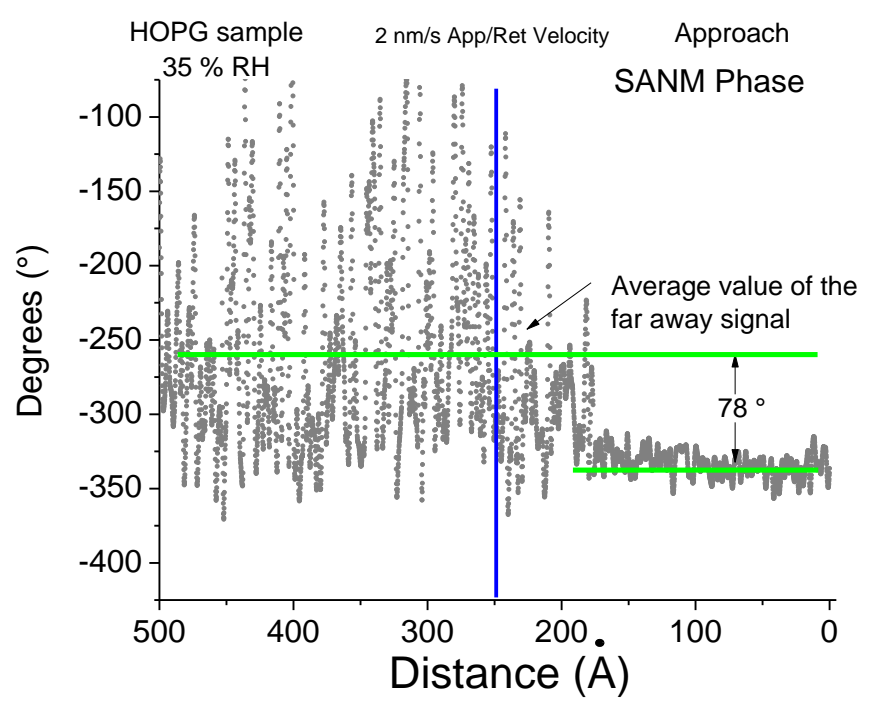

Figure 77. Change in phase from the SANM acoustic sensor at the onset of mechanical coupling from the probe. The noisy signal before interaction is due to the weakly locked phase between the SANM sensor and the QTF.

This is shown in Figure 77. The phase transition starts the moment the probe initiates its interaction with the layer. At this point SANM amplitude does not increase dramatically despite being in contact with the layer. The SANM amplitude increasea when the mechanical coupling is strong enough to act as a dissipation channel. 


\subsection{H Discussion about the SANM amplitude and the elastic shear force}

The correlation found between the SANM signal and the elastic force indicates a mechanical coupling through a mechanical channel proportional to the displacement (or and inertial drag force) and not the velocity which would imply a viscous nature.

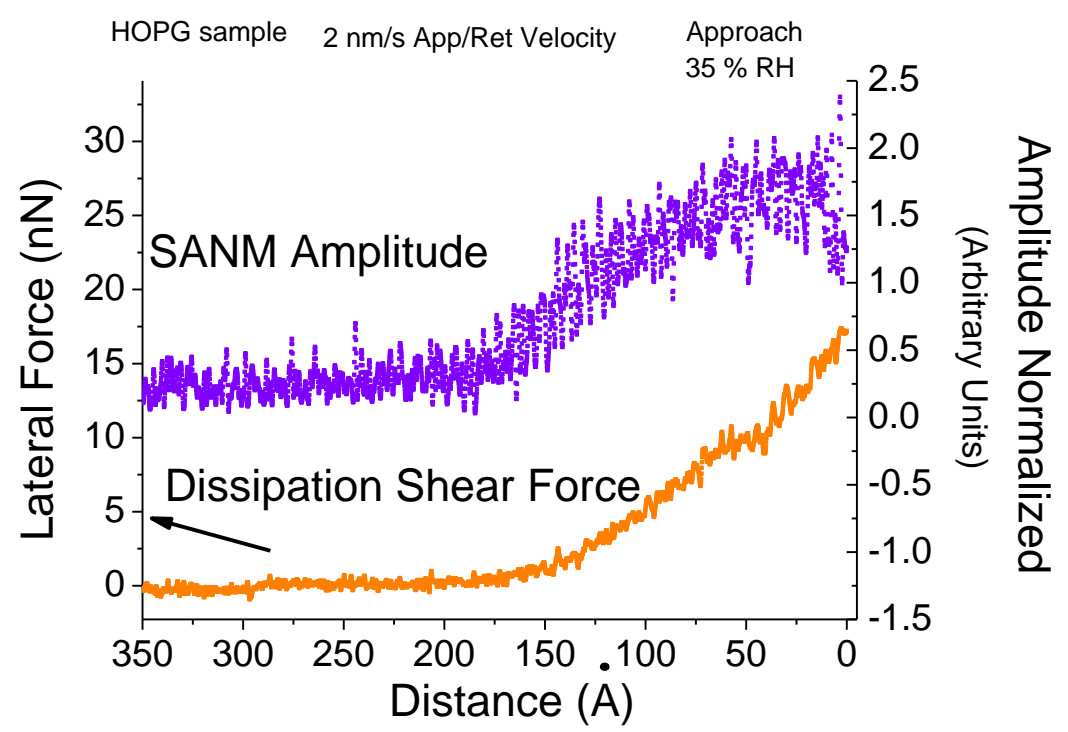

Figure 78. Comparison between the SANM amplitude and the dissipation force for the first process with no hysteresis (transition curve). The SANM and dissipation force signals show different trends.

The correlation between the frequency shift and the SANM amplitude is also worth mentioning since we directly measure the frequency shift and the elastic force is calculated afterwards. Figure 80 shows this correlation. The frequency shift, at low forces, determines the trend of the elastic force. 


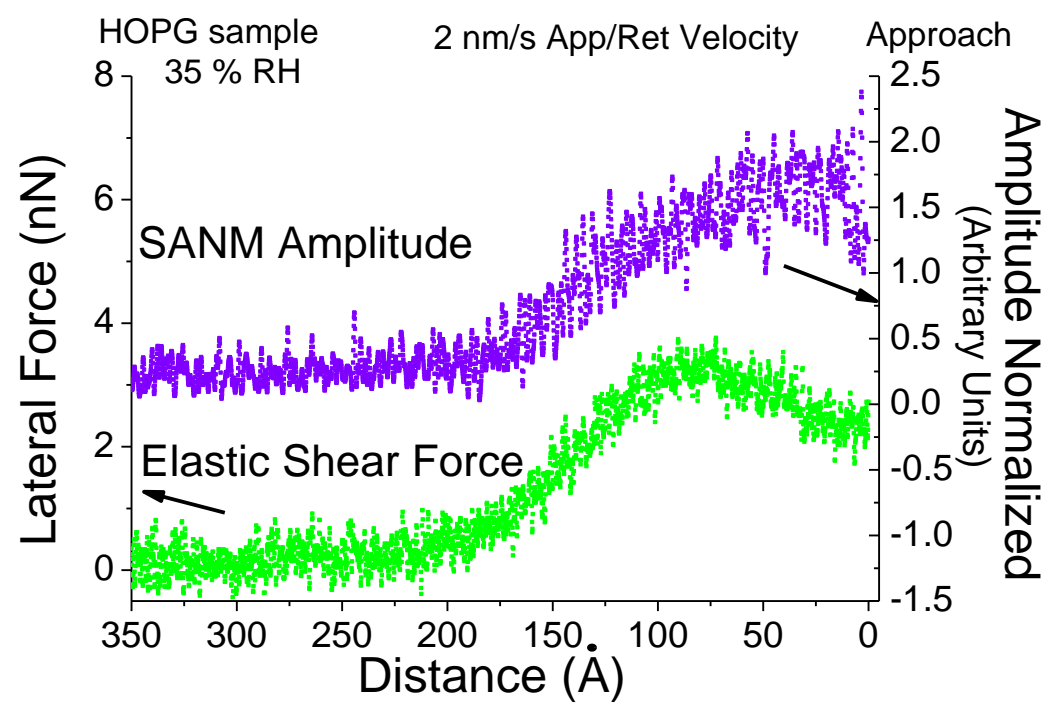

Figure 79. The correlation between the SANM amplitude and the elastic force of the transition curve.

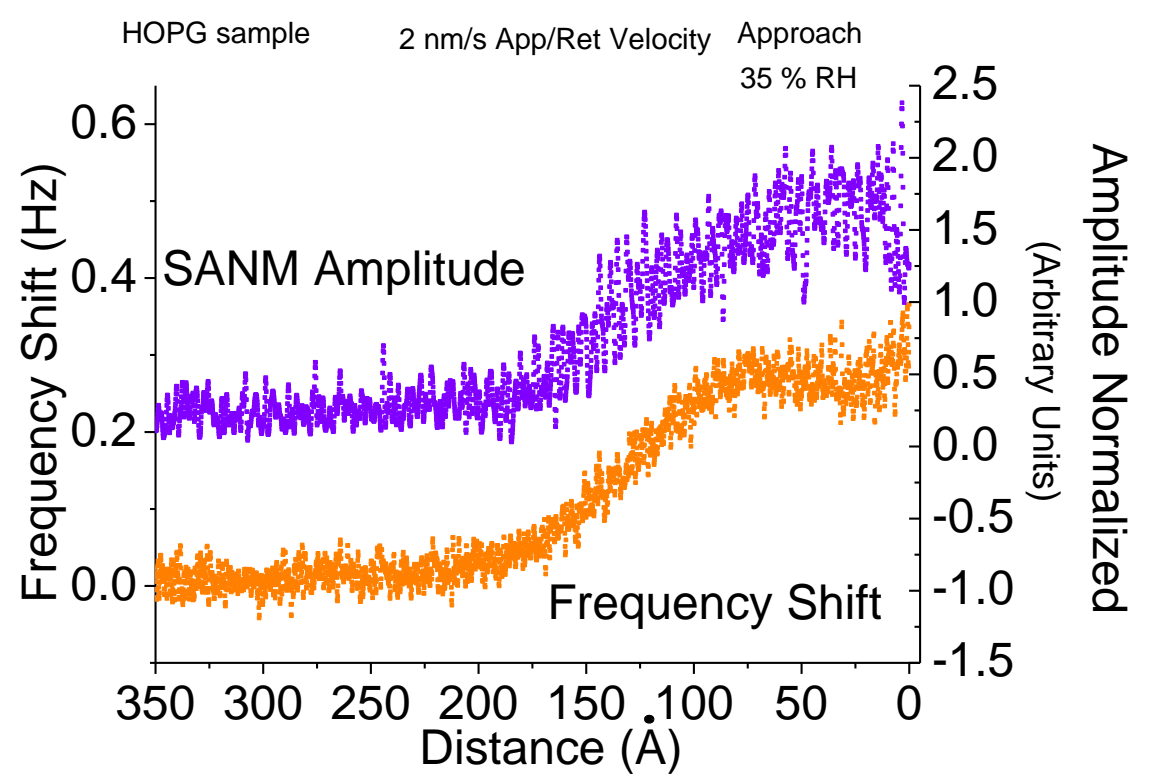

Figure 80. Comparison between SANM amplitude frequency shift in the transition curve (hysteresis becomes negligible). The rest of the curves for this process are show in Figure 66 through Figure 68. 
The tunneling current levels (200 nA - $1000 \mathrm{nA})$ used in the curves analyzed are similar to those used in the work of Hahn et al.[111]. However, the effects of hundreds of nanoamperes of current may contribute with forces caused by capacitance effects that have been linked to classical dipole induced forces[112]-[114], quantum effects of the sample's local density of states and the charge leakage by tunneling current in AC measurements[115]. Additionally, heating effects may contribute to deformation of the probe and contribute to the increase of the contact area. These forces may account for the deviations from the exponential trend shown in the analyzed curves.

The point at which this deformation starts to significantly affect the measurements is still not clear. We speculate this size effect starts to have a predominant role after the transition curves discussed above. Several curves taken after the curves analyzed here (we only present the first 10 curves from a total of a 100 taken with the same probe) showed huge hysteresis in their dissipation forces, hinting at a major change in the probe sample interaction that we associate with an enlarged contact area. Finally, in order to minimize these effects the next step would consist in decoupling the bias voltage of the tunneling current from the excitation of the QTF[116], both currently provided by the same contact point.

\subsection{Section conclusions}

The ARCs on freshly cleaved HOPG show multiple correlations between the SANM and WGAS signals. They point towards different interacting mediums (identified as specific 
slopes in the curves) as the probe approaches the surface. Indications of probe deformation were also observed, such as increased interaction distance and change in the exponential behavior of the dissipation force. Heating, capacitance and dipole effects due to the tunneling current may affect the interacting forces, but their contributions have not been yet determined. The SANM phase correlates very well with the transitions observed in the elastic and dissipation forces, presenting an in dependent confirmation of change in the interacting medium. Finally, the elastic force shows a strong correlation with the SANM signal, suggesting it as the main dissipation channel responsible for the mechanical coupling with sample.

\subsection{Mica}

Muscovite mica is a silicate crystal which present remarkable cleaving properties and an atomically flat surface. These properties make it suitable for adsorbed water layer experiments. Different groups have used SPM to analyze the adsorbed layer on mica at ambient conditions[117]-[120]. Despite being a commonly known hydrophilic surface, studies in muscovite mica have shown that the adsorbed water layer at ambient conditions has between 1 and 5 monomolecular layers[106], [118], [121], [122]. Apart from its hydrophilic properties, the nature of the water adsorption on mica remains a challenging subject. 


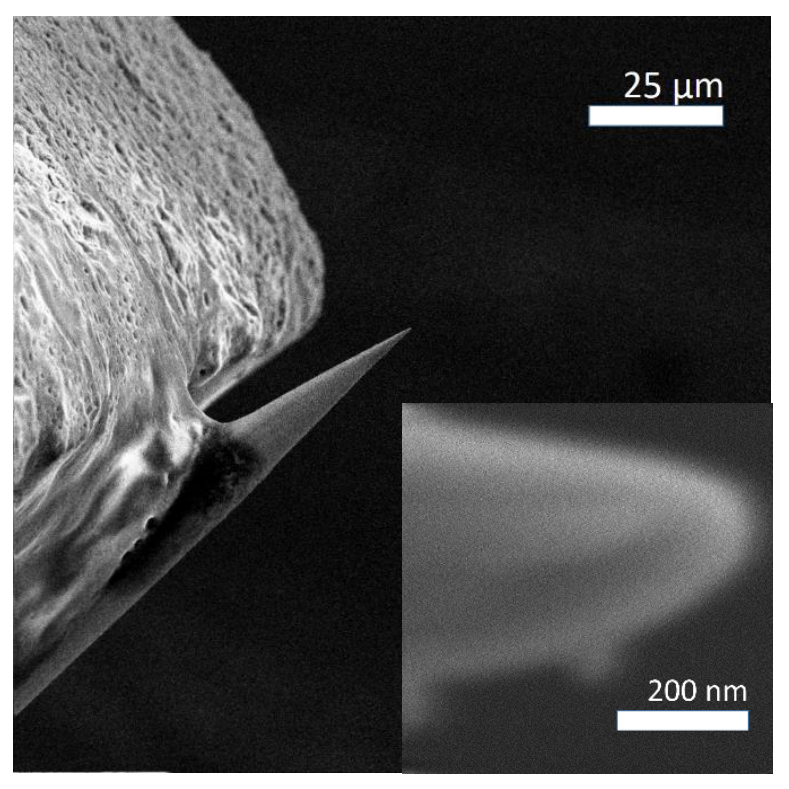

Figure 81. Probe used for the mica approach process.

\subsection{A Sample preparation}

A grade v1 mica disc from Ted Pella was cleaved with a sharp knife and placed on the surface of the SANM sensor using vacuum grease as a mechanical coupler. There were no electrical connections for this sample. After this point all procedures were similar to those followed for the HOPG sample. In this experiment the QTF is used as the control signal, since mica has the thinnest adsorbed layer of the three samples analyzed[106], [118], it requires the maximum sensitivity available in our system. The freshly mounted probe used in this process is shown in Figure 81. 


\subsection{B Initial approaches}

While still recording the SANM signal, the control signal used in this process is the QTF's. The parameters of the probe and the conditions are $Q=5893$, peak amplitude of $18 \mathrm{~nm}$, resonance frequency of $32021 \mathrm{~Hz}$, relative humidity $35 \pm 1 \%$ and a temperature of $27^{\circ}$ Celsius. The probe's parameters are: probe radius of approximately $80 \mathrm{~nm}$, a base radius of $5.7 \mu \mathrm{m}$ and a protruding length of $35 \mu \mathrm{m}$ from the edge of the QTF's tine. The driving force is calculated to be $80 \mathrm{nN}$, according to equation ( 12 ).

The first approach with a velocity of $5 \mathrm{~nm} / \mathrm{s}$ assures that the first contact does not reduce the oscillation amplitude in more than $30 \%$ and $400 \mathrm{mHz}$ in frequency shift.

One of the first controlled approaches is shown in Figure 82. The viscosity increases the forces involved in the retraction by about 5 times. This is consistent with adhesion measurements performed on mica and $\mathrm{SiO}_{2}[119]$ where mica showed a comparatively higher adhesion forces. There is also an oscillation pattern found in every initial curve, which will be discussed later. The interaction layer seems to be about $15 \AA$ before the frequency modulation losses its phase-lock. As explained in chapter 5, the phase between probe excitation and probe motion is maintained at $90^{\circ}$ by a PID loop. If the phase difference changes too fast (due to the change in force interaction between probe and layer (or sample) the PID cannot react fast enough and the signal stops tracking the phase. This sudden jump is observed right before the arbitrary zero in Figure 82. 


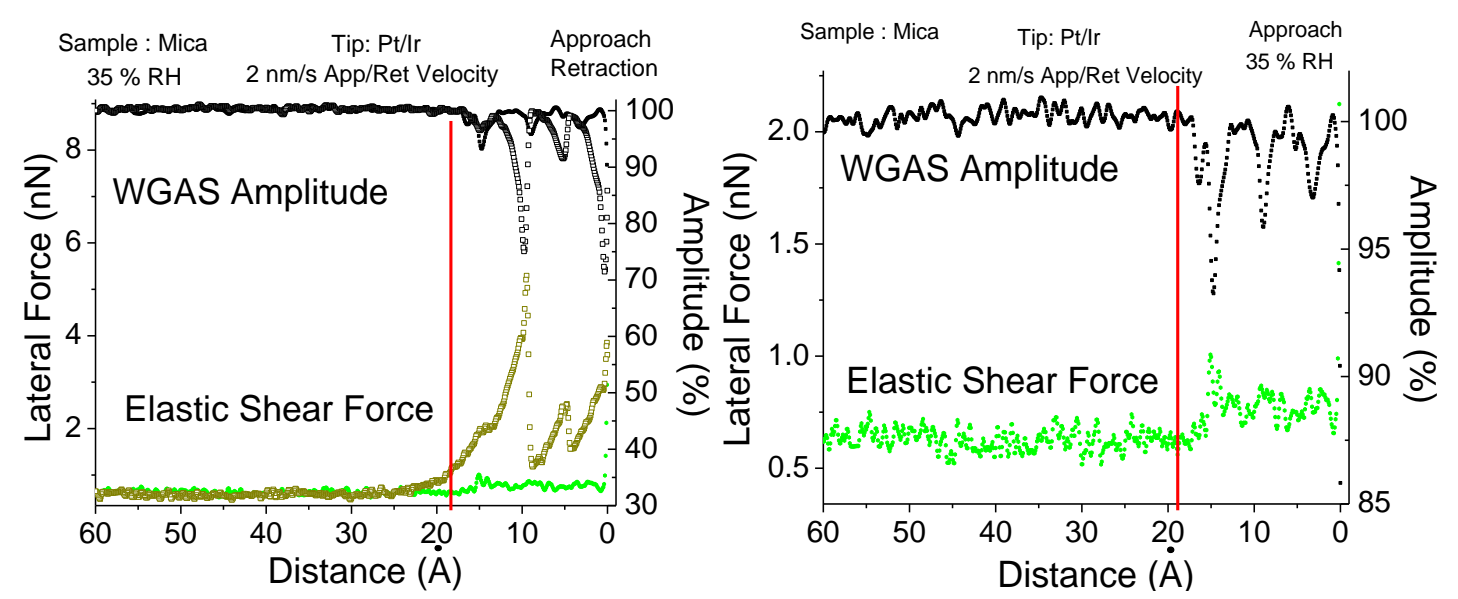

Figure 82. The first ARC on mica in air. Open markers are the retraction curves. The oscillating pattern repeats every $5 \AA$ approximately. Right before the zero both signals increase suddenly indicating the end of the frequency modulation regime.

As in the case of the HOPG sample, there is a region of constant elastic force right after the initial contact with the layer. The interaction region lasts for $15 \AA$ before the signal increases approximately 5 -fold, falling out of frequency modulation lock. Owing to its relatively thin adsorbed layer and the relatively large drift velocity (from $0.1 \mathrm{~nm} / \mathrm{s}$ to 1 $\mathrm{nm} / \mathrm{s}$ in extreme cases) the minimum approach velocity for a precise drift estimation was experimentally determined to be $2 \mathrm{~nm}$. As a consequence, the rate of force change when going from liquid-like layer interaction to a possible solid-like layer behavior is way above what the phase control PID can manage without substantially increasing the SNR of the system. Thus, increasing the phase PID's bandwidth would solve the control problem but the noise in the frequency detection would bury any measurable force levels. In this context, the mica sample tests the limits of our current system. 

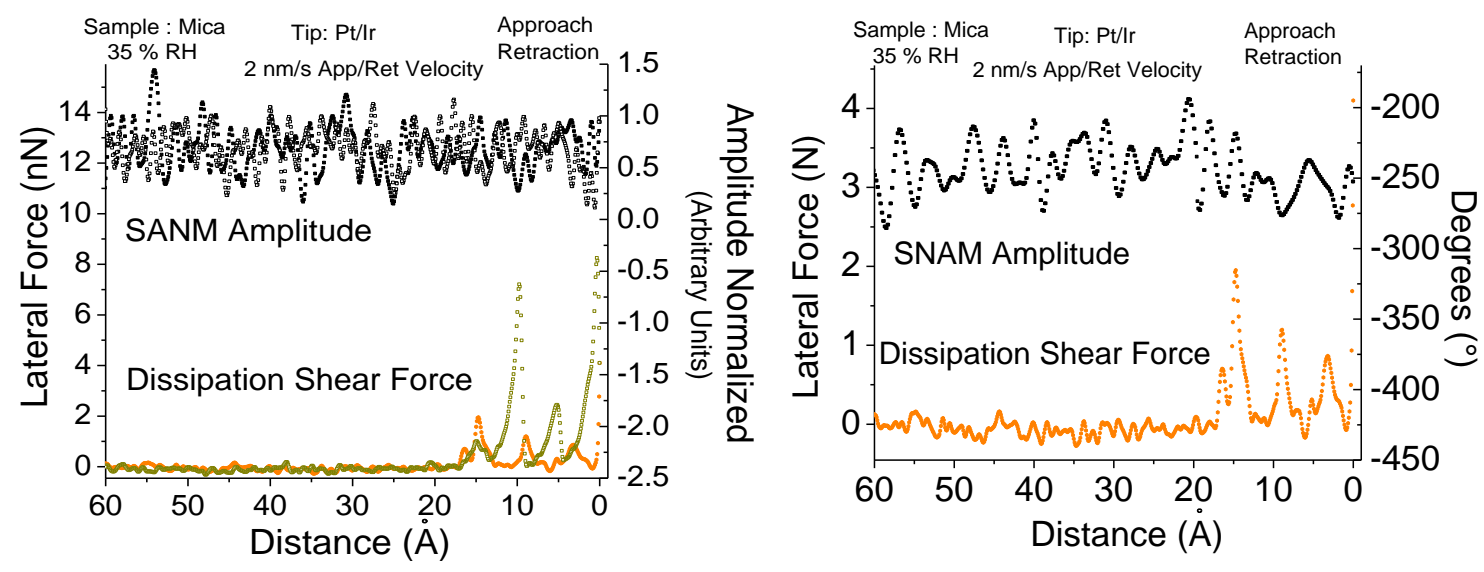

Figure 83. SANM amplitude and the dissipation force for the same curve in Figure 82. The periodic peaks are similar to the ones found in the elastic force and WGAS amplitude. The SANM presents no measurable signal at this point. The dissipation force signal shows the same periodic interaction peaks but the average dissipation force is still zero. The bottom sensor does not seem to record any oscillation either.

The retraction shows evidence of elastically detaching from the tip by the adsorbed layer, while at the same time dissipating energy. The three peaks are now puling the probe downwards, as can be elucidated from the direction of the oscillating peaks. The force the layer exert on the probe range from $3 \mathrm{nN}$ to $10 \mathrm{nN}$.

It has been observed that compressed liquid underneath the probing body becomes ordered in discrete layers[121], [123]. The periodicity of the oscillations is approximately $5 \AA$, which is equivalent to twice the length of a water molecule and previous studies have reported features with $5 \AA$ in height in mica surfaces at ambient conditions[124]. 
However, measurements with an improved drift compensation system are needed to conclusively determine the nature of the measured oscillations.

\subsection{Subsequent approaches}

Again, showing similarities with the HOPG experiment, the curves start to minimize their hysteresis after performing numerous approaches, as shown in Figure 84 . The maximum elastic force value during the approach is almost constant (around $2 \mathrm{nN}$ ). These approaches show that for a similar interaction force, the interaction length has increased from $18 \AA$ (Figure 82 ) to $6 \AA$ (Figure 84 ) in the approach and from $25 \AA$ to $39 \AA$ in the retraction.
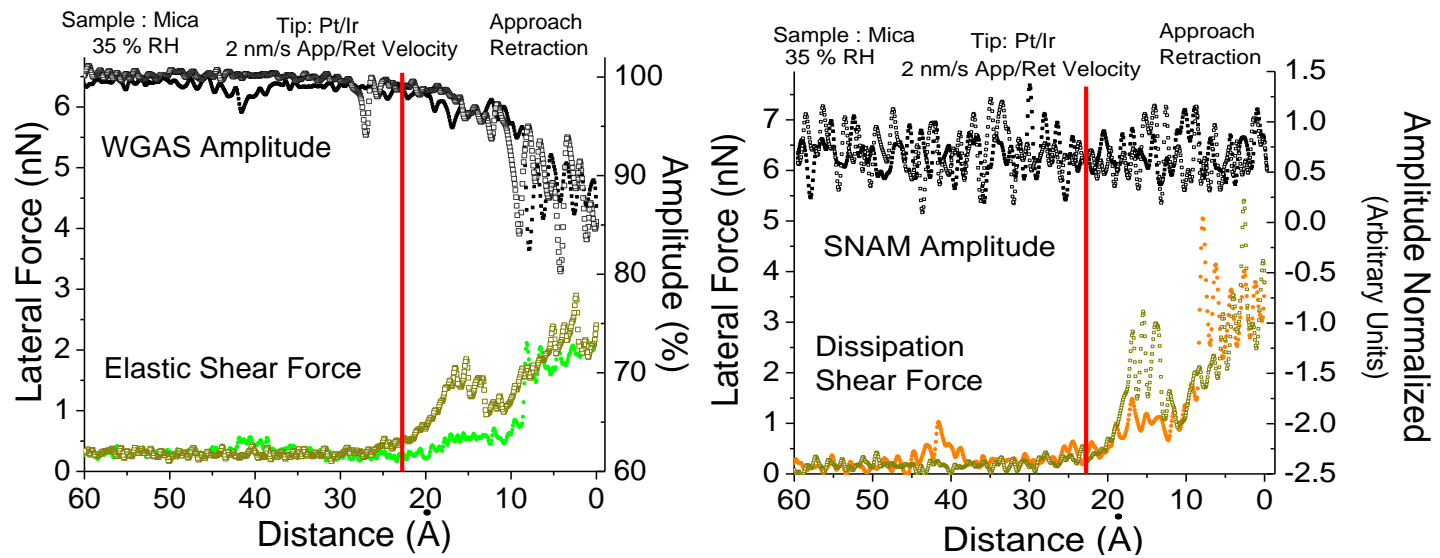

Figure 84. Curve showing reduced hysteresis. After several ARCs the curves the SANM Amplitude and dissipation force showing reduced hysteresis. 
As the probe keeps contacting the adsorbed layer, an increasing the amount of water gets accumulated near and around the interaction region. Capillarity induced water formation seems to increase the viscosity shown in the ARCs.

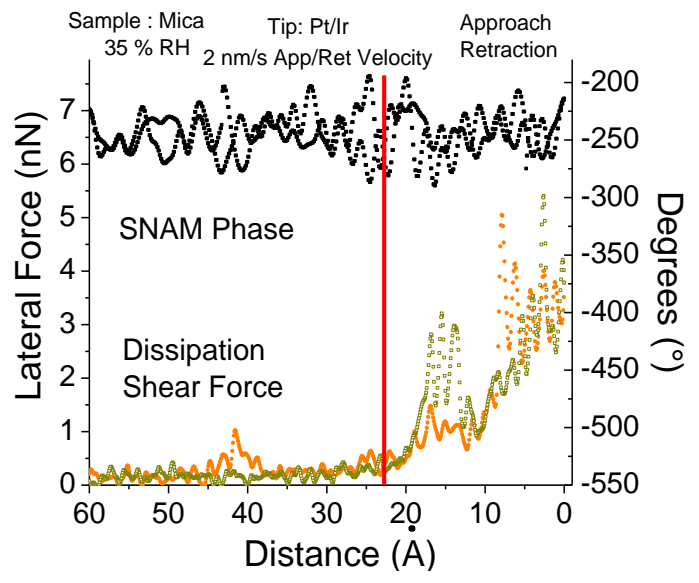

Figure 85. SANM Amplitude and dissipation force showing reduced hysteresis as interaction between probe and sample happens repeatedly.

After several curves the periodic peaks stop being the main feature and an average monotonic interaction appears. This is evidence of an increase of the adsorbed water layer thickness due to probe-induced condensation and/or regular condensation.

The layers closes to the surface are only able to be pushed aside when using a shear motion [125]. However, no SANM signal is detected. This could be due to the inability of the system to handle sharp force changes. There have been slight indications of the surge of the signal but are still below the noise level.

\subsection{Analysis of subsequent approaching and retracting curves (ARCs)}

The ARCs on mica represented a challenge and it tested the limits of the system. In its present developmental stage, the drift control is only effective when the drift 
velocity is at least 4 times smaller than the approach velocity and, as explained above the minimum practical approach velocity is $2 \mathrm{~nm} / \mathrm{s}$. As noted in the beginning of the section, the difficulty arises in the ability of the system to maintain the PI loop that controls the phase, thus achieving frequency modulation. Because of the inability to perform ARCs with lower velocities, the force change on the probe when encountering the surface or interacting with the solid-like behavior of the layer prevented a higher number of successful ARCs.

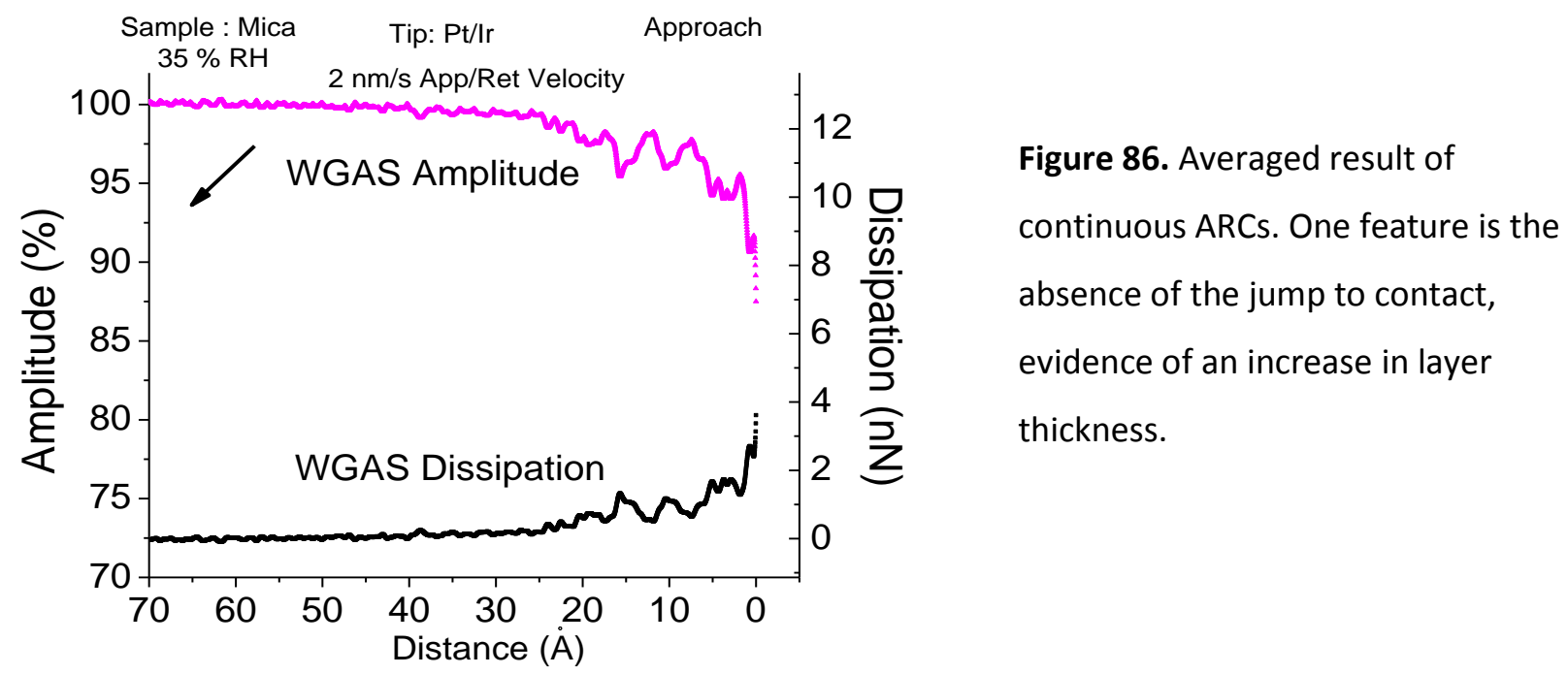

The averaged curves show the oscillation is still present. While not discarding a mechanical oscillation originating from mechanical sources (in the order of a few angstroms) the oscillations could be an indications of molecular layering of the confined fluid. 

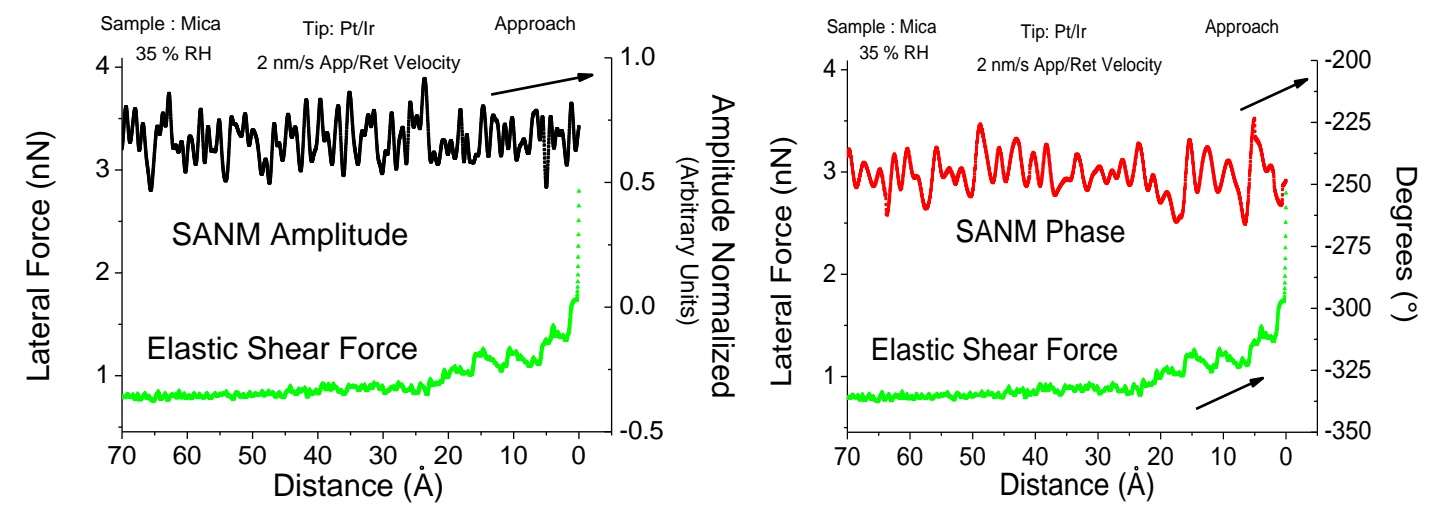

Figure 87. Elastic force compared with the SANM amplitude. No evidence of SANM signal, but the sudden jump to higher forces is noticeable at the end of the approach.

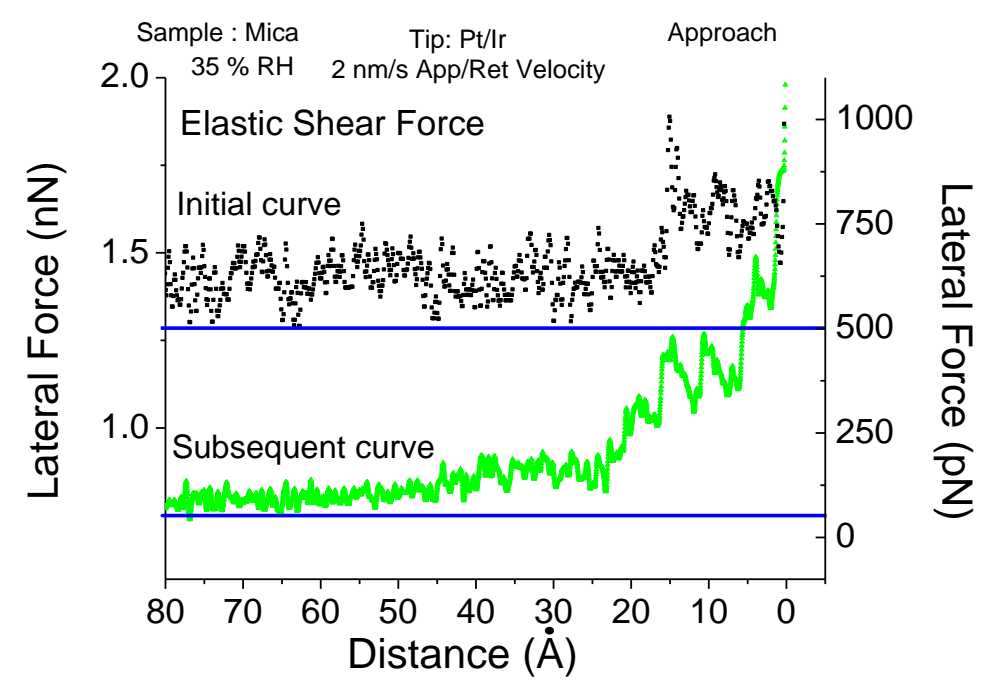

Figure 88. Comparison of length of interaction after several processes. This is believed to be caused by the change in the probe-surface contact area. 

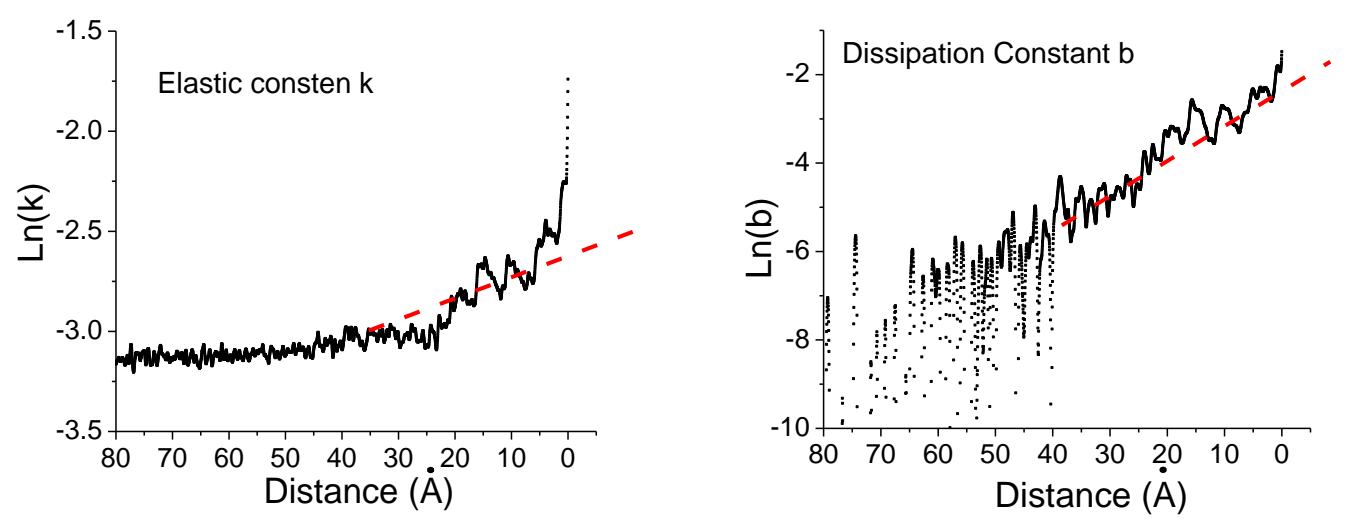

Figure 89 The logarithm WGAS elastic constant has a constant slope for approximately $15 \AA$ before dramatically increasing. The logarithm of the dissipation force showing a constant slope, evidence of viscous forces.

The later curves showed an increase of the interaction length (Figure 88), in accordance with the increase of the contact area due to repeated encounters with a solid like fluid (or the surface itself). The unique hydrophilic nature of mica may also explain why the interaction changes so rapidly distance, since the adhesion forces of the adsorbed layer have been found to be higher than in silicon oxide, another hydrophilic surface[119]. There is also strong evidence of the existence of ice-like water layer in the surface of mica in air[126], [127].

The exponential behavior is still present in both the dissipative and the elastic constants with the dissipation force extending until the arbitrary zero is reached. In this case, such a sharp increase in the interaction forces may originate in the layers' thickness. Smaller amounts of molecules could cause the transition from liquid-like to 
solid-like behavior to happen within a shorter region (and thus in shorter time) than on samples with thicker adsorbed layers. Due to the minimum limit in the approach velocity of our system $(2 \mathrm{~nm} / \mathrm{s})$, longer transition times are beyond reach.

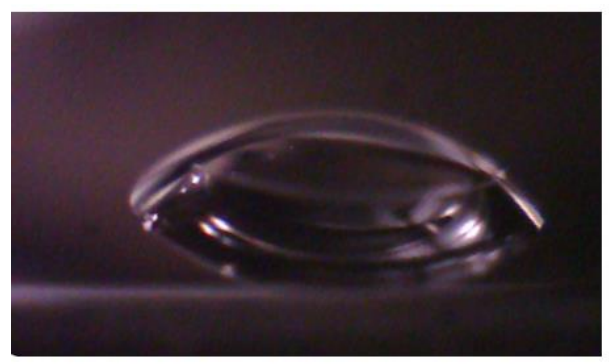

Drop of water on silicon

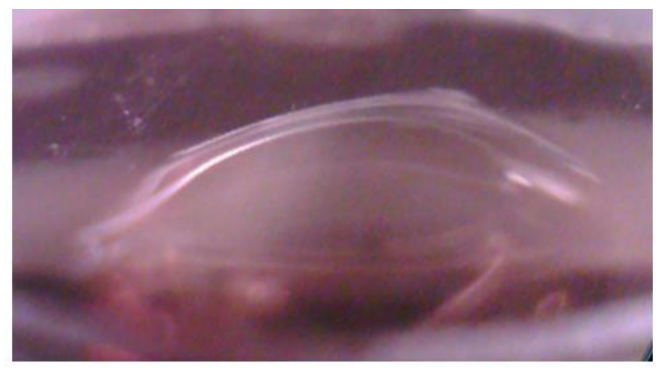

Drop of water on freshly cleaved mica

Figure 90. A volume of $1 \mathrm{ml}$ of de-ionized (DI) water placed on the silicon and cleaved mica used in the measurements. Both surfaces show hydrophilic behavior.

\subsection{E Section conclusions}

The ARCs on mica show a layer of approximately $20 \AA$ to $30 \AA$, in accordance with previously reported results[106]. The characteristic exponential behavior of the dissipation can be observed and a large force gradient after approximately $20 \AA$ into the interaction with the adsorbed layer occurs for the initial curves and $60 \AA$ for the subsequent curves. This is evidence of a transition caused by a sudden solid-like interaction suggesting an abrupt change of the viscoelastic properties. Further probing of the adsorbed layer requires improvement of the current implementation of the WGAS-SANM system. 


\subsection{Hydrogen terminated Silicon}

\subsection{A Sample preparation}

A $1 \mathrm{~cm} \mathrm{X} 1 \mathrm{~cm}$ square was cut from a $150 \mathrm{~cm}$ diameter silicon wafer. The sample was cleaned in a solution of $50 \%$ acetone (99\% purity) and $50 \%$ isopropanol (99\% purity) for 30 minutes in a sonicating bath. It was subsequently submerged in HF $49 \%$ (aqueous solution) for $30 \mathrm{~s}$ and cleaned with running de-ionized water (DI) water for another $30 \mathrm{~s}$. A stream of nitrogen gas was applied for $30 \mathrm{~s}$ to remove residual elements from its surface. Immediately afterwards, the silicon sample was placed on the SANM sensor using vacuum grease as a mechanical coupler, ready for measurements. The freshly mounted probe used in this process is shown in Figure 91.

\subsection{B Stable approaches}

While still recording the SANM signal, the QTF's signal is used to produce the control signal in this process. The parameters of the probe and the conditions are $\mathrm{Q}=2,526$, peak amplitude of $10 \mathrm{~nm}$, resonance frequency of $31,461 \mathrm{~Hz}$, relative humidity $43 \pm 1 \%$ and a temperature of $27^{\circ}$ Celsius. The probe's parameters are: probe radius of approximately $120 \mathrm{~nm}$, a base radius of 25 microns, and a protruding length of $80 \mu \mathrm{m}$ from the edge of the QTF's tine. Equation ( 12 ) sets the driving force at $103 \mathrm{nN}$. 


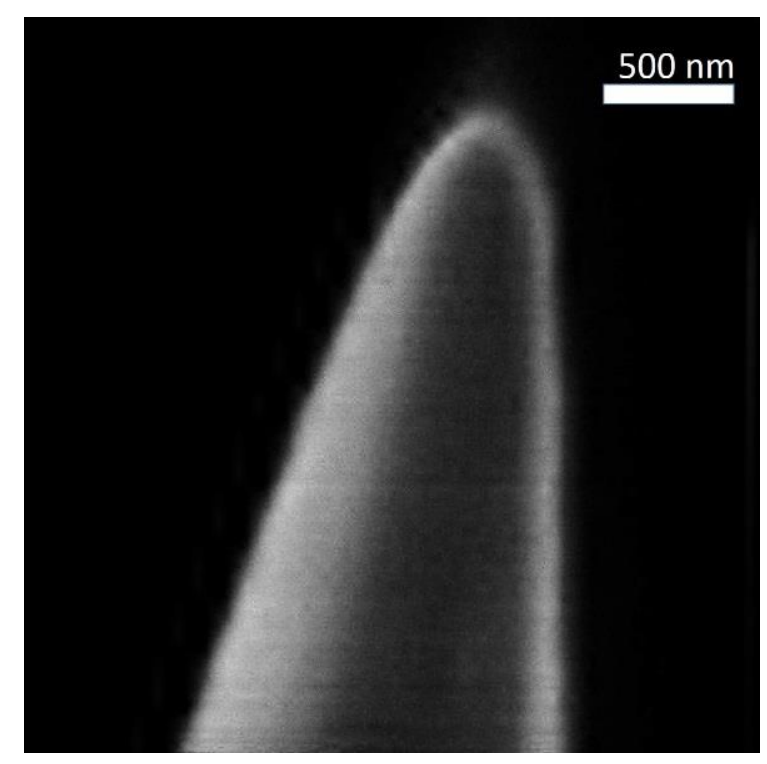

Figure 91. Probe used in the process on hydrogen terminated silicon.

From the analysis of previous surfaces (HOPG, mica) the SANM sensor is well correlated to the elastic force of the probe-layer interaction. This fact provides a method to determine, with increased certainty, whether the probe has reached a region of stronger mechanical coupling.

In the case of silicon, we found evidence of a thick layer on its surface. Figure 93 shows a first set of averaged curves where an interaction range extending approximately $1,100 \AA$ is observed. For the initial processes we did not obtain any significant SANM signals due to the length of the interaction layer. Thus we only present the curves that show contact with the layer adsorbed to the surface, as evidenced by the change in the SANM phase. The SANM normalized amplitude seems to increase in the last $1,100 \AA$. The SANM amplitude without normalization starts decreasing at the $400 \AA ̊$ mark and a slight increase at the $100 \AA$ mark. 

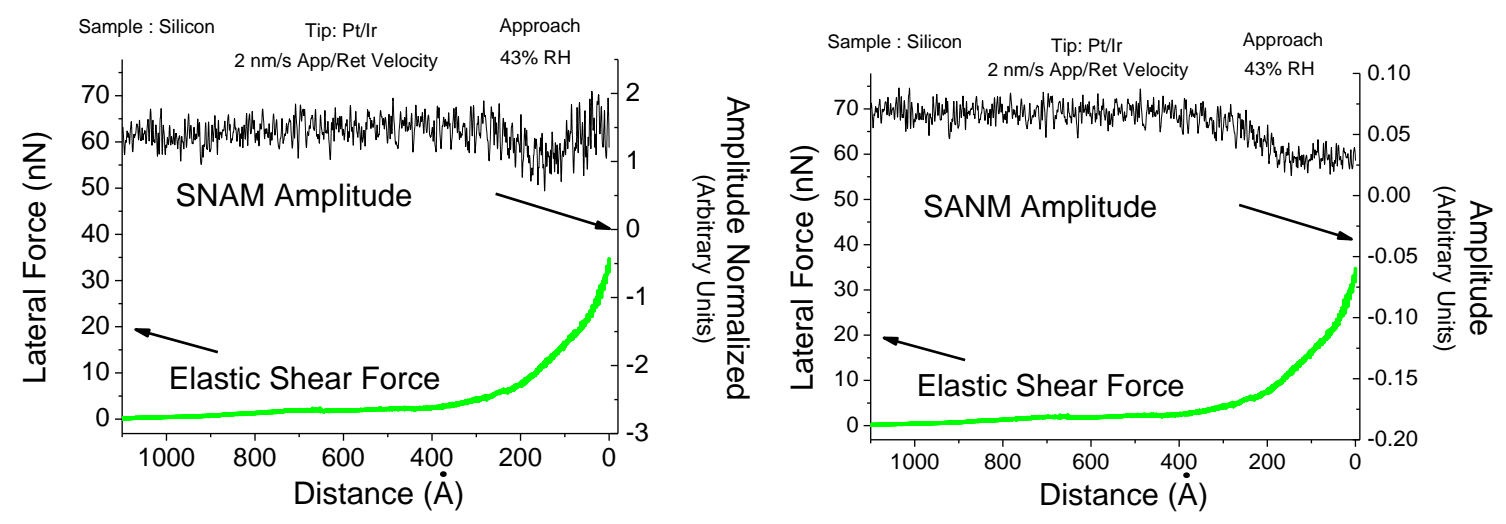

Figure 92. Comparison between normalized and unnnormalized SANM amplitude signal. The elastic shear force shows the characteristics exponential behavior indicating the viscous drag force of the layer.

However the normazliation of the SANM signal is justified by the SANM phase change observed, which indicates mechanical coupling between probe and sample (Figure 93).
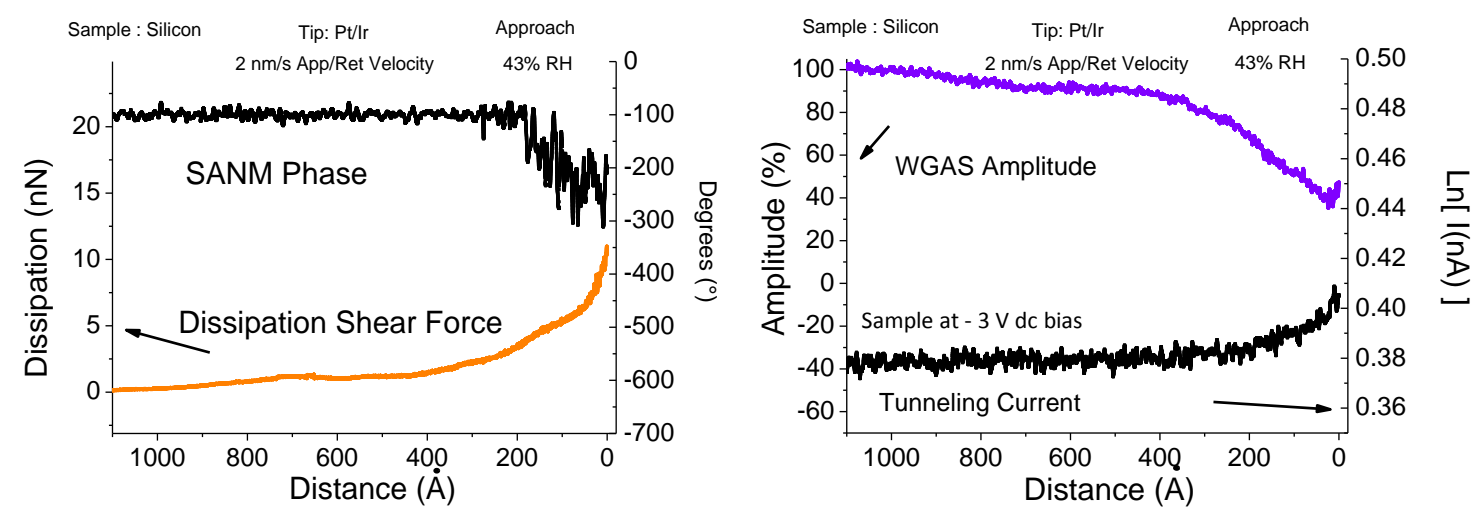

Figure 93. Left: The SANM phase shows a change which seems to indicate mechanical coupling with the with the surface. Right: The tunneling current starts at apporximately at the $350 \AA$ A mark. 
After the onset of the tunneling current both dissipation and elastic constants have a similar trend. Given that the silicon surface has a higher hardness rating than PtIr $10 \%$, a deformation of the probe is expected upon true contact with the sample. This differs from the approach curves on HOPG where during the transition curve, the elastic force had a negative slope, effectively measuring a decrease of the probe's elastic force, suggesting that the HOPG surface was elasticly complying under the probes's pressure, preventing it from detecting an increase in the elsatic constant[128], [129].

The tunneling current starts showing its exponential behavior at around $350 \AA$ from the arbitrary zero coinciding with a more stable behavior in the elastic and dissipation constants as evidenced in Figure 94.
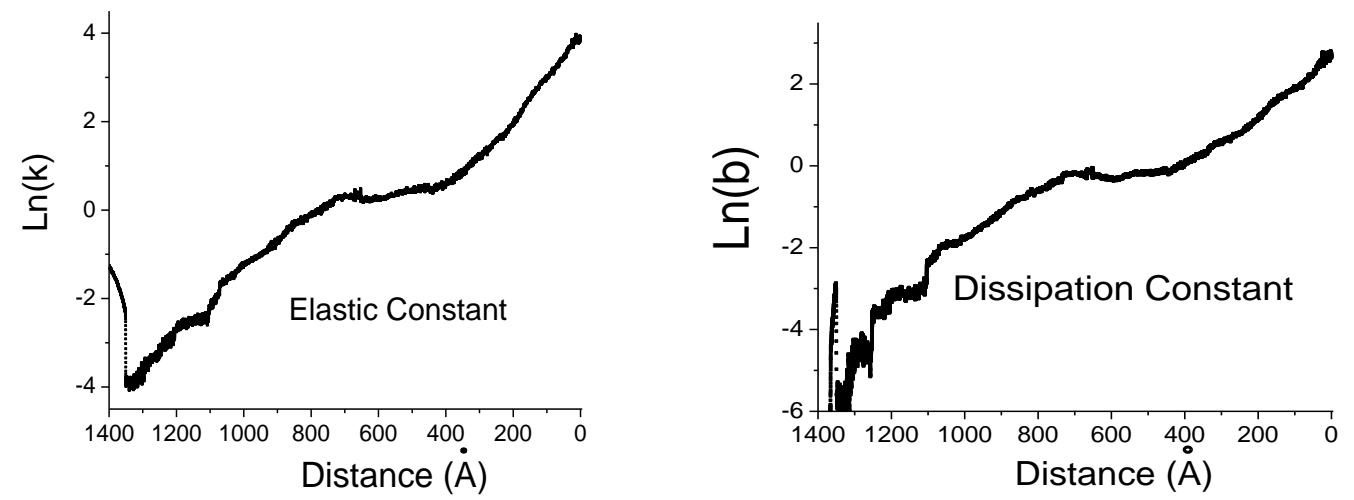

Figure 94. The natural logarithm of the dissipation and elastic constants in the first averaged set of approaches for silicon. The slope shows an approximately constant $b$ after the onset of the tunneling currentand (around the $350 \AA$ mark). Notice how both curves have an approximately constant slope, only to experience changes at around the $25 \AA$ A mark. 
In the case of silicon, the existence of silicon dihydrates[130], [131] after obtaining a hydrogen terminated surface by submerging the silicon sample in hydrofluoric acid (HF), may be the main component of the thick adsorbed layer found in the ARC results (Figure 92-93). A second set of averaged curves is shown in Figure 95-96. In similar fashion to the first set of averaged curves, the un-normalized SANM signal shows no evidence of mechanical coupling (Figure 95), but the phase change (Figure 96) indicates a mechanical coupling is indeed underway, making the normalized SANM amplitude a measure of the probe-sample interaction.

Figure 97 shows that the constant slope of the logarithm of the dissipation constant $b$ changes around $50 \AA$ from the arbitrary zero, while the elastic constant slope remains constant. This seems to indicate both an elastic bending and deformation of the probe in a solid-like interaction.
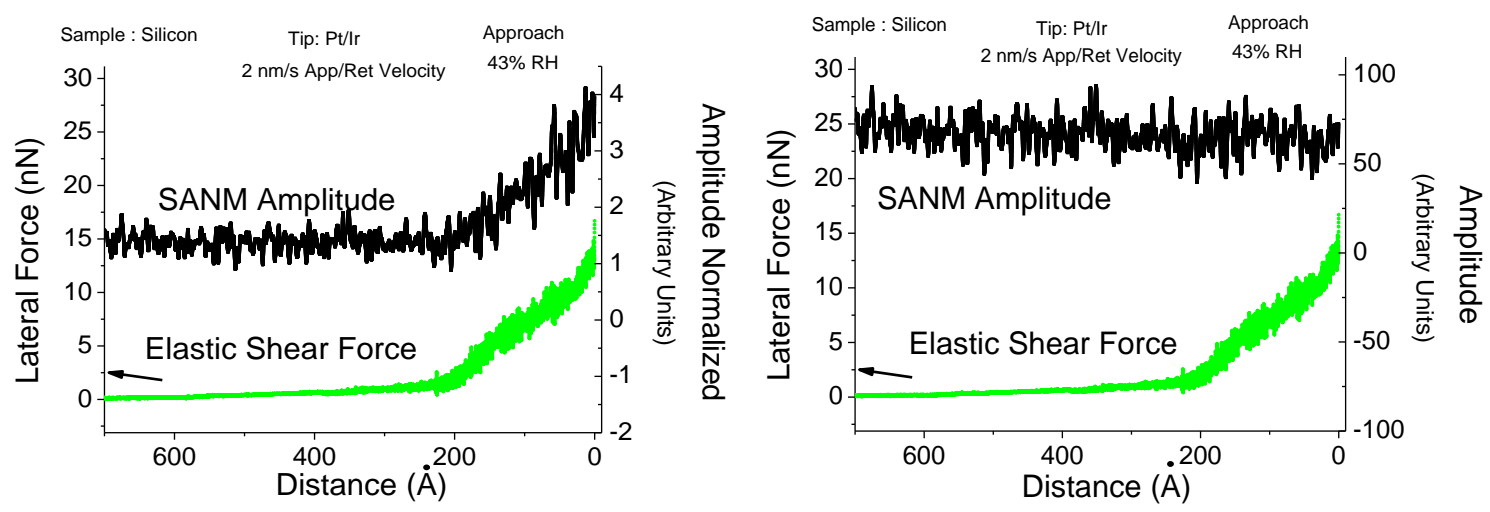

Figure 95. Comparison between normalized and unnnormalized SANM amplitude signal for the second averaged set. The SANM normalized amplitude (left) seems to increase in the last $200 \AA$ A. SANM amplitude without normalization (right) shows no evidence increase. 

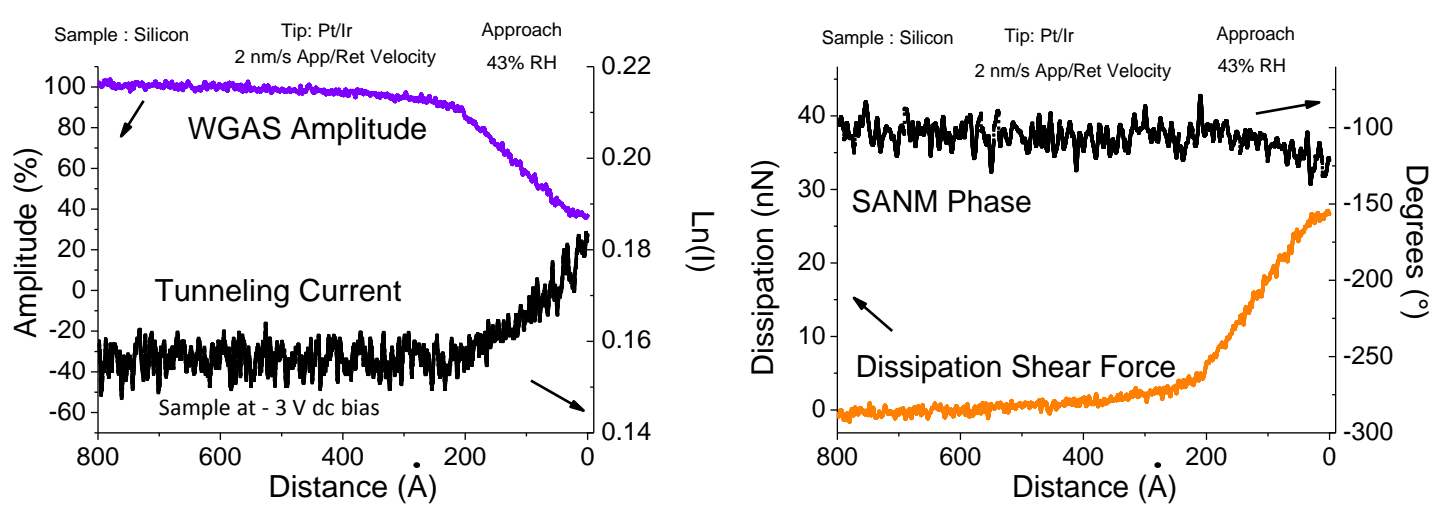

Figure 96. The WGAS amplitude shows an interaction region of at least $1000 \AA$. The last 50 $\AA ̊$ show a positive change in the slope, indicating a transition to a different medium. The tunneling current starts showing a linear behavior at around $250 \AA$ from the arbitrary zero. The SANM phase shows a small change which seems to indicate a soft contact with the surface.
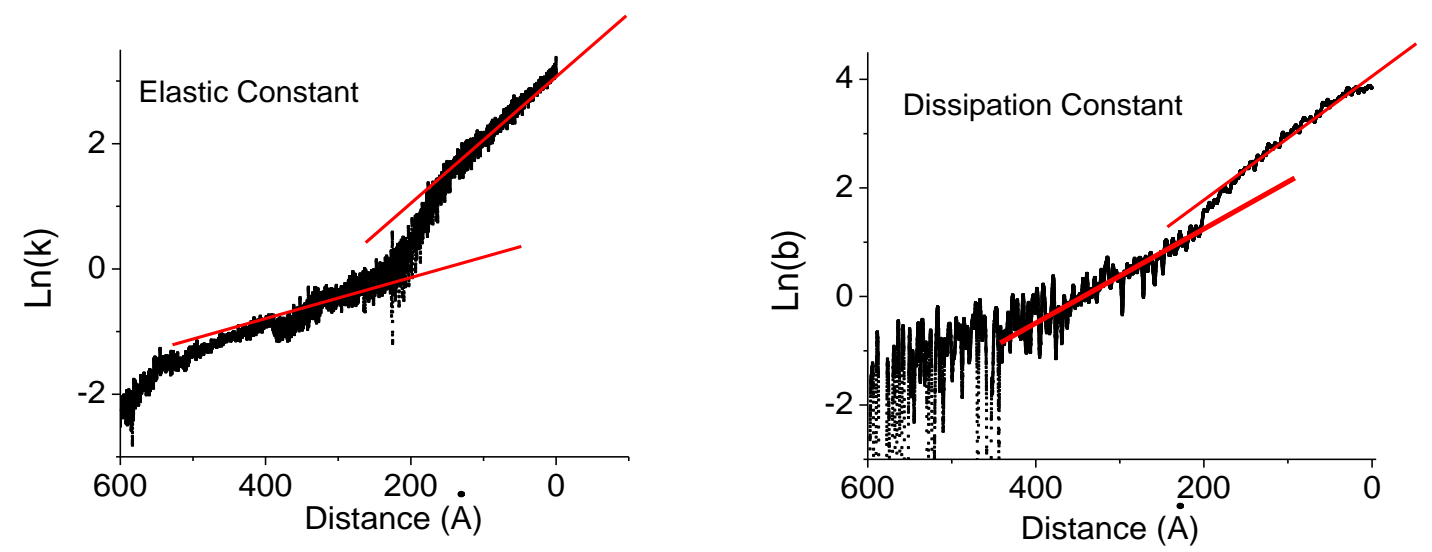

Figure 97. The natural logarithm of the elastic constant and dissipation constant in the second set of averaged approaches for silicon. The erratic behavior before the onset of the tunneling current ( $250 \AA$ mark) could be attributed to the thick layer found on the silicon surface.

Is this behavior that seems to indicate a contact with the solid-like confined layer (or silicon sample) since includes both a deformation (divergence of dissipation from 
exponential trend) and the elastic bending indicated by the exponential behavior of the elastic constant.

Modification of the cleaning protocol needs to be addressed. Clearly, the thick layer is the results of insufficient removal of byproducts of the cleaning procedure used. The semiconductor industry standard, RCA cleaning[132] needs to be included in the sample preparation protocol. Finally, Energy Dispersive X-Ray (EDX) analysis coupled with Raman and Fourier Transform Infrared (FTIR) Spectroscopy analysis would help determine the composition of the thick layer found on the sample's surface, as well as confirm or discard the existence of the silicon dihydrides[133].

\subsection{Section conclusions}

The ARCs on silicon show the exponential behavior of the dissipation force characteristic of the viscoelastic properties of the adsorbed surface layer. The tunneling current onset correlates very well with the increase of the force gradients, and the SANM phase signal. Notably, the SANM amplitude does not show much change in this region of higher force gradients. The SANM phase, on the contrary, shows a strong correlation with the transitions observed in dissipation and elastic forces providing confirmation, otherwise unavailable, of the onset of mechanical coupling between

probe and sample. Lastly, the normalized SANM amplitude, when present, show the same strong correlation with the elastic force observed in the HOPG experiments. 


\section{CHAPTER 7 \\ CONCLUSIONS}

We have shown the implementation of a quartz tuning fork (QTF) based scanning probe microscope (SPM) system with tunneling current, shear force and acoustic sensing capabilities, to perform approaching a retracting curves (ARC) on mesoscopic films at ambient conditions. The whispering gallery acoustic sensing (WGAS) and the shear acoustic near field microscopy (SANM) techniques presented strong evidence of their capabilities to measure the forces affecting the probe (WGAS) and the forces affecting the sample (SANM) when both are mechanically coupled through an adsorbed mesoscopic layer.

Results from ARCs performed on three different samples, with the system in frequency modulation mode enabling the decoupling of the interaction forces in elastic and dissipative, showed evidence of a correlation between elastic forces and the SANM amplitude. In HOPG the results point mainly towards an elastic dissipation channel for the probe-layer interaction. Strong correlations between the SANM phase, interaction forces and tunneling current measurements showed the relevance of the SANM phase measurements in the transition from liquid-like to solid like interactions experienced by the probe. A hydrogen terminated silicon surface presented evidence of mechanical coupling even in the absence of the SANM amplitude, with the help of the SANM phase. 
These results showcase the critical significance of SANM detection, as a measure of the relative phase between the probe and the adsorbed mesoscopic layer - when mechanically coupled to an oscillating probe - to study the dynamics of mesoscopic fluid under confinement. 


\subsection{Future Outlook}

Moving forward, the quantitative determination of the coupling between the probed surface and the SANM sensor would justify direct use of the model for the calculation of the phononic dissipation channel, proposed by our group[78]. Such a task would require a combination of numerical simulations using Finite Element Method (FEM) calculations and theoretical modeling to elucidate the coupling function for a given substrate.

In the experimental arena, scanning capabilities for in-situ imaging of the surface would enable pre and post imaging of the probed area, helping select an appropriate location for the ARCs. It also lends itself to 3D mapping of the probe-sample interactions, significantly incrementing the spectroscopy capabilities of the SANM-WGAS integrated system. In addition, while effective for sub-nanometer precision measurements and velocities above $2 \mathrm{~nm} / \mathrm{s}$, the thermal drift control implemented in this thesis would benefit from a SPM stage with a thermal drift compensation design that would render it suitable for inspection of samples with thin adsorbed layers such as mica.

Finally, the limitation placed by the use of the QTF excitation voltage as the bias voltage for the tunneling current measurements implies an inability to reduce the amount of tunneling current in the interaction region, minimizing heat effects. A technique to decouple these two voltage levels will diminish the likelihood of force contributions caused by high tunneling currents and its effects[113], [115]. 


\section{BIBLIOGRAPHY}

[1] S. Granick, "Motions and relaxations of confined liquids.," Science, vol. 253, no. 5026, pp. 1374-9, Sep. 1991.

[2] J. Klein and E. Kumacheva, "Confinement-induced phase transitions in simple liquids.," Science, vol. 269, no. 5225, pp. 816-9, Aug. 1995.

[3] D. Ortiz-Young, H.-C. Chiu, S. Kim, K. Voïtchovsky, and E. Riedo, "The interplay between apparent viscosity and wettability in nanoconfined water.," Nat. Commun., vol. 4, p. 2482, Jan. 2013.

[4] K. Jinesh and J. Frenken, "Capillary Condensation in Atomic Scale Friction: How Water Acts like a Glue," Phys. Rev. Lett., vol. 96, no. 16, p. 166103, Apr. 2006.

[5] M. Nosonovsky and B. Bhushan, "Phase behavior of capillary bridges: towards nanoscale water phase diagram.," Phys. Chem. Chem. Phys., vol. 10, no. 16, pp. 2137-44, Apr. 2008.

[6] K. Jinesh and J. Frenken, "Experimental Evidence for Ice Formation at Room Temperature," Phys. Rev. Lett., vol. 101, no. 3, p. 036101, Jul. 2008.

[7] E.-M. Choi, Y.-H. Yoon, S. Lee, and H. Kang, "Freezing Transition of Interfacial Water at Room Temperature under Electric Fields," Phys. Rev. Lett., vol. 95, no. 8, p. 085701, Aug. 2005.

[8] M. U. Hammer, T. H. Anderson, A. Chaimovich, M. S. Shell, and J. Israelachvili, "The search for the hydrophobic force law," Faraday Discuss., vol. 146, p. 299, 2010.

[9] K. Voïtchovsky, J. J. Kuna, S. A. Contera, E. Tosatti, and F. Stellacci, "Direct mapping of the solid-liquid adhesion energy with subnanometre resolution.," Nat. Nanotechnol., vol. 5, no. 6, pp. 401-5, Jun. 2010.

[10] E. Chiavazzo, M. Fasano, P. Asinari, and P. Decuzzi, "Scaling behaviour for the water transport in nanoconfined geometries.," Nat. Commun., vol. 5, p. 4565, Jan. 2014.

[11] C. Duan and A. Majumdar, "Anomalous ion transport in 2-nm hydrophilic nanochannels.," Nat. Nanotechnol., vol. 5, no. 12, pp. 848-52, Dec. 2010. 
[12] M. Ahmad, W. Gu, T. Geyer, and V. Helms, "Adhesive water networks facilitate binding of protein interfaces.," Nat. Commun., vol. 2, p. 261, Jan. 2011.

[13] W. Lv and R. Wu, "The interfacial-organized monolayer water film (MWF) induced 'two-step' aggregation of nanographene: both in stacking and sliding assembly pathways.," Nanoscale, vol. 5, no. 7, pp. 2765-75, Apr. 2013.

[14] H. Huang, Z. Song, N. Wei, L. Shi, Y. Mao, Y. Ying, L. Sun, Z. Xu, and X. Peng, "Ultrafast viscous water flow through nanostrand-channelled graphene oxide membranes.," Nat. Commun., vol. 4, p. 2979, Jan. 2013.

[15] A. Gil, J. Colchero, M. Luna, J. Gomez-Herrero, and A. Baro, "Adsorption of water on solid surfaces studied by scanning force microscopy," Langmuir, no. 11, pp. 5086-5092, 2000.

[16] E. Riedo, F. Lévy, and H. Brune, "Kinetics of Capillary Condensation in Nanoscopic Sliding Friction," Phys. Rev. Lett., vol. 88, no. 18, p. 185505, Apr. 2002.

[17] R. Szoszkiewicz and E. Riedo, "Nucleation Time of Nanoscale Water Bridges," Phys. Rev. Lett., vol. 95, no. 13, p. 135502, Sep. 2005.

[18] K. Lum, D. Chandler, and J. D. Weeks, "Hydrophobicity at Small and Large Length Scales," J. Phys. Chem. B, vol. 103, no. 22, pp. 4570-4577, Jun. 1999.

[19] J. Israelachvili and $\mathrm{H}$. Wennerström, "Role of hydration and water structure in biological and colloidal interactions.," Nature, vol. 379, no. 6562, pp. 219-25, Jan. 1996.

[20] B. N. J. Persson, Sliding Friction. Springer, 2000.

[21] S. H. Kim, D. B. Asay, and M. T. Dugger, "Nanotribology and MEMS," Nano Today, vol. 2, no. 5, pp. 22-29, Oct. 2007.

[22] H. M. Jaeger and S. R. Nagel, "Physics of the granular state.," Science, vol. 255, no. 5051, pp. 1523-31, Mar. 1992.

[23] T. M. Raschke, J. Tsai, and M. Levitt, "Quantification of the hydrophobic interaction by simulations of the aggregation of small hydrophobic solutes in water.," Proc. Natl. Acad. Sci. U. S. A., vol. 98, no. 11, pp. 5965-9, May 2001.

[24] K. B. Bikramjit Basu, Advanced Structural Ceramics. Wiley, 2011, p. 512. 
[25] M. Venturoli, M. Maddalenasperotto, M. Kranenburg, and B. Smit, "Mesoscopic models of biological membranes," Phys. Rep., vol. 437, no. 1-2, pp. 1-54, Dec. 2006.

[26] M. Cieplak, E. D. Smith, and M. O. Robbins, "Molecular origins of friction: the force on adsorbed layers.," Science, vol. 265, no. 5176, pp. 1209-12, Aug. 1994.

[27] U. Landman, R. N. Barnett, H. P. Cheng, C. L. Cleveland, and W. D. Luedtke, "Simulations of Materials: Clusters and Interfacial Junctions," in Computations for the Nanoscale, P. E. J. and A. J. F. Bloohl, Ed. Kluwer Dordrecht, 1993, pp. 75-113.

[28] J. Gao, W. D. Luedtke, and U. Landman, "Structure and solvation forces in confined films: Linear and branched alkanes," J. Chem. Phys., vol. 106, no. 10, p. 4309, 1997.

[29] M. Robbins and J. Krim, "Energy dissipation in interfacial friction," MRS Bull., no. JUNE, pp. 23-26, 1998.

[30] J. Krim, "Surface science and the atomic-scale origins of friction: what once was old is new again," Surf. Sci., vol. 500, no. 1-3, pp. 741-758, Mar. 2002.

[31] J. Israelachvili, Intermolecular and Surface Forces. New York: Academic Press, 1992.

[32] J. Lyklema, H. P. van Leeuwen, and M. Minor, "DLVO-theory, a dynamic reinterpretation," Adv. Colloid Interface Sci., vol. 83, no. 1-3, pp. 33-69, Dec. 1999.

[33] B. Bhushan, "Nanotribology and nanomechanics," vol. 259, pp. 1507-1531, 2005.

[34] H. K. Christenson and P. M. Claesson, "Direct measurements of the force between hydrophobic surfaces in water," Adv. Colloid Interface Sci., vol. 91, no. 3, pp. 391436, Jul. 2001.

[35] T. Ederth and B. Liedberg, "Influence of wetting properties on the long-range 'hydrophobic' interaction between self-assembled alkylthiolate monolayers," Langmuir, no. 16, pp. 2177-2184, 2000.

[36] S. O. and B. W. N. V. V. Yaminsky, "Long-range hydrophobic forces due to capillary bridging," in Handbook of Surfaces and Interfaces of Materials, H. S. Nalwa, Ed. Academic Press.

[37] E. M. Lifshitz, “No Title," Sov. Phys. JETP (Engl. Transl.), vol. 2, no. 73, 1956. 
[38] I. E. Dzyaloshinskii, E. M. Lifshitz, and L. P. Pitaevskii, "The general theory of van der Waals forces," Adv. Phys., vol. 10, no. 38, pp. 165-209, 1961.

[39] B. W. Ninham and P. Lo Nostro, Molecular Forces and Self Assembly. Cambridge: Cambridge University Press, 2010.

[40] J. N. Israelachvili, P. M. McGuiggan, and A. M. Homola, "Dynamic properties of molecularly thin liquid films.," Science, vol. 240, no. 4849, pp. 189-91, Apr. 1988.

[41] J. N. Israelachvili, "Thin film studies using multiple-beam interferometry," J. Colloid Interface Sci., vol. 44, no. 2, pp. 259-272, 1973.

[42] M. Heuberger, "The extended surface forces apparatus. Part I. Fast spectral correlation interferometry," Rev. Sci. Instrum., vol. 72, no. 3, p. 1700, 2001.

[43] J. Israelachvili and R. Pashley, "The hydrophobic interaction is long range, decaying exponentially with distance," Nature, vol. 300, no. 5890, pp. 341-342, Nov. 1982.

[44] J. Van Alsten and S. Granick, "Molecular Tribometry of Ultrathin Liquid Films," Phys. Rev. Lett., vol. 61, no. 22, pp. 2570-2573, Nov. 1988.

[45] J. Peachey, J. Van Alsten, and S. Granick, "Design of an apparatus to measure the shear response of ultrathin liquid films," Rev. Sci. Instrum., vol. 62, no. 2, p. 463, 1991.

[46] D. Tabor and R. H. Winterton, "Surface forces: direct measurement of normal and retarded van der Waals forces.," Nature, vol. 219, no. 5159, pp. 1120-1, Sep. 1968.

[47] J. Israelachvili, "Techniques for Direct Measurements of Forces Between Surfaces in Liquids at the Atomic Scale," Chemtracts - Anal. Phys. Chem., vol. 1, pp. 1-12, 1989.

[48] J. Klein, D. Perahia, and S. Warburg, "Forces between polymer-bearing surfaces undergoing shear," Nature, vol. 352, no. 6331, pp. 143-145, Jul. 1991.

[49] J. Krim, D. Solina, and R. Chiarello, "Nanotribology of a Kr monolayer: A quartzcrystal microbalance study of atomic-scale friction," Phys. Rev. Lett., vol. 66, no. 2, pp. 181-185, 1991. 
[50] J. Krim, "Sliding friction measurements of physisorbed monolayers: A comparison of solid and liquid films," J. Vac. Sci. Technol. B Microelectron. Nanom. Struct., vol. 9, no. 2, p. 1343, Mar. 1991.

[51] G. He, M. Muser, and M. Robbins, "Adsorbed layers and the origin of static friction," Science, vol. 284, no. 5420, pp. 1650-2, Jun. 1999.

[52] M. C. Giocondi, D. Yamamoto, E. Lesniewska, P.-E. Milhiet, T. Ando, and C. Le Grimellec, "Surface topography of membrane domains.," Biochim. Biophys. Acta, vol. 1798, no. 4, pp. 703-18, Apr. 2010.

[53] G. M. M. and J. N. Glosli, "Fundamentals of Friction: Microscopic and macroscopic processes," in Fundamentals of Friction: Microscopic and macroscopic processes, I. L. S. and H. M. Pollack, Ed. Kluwer, Dordrecht, 1992, p. 405.

[54] C. M. Mate, "Force microscopy studies of the molecular origins of friction and lubrication," IBM J. Res. Dev., vol. 39, no. 6, pp. 617-627, Nov. 1995.

[55] R. Carpick, N. Agrait, D. Ogletree, and M. Salmeron, "Variation of the interfacial shear strength and adhesion of a nanometer-sized contact," Langmuir, vol. 7463, no. 15, pp. 3334-3340, 1996.

[56] J. E. Houston, C. M. Doelling, T. K. Vanderlick, Y. Hu, G. Scoles, I. Wenzl, and T. R. Lee, "Comparative Study of the Adhesion, Friction, and Mechanical Properties of CF 3 - and CH 3 -Terminated Alkanethiol Monolayers," no. 7, pp. 3926-3932, 2005.

[57] S. An, M. Hong, J. Kim, S. Kwon, K. Lee, M. Lee, and W. Jhe, "Quartz tuning forkbased frequency modulation atomic force spectroscopy and microscopy with all digital phase-locked loop.," Rev. Sci. Instrum., vol. 83, no. 11, p. 113705, Nov. 2012.

[58] B. Kim, S. Kwon, H. Mun, S. An, and W. Jhe, "Energy dissipation of nanoconfined hydration layer: long-range hydration on the hydrophilic solid surface.," Sci. Rep., vol. 4, p. 6499, Jan. 2014.

[59] A. H. La Rosa, X. Cui, J. McCollum, N. Li, and R. Nordstrom, "The ultrasonic/shearforce microscope: Integrating ultrasonic sensing into a near-field scanning optical microscope," Rev. Sci. Instrum., vol. 76, no. 9, p. 093707, 2005.

[60] A. H. La Rosa, N. Li, R. Fernandez, X. Wang, R. Nordstrom, and S. K. Padigi, "Whispering-gallery acoustic sensing: Characterization of mesoscopic films and 
scanning probe microscopy applications," Rev. Sci. Instrum., vol. 82, no. 9, p. $093704,2011$.

[61] K. D. P. Günther, U. Ch. Fischer, "Scanning near-field acoustic microscopy," Appl. Phys. B, vol. Volume 48, no. Issue 1, pp. 89-92.

[62] G. Binnig, H. Rohrer, C. Gerber, and E. Weibel, "Surface studies by scanning tunneling microscopy," Phys. Rev. Lett., vol. 49, no. 1, pp. 57-61, 1982.

[63] G. Binnig and C. F. Quate, "Atomic Force Microscope," Phys. Rev. Lett., vol. 56, no. 9, pp. 930-933, Mar. 1986.

[64] U. Dürig, O. Züger, and a. Stalder, "Interaction force detection in scanning probe microscopy: Methods and applications," J. Appl. Phys., vol. 72, no. 5, p. 1778, 1992.

[65] K. Karrai and R. D. Grober, "Piezoelectric tip-sample distance control for near field optical microscopes," Appl. Phys. Lett., vol. 66, no. 14, p. 1842, 1995.

[66] A. G. T. Ruiter, K. O. van der Werf, J. A. Veerman, M. F. Garcia-Parajo, W. H. J. Rensen, and N. F. van Hulst, "Tuning fork shear-force feedback," Ultramicroscopy, vol. 71, no. 1-4, pp. 149-157, Mar. 1998.

[67] W. H. J. Rensen, N. F. van Hulst, A. G. T. Ruiter, and P. E. West, "Atomic steps with tuning-fork-based noncontact atomic force microscopy," Appl. Phys. Lett., vol. 75, no. 11, p. 1640, 1999.

[68] A. G. T. Ruiter, J. A. Veerman, K. O. van der Werf, and N. F. van Hulst, "Dynamic behavior of tuning fork shear-force feedback," Appl. Phys. Lett., vol. 71, no. 1, p. 28, 1997.

[69] W. A. Atia and C. C. Davis, "A phase-locked shear-force microscope for distance regulation in near-field optical microscopy," Appl. Phys. Lett., vol. 70, no. 4, p. 405, 1997.

[70] K. S. Van Dyke, "The Piezo-Electric Resonator and Its Equivalent Network," Proc. IRE, vol. 16, no. 6, pp. 742-764, Jun. 1928.

[71] D. W. Dye, "The piezo-electric quartz resonator and its equivalent electrical circuit," Proc. Phys. Soc. London, vol. 38, no. 1, pp. 399-458, Jan. 1925. 
[72] K. Karrai and R. D. Grober, "Tip-sample distance control for near field scanning optical microscopes," SPIE Proc., vol. 2535, pp. 69-81, Sep. 1995.

[73] J. Rychen, T. Ihn, P. Studerus, a. Herrmann, K. Ensslin, H. J. Hug, P. J. a. van Schendel, and H. J. Güntherodt, "Operation characteristics of piezoelectric quartz tuning forks in high magnetic fields at liquid helium temperatures," Rev. Sci. Instrum., vol. 71, no. 4, p. 1695, 2000.

[74] A. Castellanos-Gomez, N. Agraït, and G. Rubio-Bollinger, "Dynamics of quartz tuning fork force sensors used in scanning probe microscopy.," Nanotechnology, vol. 20, no. 21, p. 215502, May 2009.

[75] K. Karrai and I. Tiemann, "Interfacial shear force microscopy," Phys. Rev. B, vol. 62, no. 19, pp. 13174-13181, Nov. 2000.

[76] R. D. Grober, J. Acimovic, J. Schuck, D. Hessman, P. J. Kindlemann, J. Hespanha, a. S. Morse, K. Karrai, I. Tiemann, and S. Manus, "Fundamental limits to force detection using quartz tuning forks," Rev. Sci. Instrum., vol. 71, no. 7, p. 2776, 2000.

[77] A. E. Bate, "Note on the whispering gallery of St Paul's Cathedral, London," Proc. Phys. Soc., vol. 50, no. 2, pp. 293-297, Mar. 1938.

[78] X. Cui and A. La Rosa, "Investigation of the probe-sample interaction in the ultrasonic/shear-force microscope: The phononic friction mechanism," Appl. Phys. Lett., vol. 87, no. 23, p. 231907, 2005.

[79] R. Fernandez, X. Wang, and A. La Rosa, Acousto characterization of fluid-like mesoscopic films under shear. IEEE, 2011, pp. 903-906.

[80] A. H. La Rosa, B. I. Yakobson, and H. D. Hallen, "Optical imaging of carrier dynamics in silicon with subwavelength resolution," Appl. Phys. Lett., vol. 70, no. 13, p. 1656, 1997.

[81] U. Dürig, "Relations between interaction force and frequency shift in largeamplitude dynamic force microscopy," Appl. Phys. Lett., vol. 75, no. 3, p. 433, 1999.

[82] S. Ke, T. Uda, and K. Terakura, "Quantity measured in frequency-shift-mode atomic-force microscopy: An analysis with a numerical model," Phys. Rev. B, vol. 59, no. 20, pp. 13267-13272, May 1999. 
[83] F. Giessibl and H. Bielefeldt, "Physical interpretation of frequency-modulation atomic force microscopy," Phys. Rev. B, vol. 61, no. 15, pp. 9968-9971, Apr. 2000.

[84] J. Aimé, R. Boisgard, L. Nony, and G. Couturier, "Nonlinear Dynamic Behavior of an Oscillating Tip-Microlever System and Contrast at the Atomic Scale," Phys. Rev. Lett., vol. 82, no. 17, pp. 3388-3391, Apr. 1999.

[85] H. Hölscher, B. Gotsmann, W. Allers, U. Schwarz, H. Fuchs, and R. Wiesendanger, "Measurement of conservative and dissipative tip-sample interaction forces with a dynamic force microscope using the frequency modulation technique," Phys. Rev. B, vol. 64, no. 7, p. 075402, Jul. 2001.

[86] U. Dürig, "Interaction sensing in dynamic force microscopy," New J. Phys., vol. 2, no. 5, pp. 5.1-5.12, 2000.

[87] F. Giessibl, "Forces and frequency shifts in atomic-resolution dynamic-force microscopy," Phys. Rev. B, vol. 56, no. 24, pp. 16010-16015, Dec. 1997.

[88] M. J. Higgins, C. K. Riener, T. Uchihashi, J. E. Sader, R. McKendry, and S. P. Jarvis, "Frequency modulation atomic force microscopy: a dynamic measurement technique for biological systems," Nanotechnology, vol. 16, no. 3, pp. 585-589, Mar. 2005.

[89] F. J. Giessibl, "Advances in atomic force microscopy," Rev. Mod. Phys., vol. 75, no. July, 2003.

[90] X. Chen, M. C. Davies, C. J. Roberts, S. J. B. Tendler, P. M. Williams, and N. a. Burnham, "Optimizing phase imaging via dynamic force curves," Surf. Sci., vol. 460, no. 1-3, pp. 292-300, Jul. 2000.

[91] D. Müller and A. Engel, "The height of biomolecules measured with the atomic force microscope depends on electrostatic interactions," Biophys. J., vol. 73, no. September, 1997.

[92] G. Bar, Y. Thomann, R. Brandsch, and H. Cantow, "Factors Affecting the Height and Phase Images in Tapping Mode Atomic Force Microscopy. Study of PhaseSeparated Polymer Blends of Poly ( ethene- co -styrene ) and Poly ( 2 , 6-dimethyl1, 4-phenylene oxide )," Langmuir, vol. 7463, no. 3, pp. 3807-3812, 1997.

[93] T. E. Balmer, H. K. Christenson, N. D. Spencer, and M. Heuberger, "The effect of surface ions on water adsorption to mica.," Langmuir, vol. 24, no. 4, pp. 1566-9, Feb. 2008. 
[94] F. Moreno-Herrero, J. Colchero, and a M. Baró, "DNA height in scanning force microscopy.," Ultramicroscopy, vol. 96, no. 2, pp. 167-74, Aug. 2003.

[95] S. Santos, V. Barcons, H. K. Christenson, J. Font, and N. H. Thomson, "The intrinsic resolution limit in the atomic force microscope: implications for heights of nanoscale features.," PLoS One, vol. 6, no. 8, p. e23821, Jan. 2011.

[96] X. Ramus, Transimpedance Considerations for High-Speed Amplifiers, no. SBoa122. Texas Instruments, Application Report, SBOA-122, 2009.

[97] 1.6GHz, Low-Noise, FET-Input operational amplifier, no. December 2001. Texas Instruments, OPA657 Datasheet, 2008.

[98] T. Kugelstadt, Active Filter Design Techniques, SLOA-088 ed. Texas Instruments, Application Report, SLOA-088, 2008.

[99] S.-H. Park and G. Sposito, "Structure of Water Adsorbed on a Mica Surface," Phys. Rev. Lett., vol. 89, no. 8, p. 085501, Aug. 2002.

[100] M. Lee, J. Jahng, K. Kim, and W. Jhe, "Quantitative atomic force measurement with a quartz tuning fork," Appl. Phys. Lett., vol. 91, no. 2, p. 023117, 2007.

[101] J. Welker, F. de Faria Elsner, and F. J. Giessibl, "Application of the equipartition theorem to the thermal excitation of quartz tuning forks," Appl. Phys. Lett., vol. 99, no. 8, p. 084102, 2011.

[102] M. Song, J. Jang, and C. Lee, "Electron tunneling and electrochemical currents through interfacial water inside an STM junction," Bull. Korean Chem. Soc., vol. 23, no. 1, pp. 71-74, 2002.

[103] S. Park and C. F. Quate, "Tunneling microscopy of graphite in air," Appl. Phys. Lett., vol. 48, no. 2, p. 112, 1986.

[104] J. Hahn and H. Kang, "Vacancy and interstitial defects at graphite surfaces: Scanning tunneling microscopic study of the structure, electronic property, and yield for ion-induced defect creation," Phys. Rev. B, vol. 60, no. 8, pp. 6007-6017, Aug. 1999.

[105] J. Freund, "How dry are dried samples? Water adsorption measured by STM," Microsc. Res. Tech., vol. 44, pp. 327-338, 1999. 
[106] A. Opitz, M. Scherge, S. I.-U. Ahmed, and J. A. Schaefer, "A comparative investigation of thickness measurements of ultra-thin water films by scanning probe techniques," J. Appl. Phys., vol. 101, no. 6, p. 064310, 2007.

[107] L. Olesen, M. Brandbyge, M. Sorensen, K. Jacobsen, E. Lægsgaard, I. Stensgaard, and F. Besenbacher, "Apparent barrier height in scanning tunneling microscopy revisited.," Phys. Rev. Lett., vol. 76, no. 9, pp. 1485-1488, Feb. 1996.

[108] B. Bhikkaji and M. Ratnam, "High-performance control of piezoelectric tube scanners," IEEE Trans. Control Syst. Technol., vol. 15, no. 5, pp. 853-866, 2007.

[109] Noliac, "Piezoelectric Materials Characteristics," 2011. [Online]. Available: http://www.noliac.com/Material_characteristics_-143.aspx.

[110] J. Sader and S. Jarvis, "Coupling of conservative and dissipative forces in frequency-modulation atomic force microscopy," Phys. Rev. B, vol. 74, no. 19, p. 195424, Nov. 2006.

[111] J. Hahn, Y. Hong, and H. Kang, "Electron tunneling across an interfacial water layer inside an STM junction: tunneling distance, barrier height and water polarization effect," Appl. Phys. A, vol. 472, pp. 467-472, 1998.

[112] K. Yamasue and Y. Cho, "Simultaneous measurement of tunneling current and atomic dipole moment on $\mathrm{Si}(111)-(7 \times 7)$ surface by noncontact scanning nonlinear dielectric microscopy," J. Appl. Phys., vol. 113, no. 1, p. 014307, 2013.

[113] S. J. Stranick and P. S. Weiss, "Alternating Current Scanning Tunneling Microscopy and Nonlinear Spectroscopy," J. Phys. Chem., vol. 98, no. 7, pp. 1762-1764, Feb. 1994.

[114] G. Kochanski, "Nonlinear alternating-current tunneling microscopy," Phys. Rev. Lett., vol. 62, no. 19, pp. 2285-2288, 1989.

[115] R. Nishitani, F. Begum, and H. Iwasaki, "Alternating Current of Scanning Tunneling Microscope for Organic Molecules Adsorbed on Metal in Terms of Equivalent Circuit of Scanning Tunneling Microscope," Jpn. J. Appl. Phys., vol. 45, no. 3B, pp. 1962-1965, Mar. 2006.

[116] Z. Majzik, M. Setvín, A. Bettac, A. Feltz, V. Cháb, and P. Jelínek, "Simultaneous current, force and dissipation measurements on the Si(111) $7 \times 7$ surface with an optimized qPlus AFM/STM technique.," Beilstein J. Nanotechnol., vol. 3, no. 111, pp. 249-59, Jan. 2012. 
[117] A. Gil, J. Colchero, M. Luna, J. Gómez-Herrero, and A. M. Baró, "Adsorption of Water on Solid Surfaces Studied by Scanning Force Microscopy," Langmuir, vol. 16, no. 11, pp. 5086-5092, May 2000.

[118] G. E. Ewing, "Thin Film Water on Muscovite Mica," J. Phys. Chem. B, vol. 105, no. 23, pp. 5434-5439, Jun. 2001.

[119] F. L. Leite, E. C. Ziemath, O. N. Oliveira Jr., and P. S. P. Herrmann, "Adhesion Forces for Mica and Silicon Oxide Surfaces Studied by Atomic Force Spectroscopy (AFS)," Microsc. Microanal., vol. 11, no. S03, pp. 130-133, Dec. 2005.

[120] T. Fukuma, "Water distribution at solid/liquid interfaces visualized by frequency modulation atomic force microscopy," Sci. Technol. Adv. Mater., vol. 11, no. 3, p. 033003, Jun. 2010.

[121] M. Antognozzi, a. D. L. Humphris, and M. J. Miles, "Observation of molecular layering in a confined water film and study of the layers viscoelastic properties," Appl. Phys. Lett., vol. 78, no. 3, p. 300, 2001.

[122] S. Jeffery, P. Hoffmann, J. Pethica, C. Ramanujan, H. Özer, and A. Oral, "Direct measurement of molecular stiffness and damping in confined water layers," Phys. Rev. B, vol. 70, no. 5, p. 054114, Aug. 2004.

[123] D. Beaglehole and B. W. Ninham, "Molecular Layering in a Liquid Adsorbed Film at Room Temperature," no. 22, pp. 1843-1845, 1991.

[124] J. Hu, X. -d. Xiao, D. F. Ogletree, and M. Salmeron, "Atomic scale friction and wear of mica," Surf. Sci., vol. 327, no. 3, pp. 358-370, Apr. 1995.

[125] M. L. Gee, P. M. McGuiggan, J. N. Israelachvili, and A. M. Homola, "Liquid to solidlike transitions of molecularly thin films under shear," J. Chem. Phys., vol. 93, no. 3, p. 1895, 1990.

[126] J. Hu, X. D. Xiao, D. F. Ogletree, and M. Salmeron, "Imaging the condensation and evaporation of molecularly thin films of water with nanometer resolution.," Science, vol. 268, no. 5208, pp. 267-9, Apr. 1995.

[127] K. Xu, P. Cao, and J. R. Heath, "Graphene visualizes the first water adlayers on mica at ambient conditions.," Science, vol. 329, no. 5996, pp. 1188-91, Sep. 2010.

[128] S. An, J. Kim, K. Lee, B. Kim, M. Lee, and W. Jhe, "Mechanical properties of the nanoscale molecular cluster of water meniscus by high-precision frequency 
modulation atomic force spectroscopy," Appl. Phys. Lett., vol. 101, no. 5, p. 053114, 2012.

[129] Z. Deng, A. Smolyanitsky, Q. Li, X.-Q. Feng, and R. J. Cannara, "Adhesiondependent negative friction coefficient on chemically modified graphite at the nanoscale.," Nat. Mater., vol. 11, no. 12, pp. 1032-7, Dec. 2012.

[130] P. Gupta, V. Colvin, and S. George, "Hydrogen desorption kinetics from monohydride and dihydride species on silicon surfaces," Phys. Rev. B, pp. 82348243, 1988.

[131] K. Arima, K. Endo, T. Kataoka, Y. Oshikane, H. Inoue, and Y. Mori, "Scanning tunneling microscopy study of hydrogen-terminated Si(001) surfaces after wet cleaning," Surf. Sci., vol. 446, no. 1-2, pp. 128-136, Feb. 2000.

[132] W. Kern, "The Evolution of Silicon Wafer Cleaning Technology," J. Electrochem. Soc., vol. 137, no. 6, p. 1887, 1990.

[133] C.-H. Chung, "In situ infrared spectroscopic study on the role of surface hydrides and fluorides in the silicon chemical vapor deposition process," J. Vac. Sci. Technol. A Vacuum, Surfaces, Film., vol. 13, no. 6, p. 2698, Nov. 1995.

[134] J. Rychen, T. Ihn, P. Studerus, A. Herrmann, K. Ensslin, H. J. Hug, and H. J. Guntherodt, "Force-distance studies with piezoelectric tuning forks below $4.2 \mathrm{~K}$," Appl. Surf. Sci., vol. 157, pp. 290-294, 2000. 


\section{APPENDIX A}

\section{Calculation of the elastic and dissipation interaction forces}

Following the derivation of the equations for the elastic and dissipation forces, we combine equations ( 4 ) through ( 7 ),

$$
m\left(i \omega_{0}\right)^{2} x_{0} e^{-i \frac{\pi}{2}} e^{i \omega_{0} t}+\left(b+b_{i n t}\right) i \omega_{0} x_{0} e^{-i \frac{\pi}{2}} e^{i \omega_{0} t}+\left(k+k_{\text {int }}\right) x_{0} e^{-i \frac{\pi}{2}} e^{i \omega_{0} t}=A_{0} e^{i \omega_{0} t}
$$

Replacing $i^{2}=-1$,

$$
-m\left(\omega_{0}\right)^{2} x_{0} e^{-i \frac{\pi}{2}} e^{i \omega_{0} t}+i\left(b+b_{\text {int }}\right) \omega_{0} x_{0} e^{-i \frac{\pi}{2}} e^{i \omega_{0} t}+\left(k+k_{\text {int }}\right) x_{0} e^{-i \frac{\pi}{2}} e^{i \omega_{0} t}=A_{0} e^{i \omega_{0} t}
$$

and noting that,

$$
e^{-i \frac{\pi}{2}}=\left(\cos \left(\frac{\pi}{2}\right)+i \sin \left(-\frac{\pi}{2}\right)\right)=-i
$$

we have,

$$
i m \omega_{0}^{2} x_{0} e^{i \omega_{0} t}+\left(b+b_{\text {int }}\right) \omega_{0} x_{0} e^{i \omega_{0} t}-i\left(k+k_{\text {int }}\right) x_{0} e^{i \omega_{0} t}=F_{0} e^{i \omega_{0} t}
$$

Rearranging terms,

$$
\left(m \omega_{0}^{2} x_{0}-\left(k+k_{\text {int }}\right) x_{0}\right) i+\left(\left(b+b_{\text {int }}\right) x_{0} \omega_{0}-F_{0}\right)=0
$$

And finally, noting that for any real numbers $A, B, C, D$, if $A i+B=C i+D$ then $A=$ $C$ and $B=D$. Thus, 


$$
\begin{gathered}
\left(k+k_{\text {int }}\right)=m \omega_{0}^{2} \\
k_{\text {int }}=m \omega_{0}^{2}-k \\
k_{\text {int }}=m \omega_{0}^{2}-m \omega_{00}^{2} \\
k_{\text {int }}=m\left(\left(\Delta \omega_{0}+\omega_{00}\right)^{2}-\omega_{00}^{2}\right) \\
k_{\text {int }}=m\left(\left(\frac{\Delta \omega}{\omega_{00}}+1\right)^{2} \omega_{00}^{2}-\omega_{00}^{2}\right) \\
k_{\text {int }}=m \omega_{00}^{2}\left(\left(\frac{\Delta \omega}{\omega_{00}}+1\right)^{2}-1\right) \\
k_{\text {int }}=k\left(\left(\frac{\Delta \omega}{\omega_{00}}+1\right)^{2}-1\right)
\end{gathered}
$$

Similarly,

$$
\begin{gathered}
\left(b+b_{\text {int }}\right) x_{0} \omega_{0}=F_{0} \\
b_{\text {int }}=\frac{F_{0}}{x_{0} \omega}-b \\
b_{\text {int }}=\frac{F_{0}}{x_{0} \omega_{0}}-\frac{F_{0}}{x_{00} \omega_{00}} \\
b_{\text {int }}=F_{0}\left(\frac{1}{x_{0} \omega_{0}}-\frac{1}{x_{00} \omega_{00}}\right) \\
b_{\text {int }}=F_{0}\left(\frac{x_{00} \omega_{00}}{x_{0} \omega_{0}}-1\right)
\end{gathered}
$$




$$
b_{\text {int }}=F_{0} \frac{1}{x_{00} \omega_{00}}\left(\frac{x_{00} \omega_{00}}{x_{0} \omega_{0}}-1\right)
$$

and realizing that,

$$
\frac{k x_{00}}{Q}=F_{0}
$$

we have the final expressions used in the body of the thesis to calculate $k_{\text {int }}$ and $b_{\text {int }}$,

$$
\begin{gathered}
k_{\text {int }}=\frac{k x_{00}}{Q} \frac{1}{x_{00} \omega_{00}}\left(\frac{x_{00} \omega_{00}}{x_{0} \omega_{0}}-1\right) \\
b_{\text {int }}=\frac{k}{Q} \frac{1}{\omega_{00}}\left(\frac{x_{00} \omega_{00}}{x_{0} \omega_{0}}-1\right)
\end{gathered}
$$

where $x_{0}$ is the amplitude at resonance while interacting, $x_{00}$ is the amplitude at resonance $\omega_{00}=2 \pi f_{00}$ with $f_{00}$ the frequency at resonance before interaction, $\omega_{0}=$ $2 \pi f_{0}$ with $f_{0}$ the frequency at resonance while interacting and $\omega_{0}=\Delta \omega_{0}+\omega_{00}$. 


\section{APPENDIX B}

\section{Calculation of the piezo-electro-mechanical coupling $\alpha$}

The calculation of the QTF has been extensively analyzed in previous publications[65], [72], [73], [76], [134]. Here offer a summary of the calculations needed to electrically calibrate the QTF's amplitude of oscillation. The calculation of the QTF begins with the determination of the capacitance of the mechanical model for the crystal quartz oscillators. By fitting the measured curve to the equation,

$$
I(\omega)=\frac{I_{0} \omega}{Q \omega_{0}} \sqrt{\frac{1+2 \frac{C_{0}}{C}\left(1-\frac{\omega^{2}}{\omega_{0}^{2}}\right)+\frac{C_{0}^{2}}{C^{2}}\left(1-\frac{\omega^{2}}{\omega_{0}^{2}}\right)^{2}+\frac{C_{0}^{2}}{C^{2}}\left(\frac{\omega}{\omega_{0} Q}\right)^{2}}{\left(1-\frac{\omega^{2}}{\omega_{0}^{2}}\right)^{2}+\left(\frac{\omega}{\omega_{0} Q}\right)^{2}}}
$$

With the additional relations $\omega_{0}=1 / \sqrt{L C}, Q=L \omega_{0} / R$ and $I_{0}=V / R$ you obtain $\mathrm{R}$, then $L$ then $C$. Or by combining all three equations,

$$
C=\frac{1}{R Q \omega_{0}}
$$

where $R, Q$ and $\omega_{0}$ are results from the fitting. The stiffness $k$ is calculated from,

$$
k=\frac{E}{4}\left(\frac{t}{L}\right)^{3} w
$$

To obtain the piezo-electro-mechanical-coupling $\alpha$ we start with the relation between charge and tine displacement $Q_{\text {charge }}=\alpha x$. Since both tines are moving and they move with $180^{\circ}$ out of phase, the right expression for the two tines is, 


$$
Q_{\text {charge }}=2 \alpha x
$$

The energy of a capacitor of charge $Q_{\text {charge }}$ is equal to the kinetic energy of both tines,

$$
\begin{gathered}
\frac{Q_{\text {charge }}^{2}}{2 C}=2\left(\frac{k x^{2}}{2}\right) \\
\frac{(2 \alpha x)^{2}}{C}=2\left(k x^{2}\right) \\
\frac{(\alpha)^{2}}{C}=\frac{k}{2}
\end{gathered}
$$

and we obtain,

$$
\alpha=\sqrt{\frac{k C}{2}} .
$$

Finally the $\Delta I_{R M S} / \Delta x_{R M S}$ is found from,

$$
\Delta I_{R M S}=4 \pi f \alpha \Delta x_{R M S}
$$




\section{APPENDIX C}

\section{The problem of the changing tunneling impedance}

The bias voltage in tunneling current changes as the tunneling resistance decreases. This fact makes the actual measured I/V ratio to change.
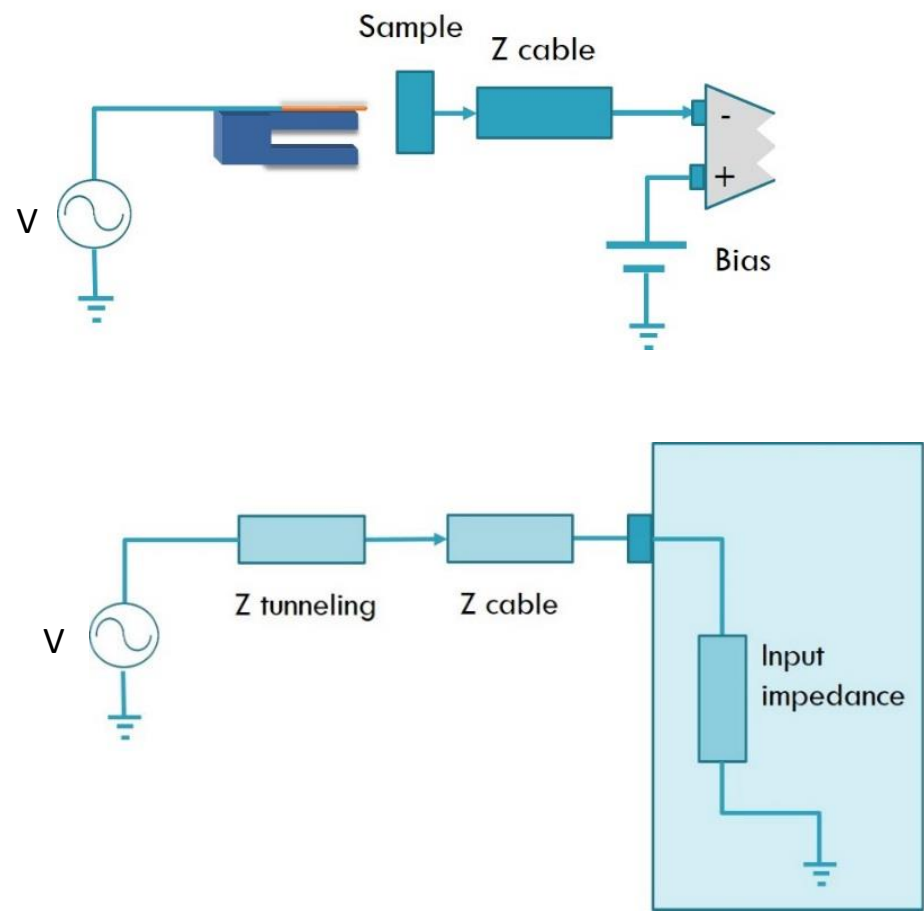

Figure C.1 Top: Connections for the tunneling current measurements. Bottom: Schematic for the analysis for the measured current logarithmic behavior. The bias is set a dc voltage and does not affect the ac measurements (it is different than zero only in the case of the approaches on silicon).

Here we present an analysis that shows how the measured current, even under this conditions, will produce a logarithmic trend. First we express the voltage across the 161 
tunneling impedance as (using Figure C.1)

$$
V_{\text {Tunneling }}=\frac{V}{Z_{\text {Tunneling }}+Z_{\text {Cable }}+Z_{\text {input impedance }}} \times\left(Z_{\text {input impedance }}+Z_{\text {Cable }}\right)
$$

where $\mathrm{V}$ is the voltage amplitude of the ac bias voltage. Next, the expression for the tunneling current $I_{\text {Tunneling }}$ is,

$$
\begin{aligned}
& I_{\text {Tunneling }}=\frac{V_{R}}{Z_{\text {Tunneling }}}=\frac{V}{Z_{\text {Tunneling }}+Z_{\text {Cable }}+Z_{\text {input impedance }}} \times \frac{\left(Z_{\text {input impedance }}+Z_{\text {Cable }}\right)}{Z_{\text {Tunneling }}} \\
& I_{\text {Tunneling }}=I_{\text {Measured }} \times \frac{\left(Z_{\text {input }}+Z_{C}\right)}{Z_{T}} \\
& \operatorname{Ln}\left(I_{\text {Tunneling }}\right)=\operatorname{Ln}\left(I_{\text {Measured }}\right)+\operatorname{Ln}\left(\frac{\left(Z_{\text {input }}+Z_{\text {Cable }}\right)}{Z_{\text {Tunneling }}}\right) \\
& \operatorname{Ln}\left(I_{\text {Tunneling }}\right)=\operatorname{Ln}(I)+\operatorname{Ln}\left(Z_{\text {input }}+Z_{\text {Cable }}\right)-\operatorname{Ln}\left(Z_{\text {Tunneling }}\right) \\
& \operatorname{Ln}\left(I_{\text {Tunneling }}\right)=\operatorname{Ln}\left(I_{\text {Measured }}\right)-2 z \sqrt{\frac{2 m \varphi}{\hbar^{2}}}+\text { Const } \\
& \operatorname{Ln}\left(I_{\text {Tunneling }}\right)=\operatorname{Ln}\left(I_{\text {Measured }}\right)-C 1 z+C 2
\end{aligned}
$$

Using equation ( 17 ) for the $\boldsymbol{I}_{\text {Tunneling }}$ shows that the logarithm of the $\boldsymbol{I}_{\text {Measured }}$ is proportional to the probe-sample distance plus a linear term and a constant. As a consequence, the current measured with the circuit shown in Figure C.1 is expected to behave linearly in a logarithmic scale. 


\section{APPENDIX D}

\section{Difference in the force analysis between a QTF and a cantilever type probes}

The difference in the calculation of the interaction forces for the cantilever and the QTF is shown in (Figure D.1). To calculate the average oscillation of the cantilever probe, assuming a peak to peak oscillation spanning three positions $\left(z_{1}, z_{2}\right.$ and $\left.z_{3}\right)$ the average interaction for the force in the position $z_{3}$ is $A\left(z_{3}\right)=\left(A\left(z_{1}\right)+A\left(z_{2}\right)+A\left(z_{3}\right)\right) / 3$. As seen in Figure D.2 the results can be generalized to $A\left(z_{n}\right)$. The main characteristic is that every average position depends on the result of the next. Therefore, it is necessary to arrange the results in matrix form and solve the system of equations. This is the essence of the Giessibl method. Sader used an integral form to attack the same problem.

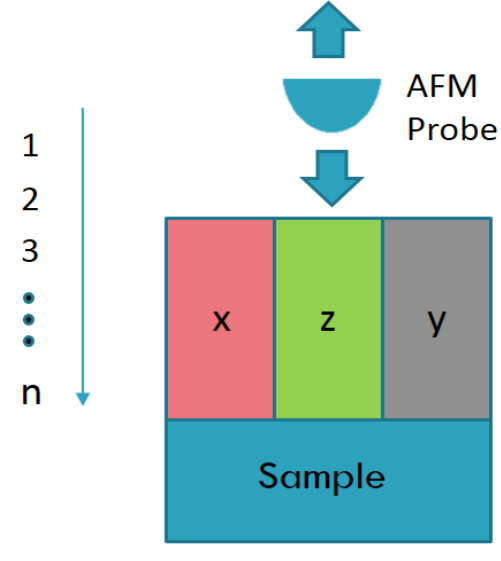

Cantilever

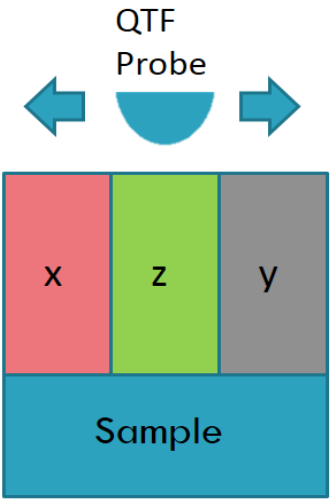

Quartz Tuning Fork

Figure D.1 Diagram showing the difference in the calculation of the average force for a cantilever and the quartz tuning fork. The cantilever interacts along the $Z$ region. The $Q T F$ interacts with the $X, Y$ and $Z$ regions. 
Position $1: \frac{A\left(z_{1}\right)+A\left(z_{2}\right)}{2}$

Position $2: \frac{A\left(z_{1}\right)+A\left(z_{2}\right)+A\left(z_{3}\right)}{3}$

Position $3: \frac{A\left(z_{2}\right)+A\left(z_{3}\right)+A\left(z_{4}\right)}{3}$

AFM Cantilever Probe

$\mathrm{z}_{1}$

$\mathrm{z}_{2}$

$\mathrm{z}_{3}$

$\mathrm{z}_{4}$

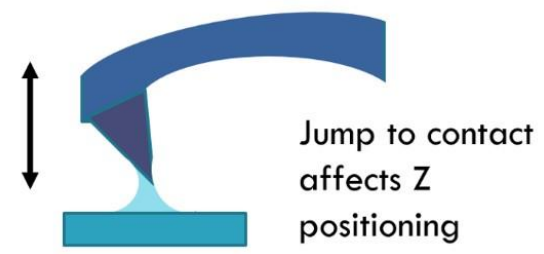

Position $\mathrm{n}: \frac{A\left(z_{n, 1}\right)+A\left(z_{n}\right)+A\left(z_{4 n, 1}\right)}{3}$

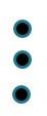

$z_{n}$

Figure D.2 The matrix method to solve the force interactions of the AFM cantilever probe in frequency modulation mode.

Position 1 $: \frac{A\left(x_{0}\right)+A\left(z_{1}\right)+A\left(y_{2}\right)}{2}$
Position $2: \frac{A\left(x_{1}\right)+A\left(z_{2}\right)+A\left(y_{3}\right)}{3}$
Position $3: \frac{A\left(x_{2}\right)+A\left(z_{3}\right)+A\left(y_{4}\right)}{3}$

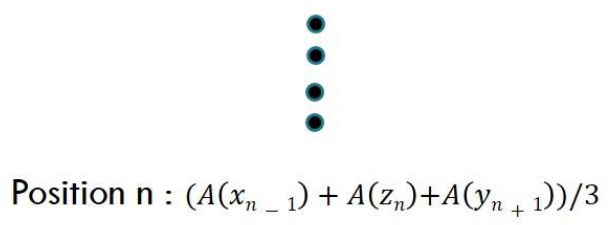

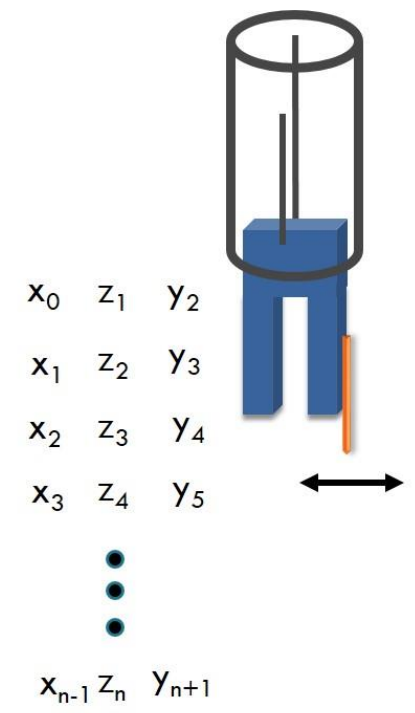

Figure D.3 Similar procedure seen in Figure D.2 but applied to the QTF. It can be seen that the force average for any position does not depend on any previous or future $z$ positions and as a consequence the average force can be entirely calculated in one cycle of oscillation. 